
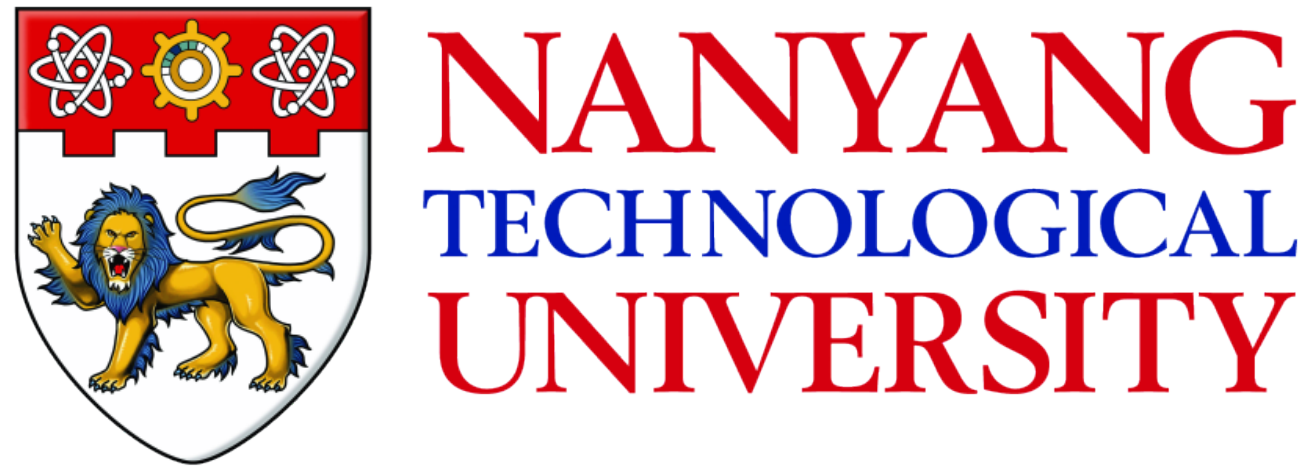

Targeted Delivery of Bio-active Molecules to Mitochondria using Mesoporous Silica Nanoparticles for Anti-cancer Applications

Candidate: Qu Qiuyu

Supervisor: Associate Prof. Zhao Yanli

School of Physical and Mathematical Sciences

A thesis submitted to the Nanyang Technological University in partial fulfilment of the requirement for the degree of Doctor of Philosophy 


\section{Targeted Delivery of Bio-active Molecules to Mitochondria using Mesoporous Silica Nanoparticles for Anti-cancer Applications}

Candidate: Qu Qiuyu

Supervisor: Associate Prof. Zhao Yanli

School of Physical and Mathematical Sciences

A thesis submitted to the Nanyang Technological University in partial fulfilment of the requirement for the degree of Doctor of Philosophy 


\section{Acknowledgements}

Firstly of all, I wish to express my sincerest acknowledgement to my supervisor Associate Professor Zhao Yanli in respect to his invaluable guidance and support. Under his supervision, I have developed systematic research skills and learnt essential knowledge under his effective academic instructions. In addition, he gave me invaluable encouragement and advice when I was confused in my research, which built my confidence and thus allowed me to complete my research.

What's more,I'd like to express my deep appreciations to the lab colleagues in Dr. Zhao's group for their helpful suggestions, discussions as well as assistance during my graduate study. I really enjoyed my research life when working and collaborating with them.

In addition, I would express special gratitude to my family for their understanding and encouragement during the period of my study abroad. Without their consistent support, I would not have been abroad for my four years' study and realized my academic dream.

Last but not least, I'd like to convey my deepest gratitude to School of Physical and Mathematical Sciences for giving me the research scholarship for pursuing my Ph.D. degree and providing the facilities and services for my research work. It is a great privilege for me to study in Nanyang Technological University, which will make meproud all my life. 


\section{Table of Contents}

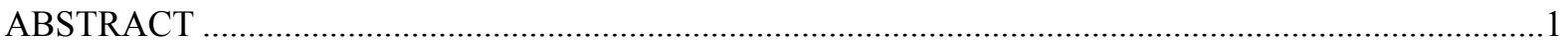

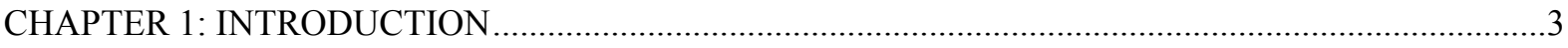

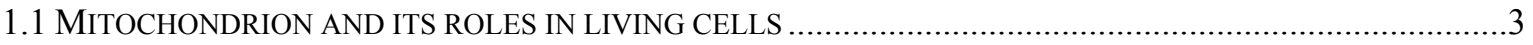

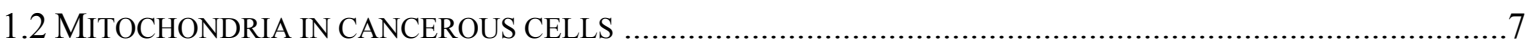

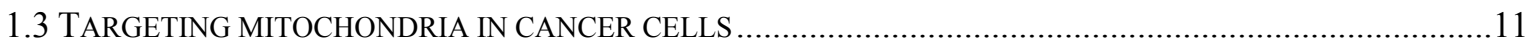

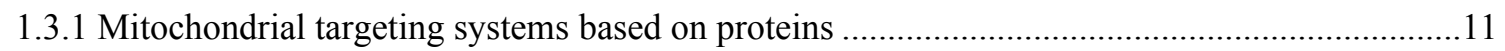

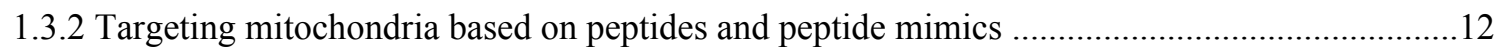

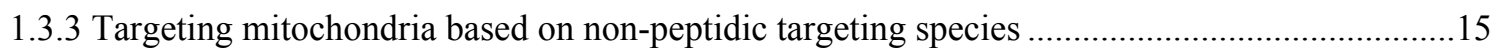

1.4 MESOPOROUS SILICA NANOPARTICLES (MSNPS): DEVELOPMENT AND APPLICATION .........................19

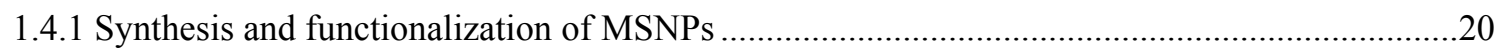

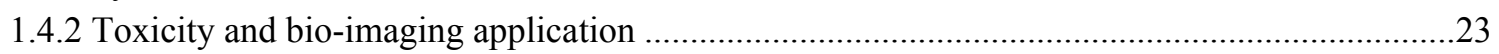

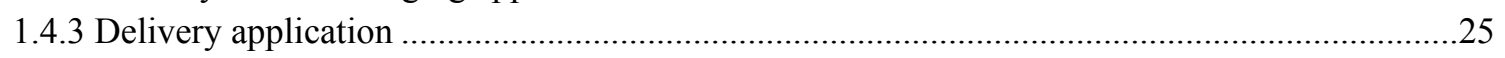

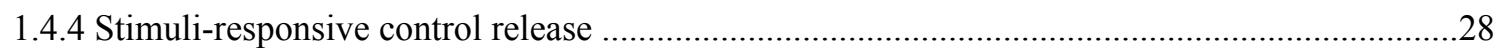

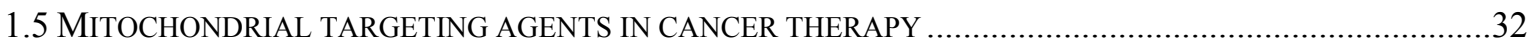

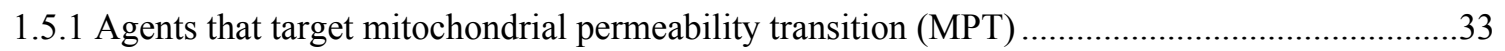

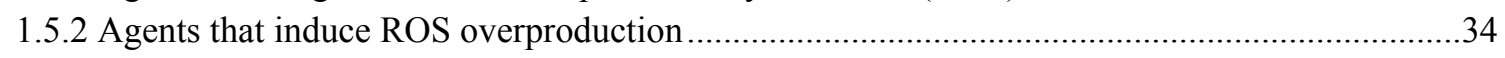

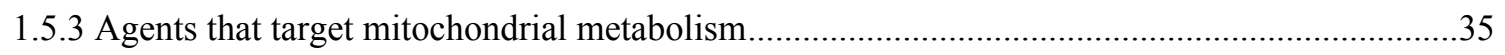

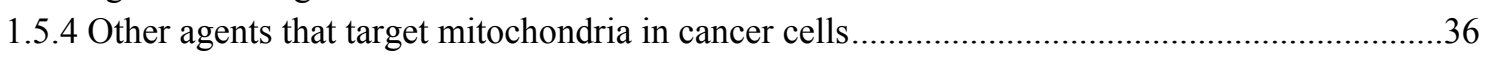

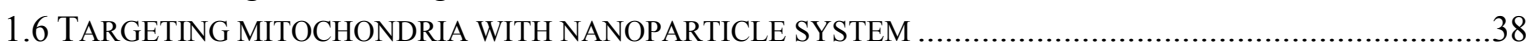

CHAPTER 2: TARGETED DELIVERY OF DOXORUBICIN TO MITOCHONDRIA USING

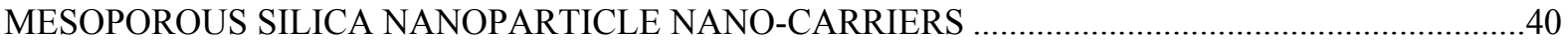

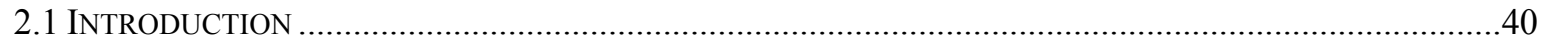

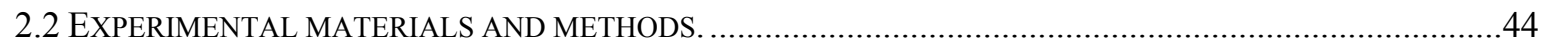

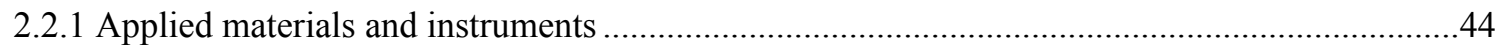

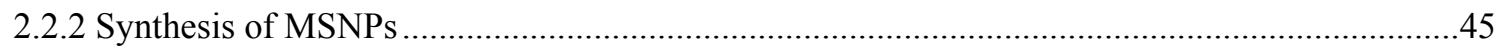

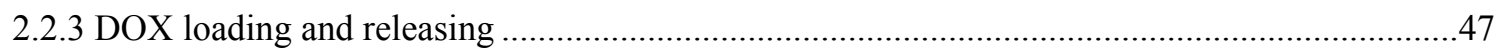

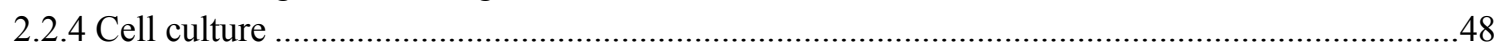

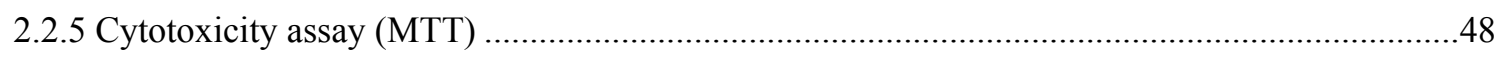

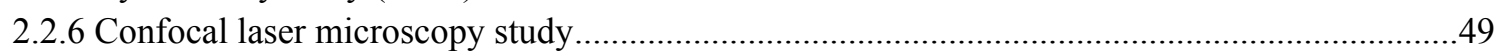

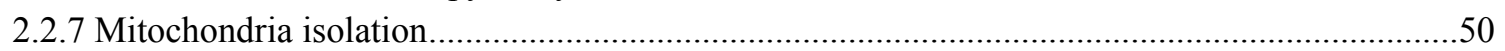

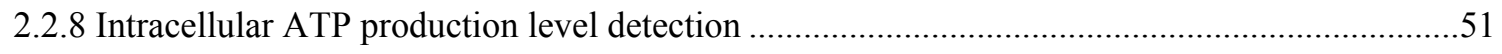

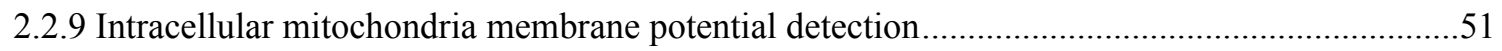

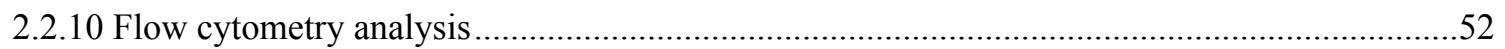

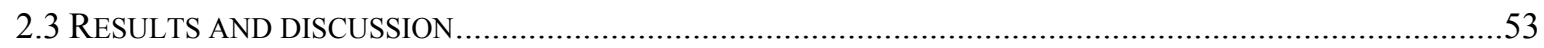

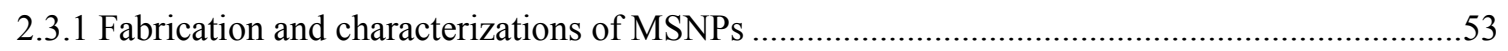

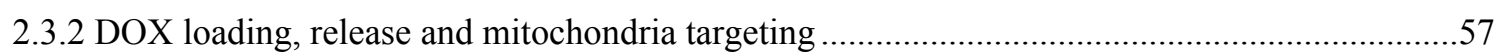

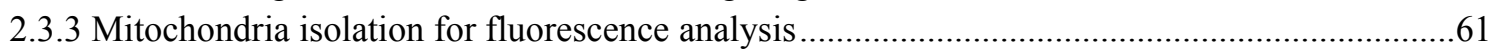

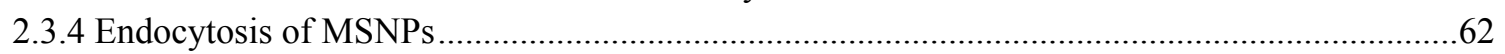

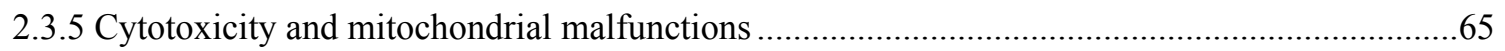

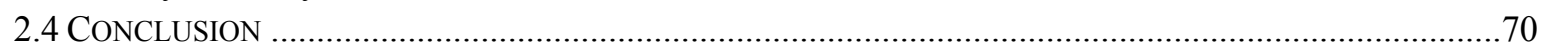

CHAPTER 3: ANTICANCER EFFECT OF A-TOCOPHERYL SUCCINATE DELIVERED BY MITOCHONDRIA-TARGETED MESOPOROUS SILICA NANOPARTICLES ……………..................71

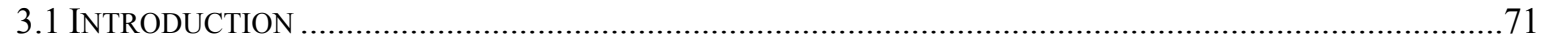

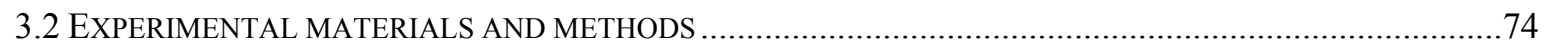

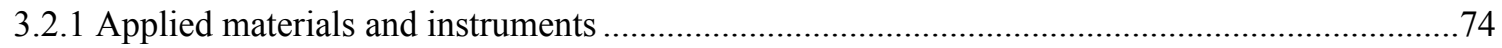




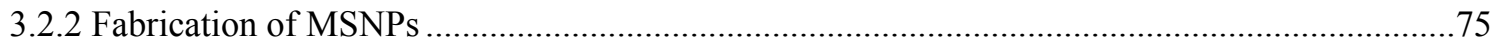

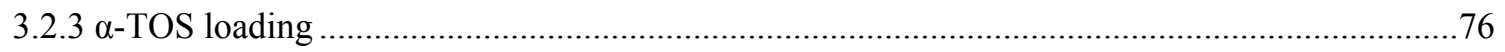

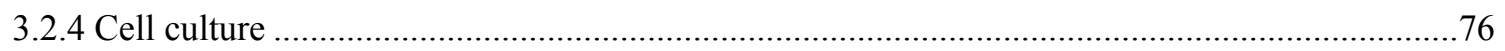

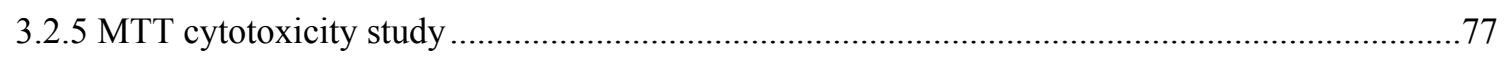

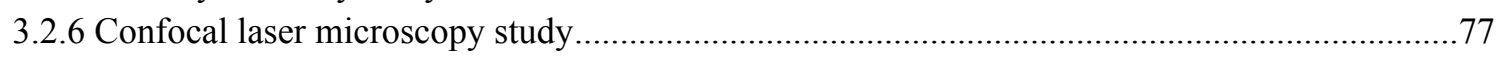

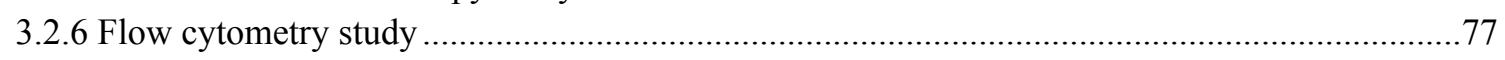

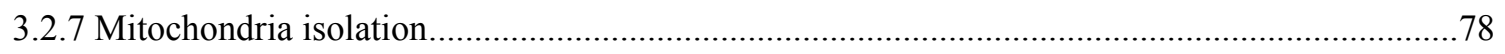

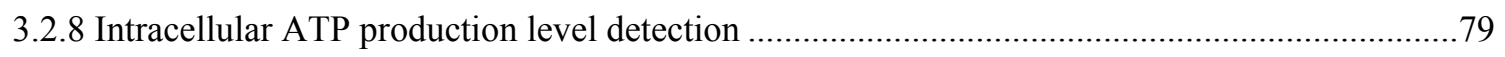

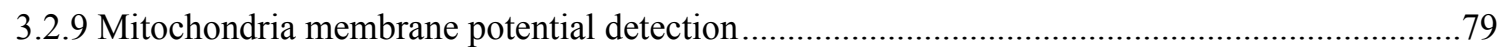

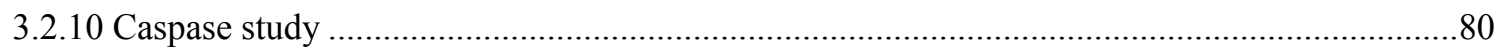

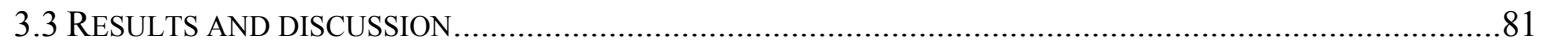

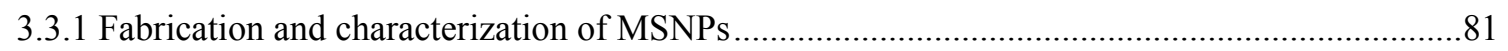

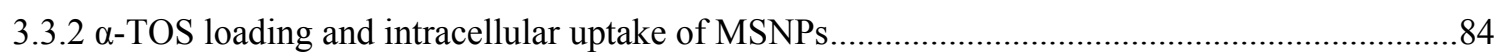

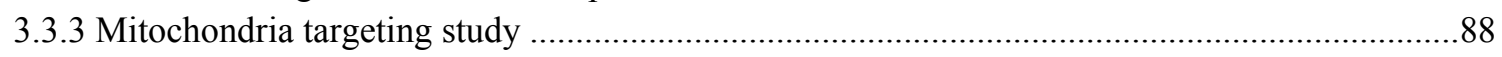

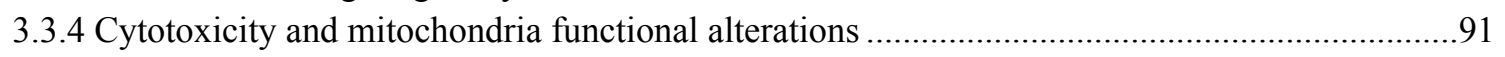

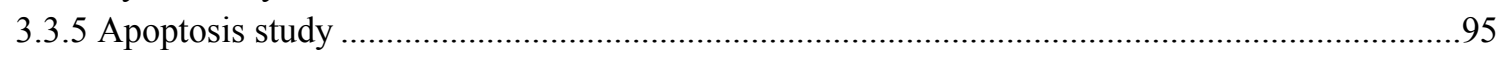

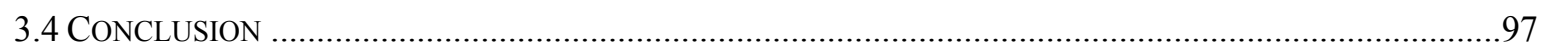

CHAPTER 4: TARGETED DELIVERY OF DOXORUBICIN TO MITOCHONDRIA BY USING MESOPOROUS SILICA NANOPARTICLES FOR OVERCOMING DRUG RESISTANCE …..............97

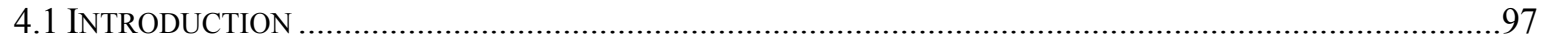

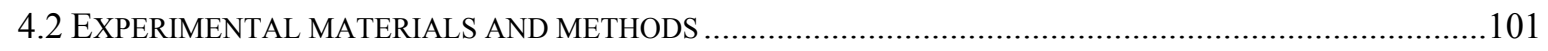

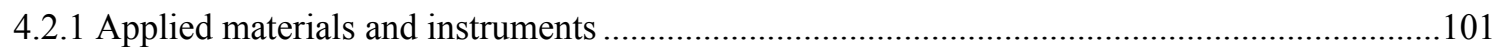

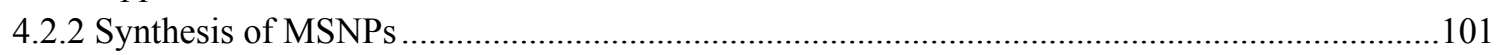

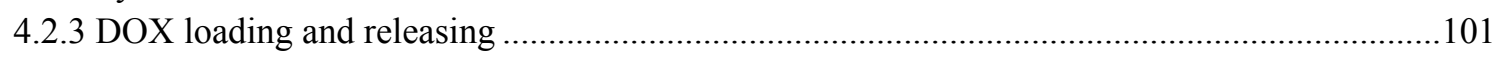

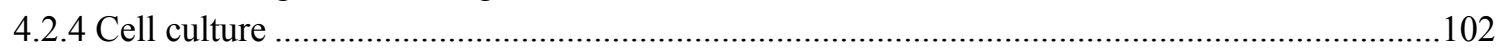

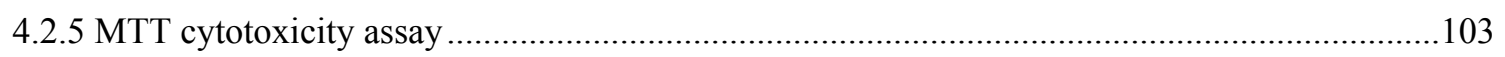

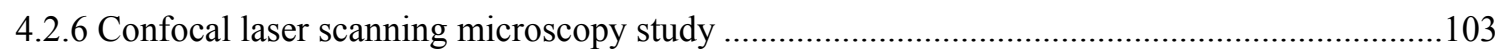

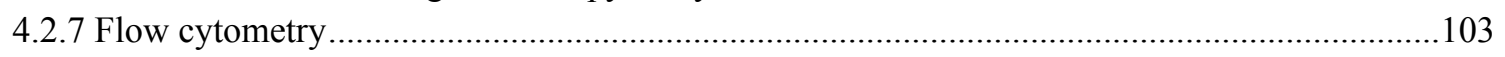

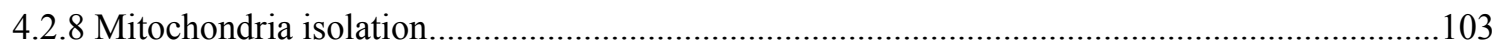

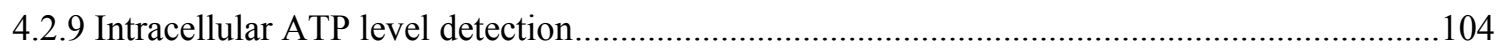

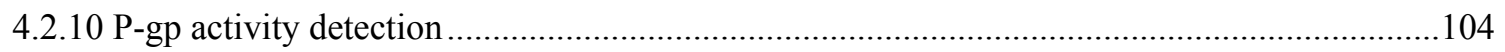

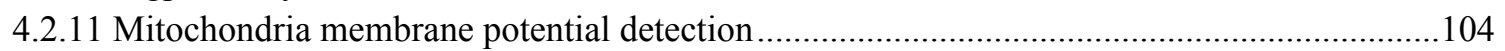

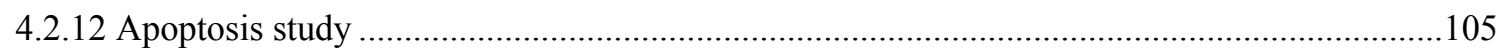

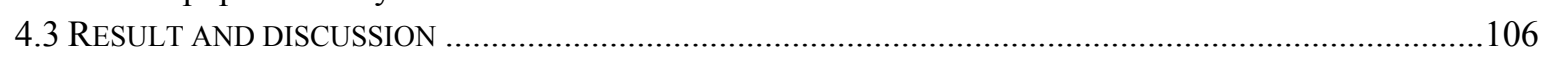

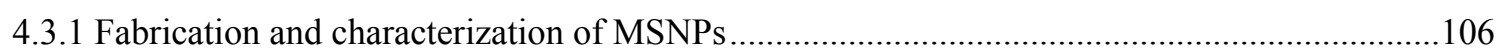

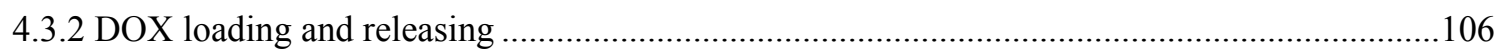

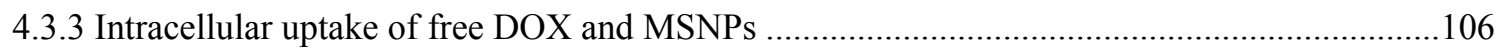

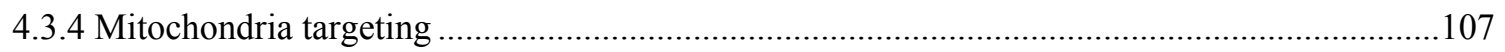

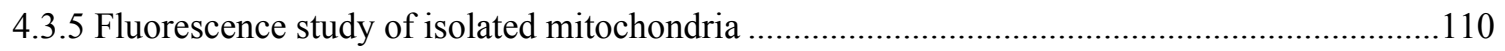

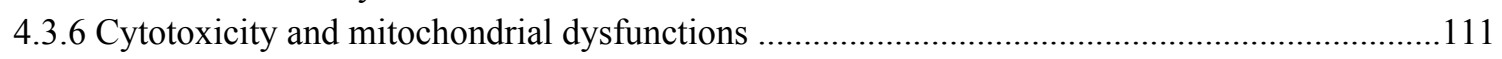

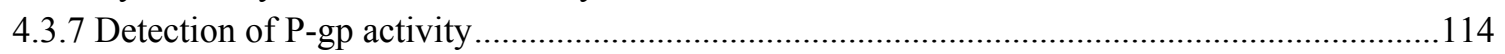

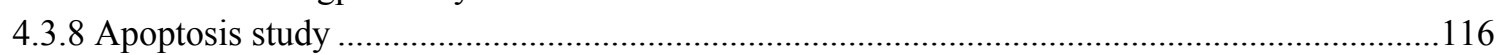

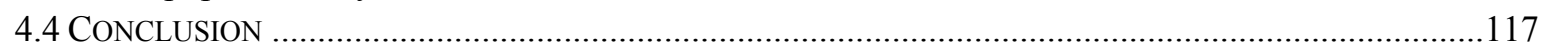

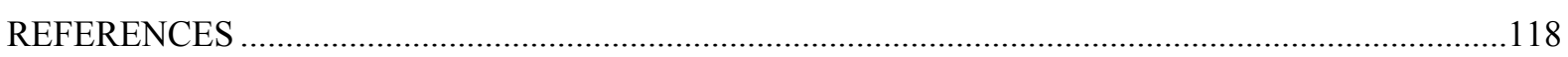

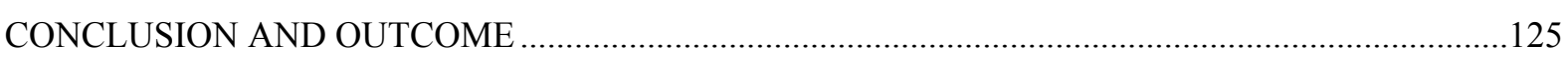

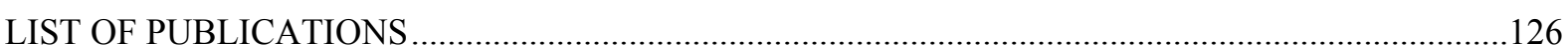




\begin{abstract}
The biological applications of mesoporous silica nanoparticles (MSNPs) in drug delivery and bio-imaging applications have been developing for decades. The continuous developments in this field are achieved by virtue of the attractive properties of MSNPs such as large surface area and volume, tunable particle and pore size and easy surface functionalization. However, the conventional MSNPs based drug delivery systems are mainly focusing on delivering drug molecules to target cells. More specific delivery applications such as sub-cellular delivery based on MSNPs have not been fully investigated and developed. In choosing the target of sub-cellular delivery of MSNPs, mitochondria appear to be a good candidate. As an important organelle in almost all living cells, mitochondria have many features that make them the possible and attractive target for cancer treatment. Such features involve energy production, cellularmetabolism and apoptotic signal transduction. In view of that, my primary research focus during Ph.D study is design, fabrication and characterization of MSNP-based system in targeted delivery of anticancer drugs to mitochondria for anticancer study as well as overcoming drug resistance.

In Chapter 2, in order to validate the application of MSNPs in sub-cellular delivery, we fabricated and characterized the obtained well-ordered MSNPs. By conjugating mitochondria targeting ligand, mitochondria targeted MSNPs were obtained and characterized. Doxorubicin (DOX) was loaded into MSNPs and the mitochondria targeting property was demonstrated by the co-localization study of mitochondria and fluorescent MSNPs or DOX loaded MSNPs. In addition, the anticancer effect of DOX loaded MSNPs was further investigated.
\end{abstract}

After proving that the availability of MSNPs in sub-cellular drug delivery, we further chose one mitochondria specific hydrophobic anticancer drug, $\alpha$-Tocopheryl Succinate $(\alpha-$ TOS) as payload to be delivered by MSNPs with reduced particle size. In Chapter 3, we 
applied $\alpha$-TOS as effective mitochondria specific anticancer drug and its hydrophobicity drawback could be overcome by MSNPs. Thus, the anticancer effectiveness of $\alpha$-TOS was maximized by MSNPs after targeted delivery to mitochondria. The maximized anticancer effectiveness of $\alpha$-TOS was demonstrated by cytotoxicity study. In addition, the $\alpha$-TOS loaded MSNPs with mitochondria targeting property also showed effectiveness in inducing programmed cell death, further validating the application potential of MSNPs in sub-cellular drug delivery.

In order to broaden the biological application of mitochondria targeted MSNPs system, their application in overcoming drug resistance was further investigated. In Chapter 4, we aim to change the target site of DOX molecules intracellularly from nucleus DNAs to mitochondria DNAs (mtDNAs) to overcome DOX resistance. After targeted delivery of DOX molecule to mitochondria, such delivered DOX molecules were found to be able to induce the decrease in ATP supply and depletion of mitochondrial membrane potential, leading to the dysfunction of P-glycoprotein (P-gp) on cell membrane which is responsible for DOX efflux, and the induction of apoptosis.

As such, the current research may be helpful in providing new insight in developing MSNPs in sub-cellular drug delivery and their applications in anticancer treatment including overcoming drug resistance. 


\section{Chapter 1: Introduction}

\subsection{Mitochondrion and its roles in living cells}

In most eukaryotic cells, there are many organelles to support the normal functions of living cells including nucleus, ribosome, rough and smooth endoplasmic reticulum (ER), golgi apparatus, cytoskeleton, mitochondrion, cytosol, lysosome and cell membrane. Among these important organelles, mitochondrion is universally exist in all eukaryotic organismsexcept Monocercomonoides, which is the only one eukaryote known to lost the mitochondrion completely, although some cells may lack mitochondrion under particular circumstances like red blood cells. Thus, from the view of evolution, mitochondrion is always having its roles and functions along the way of evolution of eukaryotic organisms ${ }^{1-4}$.

Generally, a typical mitochondrion is consist of five major parts which are the outer mitochondrial membrane, the inner mitochondrial membrane, the inter-membrane space, the cristae space formed by infoldings of inner membrane and the matrix within the inner membrane (Figure 1). The outer mitochondrial membrane encloses the entire space within it and it contains many proteins that similar to cell membrane. Among those proteins, porin is a type of protein that can form channels to allow small molecule (less than 5000 daltons) to diffuse across the membrane ${ }^{5}$. In addition, the outer membrane of mitochondrion is associated with other organelles like ER through a structure called mitochondria-associated ER membrane. Unlike mitochondrial outer membrane, the inner membrane is highly impermeable to molecules since it does not contain porin protein. Therefore, the inner membrane contains a large number of proteins called membrane transporters with the function to facilitate the entrance and exit of molecules. More importantly, the inner membrane is the main location for ATP synthesis including electron transport. Also, the inner membrane exhibits membrane potential, resulting in the negative charge in matrix ${ }^{6}$. The inter- 
membrane space is the space between outer and inner membrane. Within such space, the concentrations of small molecules are the same as in the cytosol since the outer membrane is highly permeable. Matrix is the space inside inner membrane which contains highly concentrated enzyme mixture that facilitates the ATP synthesis and some other major functions like oxidation of fatty acids. It is also the place that contains mitochondrial genetic materials including RNAs and mitochondrial DNAs ${ }^{7-10}$.
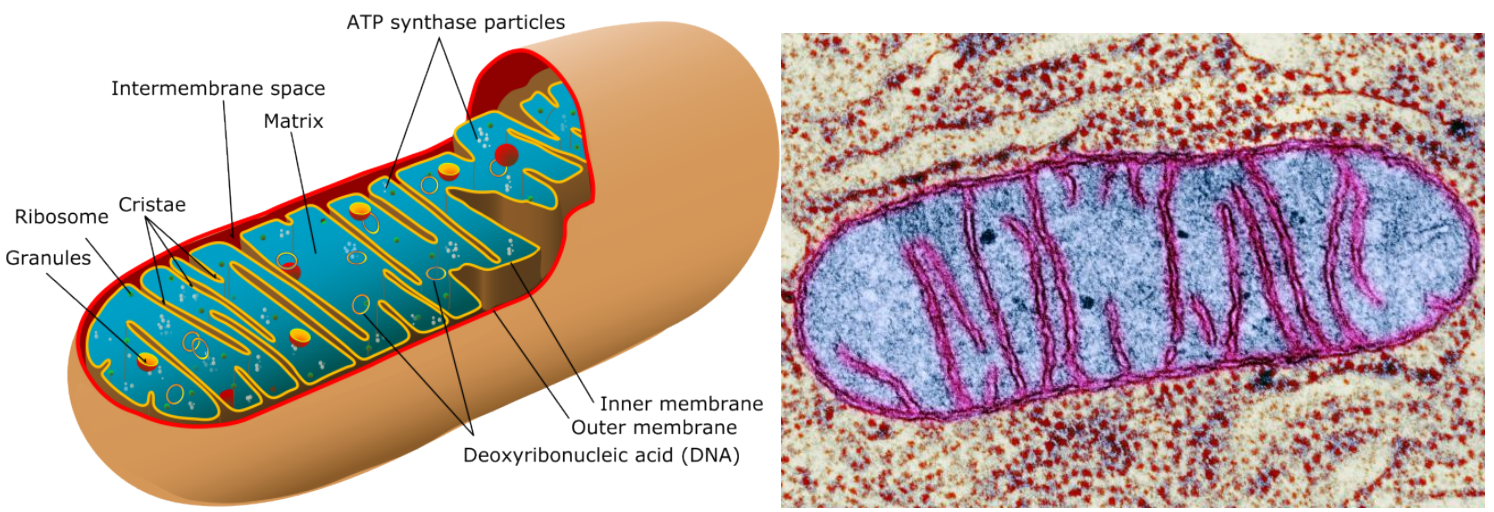

Figure 1.Typical structure of a mitochondrion (left) and colored transmission electron microscope (TEM) image of a mitochondrion (right).(Reproduced from https://simple.wikipedia.org/wiki/Mitochondria.)

There are many important features that make mitochondrion crucial in supporting the functions of organismsthroughout the evolution. Firstly, the most dominant feature of mitochondrion is its role in energy production (Figure 2). Mitochondrion is the main place for generating adenosine triphosphate (ATP) wh0ich is the most common energy form in living organisms ${ }^{11-16}$. Such energy production process mainly occurs in the inner membrane. It is generally accepted that there are two major types of cellular respiration ${ }^{17-21}$. The first type is called aerobic respiration, which happens when in the presence of oxygen. The normal substrate of such respiration reaction is pyruvate and $\mathrm{NADH}$, the major products of glucose. The respiration reactions with oxygen have high yield of ATP molecules ${ }^{22-25}$. However, when oxygen is limited, the respiration reactions will divert to the other type called anaerobic metabolism, which has lower yield of ATP production compared to aerobic respiration ${ }^{26-28}$. Approximately, the ATP production yield of aerobic respiration is 13 times higher than that 
in anaerobic respiration. The ATP energy production feature of mitochondrion lays the foundation of all normal functions of living organisms and the metabolic reactions occurring in mitochondrion provide various substrates for construction of other cellular structures ${ }^{29-32}$.

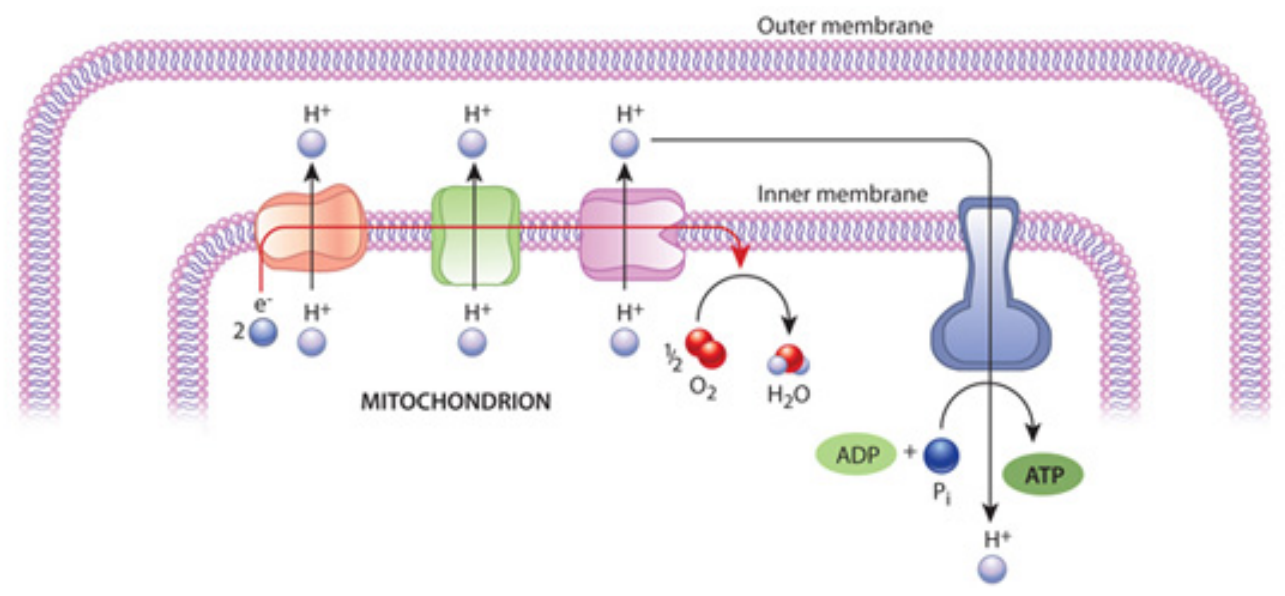

Figure 2.Illustration of the ATP synthesis and electrochemical proton gradient in mitochondria.(Reproduced with permission from reference ${ }^{9}$.)

Secondly, mitochondrion is the major intracellular place for the storage of calcium ions ${ }^{33-36}$. The contribution of mitochondrion in storing calcium ions maintains the intracellular homeostasis of calcium ions. In fact, mitochondrion has the ability to quickly uptake calcium ions for later release, buffering the concentration of calcium ions in cytosol. In addition, it is noted that the concentration of free calcium ions in cell is closely related with the regulation of various reactions whichare important for signal transductions within cells. Driven by the mitochondrial membrane potential, free calcium ions can be uptaken by mitochondrion to matrix. However, the release of stored calcium ions back into cytosol can induce and activate a series of second messenger proteins, further resulting in regulations of biological process such as the release of hormones ${ }^{37-39}$.

Thirdly, mitochondrion plays central role in regulation of programmed cell death: apoptosis (Figure 3$)^{7}$, 40-44. Apoptosis occurs in multicellular living organisms, leading to cell morphology change and further cell death. It is estimated that every day, around 50 to 70 billion cells die from apoptosis in human adult. The most characteristic feature of apoptosis is 
that it is a highly regulated process $17,45-48$. Based on current research, it is generally believed that there are two ways to initiate apoptosis: intrinsic pathway and extrinsic pathway ${ }^{49-54}$. Each pathway of apoptosis contains the recognition of apoptotic signal that inform cells to initiate the apoptosis process. In intrinsic pathway, the apoptotic signal is recognized when cells feel stress and decide to kill themselves ${ }^{55}$. While in extrinsic pathway (Figure 3), cells kill themselves when apoptotic signal is received, which is originated from other cells. For both pathways, apoptosis will be activated when apoptotic signalsare detected by cells. Mitochondrion plays an essential role in intrinsic apoptosis pathway ${ }^{56-58}$. Pro-apoptotic signals or protein molecules will affect the functions of mitochondrion in different ways. These may include the formation of mitochondrial membrane pores, which will increase the permeability. Such pores will release apoptotic effectors that will be able to initiate apoptosis process. In other words, pro-apoptotic signals will inform mitochondrion to begin the apoptosis by releasing apoptotic effectors. Specifically, an important apoptotic effector called cytochrome c, which plays an important role in mitochondrial respiratory chain, will be released from the outer mitochondrial membrane due to the formation of mitochondria apoptosis-induced channel (MAC) $)^{59}$. Once the release of cytochrome c, such molecules will combine with subsequent apoptotic factors to activate apoptosis. Therefore, as mentioned, mitochondrion is the central control point in apoptosis activation process. Last but not least, mitochondrion is the main place for the production of reactive oxygen species (ROS) which is responsible for cellular oxidative stress and aging ${ }^{60-63}$. In addition, mitochondrion is the central control point in many cellular activities such as the regulation of membrane potential, cellular metabolism and hormonal signaling ${ }^{64-66}$. 


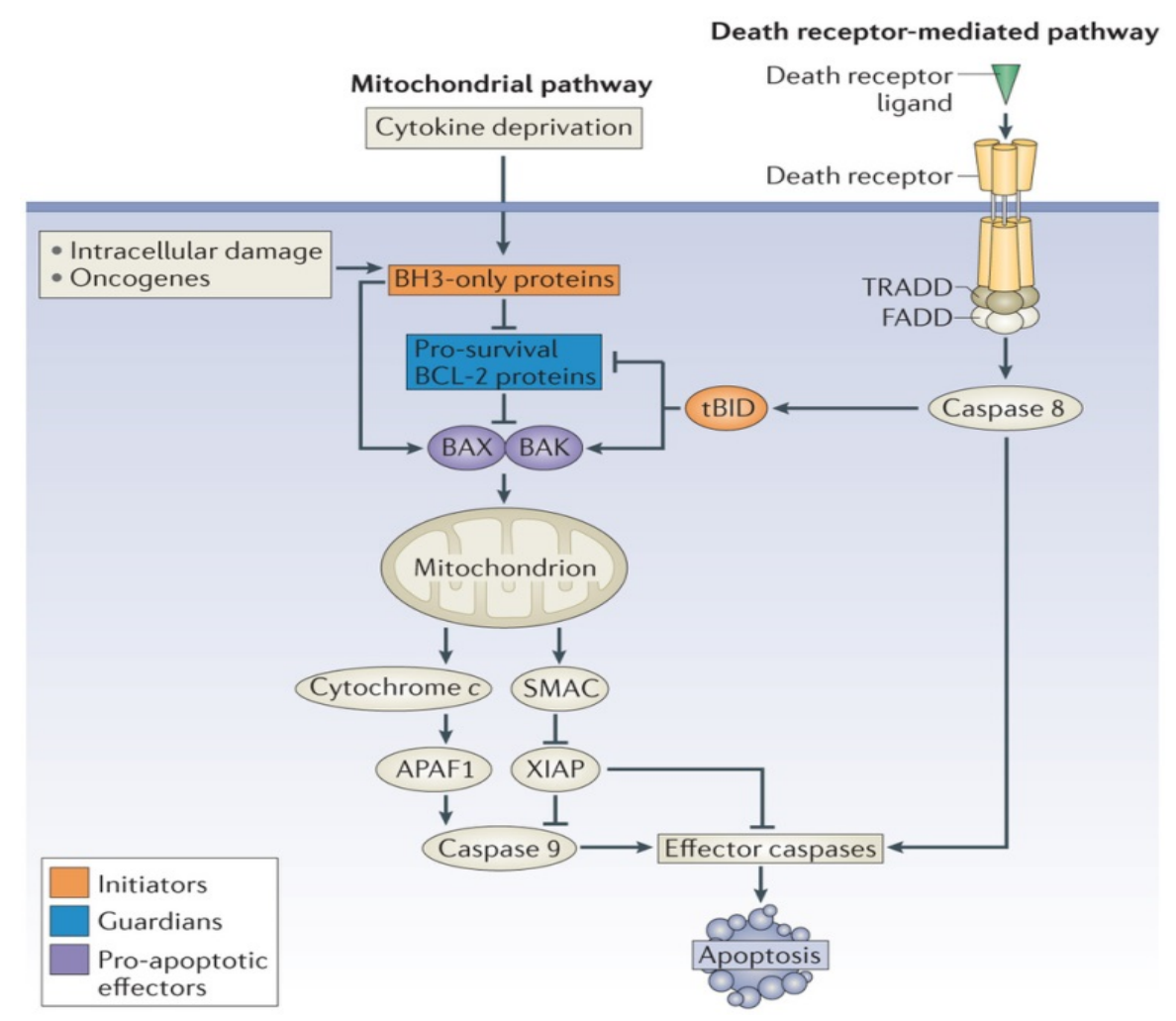

Figure 3. The apoptosis pathways mediated by mitochondria and various cell death initiators and receptors.(Reproduced with permission from reference ${ }^{55}$, Copyright 2013Rights Managed by Nature Publishing Group.)

\subsection{Mitochondria in cancerous cells}

One of the major hallmarks of cancer is that cancer cells can divide continuously ${ }^{67}$. Cell division is a normal biological process in most eukaryotic cells that mother cell divides into two or more daughter cells. Such proliferation process is critical for the formation of new tissues as well as the replacement of old or died cells as a result of damage or aging. In healthy cells, the number of cell division will decrease as cell ages. However, cancer cells will continue to divide and cell-aging process is not obvious. It is known that cell division requires ATP energy supply, which is mainly produced in mitochondria. Therefore, it is necessary to discuss the relationship between mitochondria and cancer cell division.

Historically, in 1861, Louis Pasteur first discovered that yeast tended to divide more when oxygen was providedwithout limitation, while the fermentation process was decreased under the same condition. Such discovery was summarized as "Pasteur effect", indicating the 
importance of oxygen in cell division. The role of mitochondria in tumorigenesis process was not noticed and emphasized until the discovery of "Warburg effect" (Figure 4) 68-72. In 1956, biochemist Otto Warburg observed that energy is predominantly produced by way of high rate of glycolysis, a process that has low rate of energy production, rather than low rate of glycolysis and the high rate of energy production in normal cells ${ }^{73,74}$. Warburg also suggested that the change in metabolism might be the cause of cancer. According to Warburg, unlike normal cells, the place for energy production in cancer cells is not mainly in mitochondria.It is through glycolysis in cytosol ${ }^{75-77}$. It is reasonable to think that mitochondria damage in cancer cells might be responsible for the cause of Warburg effect. Considering that mitochondrion is the central point in apoptosis control, the functions of mitochondria in cancer cells become disabled otherwise apoptosis signal stemming from the formation of mitochondrial outer membrane permeability (MOMP), might be activated resulting in cancer cell death. Beyond that, Warburg effect may be associated with continuous cell division cancer cells because glycolysis process is capable to produce substrates that are the building blocks for continuous cell division ${ }^{78-83}$. Thus, Warburg effect is an important feature of most cancer cells with the possibility that mitochondrial functions in cancer cell might be compromised in terms of energy production, much more attention was paid to the roles of mitochondrion in proliferation and evolution of cancer cells. 

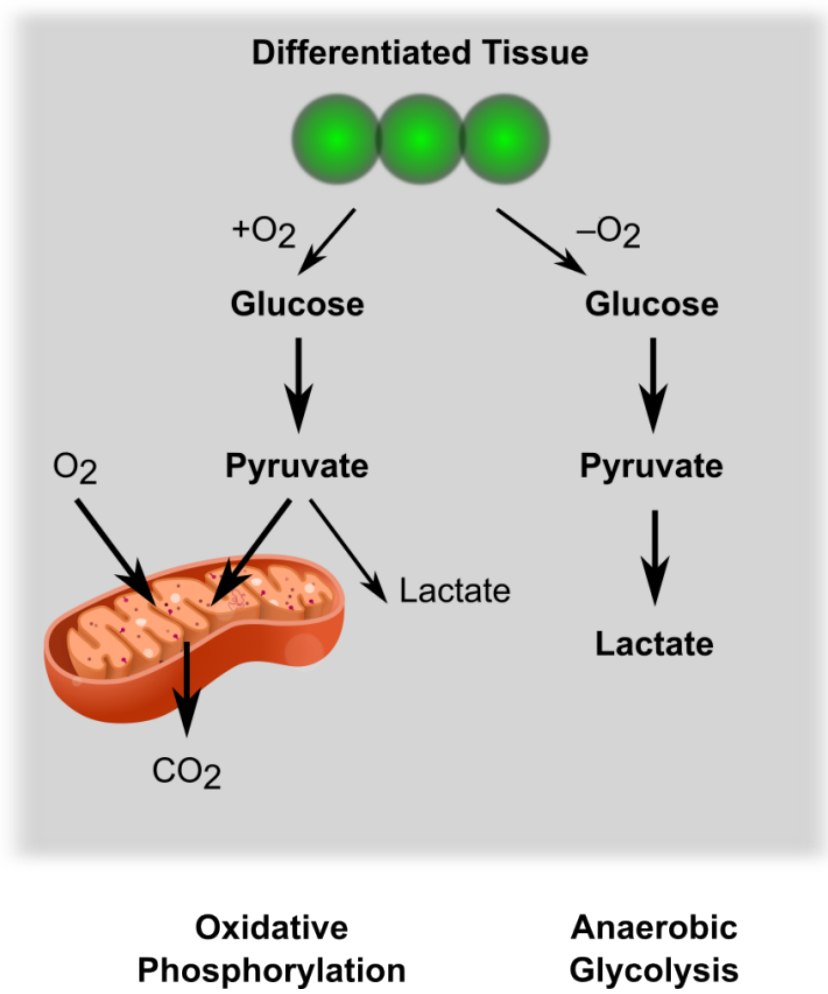

Anaerobic Glycolysis

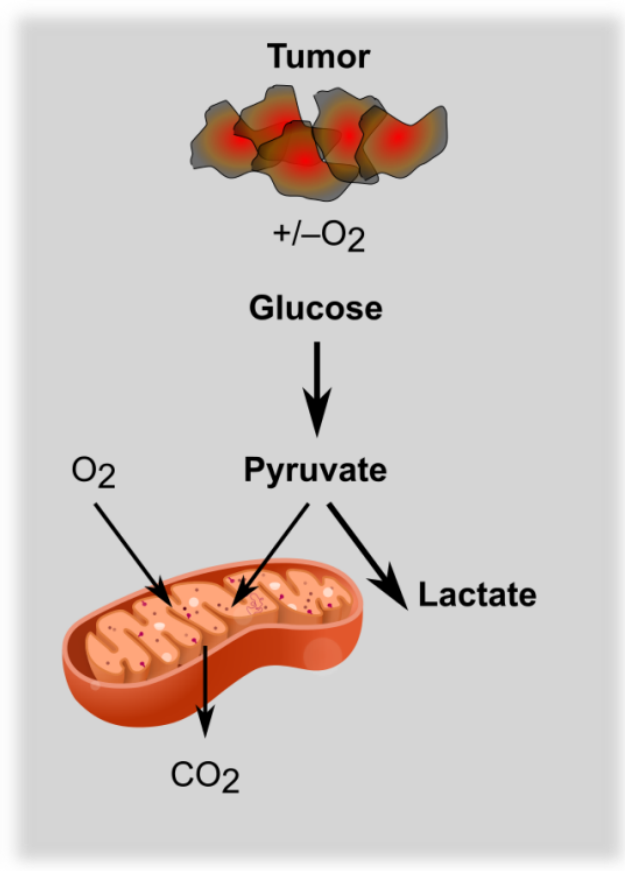

Aerobic Glycolysis (Warburg Effect)

Figure 4.Illustration of Warburg effect: the different metabolic pathways of glucose in normal and tumor tissues.(Reproduced with permission from reference ${ }^{71}$, Copyright 2009 American Association for the Advancement of Science.)

Up to date, after decades of research, it is shown that Warburg effect driven by damaged mitochondria has been demonstrated in most cases of cancer cells, while some types of cancer cells with Warburg metabolism possess normal functions of mitochondria such as mitochondrial respiration reactions ${ }^{71,76,84-86}$. However, it has been increasingly recognized thatthe functions of mitochondrion in tumorigenesis and evolution process become more and more important. For instance,functions of mitochondrion as stress sensors enable cells to adapt to various environments, and mitochondria in cancer cells also impart significant flexibilities for cancer cells to survive in different environments such as hypoxia and cancer treatment.

As discussed above, mitochondrion is the key player in cancer cell growth, survival and adaptation and mitochondrial functions are partially inhibited in most cases. Because of the importance of mitochondrion in cancer cells, it is not surprising that researchers consider 
mitochondrion as therapeutic target in cancer treatment ${ }^{37,87-93}$. Such consideration is based on current research on the expanding roles of mitochondrion in cancer. For example, the respiration chain can be affected by mutations in mitochondrial or nuclear DNA, resulting in the lower production of ATP energy molecule, over production of ROS and higher oxidative stress that may cause damage to other macromolecules like proteins (Figure 5). In addition, some mitochondrial DNA mutations are closely correlated with the development of various cancers such as breast and prostate cancer at an increased risk ${ }^{61,94,95}$. Furthermore, it is found that other than the fact that mitochondria in cancer cells are functionally and structurally different from their counterparts in normal cells, cancer cells exhibit high level of reprogramming in metabolism, which makes mitochondria in cancer cells more susceptible to mitochondrial dysfunctions than those in normal cells. Therefore, mitochondria in cancer cells have been developed as a promising target sites for cancer treatment ${ }^{16,96-99}$.

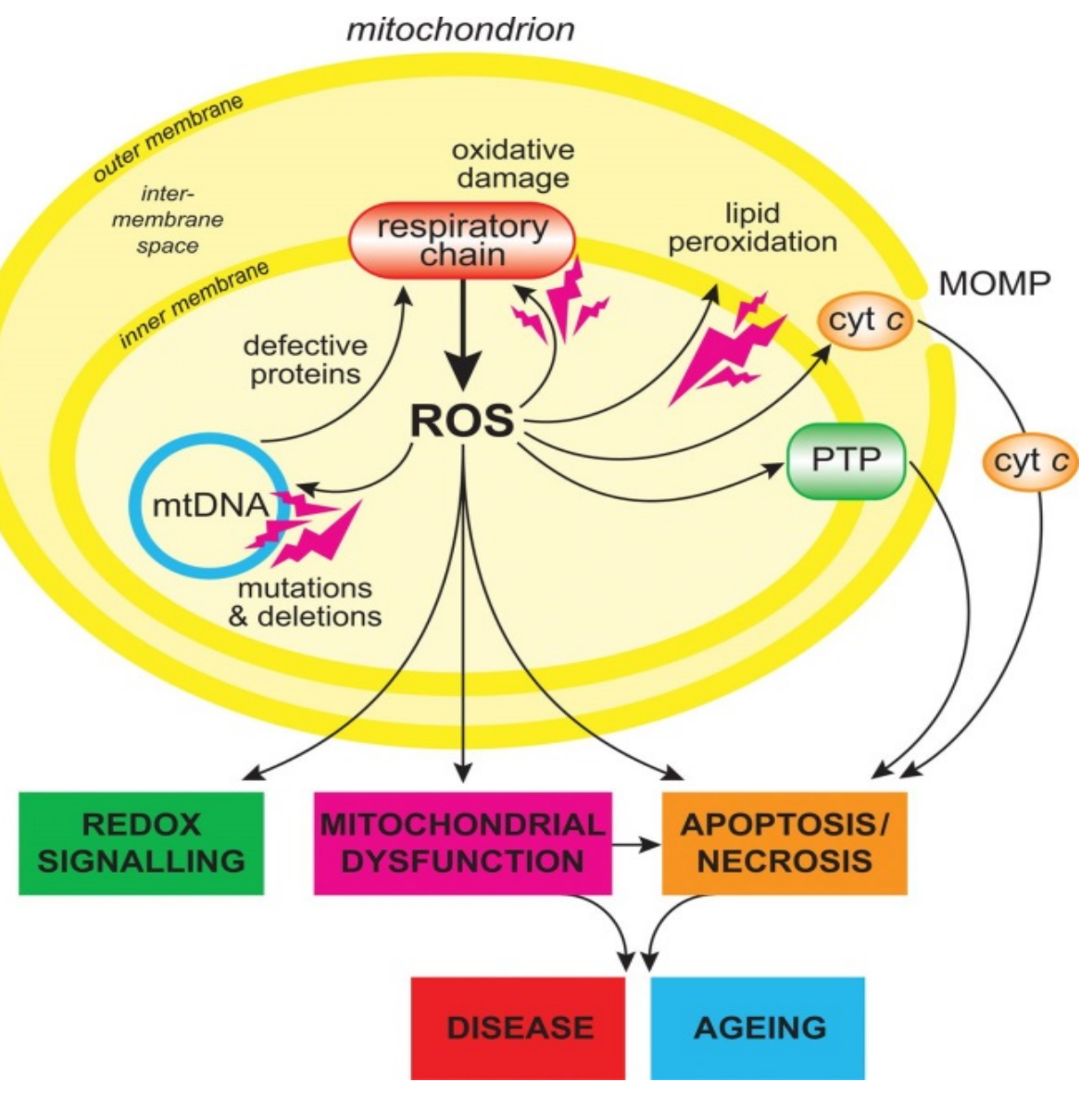


Figure 5.Overview of ROS generation in mitochondria and its resulted consequences in mitochondrial dysfunction and apoptosis.(Reproduced with permission from reference ${ }^{9}$.)

\subsection{Targeting mitochondria in cancer cells}

As mentioned above, mitochondria in cancer cells have become an attractive target with great interests. Therefore, it is quite necessary to develop mitochondrial targeting methodologies. Currently, mitochondrial targetingis achieved mainly based on different targeting molecules such as small peptides, proteins and chemical ligands. The development of mitochondrial targeting systems is to facilitate the delivery of small active molecules to mitochondria by crossing the double membranes (Figure 6) ${ }^{100}$.
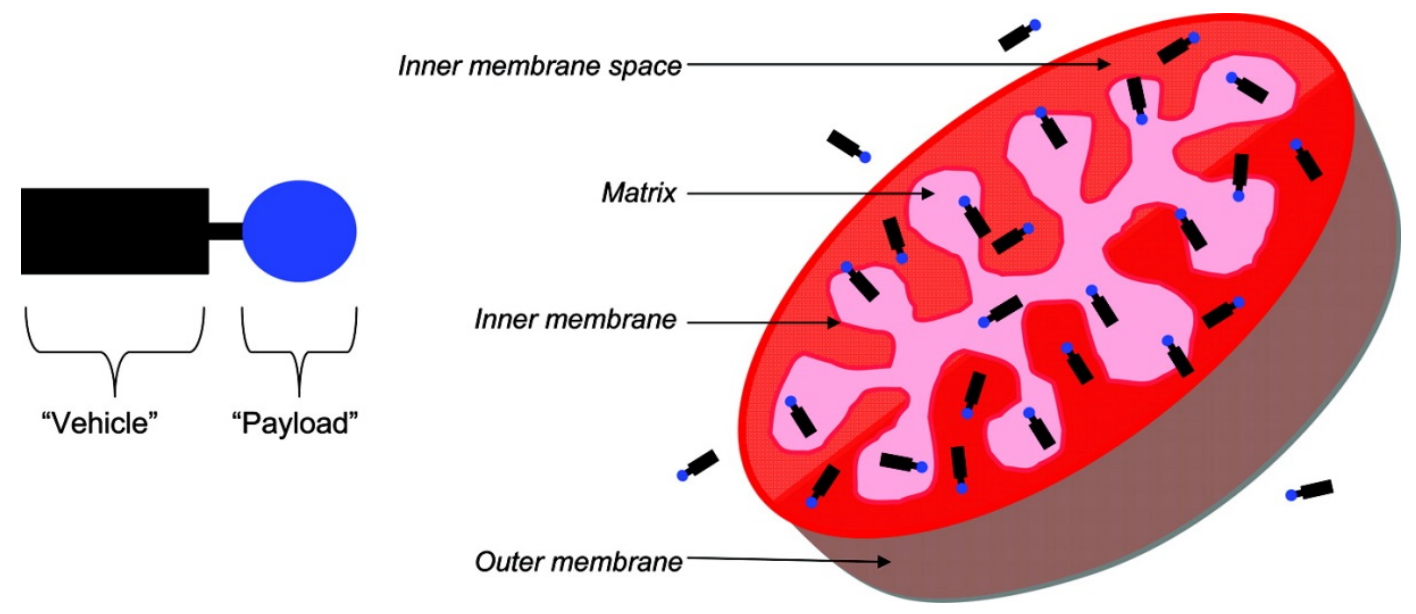

Figure 6. Concept of vehicle based mitochondrial targeted delivery system.(Reproduced with permission from reference ${ }^{100}$, Copyright 2008 American Chemical Society.)

\subsubsection{Mitochondrial targeting systems based on proteins}

Under normal cellular living conditions, the proliferation process of cell is achieved on the basis of variety of signal transductions and protein transportations. Generally, such cellular reactions are happening based on the interactions between different signal molecules and their receptors with specific chemical structures for recognition. As one of the most essential organelles, mitochondria based protein transportations are constantly occurring in order to supply materials to mitochondria from cytoplasm ${ }^{101,}{ }^{102}$. Therefore, there are number of 
polypeptides or proteins that are found to be able to target mitochondria and be recognized by mitochondria. Basically, proteins that are to be recognized and imported into mitochondria are often synthesized in the cytosol with specific amino acids sequence which can be recognized by mitochondria ${ }^{103}$. When such proteins interact with mitochondria, they are bound with translocases located at the outer and inner mitochondrial membrane, which can facilitate the transportation process of target proteins across the lipid bilayers.

In general, it is found that the target proteins to be imported and recognized by mitochondria have functional structure in $\mathrm{N}$ terminal or less frequently, $\mathrm{C}$ terminal for directing the transportation and recognition ${ }^{104}$. The $\mathrm{N}$ terminal or $\mathrm{C}$ terminal is generally consists of $20-30$ amino acids which can be cleaved by mitochondrial processing peptidase when being transported or in the mitochondrial matrix. In addition, when we compare the chemical structures of known $\mathrm{N}$ terminal or $\mathrm{C}$ terminal sequences, however, they do not share a common primary sequence but a common secondary structure including certain basic, hydrophobic as well as polar residues. Interestingly, some proteins such as cytochrome c and superoxide dismutase can be directly imported with minimal processing because they have sufficient structural elements necessary for recognition in their secondary structures ${ }^{105}$. Additionally, apart from the structural requirements necessary for protein recognition and transportation, another feature that is important for importing, is the localized positive charge from basic residues ${ }^{106}$. Since there is negative charge in the mitochondrial matrix, electrostatic force comes into play between mitochondria and target proteins. Consequently, the target proteins can be pulled across the mitochondrial inner membrane by the quite large membrane potential.

\subsubsection{Targeting mitochondria based on peptides and peptide mimics}

Up to date, a number of mitochondrial targeting peptides and peptide mimics have been 
developed and investigated for their targeting ability, including Szeto-Schiller (SS) peptides and XJB peptide mimics ${ }^{107}$.
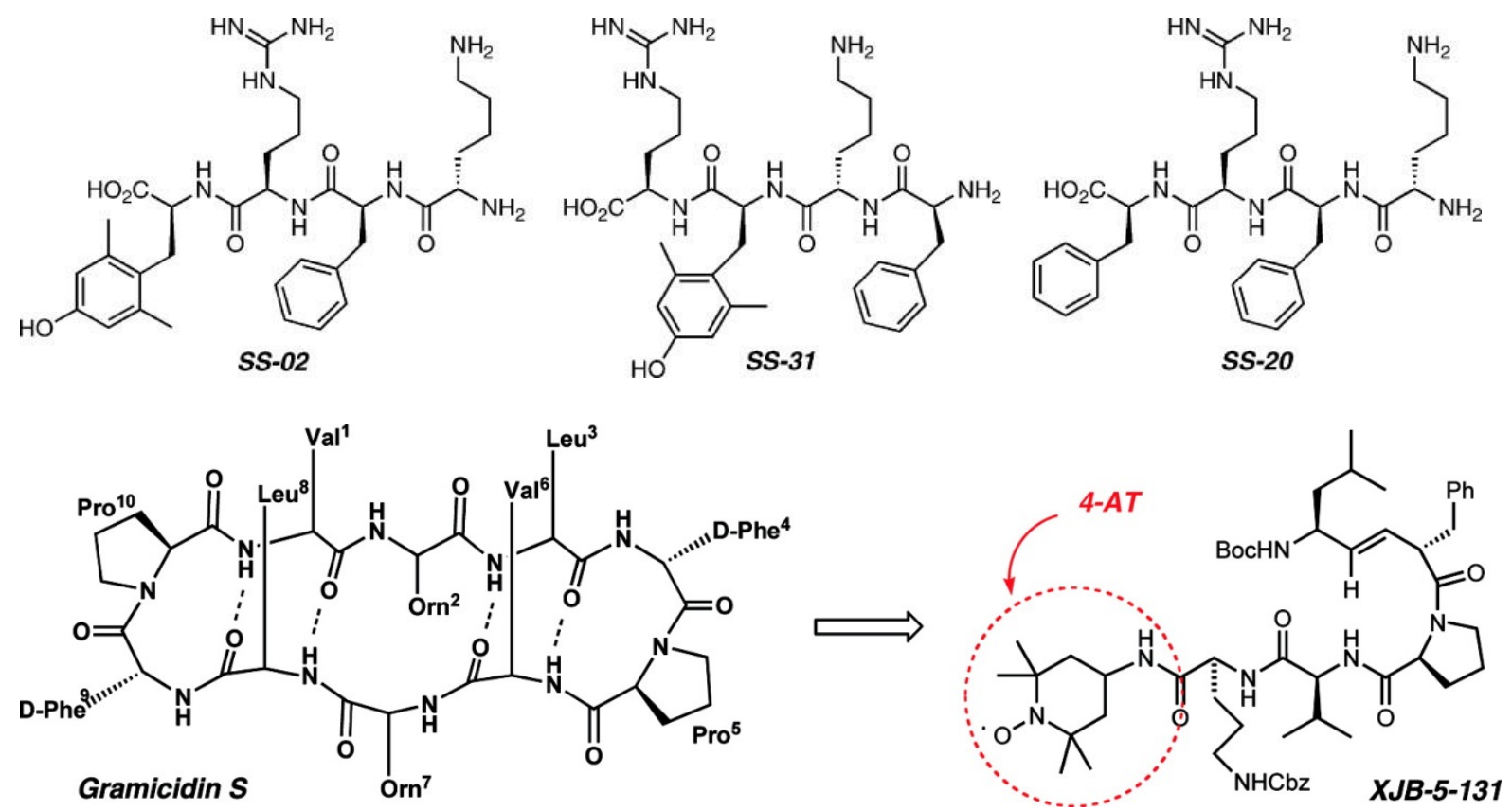

Figure 7.Chemical structures of different SS peptides (up) and XJB peptide (down).(Reproduced with permission from reference ${ }^{100}$, Copyright 2008 American Chemical Society.)

The SS tetra-peptides are a series of peptides that have the capability of mitochondrial targeting. In addition, SS peptides are also peptides with antioxidant property. They share a common structure of basic and alternating aromatic residues. Before discovery of their antioxidant and mitochondria targeting properties, SS peptides were extensively investigated for their selectivity and affinity to the $\mu$-opioid receptor ${ }^{108}$. Different structures of SS peptides are classified with different numbers. When considering the structure of SS-02 and SS-31, it is proposed that the di-methyltyrosine residues are critical for their antioxidant properties. In addition, SS-02 and SS-31 are found to effectively and equally scavenge $\mathrm{H}_{2} \mathrm{O}_{2}$ and inhibit linoleic acid oxidation in vitro. The net positive charge is caused by the basic residues, which facilitates the translocation of SS peptides across inner mitochondrial membrane. The dimethyltyrosinephenol moieties of SS-02 and SS-31 are thought to be responsible for reducing 
level of reactive oxygen species (ROS) ${ }^{109}$. Such function of di-methyltyrosinephenol structure is demonstrated by testing SS-20, where di-methyltyrosineis substituted with a phenylalanine residue. No ROS scavenging property was found in SS-20, indicating the important function of di-methyltyrosinephenol moiety in reducing ROS level. tertButylhydroperoxide (t-BHP) is an effective oxidant in introducing oxidative stress and apoptosis. t-BHP is membrane permeable and able to effectively trigger apoptosis by mitochondrial permeability transition (MPT), which dramatically increases the permeability of mitochondrial membrane to small molecules. Based on numbers of in vitro studies, peptides SS-02 and SS-31 have the ability to inhibit the triggering of apoptosis and reduce the permeability of mitochondrial membrane in t-BHP treated cells ${ }^{110}$. Additionally, it is reported that peptides SS-02 and SS-31 were able to significantly improve contractile force and prevent myocardial stunning in an ex vivo reperfusion study of guinea pig heart. It is thought that reperfusion injury can induce mitochondrial permeability transition and ROS overproduction. In contrast, it is found that SS-20 was unable to prevent myocardial stunning because of the lack of dimethyltyrosine moiety. Furthermore, peptide SS-02 was demonstrated to be effective in preventing myocardial stunning in rats, further proving that ROS level is important in myocardial stunning induced by reperfusion ${ }^{111}$.

The designing of XJB peptides and peptide mimics is based on the structure of membrane active gramicidin S (GS) antibiotics. The antioxidant properties of XJB peptides were thought to because of the attachment of the stable free radical, 4-amino-TEMPO (4-AT). Similarly, different structures of XJB peptides are classified with different numbers. It is documented that XJB-5-125 and XJB-7-75 showed effective inhibition of superoxide overproduction in mouse embryonic cells, whereas the control peptides with slightly altered structure, XJB-5-197 and XJB-5-194 showed no such protective effect. Furthermore, peptide XJB-5-131 was developed by replacing an amide bond with an alkene group, leading to the 
increase of biocompatibility. Moreover, more follow up studies were conducted in order to assess the capability of XJB-5-131 in prolonging the survival of rats ${ }^{112}$. It is found that XJB5-131 treated rats can survive dramatically longer after $55 \%$ loss of blood, demonstrating that the administration of a mitochondria targeting XJB-5-131peptide can have a significant physiological effect in animal model of critical illness ${ }^{113}$.

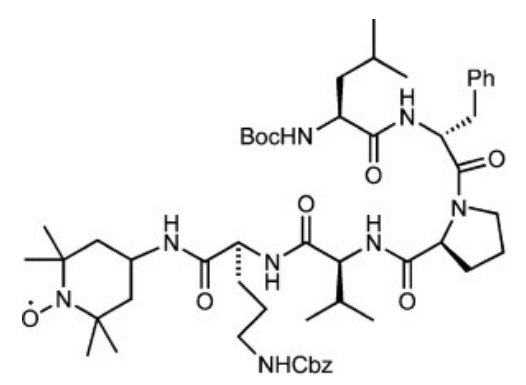

XJB-5-125<smiles>CC(C)CC(NC(=O)C(CCNC(=O)OCc1ccccc1)NC(=O)C(NC(=O)C1CCCN1C(=O)[C@@H](Cc1ccccc1)NC(=O)OCc1ccccc1)C(C)C)C(=O)NC1CC(C)(C)N(O)C(C)(C)C1</smiles>

XJB-7-75<smiles>CCCCNCC[C@H](NC(=O)C(NC(=O)[C@@H]1CCCN1C(=O)[C@H](Cc1ccccc1)NC(=O)[C@H](CC(C)C)NC(=O)c1ccccc1)C(C)C)C(=O)OC</smiles><smiles>COC(=O)C(CC(C)C)NC(=O)C(CCCNC(=O)OCc1ccccc1)NC(=O)C(NC(=O)C(C)C)C(C)C</smiles>

$X J B-2-300$<smiles>CON1C(C)(C)CC(NC(=O)C(CCNC(=O)OCc2ccccc2)NC(=O)C(NC(=O)C2CCCN2C(=O)[C@H](/C=C/C(CC(C)C)NC(=O)OCc2ccccc2)Cc2ccccc2)C(C)C)CC1(C)C</smiles>

XJB-5-131<smiles>CON1C(C)(C)CC(NC(=O)[C@H](/C=C/C(CC(C)C)NC(=O)OCc2ccccc2)Cc2ccccc2)CC1(C)C</smiles><smiles>CON1C(C)(C)CC(NC(=O)[C@H](CCNC(=O)OCc2ccccc2)NC(=O)C(NC(=O)C2CCCN2C(=O)[C@@H](C=C(C)C(CC(C)C)NC(=O)OCc2ccccc2)Cc2ccccc2)C(C)C)CC1(C)C</smiles>

XJB-5-197<smiles>COC(=O)[C@H](CCNC(=O)OCc1ccccc1)NC(=O)C(NC(=O)C1CCCN1C(=O)[C@H](/C=C/C(CC(C)C)NC(=O)OCc1ccccc1)Cc1ccccc1)C(C)C</smiles>

Figure 8.Various structures of other XJB peptides.(Reproduced with permission from reference ${ }^{100}$, Copyright 2008 American Chemical Society.)

\subsubsection{Targeting mitochondria based on non-peptide targeting species}

Mitochondria are uniquely structured with double membrane and more importantly and interestingly, the inner mitochondria membrane is constantly charged with around $-180 \mathrm{mV}$ across their lipid bilayer. The high negative membrane potential makes mitochondria very special and distinctive in any othercellular counterparts. Such unique property distinguishes 
mitochondria from other organelles and offers physical and chemical opportunities for mitochondrial targeting.

Based on mentioned special characteristic of mitochondria, lipophilic cations are selected to serve as selective mitochondrial targeting molecules ${ }^{87,96,114}$. Lipophilic cations can easily pass through lipid bilayers due to their dispersed charge over a large surface area and they can also accumulate in mitochondria matrix because of the driving force of the potential gradient and the electrostatic interaction ${ }^{115}$. Rhodamine 123 and the similar compounds containing cationic groups have the capability to cross the mitochondrial lipid membrane due to the electrostatic driving force, resulting in a 100-500 fold increase in accumulation according to Nernst equation ${ }^{116-119}$. Rhodamine 123 and similar compounds have been used as selective mitochondrial staining dye molecules as a result of their successful and reproducible selectivity towards mitochondria. Practical rhodamine-based mitochondrial staining assays have been developed and employed. In addition, Rhodamine 123 has also been used as chaperone to facilitate the transportation of tethered compounds into mitochondria ${ }^{120-124}$. By applying this method, the anticancer drug cisplatin was successfully imported into mitochondria.

Follow up the rhodamine 123 trials, the most beneficial discovery was the development of the utility of lipophilic triphenylphosphonium (TPP) salts, which are the majority of mitochondria targeting system based on non-peptide species. Two important features of TPP salts make them effective at targeting and delivering target cargo to mitochondria: TPP salts are able to freely cross phospholipid bilayers requiring no certain uptake mechanism and because of the large mitochondrial membrane potential, TPP salts can accumulate specifically and effectively within mitochondria ${ }^{125-127}$. To be specific, TPP salts molecules can easily cross through phospholipid bilayers, resulting from the fact that the activation energy requirement for TPP salts molecules when crossing lipid bilayers is much lower than other 
molecules. Here the activation energy is described as the energy required for transporting the cation from aqueous phase to the hydrophobic core of membrane, which is mainly consist of Born energy and the hydrophobic energy ${ }^{128-130}$. Generally, Born energy raises the activation energy whereas the hydrophobic energy lowers the activation energy. When the charge of the cation is determined, the Born energy is proposed to be inversely proportional to the radius of the cation. For the hydrophobic energy, it is the energy required for transporting an uncharged molecule with same size and hydrophobicity from aqueous phase into the core of lipid membrane. In general, the hydrophobic energy is proportional to the hydrophobic surface area of the cation. Therefore, with large hydrophobic radius, when crossing lipid bilayers, TPP cations have lower Born energy and larger hydrophobic energy, that is to say, TPP cations require lower activation energy when crossing lipid bilayers ${ }^{131}$.

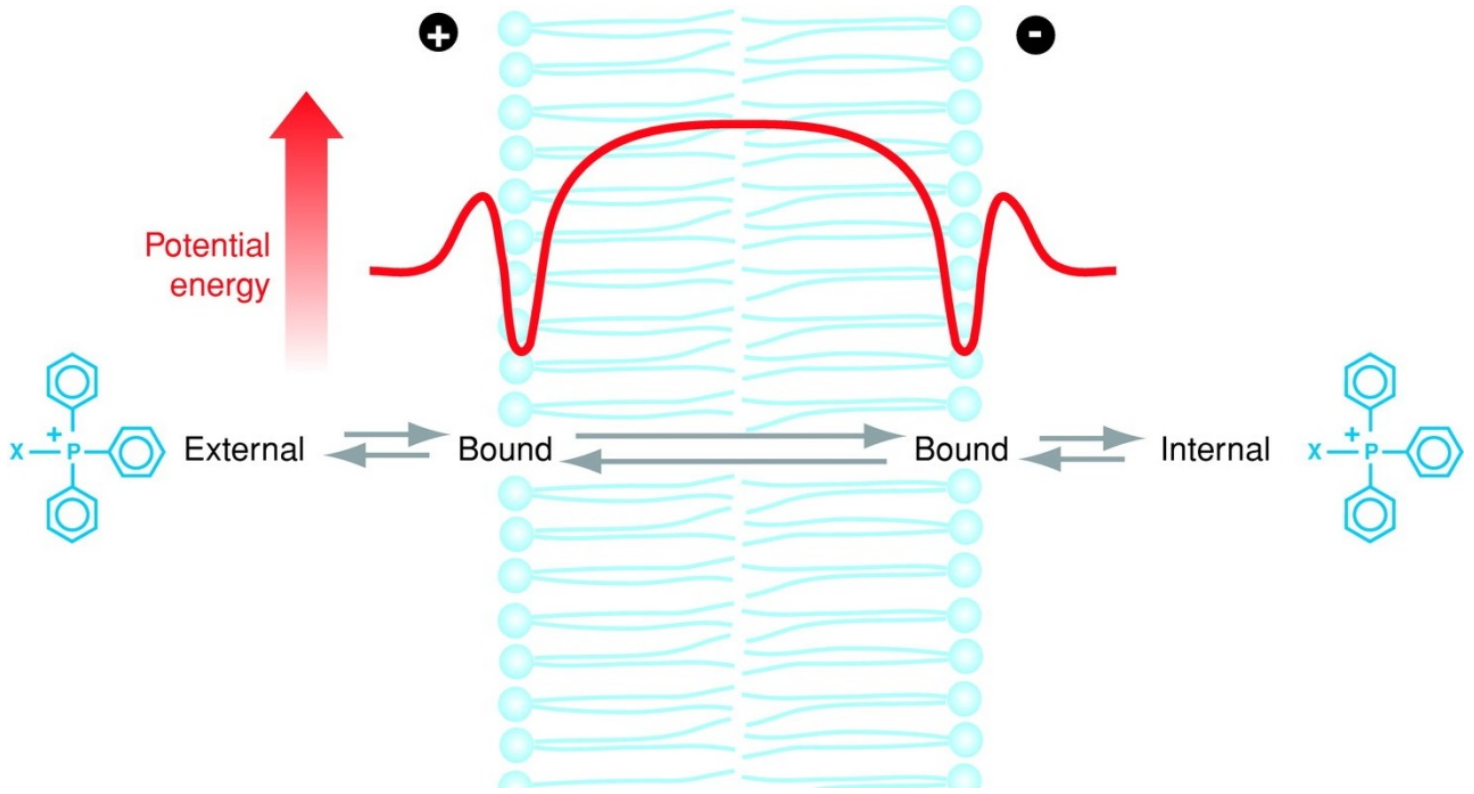

Figure 9. Schematic diagram of the uptake process of lipophilic cations through phospholipid bilayers.(Reproduced with permission from reference ${ }^{131}$, Copyright 2007 Annual Reviews.)

Beyond that, there are two energy potential wells close each membrane surface in terms of phospholipid bilayers (Figure 9). When TPP cations cross the phospholipid bilayers, they initially interact with the potential energy well on the outer surface of the membrane, then pass rapidly through the hydrophobic core to interact with the other potential energy well on 
the inner surface of the membrane, then further desorbing form the inner membrane. In addition to the factor of lowered activation energy requirement, another important factor that facilitates the targeting and importing of TPP cations to mitochondria is the force of electrostatic reaction (Figure 10). Typically, the mitochondria membrane potential in cells is $140-180 \mathrm{mV}$, resulting in the fact that cations in cytoplasm will accumulate specifically several hundred-fold within mitochondria ${ }^{132}$. In conclusion, the above mentioned two important features account for the effective targeting and delivering molecules into mitochondria.

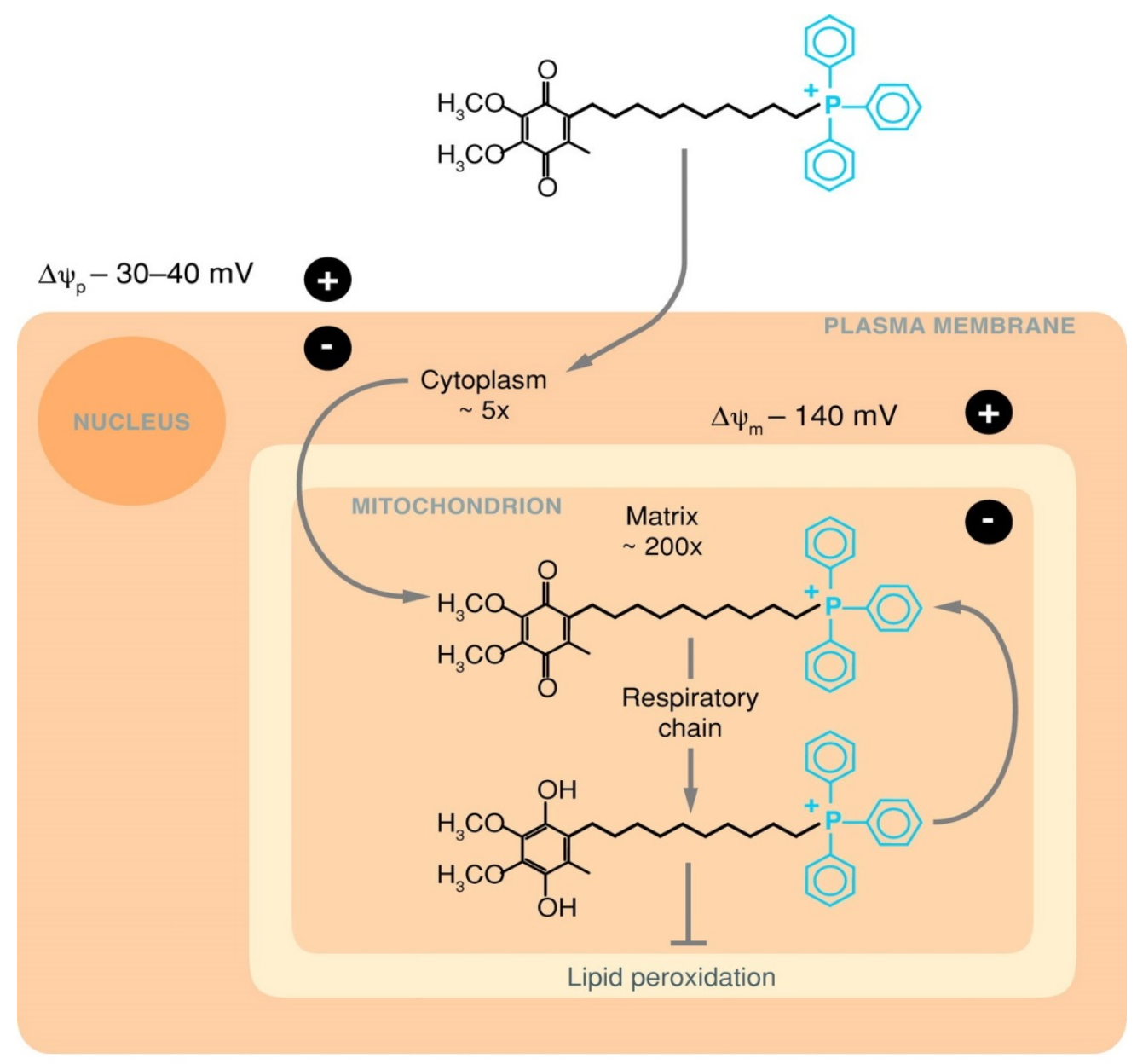

Figure 10. Diagram of the accumulation of TPP conjugated molecules (MitoQ ${ }_{10}$ ) in mitochondria driven by electrostatic interaction.(Reproduced with permission from reference ${ }^{131}$, Copyright 2007 Annual Reviews.)

It should be noted that apart from the effectiveness of TPP cations in targeting mitochondria, they are also commercially and cheaply available and can be easily chemically modified or 
conjugated with target cargo molecules or delivery systems. Therefore, in designing our mitochondria targeting system, we introduced the application of TPP cations with nanoparticle delivery system, the mesoporous silica nanoparticles (MSNPs).

\subsection{Mesoporous silica nanoparticles (MSNPs): development and application}

Highly ordered MSNPs have been discovered for more than 20 years by scientists at Mobil Corporation in 1992. Since then, MSNPs have been extensively investigated for different important applications ${ }^{133-136}$. Among these applications, designed MSNPs for effective delivery system is of the most significance because such function will be greatly achieved by the distinguished characteristics of MSNPs in terms of unique mesoporous structure, sufficient surface area for efficient loading amount of cargos and tunable pore size for incorporating cargos with various sizes (Figure 11) $)^{137}$. Furthermore, functionality on the surface of MSNPs can be easily conducted owing to the variety of possible chemical reactions. Thus, MSNPs can be the ideal delivery system for different $\operatorname{cargos}^{138-140}$.

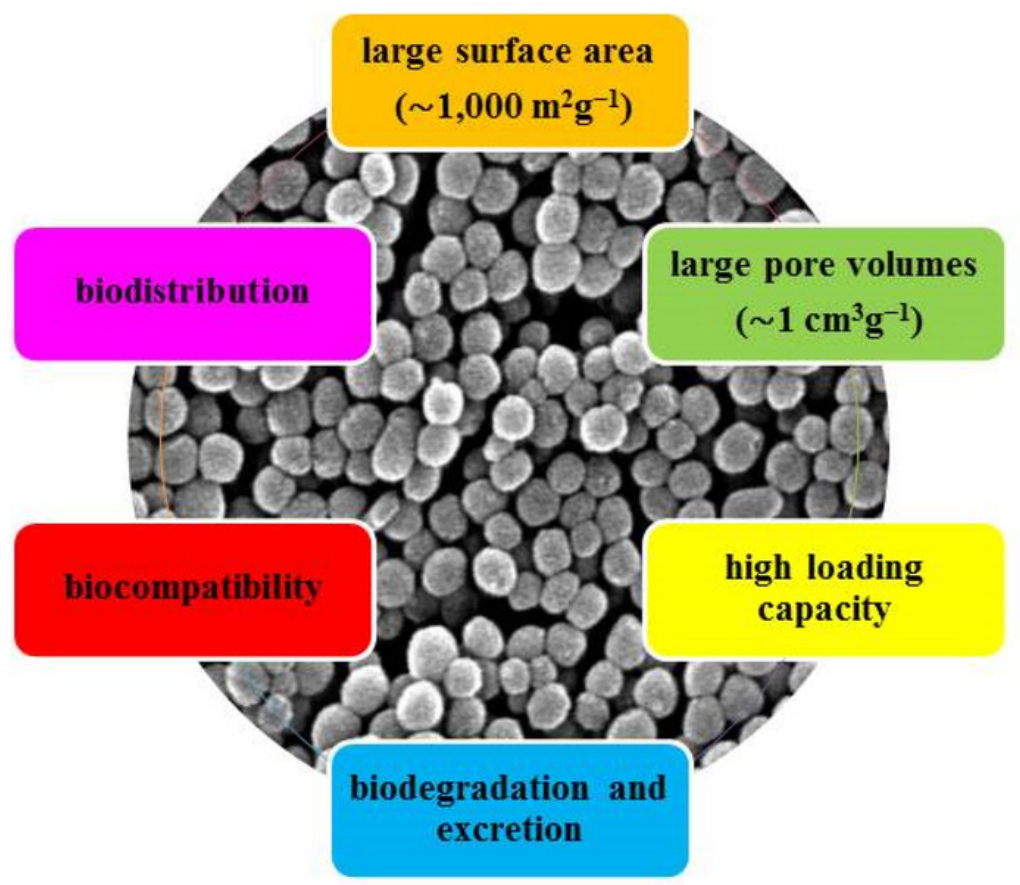

Figure 11.Attractive properties of MSNPs.(Reproduced with permission from reference ${ }^{137}$ ). 


\subsubsection{Synthesis and functionalization of MSNPs}

From the view of delivery application, the most commonly applied form of MSNPs is MCM-41 (Mobil Crystalline of Materials), which is typically consist of well-ordered cylindrical mesopores with hexagonal arrange. The general synthesis of MCM-41 includes the application of liquid crystal template, which commonly involves the use of cetyltrimethylammonium bromide $(\mathrm{CTAB})$. When treating $\mathrm{CTAB}$ molecules in aqueous solution, such amphiphilic surfactant molecule will self-assemble into spherical micelles and further into periodic liquid crystal meso-phases at higher concentrations ${ }^{141-144}$. When introducing the soluble silica precursors, the self-assembly of surfactant molecules will further form the hybrid nanocomposites. At the hydrophilic interfaces of micelles, silica precursor molecules will be concentrated and condensed through hydrogen bonding and electrostatic interactions to form the ordered silica mold of the periodic meso-phase. Finally, by subsequent removal of the surfactant template molecules by calcination or extraction methods, nanoparticles with mesoporous structure will be synthesized (Figure 12) ${ }^{145}$.

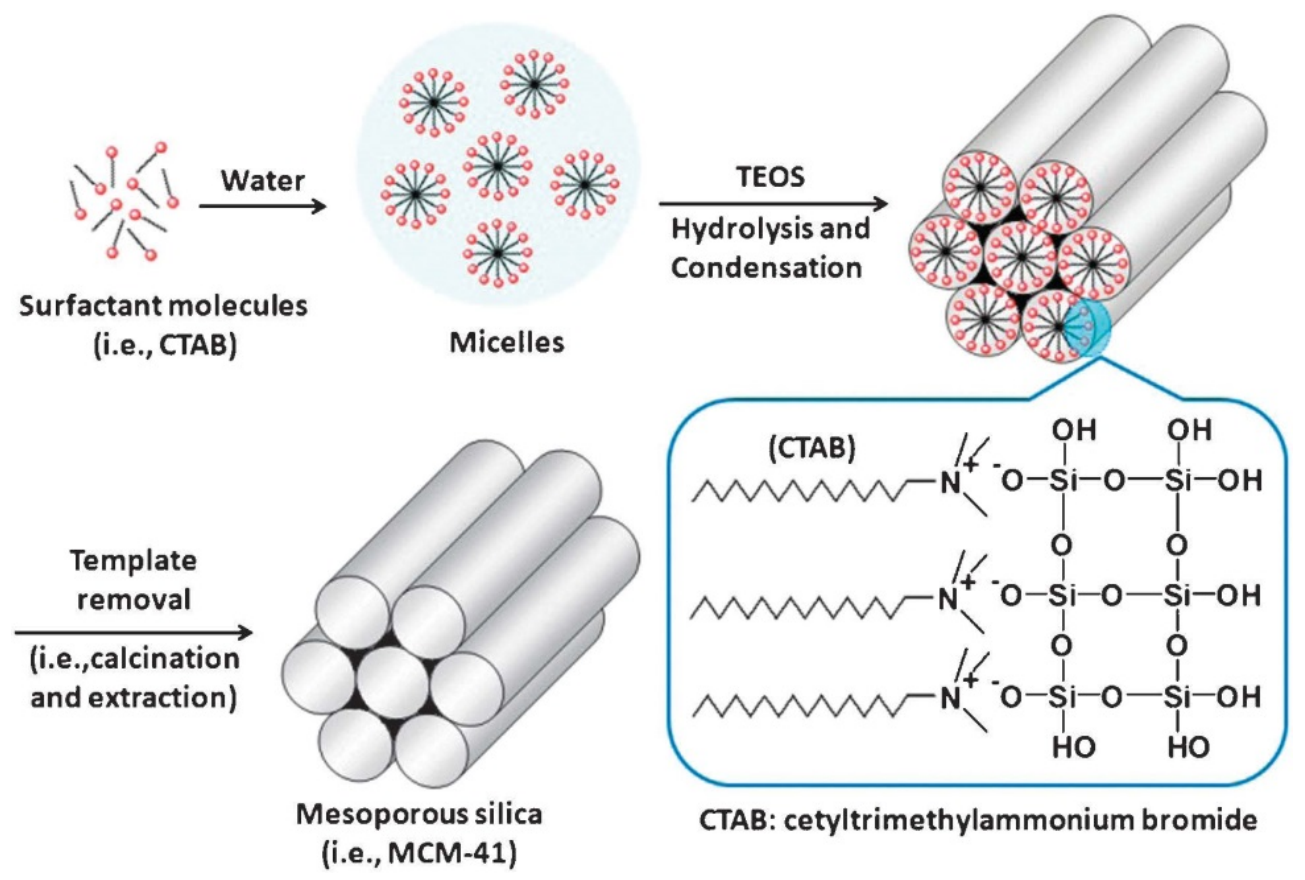

Figure 12.Schematic diagram of mechanism in fabrication of MCM-41 type of MSNPs directed by CTAB surfactant.(Reproduced with permission from reference ${ }^{145}$, Copyright 2012 


\section{Royal Society of Chemistry.)}

In order to achieve different functions of synthesized MSNPs, various surface modifications can be further conducted ${ }^{146},{ }^{147}$. Such surface modifications can be achieved by chemical interactions. The newly synthesized MSNPs can be functionalized by introducing different functional groups reacting with silanol groups present on the surface of MSNPs (Figure 13). It should be noticed that surface of MSNPs includes both the outer surface and the inner surface of MSNPs. Therefore, the modifications of MSNPs can be conducted on both outer and inner surface of MSNPs or only on the outer surface of MSNPs ${ }^{148}$. Because of the chemical accessibility of silanol groups, MSNPs can be easily functionalized. Generally, there are two major methods for MSNPs chemical modifications: co-condensation and postsynthetic grafting ${ }^{149-151}$. Co-condensation functionalization method is achieved by condensing hydrolyzed-alkoxysilanes together with organoalkoxy-silanes. Such co-condensing action results in the formation of MSNPs with direct functionalized on both inner and outer surface. The feature of amphiphilicity allows hydrolyzed organosilane to be a co-surfactant which is incorporated together with other surfactant such as CTAB to form the micelle structure. The mix-micelles are further condensed by silica, resulting in positioning the functional organic moieties directly on the walls of pores. Thus, we can see that co-condensation method of functionalization is a one-step functioning method. 


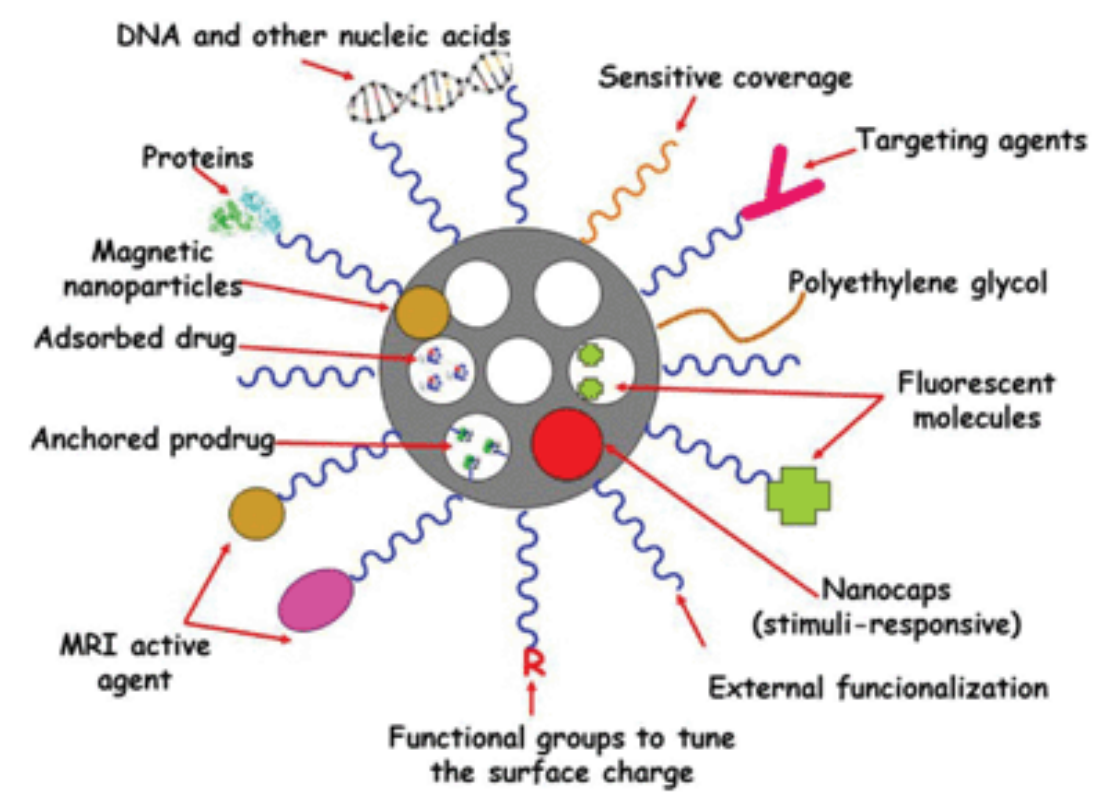

Figure 13.Illustration of multi-functionality on surface and cargo loading versatility of MSNPs. (Reproduced with permission from reference ${ }^{147}$, Copyright 2012 Royal Society of Chemistry.)

The second functionalization method is post-synthetic grafting, which means that the functionalization process is achieved after MSNPs synthesis ${ }^{152-154}$. This method just employs the chemical accessible silanol groups on both outer and inner surface of MSNPs. Nevertheless, it is found that the silanol groups located at the outer MSNPs surface and at the opening area of meso-pores are more chemically accessible than those located at deep inner surface of pores ${ }^{155-158}$. Therefore, it is shown that chemical functionalization of functional groups achieved by post-synthetic grafting method are mostly occurring on the outer surface and the opening areas of pores. Considering the characteristic synthetic method of MSNPs, however, post-synthetic grafting functionalization method has special advantage in selective functionalization. That is because the chemical grafting modification can be conducted prior to the removal of surfactant molecules from pores, resulting in the selective functionalization on the outer surface of MSNPs. Such selective surface modification may have great significance in that the interactions between MSNPs and environment can be achieved by modification without affecting the mesoporous structure of MSNPs. Furthermore, different other modifications of inner MSNPs surface can be conducted after surfactant removal to get 
functional groups different from those on outer MSNPs surface.

\subsubsection{Toxicity and bio-imaging application}

Owing to the characteristic properties of MSNPs including large surface area and volume, tunable particle and pore size and easy surface modification, they can be suitably applied in various biological applications. However, prior to talking about the biological applications, we need to discuss the critical issue of toxicity of MSNPs which is the pre-requisite for biological applications.

Considering the chemical structure of MSNPs, we need to think about the toxicity of silica and related molecules. Fortunately, the toxicity of crystalline and amorphous silicon dioxide has been fully studied many decades ago and in addition, the toxicity of silica nanoparticles has been extensively investigated recently as well ${ }^{159-163}$. It is found that silica nanoparticles may have notable toxicity towards cells, which is proposed to be caused by the high surface to volume ratio of silica nanoparticles that possibly leads to enhanced cellular membrane interactions as well as various pathways of toxicity ${ }^{164}$. In the case of MSNPs, however, the surface to volume ratio is much lower than that of silica nanoparticles. Therefore, the interactions between MSNPs and cellular membrane including hydrogen bonding and electrostatic interactions aresignificantly reduced, which means that the cellular toxicity of MSNPs is much lower than silica nanoparticles. Such conclusion can be supported by various recent investigations. For example, recent studies have observed that MSNPs exhibit much lower toxicity than corresponding silica nanoparticles without porous structure ${ }^{165}$. Such finding is presumably due to the decreased contacting area between MSNPs and cellular membrane. Also, it is reported that MSNPs could lead to reduced hemolysis of red blood cells.

In addition, it should be noted that the toxicity of MSNPs is highly cell specific, 
whichmeans that MSNPs is not always nontoxic to all cell lines. It is observed that RAW264.7 macro-phase cells were more sensitive to MSNPs with diameter of $115 \mathrm{~nm}$ than A549 cells, especially at high concentration of $250-500 \mu \mathrm{g} / \mathrm{mL}^{152}$. Furthermore, studies regarding application of MSNPs in vivohave also been conducted ${ }^{166}$. It is demonstrated that coating with polymers, proteins or lipid bilayers can efficiently reduce the toxicity of MSNPs in vivo. Importantly, it should be noticed that when discussing effective drug delivery, we need to think about the potential therapeutic concentration. For example, it is found that the LC50 and LC90 values of free doxorubicin (DOX) for Hep3B (hepatocellular carcinoma cell line) are $150 \mathrm{ng} / \mathrm{mL}$ and $500 \mathrm{ng} / \mathrm{mL}$, which can be delivered by MSNPs with concentration of $400 \mathrm{ng} / \mathrm{mL}$ and $1300 \mathrm{ng} / \mathrm{mL}$, respectively. Whereas when applying targeted MSNPs, the values above are $6 \mathrm{ng} / \mathrm{mL}$ and $20 \mathrm{ng} / \mathrm{mL}$ resulted from the effectiveness, stability and internalization efficiency of MSNPs ${ }^{167}$, 168. In conclusion, MSNPs show relatively much lower toxicity than solid silica nanoparticles and have acceptable toxicity both in vitro and in vivo.

After discussing the toxicity of MSNPs, we found that MSNPs have good biocompatibility, which makes them suitable in biological applications. So far, such applications mainly involve bio-imaging and cargo delivery. The bio-imaging application of MSNPs is achieved on the basis of good biocompatibility, which ensures that MSNPs can be effectively internalized by target cells and further fulfill their functions ${ }^{169-172}$. Typically, the purpose of bio-imaging mainly involves the visualization of location, size and status of target cells as well as interactions between different target cells. Although bio-imaging is not only for cell imaging, here we mainly discuss cell imaging with MSNPs. In most cases, bio-imaging using MSNPs is achieved by detecting fluorescent signal. Therefore, MSNPs need to be functionalized with fluorescent molecules. The most commonly applied fluorescent compounds include fluorescein iso-thiocyanate (FITC) and rhodamine B iso-thiocyanate 
(RITC). Such fluorescent compounds are often incorporated inside MSNPs to serve as labeling or staining compounds and further give fluorescent MSNPs. Some other dye molecules are utilized as well including near-IR dyes such as DyLight680 and AlexaFluor700. The above mentioned fluorescent molecules are fairly robust and can be easily incorporated inside MSNPs, leading to the formation of fluorescent MSNPs with capacity of generating high resolution and multichannel images. Additionally, the fluorescent MSNPs can also be used in obtaining quantitative data by using flow cytometry. Alternatively, another relatively new method for bio-imaging using MSNPs is to introduce magnetic nanoparticles such as magnetite $\mathrm{Fe}_{3} \mathrm{O}_{4}$, serving as the core of MSNPs. Such design enables MSNPs for $\mathrm{T}_{2}$-weighted MRI imaging. In addition, by incorporating chelated gadolinium compound, MSNPs can also be used in $\mathrm{T}_{1}$-weighted MRI imaging ${ }^{173,174}$.

\subsubsection{Delivery application}

Apart from applying MSNPs in bio-imaging, another important biological application of MSNPs is delivering target cargo molecules. Several characteristic properties of MSNPs make them quite suitable for delivery application, including large surface area and volume, tunable particle and pore size and easy surface functionalization. In general, MSNPs have quite large surface area of 700-1500 $\mathrm{m}^{2} / \mathrm{g}$ and the meso-pores are tunable in diameter ranging from 2 to $30 \mathrm{~nm}$. Surface modifications with different chemical ligands are of great importance and necessity in developing MSNPs to be effective delivery platform ${ }^{136,175-}$ ${ }^{179}$.The surface modificationsof MSNPsare mainly aiming at enhancing the specific cellular internalization while limiting the extent of non-specific binding, resulting in the specific targeting of MSNPs to intended regions. Plus, targeting property of MSNPs plays an important role in enhancing overall biocompatibility. Up to date, the developed specific targeting of MSNPs involves passive targeting as well as active targeting. Basically, the 
passive targeting of MSNPs is achieved on the basis of enhanced permeability and retention (EPR) effect (Figure 14), leading to the direct accumulation of nano-carriers at intended tumor site ${ }^{180}$.
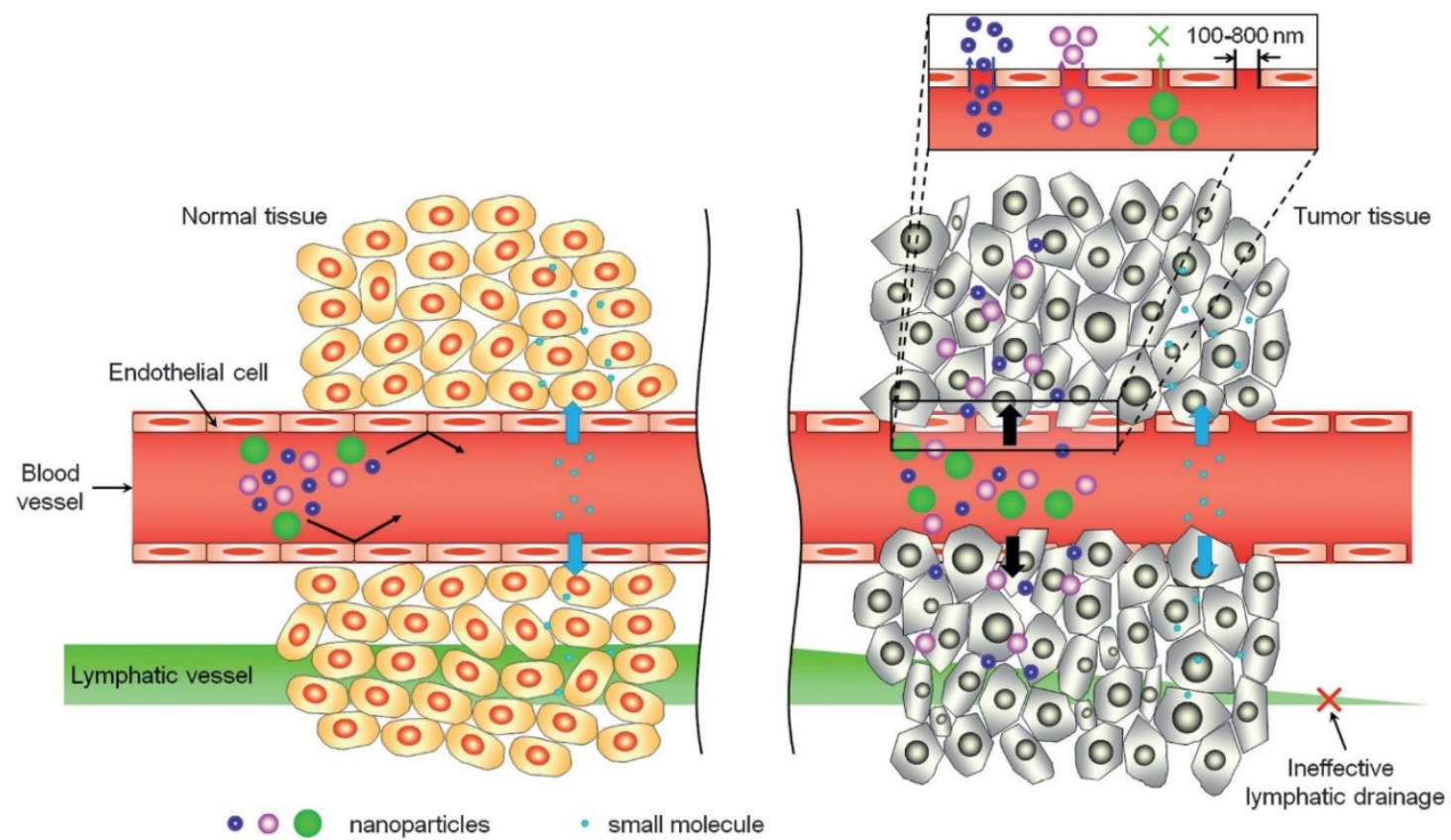

Figure 14.Schematic illustration of EPR effect, which results in the passive accumulation of therapeutic agents in tumor tissue rather than normal tissue.(Reproduced with permission from reference ${ }^{179}$, copyright 2015 Nature Publishing Group.)

In addition, passive targeting of MSNPs can be further enhanced by biocompatible polymer coating. For example, it is demonstrated that the cellular internalization of MSNPs can be significantly increased by coating MSNPs with cationic polymers such as poly-ethylenimine $(\mathrm{PEI})^{181}$. Also, through polyethyleneglycol (PEG) and PEI copolymer coating, MSNPs can exhibit enhanced EPR effect on a xenograft model. The active targeting of MSNPs involves the strategy of employing selective targeting ligands that specifically interact with certain receptor molecules on cell surface with interest to promote the binding and internalization of MSNPs. Such strategy requires that certain receptors need to be highly over-expressed by cancerous cells comparing with normal cells. According to this strategy, numerous bioactive molecules are employed owing to their corresponding receptors being overexpressed in many types of cancerous cells, including RGD peptide, folate acid and transferrin ${ }^{182-187}$. Generally, 
the achievement of high biding affinity of MSNPs and target cells is based on the high concentration of targeting ligands conjugated on the surface of MSNPs, which can lead to more sufficient of cargo delivery through receptor mediated internalization pathways. High surface-conjugated ligands on the surface, however, may promote interactions between MSNPs and endothelial and other noncancerous cells, which may result in the increase of immunogenicity of MSNPs. Regarding this issue, researchers have found that solutions can be obtained by constructing MSNPs with lipid bilayer coating ${ }^{147,165}$.

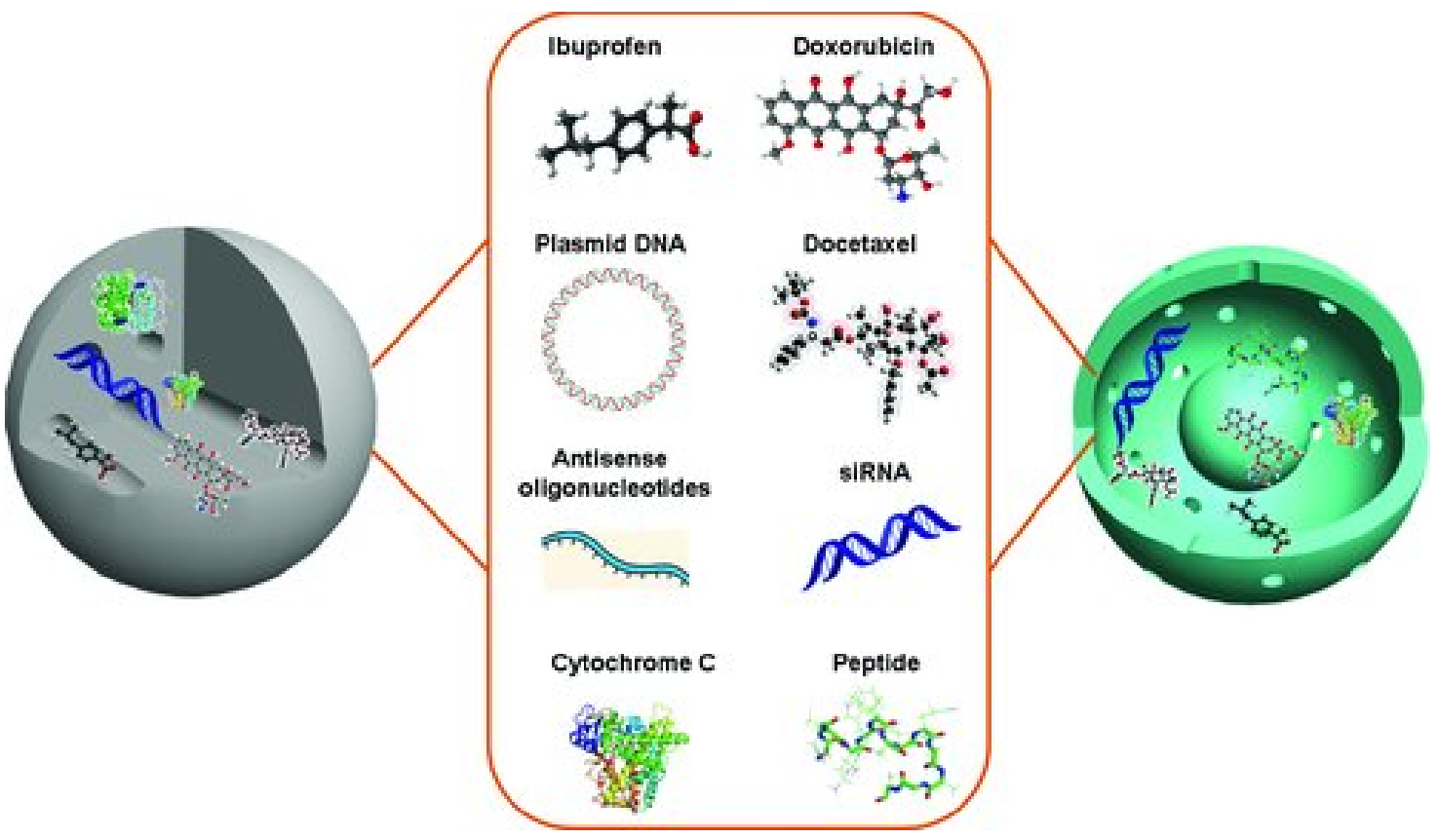

Figure 15.MSNPs as effective drug delivery system for various cargos including therapeutic genes (siRNA and plasmid DNA), pharmaceutical drugs (Ibuprofen, Doxorubicin and Docetaxel) and therapeutic peptides and proteins such as cytochrome c.(Reproduced with permission from reference ${ }^{142}$, Copyright 2012 WILEY-VCH Verlag GmbH \& Co. KGaA, Weinheim).

After the discussion of designing MSNPs with targeting property, we need to further talk about another important aspect: cargo molecules loading and delivery. Due to the sufficient surface area and volume and tunable pore size, MSNPs can be suitably employed in incorporating and loading different target cargo molecules (Figure 15) ${ }^{142}$. Studies in early stage of using MSNPs in drug delivery focused on molecules with poor water solubility, including ibuprofenandaspirin in particular. Later, more and more studies have been focusing 
on delivering hydrophobic molecules such as camptothecin, to cancerous cells using MSNPs to perform cancer treatment. It is quite advantageous for MSNPs to perform delivery of hydrophobic molecules in that hydrophobic compounds can be loaded into the pores of MSNPs from non-aqueous solutions and retained in aqueous environments. After endocytosis process, the loaded hydrophobic molecules can be further released in the complex cellular micro-environment. In addition, further surface modifications may be needed for MSNPs when incorporating hydrophilic molecules. For example, it is observed that after introducing negatively charged groups onto the surface of MSNPs, loading of one of hydrophilic drug molecules, DOX, can be enhanced.

\subsubsection{Stimuli-responsive control release}

For effective nano-carriers, more importantly, the loaded cargo molecules must be retained within nano-carriers and released when reaching the target site, the so-called controlled release. In the case of MSNPs, such requirement can be achieved by the robust absorption of cargo molecules or sealing the pores after cargo incorporating. One method that is employed in pore sealing includes the introduction of capping compounds such as quantum dots. With such capping compounds, loaded cargo molecules can be sealed by blocking the entrance of pores. As for the controlled release of cargo molecules, a number of stimuli responsive strategies have been developed (Figure 16). Those strategies mainly involve the consideration of the environmental changes after loaded MSNPs being delivered to target site ${ }^{137}$.

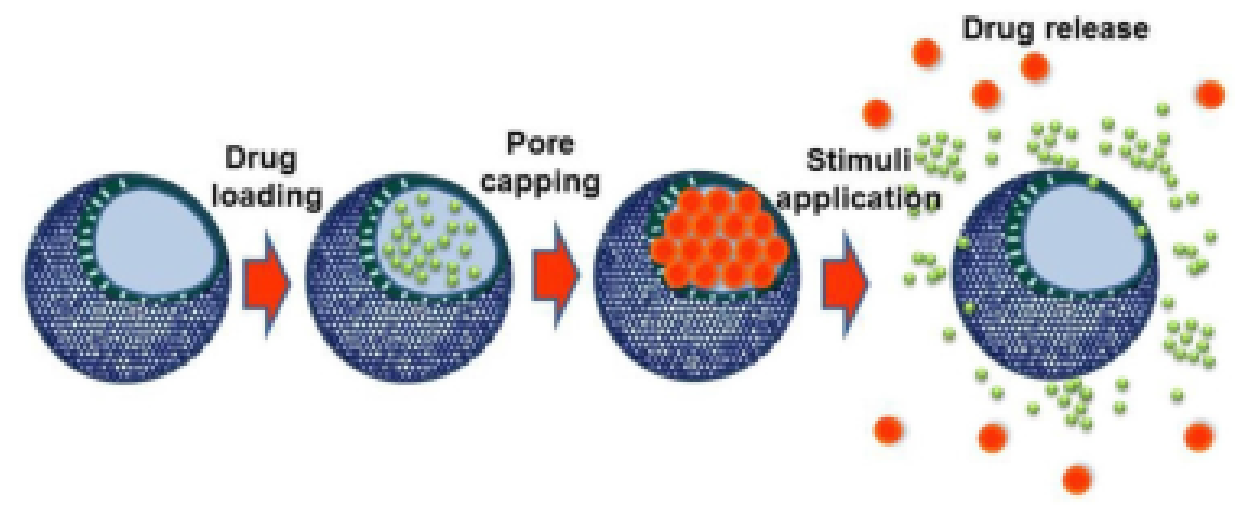


Figure 16. Schematic diagram of control release and stimuli responsive properties of MSNPs. (Reproduced with permission from reference ${ }^{137}$ ).

One common example is the $\mathrm{pH}$ responsive MSNPs ${ }^{188-190}$. Those MSNPs are designed by conjugating $\mathrm{pH}$ sensitive chemical groups onto the surface. When the environmental $\mathrm{pH}$ value changes, such surface-conjugated groups can be cleaved, leading to the release of cargo molecules from pores. Another external stimuli that can be applied in control release from MSNPs is reduction level. For example, it has been developed that MSNPs surface functionalization with chemically cleavable disulfide linkage can achieve the controlled release since that such disulfide linkage can be easily cleaved by the increased reduction level within the cancerous microenvironment. A controlled release model based on MSNPs has been reported that MSNPs were loaded with vancomyocin and adenosine 5-triphosphate(ATP) and the pores were further capped by $\mathrm{CdS}$ nanoparticles with $2.0 \mathrm{~nm}$ diameter as chemically removable capping compounds ${ }^{191},{ }^{192}$.After that, disulfide amine functional groups were further chemically functionalized on the surface of MSNPs as responsive linkage which can be reduced by various reducing agents such as mercaptoethanol (ME) and dithiothreitol (DTT). And the cleavable ability of disulfide linkage conjugated on the surface by reducing agents was further demonstrated (Figure 17) ${ }^{193}$. Such system design based on reduction responsive linkage is come up with the fact that the reduction level within microenvironment in cancerous cells is higher than that in normal cells. 


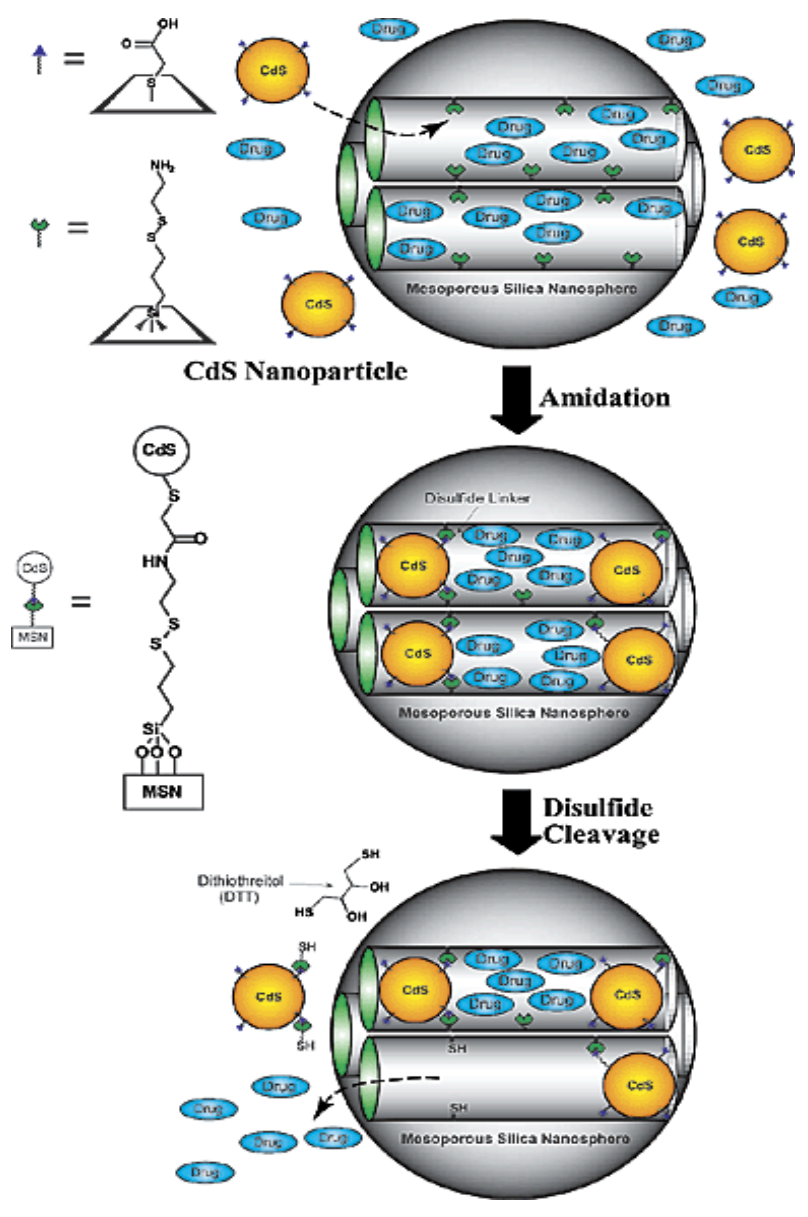

Figure 17. Illustration of CdS capped MSNPs in controlled drug delivery.(Reproduced with permission from reference ${ }^{193}$, Copyright 2003 American Chemical Society).

Besides the application of CdS nanoparticles as pore capping compound, other nanoparticles like super-paramagnetic iron oxide $\left(\mathrm{Fe}_{3} \mathrm{O}_{4}\right)$ can be utilized as well to achieve the avoidance of premature release of cargo molecules from MSNPs until reaching the intended target site. In addition to the utility of inorganic nanoparticles as capping compounds, it has been demonstrated that large organic molecules can be applied as modification of surface of MSNPs as well. This design is also aimed at achieving control release of cargo molecules. It has been reported that MSNPs were designed with plasmid DNA loading and capped with second generationpolyamidoamine (G2-PAMAM) dendrimer. The loaded plasmid DNA is responsible for coding green fluorescent protein (GFP) which can be expressed in cellular microenvironment. Additionally, MSNPs can prevent loaded plasmid DNA from digestion by enzyme. By detecting the expression of GFP which can be easily visualized by fluorescence 
microscopy, the successful delivery of plasmid DNA by MSNPs and control release of plasmid DNA can be further confirmed. It is also reported that pores on MSNPs can be capped with gold nanoparticles and such capped MSNPs are able to further deliver chemical molecules to plant cells ${ }^{92}$.

Apart from above mentioned chemical stimuli, another MSNPs-based system with electrochemical redox-activation property was also developed. The investigators designed a gate opening system based on a supra-molecular nano-valve conjugated to the opening area of meso-pores on the surface of MSNPs, which can be chemically turned on by redox reaction, leading to the opening of pores. MSNPs were loaded with fluorescent molecules and such molecules can be released when pores are opened by stimulation of external reducing agents. Following this research, investigators also developed an updated nano-valve that can be reversibly operated. Based on that, the pores on the surface of MSNPs can be turned on and off by redox chemistry. The reversibly designed nano-valve used a large molecule to cap the free end of the tether, therefore maintaining the interaction between cyclophane and the tether. The pores can be uncapped by the movement of cyclophane, resulting in the release of guest molecules. Through reduction reactions, the cyclophane can return to the opening area of pores and further recap the pores ${ }^{194}$. 


\subsection{Mitochondrial targeting agents in cancer therapy}

\begin{tabular}{|c|c|c|}
\hline Class & Compound & Target or mode of action \\
\hline \multirow{6}{*}{$\begin{array}{l}\text { Modulators of the } \\
\text { BCL-2 protein family }\end{array}$} & A-385358 & $B C L-X_{L}$ \\
\hline & ABT-263, ABT-737 & $B C L-2, B C L-X_{L}, B C L-W$ \\
\hline & AT-101 & BCL-2, BCL-X, BCL-W, MCL1 \\
\hline & GX15-070 (obatoclax) & BCL-2, BCL-X, BCL-W, MCL1 \\
\hline & HA14-1 & $\mathrm{BCL}-2$ \\
\hline & Oblimersen & $\mathrm{BCL}-2 \mathrm{mRNA}$ antisense \\
\hline \multirow[t]{9}{*}{ Metabolic inhibitors } & 2-Deoxy-D-glucose & HK \\
\hline & 3-Bromopyruvate & HK2-VDAC interaction \\
\hline & Dichloroacetate & PDK inhibitor \\
\hline & HK2 peptide & HK2-VDAC interaction \\
\hline & LDH-A shRNA & LDH-A \\
\hline & Methyl jasmonate & HK2-VDAC interaction \\
\hline & Orlistat & Fatty acid synthase \\
\hline & SB-204990 & ATP citrate lyase \\
\hline & Soraphen A & Acetyl-CoA carboxylase inhibitor \\
\hline \multirow{5}{*}{$\begin{array}{l}\text { VDAC-targeting } \\
\text { and/or ANT-targeting } \\
\text { agents }\end{array}$} & Arsenite trioxide & ANT ligand, ROS production \\
\hline & Clodronate & ANT inhibitor \\
\hline & GSAO & ANT cross linker \\
\hline & Lonidamine & ANT ligand \\
\hline & PK11195 & PBR ligand \\
\hline \multirow[t]{10}{*}{ ROS regulators } & 2-Methoxyestradiol & SOD inhibition \\
\hline & ATN-224 & SOD inhibition \\
\hline & $\beta$-lapachone & ROS production \\
\hline & Buthionine sulphoximine & GSH synthesis inhibitor \\
\hline & Imexon & GSH depletion \\
\hline & Mangafodipir & SOD mimic \\
\hline & Menadione & ROS production \\
\hline & Motexafin gadolinium & ROS production \\
\hline & PEITCs & GSH depletion, GPX inhibition \\
\hline & STA-4783 & ROS production \\
\hline \multirow[t]{3}{*}{ Retinoids } & All-trans-retinoic acid & ANT ligand \\
\hline & CD437 & Permeability transition pore complex \\
\hline & ST1926 & Perturbation of $\mathrm{Ca}^{2+}$ homeostasis \\
\hline \multirow[t]{3}{*}{ HSP90 inhibitors } & Gamitrinibs & Mitochondrial HSP90 ATPase inhibitors \\
\hline & PU24FCI, PU-H58, PU-H71 & HSP90 inhibitors \\
\hline & Shepherdin & Inhibitor of the HSP90-survivin interaction \\
\hline \multirow{5}{*}{$\begin{array}{l}\text { Natural compounds } \\
\text { and derivatives }\end{array}$} & $\alpha$-tocopheryl succinate & Ubiquinone-binding sites in respiratory complex II \\
\hline & Betulinic acid & Permeability transition pore complex \\
\hline & DMAPT & ROS production \\
\hline & Parthenolide & ROS production \\
\hline & Resveratrol & $\mathrm{F}_{1}$-ATPase \\
\hline
\end{tabular}

Table 1.Summary of active mitochondrial targeted compounds.(Reproduced with permission from reference ${ }^{195}$, Copyright 2010 Rights Managed by Nature Publishing Group).

Based on the premises that mitochondria are promising target sites in cancer treatment discussed above, pharmacological agents that can target mitochondria emerge as an attractive strategy in cancer therapy by inducing cancer-associated mitochondrial dysfunctions and activating the programmed cell death process. Some active compounds with different acting strategies are summarized (Table 1) ${ }^{195}$. 


\subsubsection{Agents that target mitochondrial permeability transition (MPT)}

Under normal physiological conditions, mitochondria exhibit negative charge due to the trans-membrane potential. The structure that is responsible for the exchange of small molecules between mitochondrial matrix and cytosol is called permeability transition pore complex (PTPC) which is a highly dynamic complex across the outer and inner mitochondrial membrane. In pro-apoptosis process, the structure of PTPC would be changed and the entry of small molecules into mitochondrial matrix is deregulated in response to preapoptotic signals including ROS generation and calcium ions over production, leading to the formation of MPT which is indicated by long time opening of PTPC and the increase of inner mitochondrial membrane permeability. Following that, MPT would result in the immediate losing of mitochondrial membrane potential. Eventually, MPT leads to MOMP formation and further apoptosis. Therefore, it would be a way for apoptosis activation and cancer therapy by inducing MPT and MOMP formation and several compounds have been found to exhibit such functions. 4-(N-(S-glutathionylacetyl)amino) phenylarsenoxide (GSAO), a compound that is found to interact with ANT structure part of PTPC, leading to the inhibition of ATP and ADP transportation, overproduction of ROS and further apoptosis ${ }^{196}$. Such GSAO molecules are shown to preferentially target proliferating cells like cancer cells because of their high level of mitochondrial calcium ions and elevated rate of respiration rate, making GSAO an ideal agent. Another compound called clodronate, one of bisphosphonates, is known to be able to act as competitive inhibitor of ANT, resulting in the decrease of oxygen consumption, losing of mitochondrial membrane potential and apoptosis. By adding of oral clodronate to patients with breast cancer, the survival rate of primary breast cancer patients increased $^{197}$. 


\subsubsection{Agents that induce ROS overproduction}

Intracellular overproduction of ROS is one of the main causes of intracellular oxidative stress, aging and apoptosis. In general, ROS are reactive chemical species containing oxygen including superoxide, hydroxyl radical and singlet oxygen. ROS are the natural byproducts of normal metabolism of oxygen and the intracellular oxidative level is balanced between ROS and anti-oxidant level. However, under environmental stress such as UV and heat exposure, ROS level can be dramatically increased, leading to cell damage.

$\beta$-lapachone (ARQ 501) is another compound that could reduce ROS production ${ }^{198}$. Clinical investigation of $\beta$-lapachoneis being currently conducted in combination with other anticancer compounds in patients with pancreatic cancer. In addition, buthioninesulphoximine is a compound that is able to indirectly accumulate ROS by inhibiting the production of reduced glutathione (GSH) which is the major intracellular anti-oxidative molecule. Buthioninesulphoximine has been investigated in Phase I clinical trial with patients with breast and lung cancer. Compound mangafodipir is a mimic of superoxide dismutase (SOD) with activities of glutathione reductase and catalase. Leukaemiais known as one of the most deadly diseases in human beings. By affecting the intracellular oxidative stress, there are several biological active compounds that may be suitable for the treatment of leukaemia. Compound 2-methoxyoestra diol is one of oestrogen derivatives, which is known for its killing effectiveness and selectivity over human leukaemia cells by induction of intracellular superoxide and SODinhibition ${ }^{199}$. Another active therapeutic compound called arsenic trioxide is known for its effectiveness in the treatment of acute promyelocyticleukaemia as well as many other solid tumors by inhibiting the activity of thioredoxin reductase and further elevating the intracellular oxidative stress ${ }^{200}$. 


\subsubsection{Agents that target mitochondrial metabolism}

Under normal physiological conditions, metabolism in normal cells mostly depends on the supply of oxygen and ATP production by oxidative phosphorylation. While in cancer cells, metabolism and energy supply mostly rely on the high rate of glycolysis process despite high level of oxygen. In addition, the electron flow in respiration chain in cancer cells is lower than that in their normal cells. Accordingly, agents that can reverse the hyper-glycolytic condition in cancer cells and induce apoptosis are promising in the development of selective anti-cancer therapies. 3-Bromopyruvate (3-BP) is a compound that is known for its pronounced anti-cancer efficiency in pancreatic and hepatic cancers in vivo. 3-BP is the inhibitor of hexokinase (HK), which is a protein interacting with PTPC, thereby preventing mitochondrial membrane permeabilization. It is found that $\mathrm{HK}$ is usually over-expressed in cancer cells and the binding between HK and PTPC is stronger in cancer cells than in normal cells. Thus, disrupting the interaction between HK and PTPC at outer mitochondrial membrane is the strategy that has been demonstrated to selectively kill cancer cells. By inhibiting the binding between HK and PTPC, 3-BP shows promising anti-cancer effect ${ }^{201}$.

Another compound called dichloroacetate, is known to be able to reverse the high rate of glycolysis in cancer cell to glucose oxidation, further leading to cell death. Additionally, as an inhibitor of mitochondrial pyruvate dehydrogenase kinase (PDK), dichloroacetatehas also been shown to reduce the high mitochondrial membrane potential, resulting in the overproduction of ROS. Such effect, however, may not happen in normal cells. Lactate dehydrogenase A (LDHA) is an enzyme that catalyzes the inter-conversion of NADH and $\mathrm{NAD}^{+}$as well as pyruvate and L-lactate. It is found that knockdown of LDHA by biological method like short hairpin RNAs would lead to decreased mitochondrial membrane potential, increased rate of mitochondrial respiration and further apoptosis ${ }^{202}$. 


\subsubsection{Other agents that target mitochondria in cancer cells}

Heat-shock proteins (HSPs) are proteins that are produced by cells in response to environmental stresses such as UV, temperature and wound healing. HSPs are evolutionarily conserved and have important functions for example stabilizing new proteins and refolding proteins that are damaged in stressful conditions. One of HSPs, HSP90, interestingly, is contained in mitochondrial in cancer cells while not in their normal counterparts ${ }^{203}$. It has been found that HSP90 can interact with PTPC and control the formation of MPT as well as mitochondrial signaling by protein folding mechanism. Therefore, HSP90 inhibitors can be exploited to affect signaling network in mitochondria. Shepherdin is among one of such HSP90 inhibitors. In addition, Gamitrinibs, described as GA mitochondrial matrix inhibitors, have been discovered presenting mitochondrial toxic, leading to cancer cell death and tumor suppression while not in normal cells ${ }^{204}$.

In addition, some naturally discovered compounds are also found to present mitochondria targeting property as well as mitochondrial toxicity. Betulinic acid is natural product found in various plant species, which can trigger apoptosis process selectively in cancer cells. When betulinic acid is directly introduced to isolated mitochondria out of cellular microenvironment, direct triggering of MOMP along with release of cytochrome $\mathrm{c}$ and mitochondrial membrane potential dissipation were observed ${ }^{205}$. It is also suggested that the effective anti-cancer effect of betulinic acid is achieved by targeting MPT, which is thought to be initiated by the overproduction of ROS. What's more, betulinic acid molecules are capable of modulating the expression level of BCL-2 family proteins ${ }^{206}$. They can up-regulate the BAX and other pro-apoptotic members. More importantly, it is also observed that in some cell types, the expression level of myeloid leukemia cell differentiation protein (MCL1) is closely related with betulinic acid. However, in carcinoma cells and squamous cells, no changes in MCL1 expression level were observed when cells were treated with betulinic acid. 
Additionally, apoptosis triggered by betulinic acid is not associated with the level of p53 protein.

Other kind of naturally discovered compounds are vitamin E analogues. Within vitamin E analogues, $\alpha$-tocopheryl succinate ( $\alpha$-TOS) is thought to be the most effective compound in selective triggering apoptosis in tumor cells. As a derivative of $\alpha$-tocopheryl $(\alpha-\mathrm{TOH}), \alpha$-TOS molecules are commonly achieved by substituting the hydroxyl group at position $\mathrm{C}_{6}$ by succinic acid group. Through genetic, biochemical and molecular modelling studies, the mechanism of anti-cancer effect of $\alpha$-TOS is the interaction between $\alpha$-TOS molecules and distal and proximal ubiquinone-binding sites in respiratory complex II, leading to the translocation of ubiquinone from complex II, followed by the combination of molecular oxygen and electrons generated from succinate dehydrogenase and then the generation of ROS. Complex II as the target of $\alpha$-TOS was further demonstrated by the observation that the ROS production and apoptosis process can only be triggered when complex II is functional in cells $^{207}$. Attractively, $\alpha$-TOS molecules exhibit specific selectivity towards cancerous cells rather than normal cells. This important selective property is supported by the fact that the ester structure in $\alpha$-TOS is responsible for its hydrolysis ${ }^{208}$. In normal cells, such hydrolysis reaction is enhanced but not in cancerous cells, resulting in that most of $\alpha$-TOS molecules will be hydrolyzed to form $\alpha-\mathrm{TOH}$ which is not supposed to occur in cancerous cells. Therefore, $\alpha$-TOS is quite promising in cancer treatment. $\alpha$-TOS is utilized as drug molecules that are delivered by MSNPs based delivery system which will be illustrated in following chapter with detail. Finally, the chemical structures of above mentioned mitochondria related anticancer agents were summarized. 


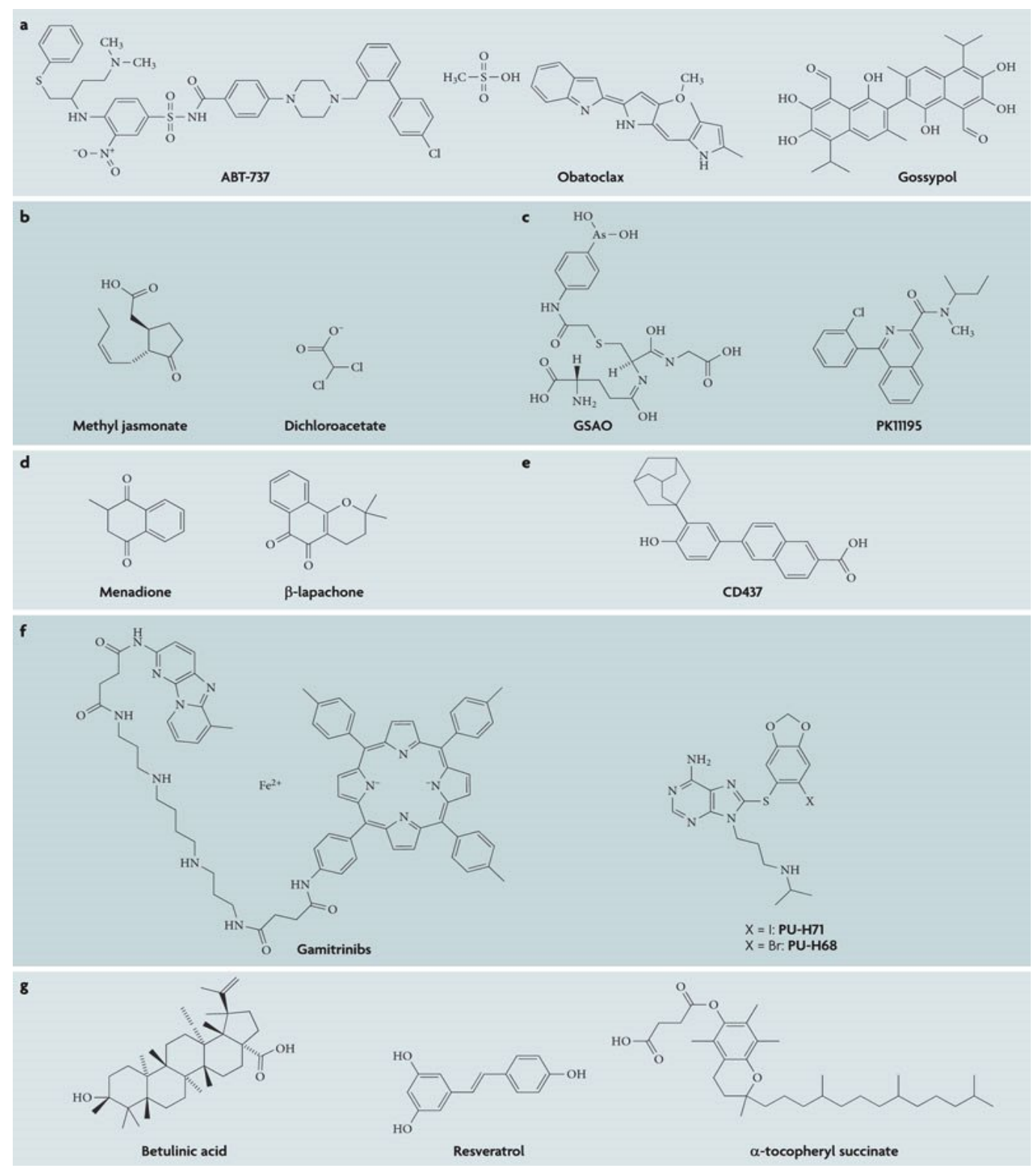

Figure 18. Summary of chemical structures of discussed mitochondria targeting anticancer agents. a. Modulators of the Bcl-2 protein family. b. Metabolic inhibitors. c. Agents targeting voltage-dependent anion channels. d.ROS generation regulators. e.Retinoids. f. Inhibitors of HSP 90. g. Natural compounds and derivatives.(Reproduced with permission from reference ${ }^{195}$, Copyright 2010 Rights Managed by Nature Publishing Group).

\subsection{Targeting mitochondria by nanoparticle system}

The research motivations and objectives of this thesis are based on the important role of mitochondria in cancer treatment, the advanced performance of MSNPs in drug delivery, and the very limited research in mitochondria targeting with drug delivery systems. To be specific, firstly, most of the investigations applying MSNPs as effective drug delivery system are 
conducted in cellular level. However, no published research regarding applications of MSNPs in sub-cellular drug delivery could be found before our investigation. The biological applications of MSNPs can be expanded by investigating MSNPs in sub-cellular drug delivery. Secondly, mitochondria have been demonstrated as an ideal target for cancer treatment, but very limited literatures with application of drug delivery systems in mitochondria targeting could be found. Such investigated drug delivery systems include polymer and liposome systems, without MSNPs. Therefore, it is rational to investigate the possible applications of MSNPs in cancer treatment with mitochondrial targeting ability. Thirdly, MSNPs have many advantageous properties over polymer and liposome systems such as easy synthesis and surface functionalization, versatility in cargo molecules, and commercial availability. In addition, MSNPs exhibit effectiveness in overcoming hydrophobicity of cargo molecules. For example, in Chapter 3, the anticancer application of alpha-TOS is hindered by its hydrophobicity. Such drawback can be overcome by MSNPs application and the effectiveness of alpha-TOS is enhanced.

The coherence of Chapter 2-4 is summarized as: the search in Chapter 2 demonstrated the rationality of applying MSNPs in mitochondrial drug delivery obtained primary data of anticancer effectiveness of DOX. With demonstrated rationality, considering that DOX is not mitochondrial specific, alpha-TOS and MSNPs with optimized size $(65 \mathrm{~nm})$ were selected and different cancer cell lines as well as normal cell line were investigated. Chapter 4 further investigated the expanding application of mitochondrial targeting MSNPs in overcoming DOX resistance based on data obtained in Chapter 2 (rationality of MNSPs application) and 3 (MSNPs with optimized size).

Up to date, very limited literature can be found in regard to the utilization of nanoparticle systems in targeting mitochondria. In these limited reports, the nanoparticle delivery systems mainly include liposomes, micelles and gold nanoparticles ${ }^{99,}{ }^{209}$. The overall designing 
mitochondrial targeted nanoparticle system shares the concept of incorporating anti-cancer drug molecules inside or on nanoparticles and mitochondrial targeting ligands are further conjugated $^{210}$. However, as one of the most effective drug delivery systems with various advantageous properties discussed before, MSNPs are not applied so far in targeted delivery of molecules to mitochondria. Compared with utilized nanoparticle based delivery system mentioned above, MSNPs possess great advantages such as good loading capacity, high stability, easy surface functionalization and biocompatibility. More importantly, MSNPs are quite easy to be fabricated in a relatively short period of time which makes MSNPs cost effective and commercially available. Therefore, during my PhD study, I mainly focused on fabrication of effective mitochondrial targeting delivery system based on MSNPs and the biological application of designed MSNPsas drug delivery system in cancer treatment including overcoming drug resistance.

\section{Chapter 2:Targeted Delivery of Doxorubicin to Mitochondria using Mesoporous Silica Nanoparticle Nano-carriers}

\subsection{Introduction}

Since the development of MSNPs, they have attached many interests applying MSNPs in targeted delivery of various drug molecules. As mentioned in chapter 1, MSNPs exhibit a number ofadvantages for effective drug delivery including controllable particle and pore size, easy synthesis, variable and large pore volume, easy surface functionalization, and biocompatibility. In the design of drug delivery system based on MSNPs, the commonly used strategies involve conjugating different chemical ligands with targeting ability to various receptor molecules on cell membrane. The receptor molecules of interest should be selected based on the differences between cancerous cell and normal cells. For example, the receptor 
molecules can be overexpressed on the cancer cell membrane but not in normal counterparts, resulting in different levels of such receptor between cancer cells and normal cells. The chemical ligands with targeting property should contain certain chemical structure that can be recognized by receptor on cell membrane. Such chemical ligands that have been utilized so far involve folic acid, RGD peptide as well as bioactive macro-molecules including biooligomers and various antibodies. In general, the design by conjugating targeting ligand on the surface of MSNPs is mainly for targeting cells in cellular level. For instance, through the conjugating of folic acid molecules on the surface of MSNPs, the functional MSNPs can be selectively internalized receptor mediated endocytosis process by target cells that have overexpressed folic acid receptor on cell membrane, leading to targeted delivery of drug molecules of interest. However, up to date, there are very limited designs on MSNPs to achieve the ability to target sub-cellular parts such as organelles to perform drug delivery within sub-cellular level. Therefore, here we are the first to investigate the suitability of MSNPs in targeting and delivering drug molecules to the important organelle, mitochondria. Mitochondria, known as the powerhouse of cells, are very important in the production of ATP molecules that is the commonenergy from used in cells. Mitochondria have been found to exhibit various characteristic features, including (1) Energy conversion and regulation of cellular metabolism. Apart from being the engine for ATP production, mitochondria are also acting as crucial cellular plant of metabolic substrates that are used for cell proliferation. This is quite important for cancerous cells because these cells need to maintain long lasting cell dividing which requires sufficient supply of metabolic substrates.(2) Reactive oxygen species (ROS) generation.The ROS level is crucial for normal cellular functions. High levels of intracellular ROS is very harmful since it can generate high levels of oxidative stress and trigger programmed cell death. Typically, ROS is generated along with various chemical reactions mainly occurring at the respiration chain within mitochondria. In order to maintain 
the balanced level of oxidative stress posed by ROS, cells have developed anti-oxidant system to neutralizethe oxidative stress.(3) Apoptosis-programmed cell death association. Mitochondria are consists of double lipid bilayers, the outer and inner mitochondrial membrane. Interestingly, the outer mitochondrial membrane is highly permeable whereas the inner mitochondrial membrane is highly non-permeable. What's more, due to the impermeability of inner membrane, mitochondrial matrix maintains high negative charge. It is reported that the cellular apoptosis can be triggered when mitochondria lose their integrity of inner membrane and the negative charge. The apoptotic hallmarks such as the release of cytochrome c, is observed when mitochondria lose their negative membrane potential.(4) Semi-autonomous organelle. It was reported that mitochondria DNA (mtDNA) encodes important proteins that areclosely related with ATP production, ROS generation, and aging. Another consideration that makes mitochondria suitable to be the target for cancer therapy is Warburg effect. As discussed before, in order to maintain the unlimited cell proliferation, much more energy and metabolic substrates are needed in cancer cells than those in normal cells in order to keep producing bioactive molecules such as proteins, lipids and nucleotides for rapid cell dividing. Paradoxically, it has been discovered for decades that the metabolic environment in cancerous cells lacks of oxygen consumption, andThe ATP production efficiency is much lower than that in normal cells because aerobic glycolysis stops with intermediates in cancer cells, but oxidative phosphorylation ends with $\mathrm{CO}_{2}$ and $\mathrm{H}_{2} \mathrm{O}$. The intermediates generated in cancer cells are critical in supply of substrates in cell proliferation.In addition, most metabolic reactions are occurring in mitochondria, leading to the conclusion that mitochondria are responsive for rapid proliferation in cancerous cells. Such unique phenomena make mitochondria in cancer cells more susceptible to mitochondria targeting therapeutics. Thus, mitochondria in cancerous cells may become the target of interest in cancer treatment. 


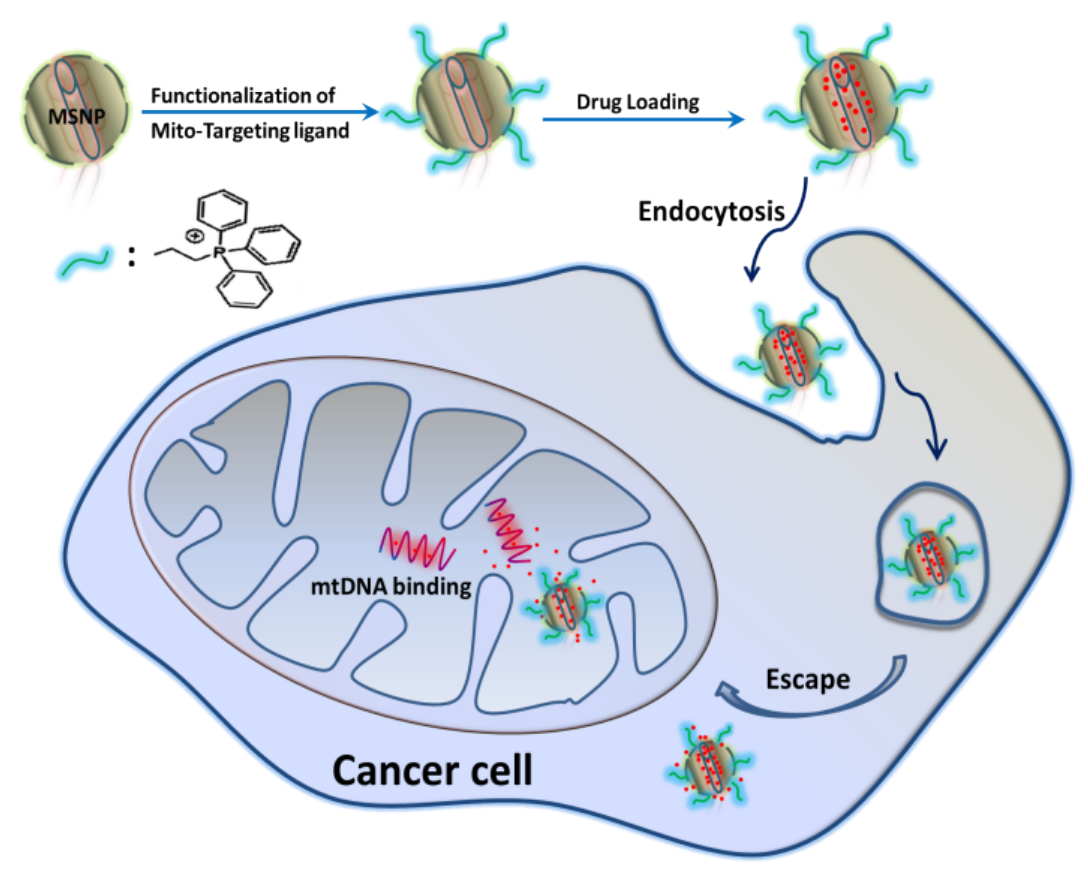

Figure 1. Schematic diagram of TPP conjugated MSNPs in mitochondrial sub-cellular drug delivery.(Reproduced with permission from reference ${ }^{210}$, Copyright 2015 Royal Society of Chemistry).

Here in our investigation, we applied TPP as effective mitochondrial targeting ligand which has been discussed before. To be specific, firstly, we have synthesized the well-ordered MSNPs with average diameter of $80 \mathrm{~nm}$. Owing to the property of easy surface functionalization of MSNPs, TPP ligand can be conjugated on the surface of MSNPs simply through one-step chemical reaction. The successful conjugation of TPP ligand on the surface of MSNPs was demonstrated by Fourier transform infrared (FT-IR) spectroscopy and Zeta potential detection studies. After that, various biological applications of designed TPP conjugated MSNPs were further investigated including cellular internalization, mitochondrial targeting effectiveness evaluation, anti-cancer cells proliferation evaluation as well as mitochondrial functions such as ATP production evaluation and mitochondrial membrane potential detection. Here we choose DOX as model anticancer drug. DOX anticancer drug has been extensively investigated as effective drug in wide range of cancer treatments. It is observed that DOX molecules can selectively interact with genetic DNA molecules by binding with big groove in DNA molecules, resulting in the dysfunctions of DNA molecules 
and further programmed cell death. It has been mentioned that mitochondria have their own genetic DNA molecules which can be reacted with DOX molecules. It is believed that, in our design, after TPP conjugation and DOX loading, MSNPs can selectively target and deliver DOX molecule into mitochondria, leading to mitochondrial malfunctions and apoptosis. Above all, the current research indicates the promising potential of applying MSNPs in subcellular drug delivery in a targeted way.

\subsection{Experimental materials and methods.}

\subsubsection{Applied materials and instruments}

Material: Absolute ethanol $(\mathrm{EtOH},>99.9 \%)$, sodium hydroxide $(\mathrm{NaOH}), 3-$ aminopropyltriethoxysilane (APTS), cetyltrimethylammonoium bromide (CTAB, 90\%), (4-carboxybutyl) triphenylphosphonium bromide (98\%), 4',6-diamidino-2phenylindole (DAPI), (3-(4,5-dimethylthiazol-2-yl)-2,5-diphenyltetrazolium bromide (MTT), doxorubicin (DOX), dulbecco's modified eagle's medium (DMEM), 1-ethyl3-(3-dimethylaminopropyl) carbodiimide (EDC), phosphate buffered saline (PBS), fetal bovine serum (FBS), fluorescein isothiocyanate (FITC), hydrochloride ( $\mathrm{HCl}$, 37\%), intracellular ATP determination kit (Invitrogen), Lyso-Tracker Green DND-26 (Invitrogen), mitochondria isolation kit for cultured cells (Thermo Scientific), MitoTracker Deep Red (Invitrogen), Mito-Tracker Green (Invitrogen), Nhydroxysulfosuccinimide (NHS), methanol (MeOH, 99.5\%), and tetraethylorthosilicate (TEOS, 99\%) were purchased commercially.Nanopure water (18.2 M $\Omega$, Millipore Co., USA) was used in all experiments and buffer preparations.

Instrument:Transmission electron microscopy (TEM) images were collected by using a JEOL JEL-1400 at $100 \mathrm{kV}$. Field emission electron microscopy (FE-SEM) images were obtained by using a FE-SEM 6340 (JEOL) at $5 \mathrm{kV}$. Specific surface areas and pore size 
distributions of the as-fabricated MSNPs were measured by using ASAP-2020 Mircomeritics. X-Ray diffraction patterns were collected by using aX'Pert Powder X-ray diffractometer. UV/Vis absorption intensities were recorded by using a UV/Vis 2501 Spectrometer. Zeta potentials were measured by using a MavernNanosizer. FT-IR spectra were measured by using a Fourier Transformed Infrared Spectrometer. A micro-plate reader (infinite 200 PRO, Tecan) was employed for the MTT assay, luminescence and fluorescence analysis. Confocal laser scanning microscopy images were taken by using a confocal microscope (Leica TCS SP5, 63× oil objective)

\subsubsection{Synthesis of MSNPs}

Typically, the synthesis of MSNPs was based on the conventional sol-gel process. Here the applied MSNPs were synthesized with amine surface functionalization and such modified MSNPs were fabricated based on the co-condensation method mentioned in chapter 1 . In general, sodium hydroxide solution with concentration of $2.0 \mathrm{M}$ was prepared at first. $500 \mathrm{mg}$ of CTAB compound was weighted and then dissolved in total volume of $240 \mathrm{~mL}$ deionized (DI) water. After totally dissolved in water to give the CTAB solution, one stir bar was added into the solution. After that, $1.3 \mathrm{~mL}$ of prepared sodium hydroxide solution was added into the CTAB solution. Then, one stir bar was added into the mixture solution and such mixture was equipped under vigorous stirring. At the same time, the stirring mixture solution was heated to $80{ }^{\circ} \mathrm{C}$. When the temperature of the mixture solution was stabilized at $80{ }^{\circ} \mathrm{C}$, the heated solution was then further maintained at $80{ }^{\circ} \mathrm{C}$ for $2 \mathrm{~h}$ under vigorous stirring. After that, $2.0 \mathrm{~mL}$ of silica precursor TEOS solution was added into the mixture solution dropwise. Keep the mixture solution under $80^{\circ} \mathrm{C}$ and vigorous stirring all the time when adding TEOS solution. Keep the solution for $15 \mathrm{~min}$, then $0.5 \mathrm{~mL}$ of APTES solution was added dropwise into the mixture solution. After adding TEOS and APTES solution, the newly formed mixture 
solution was further maintained at $80{ }^{\circ} \mathrm{C}$ and vigorous stirring for another $2 \mathrm{~h}$. Then, the white colored nanoparticles were formed and collected by centrifuging the obtained nanoparticle solution at $8000 \mathrm{rpm}$. The centrifugation process was conducted for three times washed with methanol and DI water. Finally, the obtained white nanoparticles were further dried under vacuum for $24 \mathrm{~h}$.

The removal of CTAB template molecules was conducted by using condensed $\mathrm{HCl}(37 \%)$ solution. Generally, the CTAB contained MSNPs were first suspended in $50 \mathrm{~mL}$ methanol solution. After that, the MSNPs suspended solution was equipped with heating to $80{ }^{\circ} \mathrm{C}$ and vigorous stirring. After the temperature of the mixture solution reached $80{ }^{\circ} \mathrm{C}, 3 \mathrm{~mL}$ of condensed $\mathrm{HCl}$ solution was added into the mixture solution. The new mixture solution was further heated under $80{ }^{\circ} \mathrm{C}$ and refluxed for $24 \mathrm{~h}$. Then, the MSNPs solution was centrifuged at $8000 \mathrm{rpm}$ for three times washed with methanol and DI water. Finally, MSNPs with empty pores were collected and dried under vacuum for $24 \mathrm{~h}$.

The TPP conjugated MSNPs was synthesized based on the chemical reaction between amine groups on the surface of MSNPs and the carboxylic groups on TPP molecules. It should be noted that the TPP conjugation process was conducted before CTAB removal in order to give MSNPs with TPP functionalization only on the outer surface. Typically, $50 \mathrm{mg}$ of TPP molecules were weighted and dissolved in $5 \mathrm{~mL}$ of DI water. After that, the TPP solution was further added and dissolved with $25 \mathrm{mg}$ EDC and $35 \mathrm{mg}$ NHS which aims at activating the carboxylic groups on TPP. The formed mixture solution was stirred at dark for $2 \mathrm{~h} .10 \mathrm{mg}$ of obtained MSNPs- $\mathrm{NH}_{2}$ was weighted and suspended in $10 \mathrm{~mL}$ DI water. After that, such nanoparticles suspended solution was added with the pre-activated TPP solution. Then the new mixture solution was put under stirring for $48 \mathrm{~h}$. The nanoparticles were then collected by centrifugation at $8000 \mathrm{rpm}$ for three times washed with methanol and DI water. The 
removal of CTAB template was conducted as mentioned to form MSNPs with outer surface conjugation of TPP which are mark as MSNPs-PPh ${ }_{3}$.

In order to trace the location within cellular level, fluorescent MSNPs were also fabricated by conjugating FITC on the surface. Typically, $1.3 \mathrm{mg}$ of FITC was weighted and dissolved in $1.0 \mathrm{~mL}$ of absolute ethanol solution. After that, $3 \mu \mathrm{L}$ of APTES was added into FITC solution to form new mixture solution and such mixture solution was placed at dark for $2 \mathrm{~h}$. The prepared FITC/APTES solution was added after APTES addition described above to form the yellow colored nanoparticles. Such nanoparticles were collected by centrifugation for three time washed with methanol and DI water.

\subsubsection{DOX loading and releasing}

Firstly, DOX solution with concentration of $1 \mathrm{mg} / \mathrm{mL}$ was prepared as DOX stock solution. The DOX loading process was conducted by suspending $1.0 \mathrm{mg}$ MSNPs in $1.0 \mathrm{~mL}$ DOX stock solution. Then the MSNPs contained DOX stock solution was placed at dark under stirring for $24 \mathrm{~h}$. After that, MSNPs were loaded with DOX molecules due to the diffusion process driven by concentration difference of DOX. The DOX loaded MSNPs were then collected by centrifugation at $10000 \mathrm{rpm}$ for $5 \mathrm{~min}$. Then, the obtained DOX loaded MSNPs were further suspended in DI water and re-centrifuged at $10000 \mathrm{rpm}$ for $5 \mathrm{~min}$. The resuspending process was repeated for three time in order to eliminate the DOX molecules on the surface of MSNPs. The DOX loading capacity of MSNPs was calculated based on the difference of UV-vis intensities at $485 \mathrm{~nm}$ between DOX solution after MSNPs loading and the original DOX stock solution. The DOX loading process was repeated for three time.

As for the in vitroDOX releasing of DOX loaded MSNPs, $500 \mathrm{mg}$ of DOX loaded MSNPs were weighted and suspended in $2.0 \mathrm{~mL}$ PBS solution. After that, the nanoparticles suspended PBS solution was placed under stirring for different periods of timesfor example 4 
h, 8 h, 12 h, 24 h, 48 h, 36 h and 72 h. At each time point, the MSNPs contained PBS solution was centrifuged and the supernatant was collected. Then the UV-vis intensities at $485 \mathrm{~nm}$ of each collected supernatant were obtained. The DOX concentrations of each supernatant were calculated based on the obtained UV-vis intensities. Finally, the DOX leasing test was repeated for three times.

\subsubsection{Cell culture}

HeLa cancerous cells were cultured in DMEM containing 1\% penicillin/streptomycin (Invitrogen) and $10 \%$ FBS (Invitrogen). The cells were cultured in incubator which has condition of maintained humidified atmosphere containing $5 \% \mathrm{CO}_{2}$ under $37{ }^{\circ} \mathrm{C}$.

\subsubsection{Cytotoxicity assay (MTT)}

In general, HeLa cells were cultured in good condition with good cellular status. HeLa cells were then seeded in a 96-well plate with cell density of $1 \times 10^{4}$ per well with $200 \mu \mathrm{L}$ culturing medium. The seeded cells were cultured for $24 \mathrm{~h}$. After that, the old culturing medium was removed in each well and new culturing medium of $200 \mu \mathrm{L}$ containing different concentration of MSNPs was added in each well. The cells were then cultured for another 24 h. After that, the old culturing medium was removed in each well. New culturing medium of $100 \mu \mathrm{L}$ containing MTT at concentration of $0.5 \mathrm{mg} / \mathrm{mL}$ was added into each well. Then the 96-well plate was placed at culturing condition for $4 \mathrm{~h}$. Then, the old medium was removed in each well and $100 \mu \mathrm{L}$ DMSO solution was added into each well. After that, the plate was gently shacked for $5 \mathrm{~min}$. Then the absorbance intensities of wells at $560 \mathrm{~nm}$ were collected by using micro plate reader. The cell survival rate was calculated based on the different absorbance intensities between experimental wells and control wells. Each concentration of MSNPs was evaluated for 6 times. 


\subsubsection{Confocal laser microscopy study}

In general, HeLa cells were cultured in good condition with good cellular status. One 6-well plate was filled with cover sides in each well at first. Hela cells were then seeded in the 6well plant at a cell density of $1 \times 10^{5}$ per well and $2.0 \mathrm{~mL}$ of culturing medium was added in each well. The cell seeded plate was cultured for $24 \mathrm{~h}$ in incubator. After that, the old culturing medium was removed and new culturing medium of $2.0 \mathrm{~mL}$ with different MSNPs sample with FITC conjugation or DOX loading inside was added into each well. Then, the plate was further placed under culturing condition for another $24 \mathrm{~h}$. For nucleus staining, the old medium was removed and cells were fixed by adding $1.0 \mathrm{~mL}$ of $4.0 \%$ formaldehyde solution in each well. After that, fixing solution was removed and cells were washed with 1.0 $\mathrm{mL}$ PBS solution in each well for three times. Then cells were treated with new medium containing DAPI staining solution with concentration of $30 \mathrm{nM}$. After culturing cells for another $15 \mathrm{~min}$, staining solution was removed in each well and cells were further washed with PBS for three times. Then the cells on cover slide were sealed with nail oil to be the sample for confocal laser microscopy. As for mitochondria staining, the old medium was removed and new medium of $1.0 \mathrm{~mL}$ containing mitochondrial staining solution (MitoTracker red and green) with concentration of $50 \mathrm{nM}$ was added into each well. The cells were culture with staining solution for $45 \mathrm{~min}$. After that, the old medium was removed and cells were washed with $1.0 \mathrm{~mL}$ PBS solution for three times. Then cells were treated with $1.0 \mathrm{~mL}$ $4.0 \%$ formaldehyde solution in each well for being fixed. After that, cells on the cover slide were sealed with nail oil to be the sample for confocal laser microscopy. In regards to lysosome staining, HeLa cells were seeded in plastic-bot-tomedm-dishes $(35 \mathrm{~mm})$ at cell density of $1 \times 10^{5}$ and cultured in complete DMEM for $24 \mathrm{~h}$. After that, the old medium was removed and new culturing medium containing different MSNPs samples were added in each dish. Then dishes were cultured with MSNPs for different periods of times including $4 \mathrm{~h}, 8 \mathrm{~h}$ 
and $12 \mathrm{~h}$. After such incubating, the old medium was removed and new culturing medium was added and cells were directly observed under confocal laser microscopy without any washing process.

The fluorescence of Lyso-Tracker Green and DAPI was excited at $\lambda=488 \mathrm{~nm}$ and $\lambda=405$ nm, respectively. The fluorescence of Mito-Tracker Deep Green and Red was excited at $\lambda=$ $488 \mathrm{~nm}$ and $\lambda=594 \mathrm{~nm}$, respectively. The fluorescence signals of FITC and DOX were collected by being excited by laser with wavelength of $488 \mathrm{~nm}$. The fluorescence of DAPI, FITC, Mito-Tracker Deep Red, Mito-Tracker Green, Lyso-Tracker Green and DOX was collected at $\lambda=(460 \pm 10),(520 \pm 10),(620 \pm 10),(520 \pm 10),(530 \pm 10)$ and $(580 \pm 10) \mathrm{nm}$ channels, respectively. All fluorescence channels were detected sequentially.

\subsubsection{Mitochondria isolation}

The isolation of mitochondria from HeLa cells was conducted on the basis of a commercially available kit provided by Thermo Scientific. Typically, HeLa cells were cultured in T-75 cell culture flask at a cell density of $1 \times 10^{7}$ with good cellular status. Then cells were treated with trypsin in order to harvest cells. After trypsin treatment, cells were collected by centrifugation at $850 \times \mathrm{g}$ for $2 \mathrm{~min}$ in a $2.0 \mathrm{~mL}$ centrifuging tube. Cells were pelleted in the tube. Then, pelleted cells were treated with $800 \mu \mathrm{L}$ of rupturing agent provided by the mitochondria isolation kit followed by putting tube with cells under vortex for 5 seconds. Then the cell contained tube was further placed on ice for $2 \mathrm{~min}$. After that, the cell contained tube was treated with $10 \mu \mathrm{L}$ mitochondria isolation reagent provided by the kit. Then the tube was put under vortex for 5 seconds followed by placing the tube on ice for 5 min. After incubation on ice, the tube containing cells was further treated with 800 $\mu$ Lmitochondria isolation reagent and being converted for several times. After that, the tube containing cells was centrifuged at $700 \times \mathrm{g}$ for $10 \mathrm{~min}$ in environment of $4{ }^{\circ} \mathrm{C}$. The 
supernatant was collected and transferred to a new $2.0 \mathrm{~mL}$ centrifuging tube. Then the tube containing supernatant was further centrifuged at $12000 \times \mathrm{g}$ for $15 \mathrm{~min}$ in environment of $4{ }^{\circ} \mathrm{C}$. Finally, the isolated mitochondria were collected as pellet. After that, the tube containing mitochondria pellet was added with $200 \mu \mathrm{L}$ PBS solution to suspend the collected mitochondria. And the suspended mitochondria solution was transferred to 96-well plate and its fluorescent intensity excited by $488 \mathrm{~nm}$ was detected by using micro plate reader.

\subsubsection{Intracellular ATP production level detection}

The intracellular ATP production level in HeLa cells was detected by applying the ATP determination kit provided by Invitrogen. The procedures were conducted based on the protocol by Invitrogen. Briefly, HeLa cells were cultured in good condition with good cellular status. Then HeLa cells were seeded in a 6 -well plate at a cell density of $1 \times 10^{5}$ per well. The seeded cells were cultured under good condition for $24 \mathrm{~h}$. After that, the old medium was removed and new culturing medium containing different MSNPs samples was added into each well. The cells were further incubated for another $24 \mathrm{~h}$. Then cells in the plate were washed with $1.0 \mathrm{~mL}$ PBS solution for three times and treated with $200 \mu \mathrm{L}$ cell lysis buffer (Invitrogen). Then cellular structure was destroyed to form the cellular debris. Such debris were collected as ATP containing experimental sample to be tested for ATP level. The debris were then treated with reacting agent provided by Invitrogen to exhibit fluorescent intensity. The quantification of intracellular ATP level was conducted based on comparing the fluorescent intensity with the standard curve obtained by calculating the fluorescent intensities generated by a series of standard ATP solutions.

\subsubsection{Intracellular mitochondria membrane potential detection}

The intracellular mitochondria membrane potential was detected in HeLa cells by applying the detection kit provided by Abcam. The measuring procedures were based on the standard 
protocol by Abcam. Basically, HeLa cells were cultured in good condition with good cellular status. Then HeLa cells were seeded in a 96-well plate at a cell density of $1 \times 10^{4}$ per well. Then cells were cultured in complete DMEM medium for $24 \mathrm{~h}$. After that, the old culturing medium was removed and $200 \mu \mathrm{L}$ new culturing medium containing different MSNPs samples was added into each well. The cells were further cultured with MSNPs samples for another $24 \mathrm{~h}$. After that, the old medium was removed and cells were washed with PBS solution for two times followed by adding new culturing medium with tetramethylrhodamine ethyl ester (TMRE) in final concentration of $200 \mathrm{~nm}$. Then the treated cells were cultured for 20 min under incubating condition. After that, the incubated Hela cells were washed with PBS solution for one time and the fluorescent intensities of TMRE of wells were detected at $549 \mathrm{~nm}$ by using the micro plate reader.

\subsubsection{Flow cytometry analysis}

HeLa cells were cultured in good condition with good cellular status. For sample preparation for flow cytometry analysis, HeLa cells were seeded in a 6-well plate at a cell density of $1 \times 10^{5}$ per well with $2.0 \mathrm{~mL}$ culturing medium and incubated under good condition for $24 \mathrm{~h}$. After that, the old culturing medium was removed and new culturing medium of 2.0 $\mathrm{mL}$ containing different MSNPs samples was added into each well. Then cells were further cultured for another $24 \mathrm{~h}$. After that, the old medium was removed and cells were washed with PBS solution for two times followed by adding new culturing medium with tetramethylrhodamine ethyl ester (TMRE) in final concentration of $200 \mathrm{~nm}$. After treating cells under culturing condition for $20 \mathrm{~min}$, the old medium was removed and the cells were washed with $1.0 \mathrm{~mL}$ PBS solution for three times. Then the cells were harvested by being treated with trypsin. Finally, cells were collected by being centrifuged at $3000 \mathrm{rpm}$ for $5 \mathrm{~min}$ and were suspended in PBS solution for flow cytometry analysis. 


\subsection{Results and discussion}

\subsubsection{Fabrication and characterizations of MSNPs}

The synthesized MSNPs were directly visualized and characterized by applying and field emission scanning electron microscopy (FE-SEM) and transmission electron microscopy (TEM) (Figure 2). Through calculating the diameters of 50 single nanoparticles in obtained TEM images, the average diameter of fabricated MSNPs was around $80 \mathrm{~nm}$, which is supposed to be suitable for biological application. In addition, the mesoporous structure of MSNPs can be primarily visualized in the obtained TEM images. In order to further characterize the size of synthesized MSNPs, dynamic light scattering (DLS) analysis was also conducted. The obtained size of MSNPs was $148 \mathrm{~nm}$ according to DLS study, which was a little bit larger than that obtained for TEM images.

The nanoparticle size detected by DLS was conducted in DMEM cell culture medium. The obtained larger nanoparticle size may be resulted from the interactions between mitochondrial targeting ligand $\left(-\mathrm{PPh}_{3}\right)$ functionalization on surface of MSNPs and substances in DMEM such as amino acids and glucose. Detecting DLS nanoparticles size in DEME is to simulate the actual cellular treatment of MSNPs. 

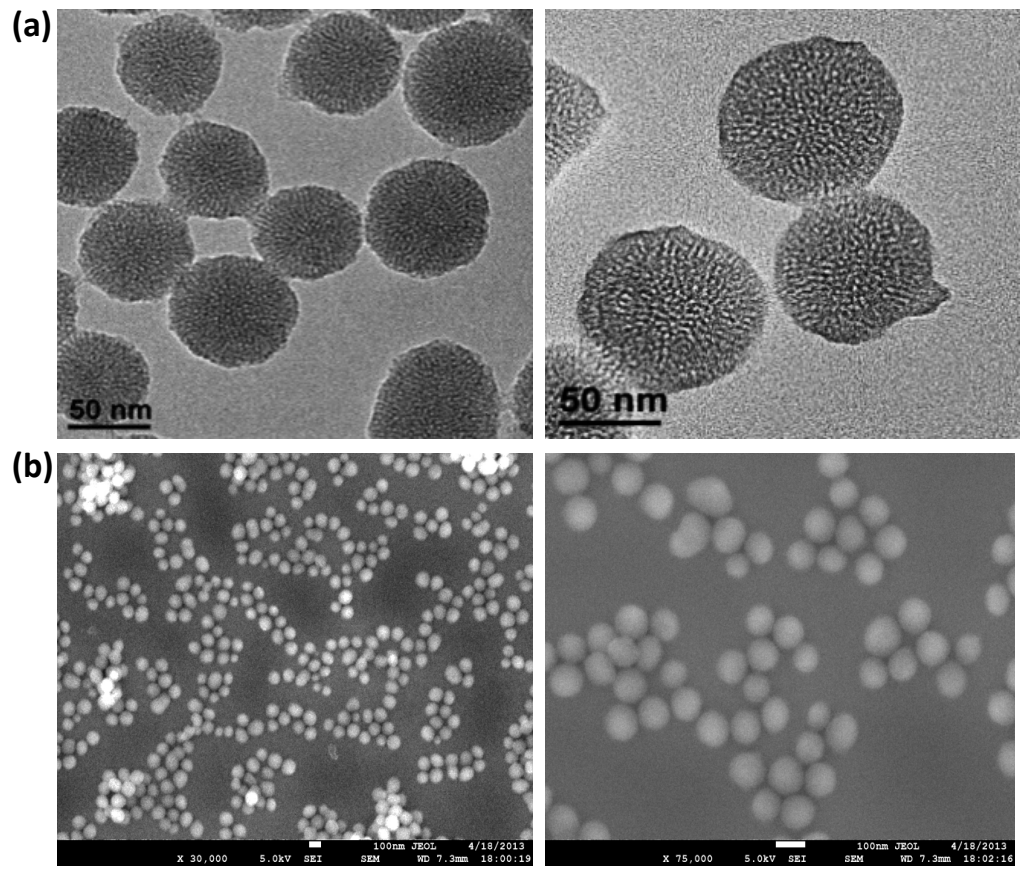

Figure 2. TEM (a) and FE-SEM (b) images of obtained MSNPs-PPh ${ }_{3}$. Scale bar in FE-SEM images is $100 \mathrm{~nm}$. (Reproduced with permission from reference ${ }^{210}$, Copyright 2015 Royal Society of Chemistry).

The preparation methodology of MSNPs was based on surfactant directed sol-gel reaction. The surfactant molecule utilized is CTAB. Here in our case, the functionalization of amine groups on the surface of MSNPs was achieved by co-condensation method. To be specific, co-condensation means that during the interaction between surfactant directed micelles and silica precursors, the silica precursors involve more than one kind of molecules. For example, TEOS is the most commonly applied silica precursor and in our fabrication of $\mathrm{MSNPs}-\mathrm{NH}_{2}$, another silica precursor, APTES, was also applied in order to give amine functionalization. It should be noticed that amine functionalization by co-condensation method results in functionalizing both on outer surface and the inner surface of MSNPs. The selective surface functionalization of MSNPs can be achieved by another method called post-synthesis grafting which has been discussed before. 

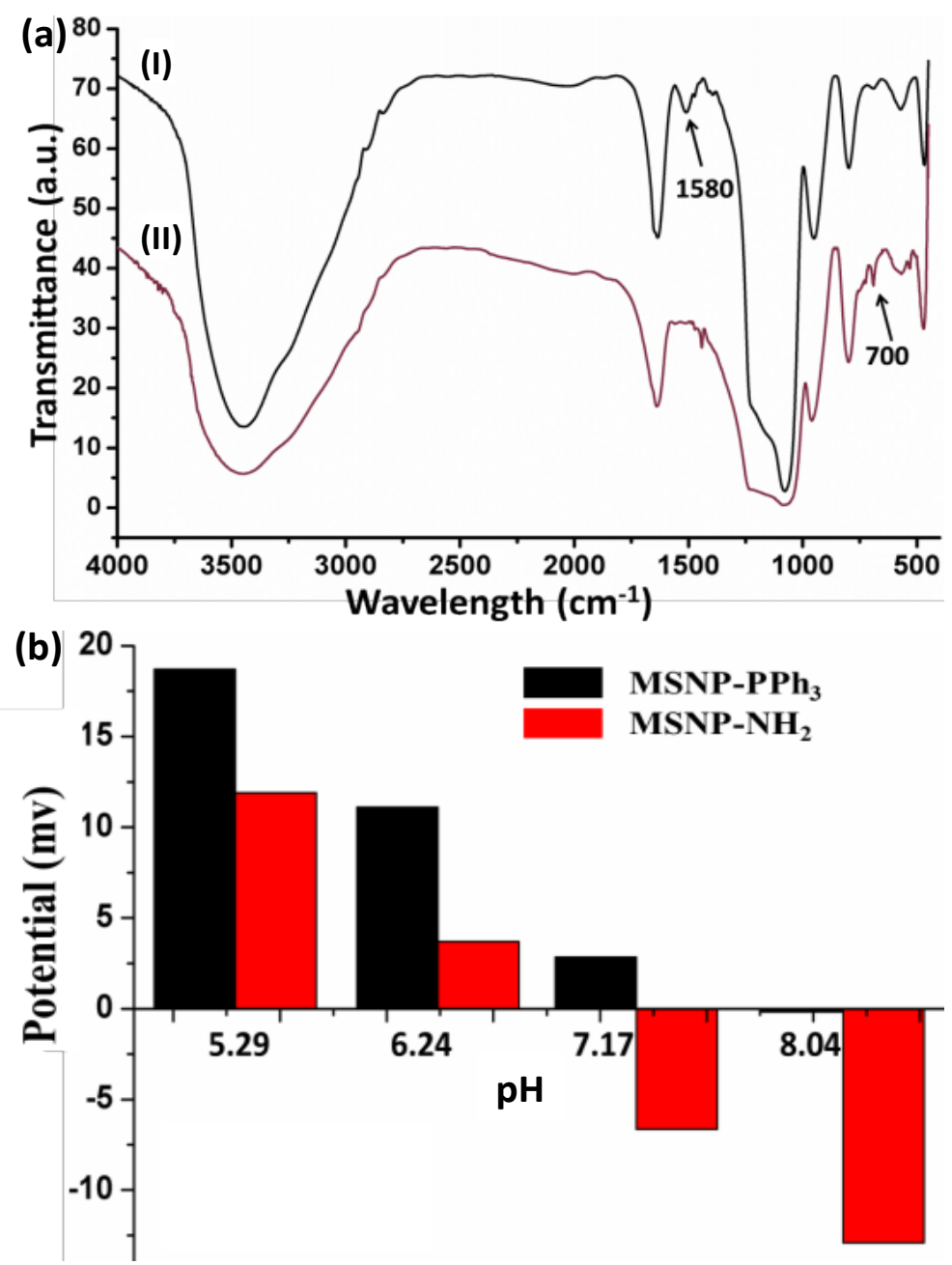

Figure 3.(a) FT-IR spectra of MSNPs-NH $\mathrm{NH}_{2}$ (I) and $\mathrm{MSNPs}-\mathrm{PPh}_{3}$ (II).(b) Zeta potential results of MSNPs-NH $\mathrm{N}_{2}$ and MSNPs-PPh ${ }_{3}$ suspended in PBS with different $\mathrm{pH}$ values. (Reproduced with permission from reference ${ }^{210}$, Copyright 2015 Royal Society of Chemistry).

The successful amine functionalization was demonstrated by using FTIR and Zeta potential detection methods. As shown in the FTIR result image (Figure 3a), in curve I, one obvious peak around $1580 \mathrm{~cm}^{-1}$ was presented, which is corresponding to the amine groups on the surface of MSNPs-NH . Such peak clearly indicated the successful functionalization of amine groups on MSNPs. However, in the case of curve II, there was no clear peak presenting at around $1580 \mathrm{~cm}^{-1}$, meaning that the amount of amine groups on the surface of MSNPs$\mathrm{PPh}_{3}$ was significantly decreased. Considering that the TPP conjugation is based on the chemical reaction between amine groups on the nanoparticle surface and carboxylic groups on TPP compounds, the decrease of amine groups on the surface of MSNPs- $\mathrm{PPh}_{3}$ was caused 
by TPP conjugation. Hence, the successful conjugation of TPP on the surface of MSNPs was demonstrated as well. Additionally, it is observed that another peak at around $700 \mathrm{~cm}^{-}$ ${ }^{1}$ appeared in curve II, which should be ascribe to the deformation vibration of carbonhydrogen bonds in phenyl groups, indicating the presence of phenyl groups on the surface of MSNPs-PPh $h_{3}$. Such observation also confirmed the successful formation of MSNPs-PPh ${ }_{3}$.

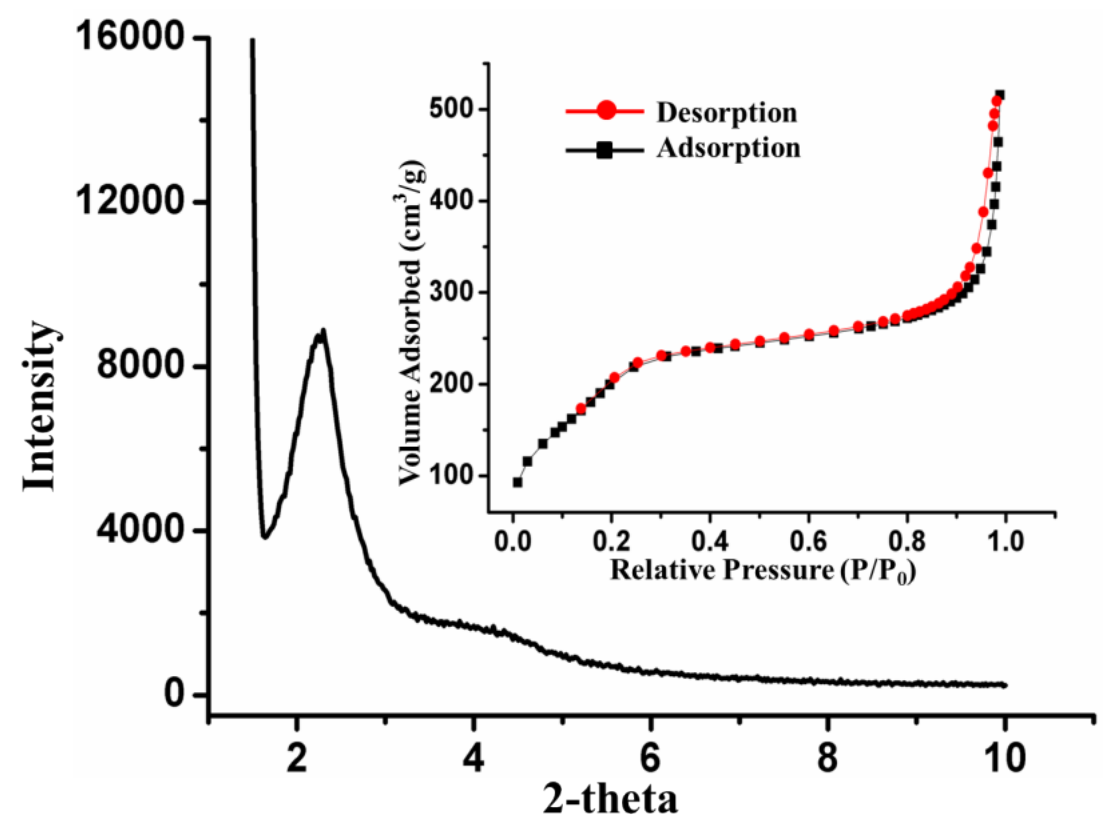

Figure 4.Powder XRD pattern and BET isotherm curve (insert) of $\mathrm{MSNPs}_{\mathrm{PPh}}$. (Reproduced with permission from reference ${ }^{210}$, Copyright 2015 Royal Society of Chemistry).

Moreover, in order to further characterize the conjugation of TPP on the surface, Zeta potential values of MSNPs-PPh 3 and MSNPs-NH $\mathrm{NH}_{2}$ at different $\mathrm{pH}$ values of PBS solutions were also investigated (Figure 3b). The design is based on the fact that due to different deprotonation/protonation environments, $-\mathrm{NH}_{2}$ functionality shows negative charges under basic conditions and positive surface charges under acidic conditions. This can be observed from the results by detecting the surface charges of MSNPs- $\mathrm{NH}_{2}$ under basic and acidic conditions. However, in the case of detecting the surface charges of MSNPs- $\mathrm{PPh}_{3}$, the surface charges showed positive in acidic conditions whereas still positive or not negative in basic conditions, indicating that the amine groups on the surface of $\mathrm{MSNPs}-\mathrm{PPh}_{3}$ was significantly 
lower than that on MSNPs- $\mathrm{NH}_{2}$. In addition, with consideration of TPP functionalization reaction, the successful TPP conjugation on MSNPs was further demonstrated.

The Brunauer-Emmett-Teller (BET) surface area and Barrett-Joyner-Halenda (BJH) pore size of MSNPs- $\mathrm{PPh}_{3}$ was characterized by applying $\mathrm{N}_{2}$ adsorption/desorption measurement (Figure 4). The obtained values were $739.699 \mathrm{~m}^{2} / \mathrm{g}$ and $2.07 \mathrm{~nm}$ for BET surface area and $\mathrm{BJH}$ pore diameter, respectively. In addition, the successful formation of mesoporous structure corresponding to MCM-41 type of MSNPs was determined by the observation of type IV BET isotherm curve. Through powder X-Ray diffraction (XRD) analysis, the wellordered hexagonal structure of $\mathrm{MSNP}-\mathrm{PPh}_{3}$ was demonstrated based on the appearance of (100) peak in the result.

\subsubsection{DOX loading, release and mitochondria targeting}

DOX was utilized in our system as model anticancer drug because it is extensively investigated that DOX molecules can interact with DNA genetic molecules and affect the function of nucleus. It is proposed that DNA molecules in mitochondria (mtDNA) are susceptible to DOX as well. Therefore, DOX can be selected as mitochondrial affecting drug molecules. DOX molecules need to be loaded into the meso-pores of MSNPs at first. It is found that DOX molecules can be easily diffused into MSNPs driven by the concentration difference between the DOX loading solution and the microenvironment inside the mesopores. The resulting loading capacities of DOX for $\mathrm{MSNPs}-\mathrm{PPh}_{3}$ and $\mathrm{MSNPs}-\mathrm{NH}_{2}$ were 3.05 $w t \%$ and $2.95 w t \%$, respectively. The loading capacity of MSNPs was estimated by comparing the different VU-vis intensities between original DOX loading solution and DOX solution after drug loading. The actual amount of DOX in solution was calculated based on standard curve of standard DOX solutions with various concentrations. 


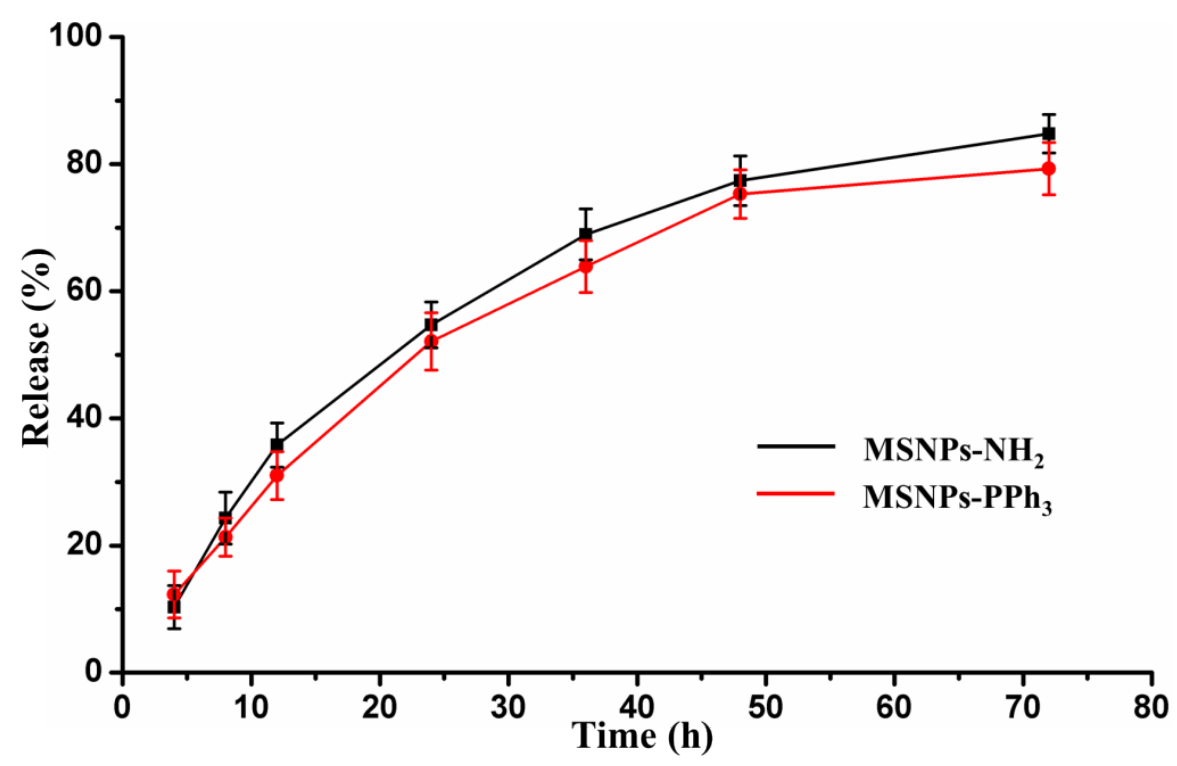

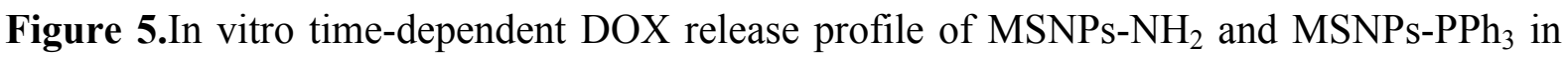
PBS solution $(\mathrm{PH}=7.0)$. (Reproduced with permission from reference ${ }^{210}$, Copyright 2015 Royal Society of Chemistry).

The DOX release process means the diffusion of DOX molecules from inside meso-pores to outside. The driving force for DOX releasing is the concentration difference between the microenvironment inside meso-pores and the outside microenvironments such as cytoplasm and mitochondrial matrix. The in vitro releasing profiles of DOX from $\mathrm{MSNPs}-\mathrm{PPh}_{3}$ and MSNPs- $\mathrm{NH}_{2}$ were investigated (Figure 5).

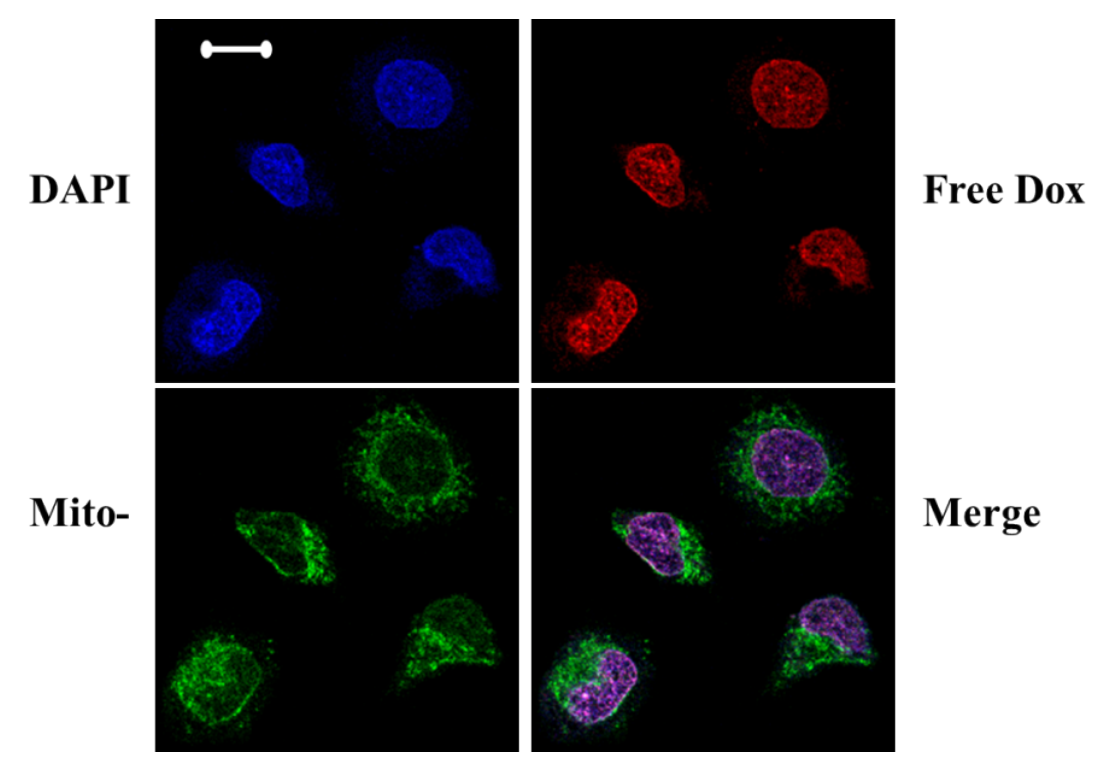

Figure 6.Confocal microscope images of HeLa cells after being treated with free DOX $(4 \mu \mathrm{g} / \mathrm{mL})$ for $6 \mathrm{~h}$. Mitochondria were stained by Mito-Tracker Green $(50 \mathrm{nM})$. Scar bar: 20 
$\mu \mathrm{m}$.(Reproduced with permission from reference ${ }^{210}$, Copyright 2015 Royal Society of Chemistry).

In the study of mitochondria targeting, we first investigated the intracellular interaction between DOX molecules and mitochondria. From the resulting confocal microscopy images (Figure 6), we can observe that free DOX molecules would directly and selectively accumulate in the nucleus, which was indicated by the merged purple color resulted from the red color presenting the DOX molecules and blue color representing the nucleus DNA molecules. Thus, it can be concluded that free DOX molecules do not have any interaction with mitochondria.
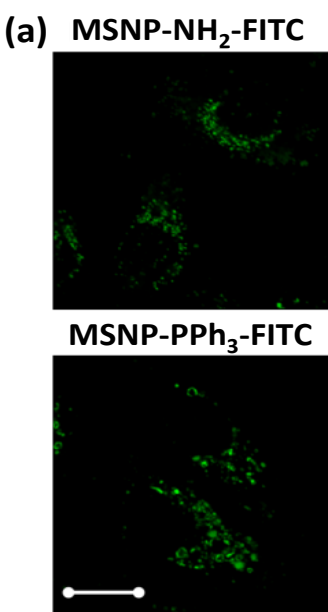

(b) MSNP-NH - -DOX

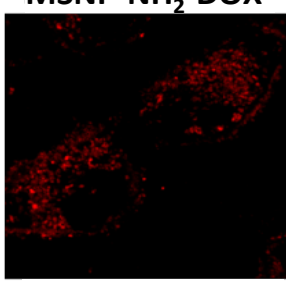

MSNP-PPh ${ }_{3}-\mathrm{DOX}$

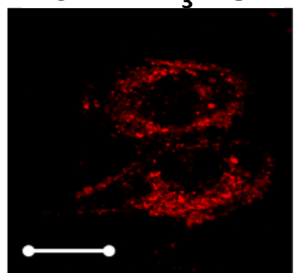

Mito-

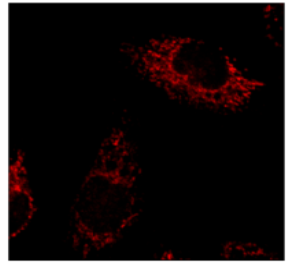

Mito-

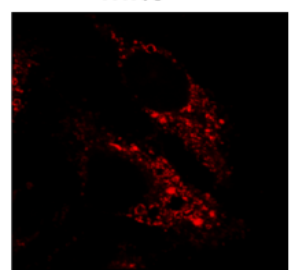

Mito-

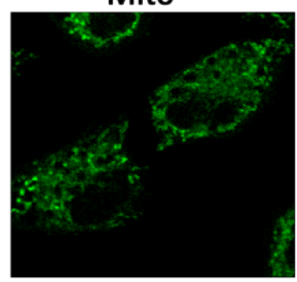

Mito-

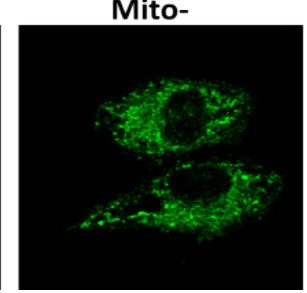

Merge

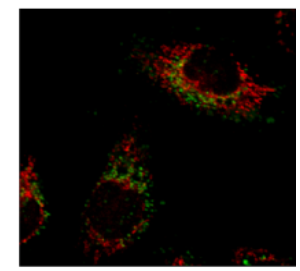

Merge

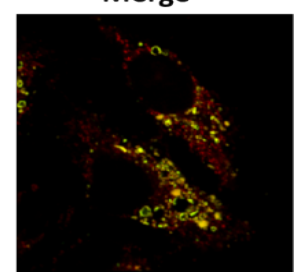

Merge

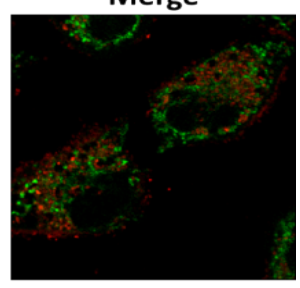

Merge

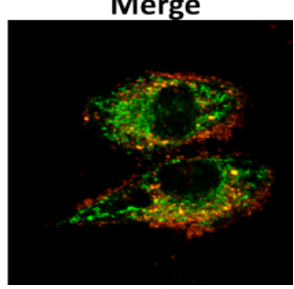

Figure 7.(a) Confocal microscope results of Hela cells after being treated with $\mathrm{MSNPs}-\mathrm{PPh}_{3}$ FITC and MSNPs-NH ${ }_{2}$-FITC $(50 \mu \mathrm{g} / \mathrm{mL})$. Mitochondria were stained by Mito-Tracker Red $(50 \mathrm{nM})$. (b) Confocal microscope results of Hela cells after being treated withMSNPs-PPh $\mathrm{Ph}_{3}$ DOXand MSNPs-NH $\mathrm{N}_{2}$-DOX $(50 \mu \mathrm{g} / \mathrm{mL})$. Mitochondria were stained by Mito-Tracker Green $(50 \mathrm{nM})$. Scar bar: $20 \mu \mathrm{m}$. (Reproduced with permission from reference ${ }^{210}$, Copyright 2015 Royal Society of Chemistry). 
Next, we began to investigate the mitochondria targeting effect of $\mathrm{MSNPs}-\mathrm{PPh}_{3}$ and MSNPs$\mathrm{NH}_{2}$ in HeLa cancerous cells since mitochondria inside cancerous cells emerged as potential target for cancer treatment mentioned before. We incubated HeLa cells with $\mathrm{MSNPs}-\mathrm{PPh}_{3}$ and MSNPs- $\mathrm{NH}_{2}$ with FITC functionality or DOX loading, then confocal microscopy images were obtained for analyzing the interactions between mitochondria and MSNPs. It is shown in the resulted mages that (Figure 7), firstly, MSNPs could be well internalized by HeLa cells for both TPP and amine conjugation, which means that MNSPs showed good biocompatibility in terms of cellular uptake. In addition, from the images, the mitochondria inside HeLa cells could be well stained as shown in red or green color, indicating that mitochondria in HeLa cells were quite active and exhibited active membrane potential because the successful staining of mitochondria relied on the active mitochondrial membrane potential. Secondly, the resulting images showed effective targeting of $\mathrm{MSNPs} \mathrm{PPh}_{3}$ to mitochondria in HeLa cells. This result could be supported by the merged images in the cases of sampling with both MSNPs-PPh ${ }_{3}$-FITC and MSNPs-PPh ${ }_{3}$-DOX. To be specific, in figure7a, the green color was representing the fluorescent signal from FITC which was used for MSNPs visualization. And the stained mitochondria were appeared as red color from Mito-Tracker Red. From the merge images, no obvious merged color (yellow) was found when sampling with MSNPs-NH 2 -FITC. However, in the case of applying $\mathrm{MSNPs}-\mathrm{PPh}_{3}$ FITC, clear yellow color was observed in the merged image, indicating the co-coloration of green and red color. In other words, mitochondria in HeLa cells were co-localized with MSNPs-PPh ${ }_{3}$-FITC but not with MSNPs-NH ${ }_{2}$-FITC. When MSNPs were loaded with DOX molecules (MSNPs-PPh $-\mathrm{DOX}$ and MSNPs-NH $2-\mathrm{DOX}$ ), MSNPs could be visualized as red color due to the red fluorescence signal from DOX molecules, which is just like staining MSNPs with DOX. From figure 7b, similarly, no significant yellow color could be observed when cells were treated with MSNPs- $\mathrm{NH}_{2}-\mathrm{DOX}$, meaning that no co-localization effect 
occurred between MSNPs- $\mathrm{NH}_{2}$-DOX and mitochondria in HeLa cells. Whereas, in the case of sampling with MSNPs- $\mathrm{PPh}_{3}$-DOX, the merged yellow color could be found, indicating the co-localization of MSNPs-PPh 3 -DOX and mitochondria in HeLa cells. What's more, the colocalization efficient values of all merged images were calculated in Image J software. In figure $7 \mathrm{a}$, such values were calculated as 0.749 versus 0.278 and 0.758 versus 0.360 for Pearson's Coefficient and Overlap Coefficient, respectively. Similarly, figure $7 \mathrm{~b}$, such values were calculated as 0.593 versus 0.225 and 0.570 versus 0.289 , respectively. Higher values of Pearson's Coefficient and Overlap Coefficient represent better co-localization effect. Finally, we can conclude that TPP functionality on the surface of MSNPs exhibits effective facilitation on targeting mitochondria in HeLa cells. With the consideration to make confocal microscopy results more convincing, all the images were obtained by sequentially adjusting different laser channels and powers.

\subsubsection{Mitochondria isolation for fluorescence analysis}

Apart from utilizing confocal laser microscopy in mitochondria targeting effect of MSNPs, we also introduced the method of fluorescence analysis of isolated mitochondria to investigate the existence of MSNPs in mitochondria. Here we applied MSNPs with fluorescence signal (MSNPs-PPh 3 -DOX and MSNPs-NH $\mathrm{N}_{2}-\mathrm{DOX}, \mathrm{MSNPs}-\mathrm{PPh}_{3}-\mathrm{FITC}$ and MSNPs-NH 2 -FITC). After treated with fluorescent MSNPs, mitochondria in HeLa cells were isolated for fluorescence analysis. MSNPs- $\mathrm{PPh}_{3}-\mathrm{DOX}$ and MSNPs-PPh 3 -FITC were set as test groups and MSNPs without TPP functionality or fluorescence signal were set as control groups. It should be noted that in order to eliminate the affection by free MSNPs, the isolated mitochondria were washed with PBS solution for three times, resulting in more convincible data. 


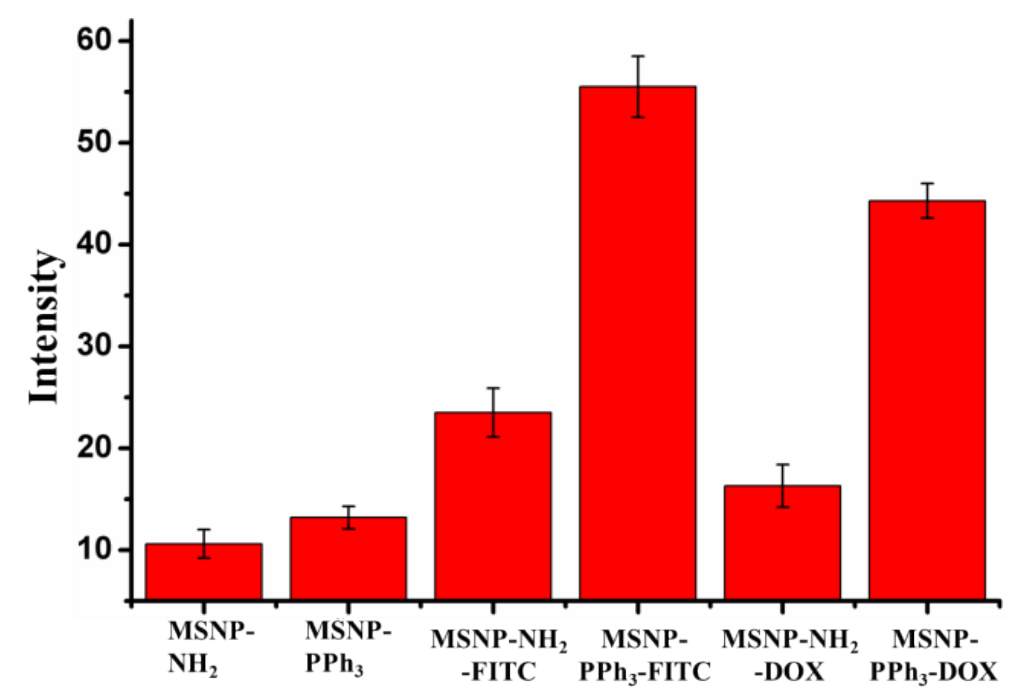

Figure 8. FITC and DOX fluorescence intensity detection of mitochondria isolated from HeLa cells after being treated with different MSNPs. (Reproduced with permission from reference ${ }^{210}$, Copyright 2015 Royal Society of Chemistry).

The obtained data in Figure 8 showed that in the control groups of MSNPs without fluorescence signal (MSNPs-NH 2 and MSNPs- $\mathrm{PPh}_{3}$ ), the fluorescence intensities were quite low because of no FITC functionality or DOX loading. In the groups of applying MSNPs without TPP conjugation (MSNPs- $\mathrm{NH}_{2}-\mathrm{DOX}$ and MSNPs- $\mathrm{NH}_{2}-\mathrm{FITC}$ ), the fluorescence signals of isolated mitochondria were still not significant even through slightly higher than non-fluorescent MSNPs groups. However, in the cases of applying TPP conjugated groups (MSNPs- $\mathrm{PPh}_{3}-\mathrm{DOX}$ and $\mathrm{MSNPs}-\mathrm{PPh}_{3}$-FITC), the fluorescence intensities of isolated mitochondria for both FITC and DOX were significantly higher than other testing groups, indicating that FITC and DOX molecules could be detected in isolated mitochondria in HeLa cells. These results directly demonstrated the existence of MSNPs-PPh ${ }_{3}-\mathrm{DOX}$ and MSNPs$\mathrm{PPh}_{3}$-FITC in isolated mitochondria form HeLa cells, also confirming the effective mitochondria targeting facilitated by TPP surface conjugation.

\subsubsection{Endocytosis of MSNPs}

It has been extensively documented that the cellular uptake process of MSNPs is lysosome directed endocytosis and MSNPs can be effectively internalized by mammalian cells 
including cancerous cells and normal cells through endocytosis. One of the features of effective drug delivery system is quick endocytosis process and effective escape from being trapped by lysosome. Therefore, in order to investigate the interactions between internalized MSNPs and cellular lysosome, confocal laser microscopy method was applied to visualize MSNPs loaded with DOX molecules and lysosomes. Here the cellular lysosomes were stained by Lyso-Tracker Green in green color. The DOX loaded and internalized MSNPs were shown as red color from the red fluorescence of DOX molecules.

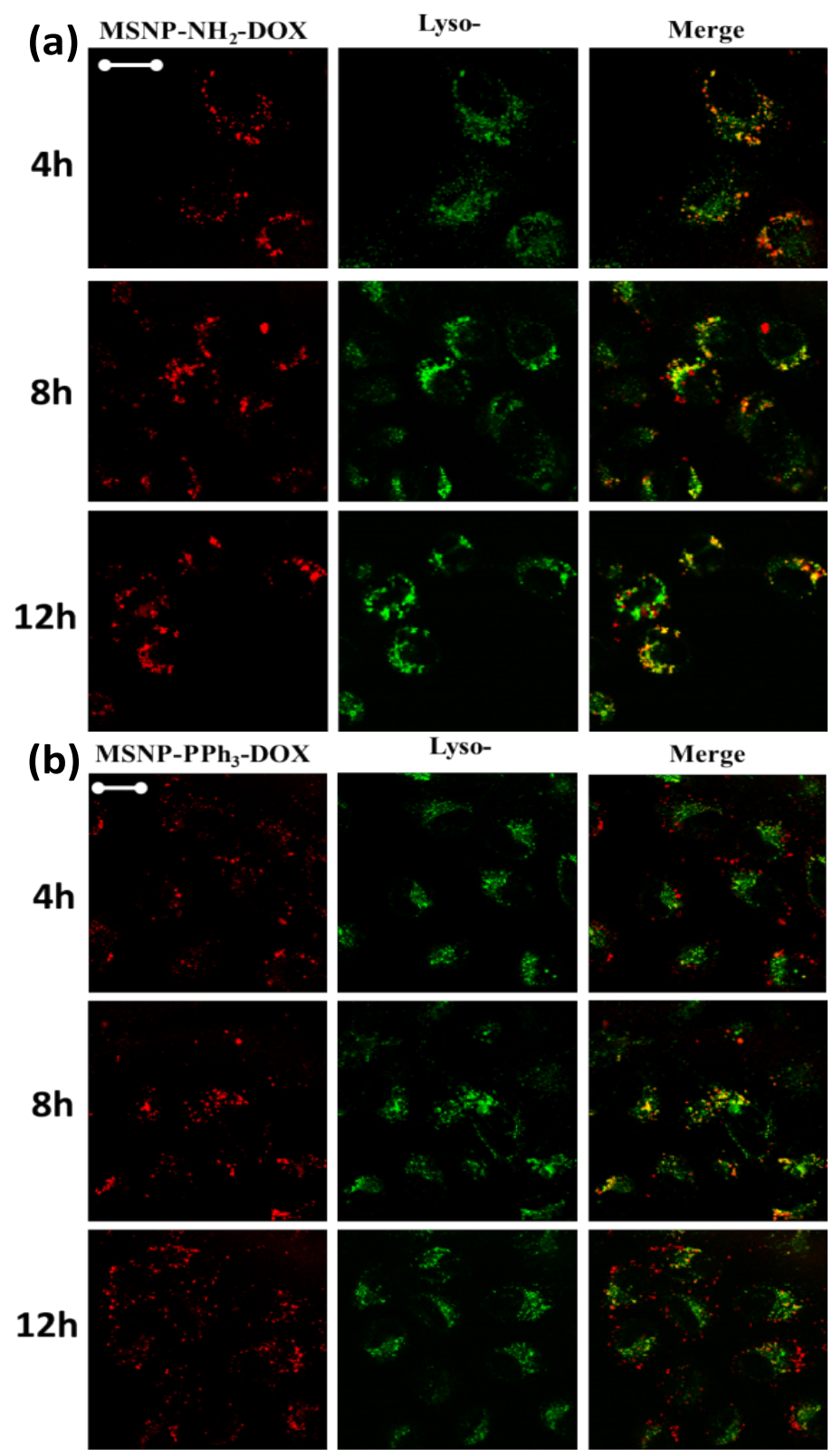

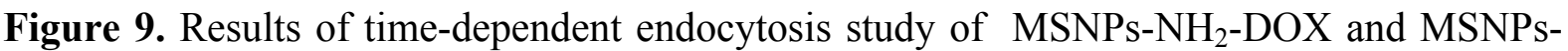
$\mathrm{PPh}_{3}$-DOX. Lysosomes were stained by Lyso-Tracker Green (50 nM). Scale bar: 
$20 \mu \mathrm{m}$.(Reproduced with permission from reference ${ }^{210}$, Copyright 2015 Royal Society of Chemistry).

From the obtained results (Figure 9), firstly, it was shown that red dots were well distributed inside cytoplasm of HeLa cells meaning that DOX loaded MSNPs were successfully internalized after incubating cells for just $4 \mathrm{~h}$ and the biocompatibility of MSNPs was good. Plus, the green colored dots could be also well visualized which means that cellular lysosomes of HeLa cells were successfully stained. After HeLa cells were treated with MSNPs- $\mathrm{NH}_{2}$-DOX for $4 \mathrm{~h}$, no obvious interactions between internalized MSNPs and lysosomes were observed as red colored spots and green colored spots were not co-localized in the merged image, indicating that MSNPs were not trapped yet by lysosomes after incubating cells with MSNPs-NH $\mathrm{N}_{2}$-DOX for $4 \mathrm{~h}$. After $8 \mathrm{~h}$ incubation of MSNPs- $\mathrm{NH}_{2}-\mathrm{DOX}$ with HeLa cells, clear yellow colored spots were observed in the merged imaged, stating that MSNPs- $\mathrm{NH}_{2}$-DOX were trapped already by cellular lysosomes at that time. Such trapping of MSNPs by lysosomes also proves the lysosome directed endocytosis process of MSNPs cellular internalization. After that, HeLa cells were further incubated with $\mathrm{MSNPs}-\mathrm{NH}_{2}-\mathrm{DOX}$ for of $12 \mathrm{~h}$. The confocal laser microscopy images, however, showed that the yellow colored spots could also be observed in the merged image, indicating that MSNPs- $\mathrm{NH}_{2}$-DOX were still trapped by lysosomes after $12 \mathrm{~h}$ incubation. Hence, MSNPs- $\mathrm{NH}_{2}-\mathrm{DOX}$ cannot effectively escape from lysosome trapping. Secondly, the interactions between $\mathrm{MSNPs}-\mathrm{PPh}_{3}$ DOX and cellular lysosomes in HeLa cells during endocytosis process were investigated as well. The results showed that similar to the case of applying MSNPs- $\mathrm{NH}_{2}-\mathrm{DOX}$, there were no obvious interactions between MSNPs- $\mathrm{PPh}_{3}$-DOX and lysosomes after $4 \mathrm{~h}$ incubation. Then, after further $4 \mathrm{~h}$ incubation ( $8 \mathrm{~h}$ in total), the yellow colored spots were found in the merged image which means that MSNPs- $\mathrm{PPh}_{3}-\mathrm{DOX}$ were trapped by lysosomes after $8 \mathrm{~h}$ incubation. Additionally, after another $4 \mathrm{~h}$ incubation (12 $\mathrm{h}$ in total), no obvious yellow colored spots could be observed in the merged image, indicating that $\mathrm{MSNPs}-\mathrm{PPh}_{3}-\mathrm{DOX}$ 
were not trapped by lysosomes at this time. Thus, MSNPs- $\mathrm{PPh}_{3}$-DOX were able to escape the trapping by lysosomes after $12 \mathrm{~h}$ incubation. Finally, in can be concluded that DOX loaded and TPP conjugated MSNPs exert to have effective escape from lysosome and quick endocytosis process in HeLa cells. The reasonable explanation of such observation lies in the TPP surface functionality of MSNPs. Specifically, TPP surface functionality can facilitate the transmembrane process of MSNPs owing to the high lipophilicity of TPP molecules. In addition, the quick endocytosis property of TPP conjugated MSNPs can also be helpful in the avoidance of pre-release of DOX molecules from $\mathrm{MSNPs}-\mathrm{PPh}_{3}$-DOX before reaching the target site, which further demonstrates the promising application potential of MSNPs in subcellular drug delivery.

\subsubsection{Cytotoxicity and mitochondrial malfunctions}

The pre-required property of effective drug delivery system is non-cytotoxicity. Basically, the effective anticancer property of DOX delivered by drug delivery system is achieved by the mechanism of enhanced intracellular DOX concentration, leading to the effective binding interaction between DOX molecules and DNA molecules in nucleus. However, in our designed MSNPs delivery system, DOX molecules are intended to be delivered into mitochondria in a targeted way, resulting in binding interaction between DOX molecules and mtDNA molecules other than DNA molecules in nucleus. All the designed effective drug delivery systems should present non-cytotoxicity. Therefore, the cytotoxicity of pure MSNPs and DOX loaded MSNPs were investigated by applying MTT (3-(4,5-dimethylthiazol-2-yl)2,5-diphenyltetrazolium bromide) assay. MTT is chemical compound that can selectively bind with corresponded compound produced by active cells and the resulted compound can be dissolved in DMSO to give purple color. Darker purple color means the higher cellular activity. The final cytotoxicity is represented by survival ratio. 

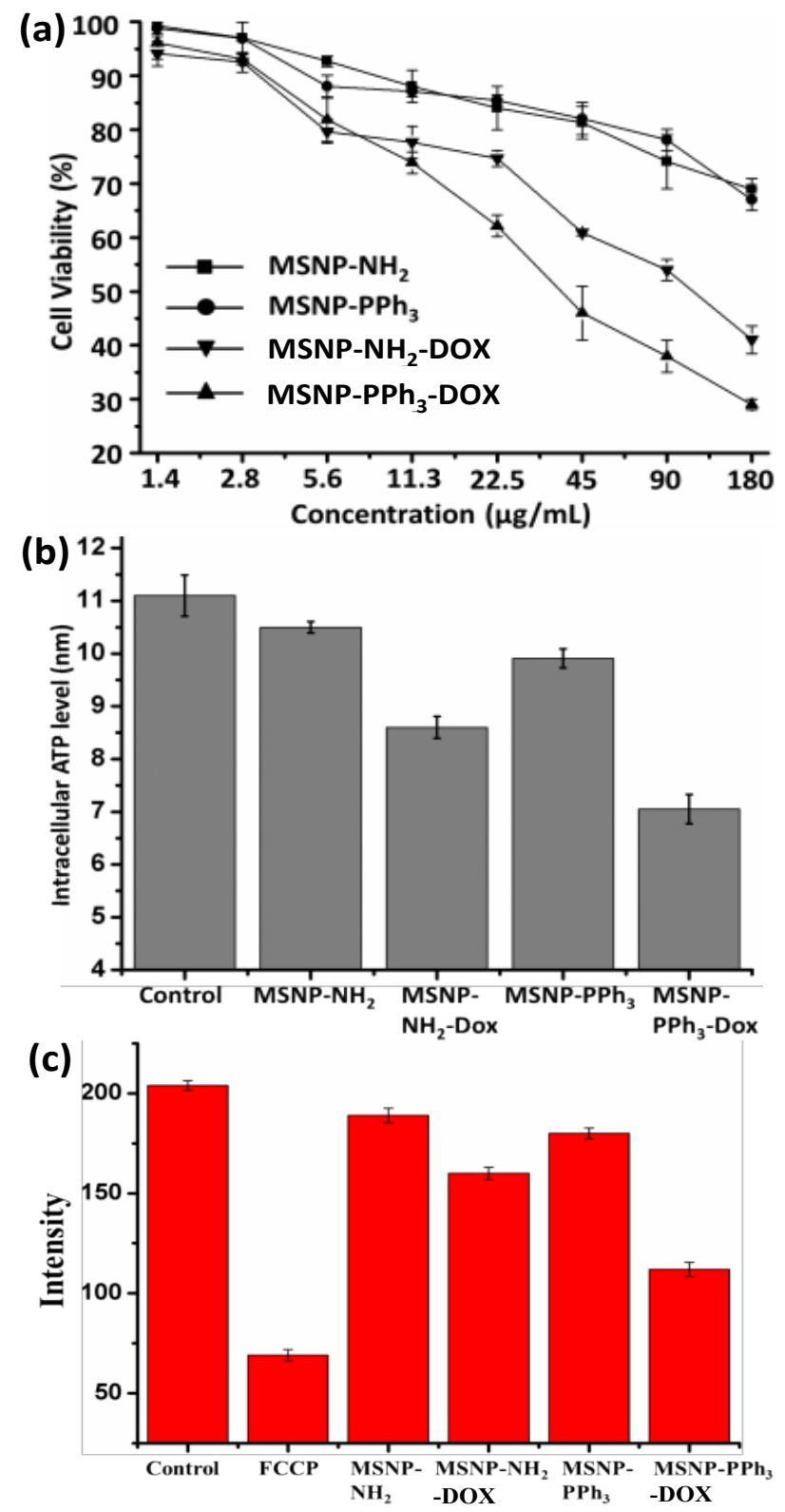

Figure 10.Cell viability (a) and intracellular ATP level in HeLa cells (b) after being treated with different MSNPs $(50 \mu \mathrm{g} / \mathrm{mL})$ for $24 \mathrm{~h}$. (c) Mitochondria membrane potential detection after treating HeLa cells with different MSNPs $(50 \mu \mathrm{g} / \mathrm{mL}$ ) and FCCP (positive control) for 24 h. (Reproduced with permission from reference ${ }^{210}$, Copyright 2015 Royal Society of Chemistry).

In the obtained results of cytotoxicity study (Figure 10a), more than $70 \%$ of HeLa cells were alive when treating them with pure $\mathrm{MSNPs}-\mathrm{NH}_{2}$ and $\mathrm{MSNPs}-\mathrm{PPh}_{3}$ even at high concentration of $180 \mu \mathrm{g} / \mathrm{mL}$ after $24 \mathrm{~h}$ incubation, indicating that no obvious cytotoxicity was observed for both MSNPs-PPh 3 and MSNPs-NH 2 . For DOX loaded MSNPs, both MSNPs$\mathrm{NH}_{2}$-DOX and MSNPs-PPh ${ }_{3}$-DOX showed effective cytotoxicity towards HeLa cells in the 
concentration range from 11.3-180 $\mu \mathrm{g} / \mathrm{mL}$. However, it was observed that $\mathrm{MSNPs}-\mathrm{PPh}_{3}-\mathrm{DOX}$ exhibited better cytotoxicity effect than that of MSNPs- $\mathrm{NH}_{2}-\mathrm{DOX}$ in that the IC 50 values for MSNPs-NH ${ }_{2}$-DOX and MSNPs-PPh ${ }_{3}$-DOX in HeLa cells were $122.6 \mu \mathrm{g} / \mathrm{mL}$ and $31.5 \mu \mathrm{g} / \mathrm{mL}$, respectively. Such effect demonstrates that TPP conjugated MSNPs with DOX loaded have better anticancer effect than MSNPs without conjugation of mitochondrial targeting ligand. The possible explanation for the observed results should be closely related with TPP functionality. The resulted function of TPP surface conjugation is effectively delivery DOX molecules to mitochondria by MSNPs, leading to the binding of mtDNA and DOX molecules and further dysfunctions of mitochondria. In contrast, the anti-cell proliferating effect of MSNPs- $\mathrm{NH}_{2}$-DOX is mainly due to the binding interaction of DOX molecules and nucleus DNA molecules. It is fully documented that the functions of mtDNA are closely realted to the normal functions of mitochondria such as ATP production and mitochondrial membrane potential. What's more, binding of mtDNA and DOX molecules process should be more effective than the binding of nucleus DNA and DOX molecules owing to the effective escape from lysosome trap and quick endocytosis process of MSNPs- $\mathrm{PPh}_{3}-\mathrm{DOX}$. Therefore, the

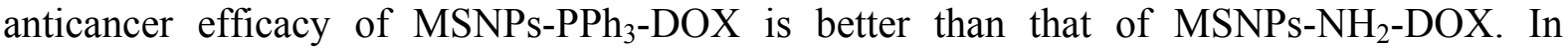
addition, the results of cytotoxicity study also indicate the sensitivity and importance of mitochondria inside cancerous cells.

Furthermore, the influences of DOX loaded MSNPs towards intracellular ATP production level as well as mitochondrial membrane potential were investigated due to the close relation between mtDNA and effective ATP production as well as the maintenance of high mitochondrial membrane potential. Here we applied flow cytometry method to quantitatively characterize the malfunctions caused by DOX loaded MSNPs. The intracellular ATP production level was calculated based on the standard curve obtained by detecting intensities from a series of different ATP concentrations. The mitochondrial membrane potential 
staining process was conducted according to the staining kit provided by Invitrogen. Tetramethylrhodamine ethyl ester (TMRE) chemical compound was applied to stain the mitochondria with normal active membrane potential. Higher intensity of TMRE indicates more active of mitochondria. Carbonyl cyanide 4-(trifluoromethoxy) phenyl-hydrazone (FCCP) chemical compound was used as positive control due to its inhibition effect against mitochondrial oxidative phosphorylation. Once cells are treated with FCCP, the active mitochondrial membrane potential will be affected and lost, leading to the unsuccessful staining of mitochondria by TMRE.
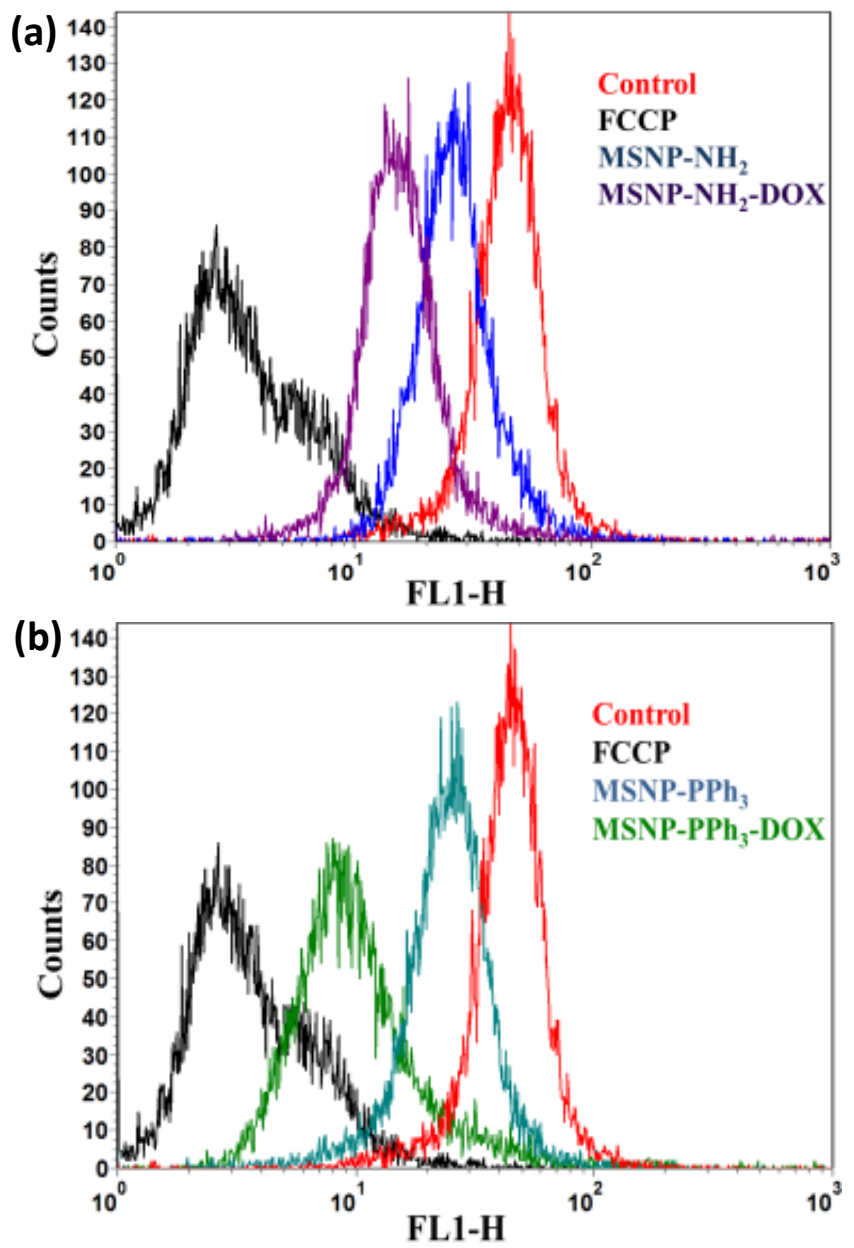

Figure 11. Flow cytometry analysis of HeLa cells after being treated with different MSNPs $(50 \mu \mathrm{g} / \mathrm{mL})$ and FCCP (positive control) for $24 \mathrm{~h}$. FL1-H: fluorescence intensity of TMRE. (Reproduced with permission from reference ${ }^{210}$, Copyright 2015 Royal Society of Chemistry). 
As seen in the obtained results, in the study of intracellular ATP production level detection (Figure 10b), HeLa cells treated with MSNPs-NH $\mathrm{NH}_{2}$ and MSNPs-PPh 3 did not show the obvious down regulated level of intracellular ATP production, meaning that pure MSNPs would not affect intracellular ATP production. In the case of treating cells with DOX loaded MSNPs, both MSNPs-NH $\mathrm{N}_{2}$-DOX and MSNPs-PPh${ }_{3}$-DOX presented lowered level of intracellular ATP production. However, much more lowered ATP production level was observed in MSNPs- $\mathrm{PPh}_{3}$-DOX group than that in MSNPs- $\mathrm{NH}_{2}$-DOX groups, indicating that TPP conjugated MSNPs would be more effective in inhibiting intracellular ATP production. That is to say, MSNPs- $\mathrm{PPh}_{3}$-DOX will lead to effective mitochondrial malfunctions. Similarly, in the study of mitochondrial membrane potential detection (Figure 10c), pure MSNPs without DOX loading did not present obvious effect in decreasing mitochondrial membrane potential. Whereas in the testing of DOX loaded MSNPs, $\mathrm{MSNPs}-\mathrm{PPh}_{3}-\mathrm{DOX}$ showed more effective function in decreasing mitochondrial membrane potential which means that DOX molecules that delivered into mitochondria could lead to direct depletion of mitochondrial membrane potential. Similar results were also obtained by applying flow cytometry study (Figure 11). The FCCP positive groups showed the most effective decrease of TMRE fluorescence intensity. For experimental group of using MSNPs without TPP conjugation, DOX loaded MSNPs did not show obvious effect in decreasing TMRE fluorescence intensity, meaning that DOX molecules would not affect mitochondrial membrane potential when delivered to cytoplasm. However, in the case of applying TPP conjugated MSNPs, mitochondrial membrane potential would be greatly affected and decreased, indicating that DOX molecules delivered into mitochondrial were effective in depleting mitochondrial membrane potential. Above all, in can be concluded that DOX molecules delivered by MSNPs to mitochondria can effectively induce cytotoxicity and mitochondrial malfunctions. 


\subsection{Conclusion}

In summary, well-ordered MSNPs with proper size for biological application were successfully fabricated. New surface functionalization of TPP mitochondrial targeting groups were further successfully conjugated on MSNPs through simply one-step reaction to give $\mathrm{MSNPs}-\mathrm{PPh}_{3}$. The effectiveness of $\mathrm{MSNPs}-\mathrm{PPh}_{3}$ in mitochondrial targeting was characterized by confocal laser microscopy study, showing that MSNPs$\mathrm{PPh}_{3}$ were effective in co-localizing with mitochondria in HeLa cancerous cells. The synthesized MSNPs were capable of loading DOX drug molecules with high loading capacity. In addition, DOX molecules could be effectively delivered into mitochondria by TPP conjugated MSNPs, which was demonstrated by mitochondria isolation study. MSNPs- $\mathrm{PPh}_{3}$ were observed to be able to effectively escape from lysosome trap during endocytosis process, making them more effective as drug delivery system. The cytotoxicity study showed that pure MSNPs presented good biocompatibility and DOX delivered into mitochondria by MSNPs exhibited better anticancer effect than DOX delivered to cytoplasm. More importantly, mitochondrial malfunctions such as decreased ATP production level and lose of mitochondrial membrane potential caused by MSNPs-PPh ${ }_{3}$-DOX were observed. As a result, the presented current research indicates the application potential of MSNPs in sub-cellular drug delivery. In addition, our mitochondrial targeted MSNPs system can be further applied in delivering mitochondrial specific drug molecules such as Rotenone as well as other hydrophobic drug molecules in order to achieve the maximized anticancer efficiency. 


\section{Chapter 3: Anticancer Effect of $\alpha$-Tocopheryl Succinate Delivered by Mitochondria-Targeted Mesoporous Silica Nanoparticles}

\subsection{Introduction}

Up to date, the understanding of cancer has been deeper than ever before. It has been accepted that, generally, cancer cells share six common hallmarks that make them very unique and different from normal cells. Such common shared hallmarks of cancer cells involve: (1) Cancer cells are able to stimulate cells growth by themselves (Self-sufficiency in growth signal). (2) Cancer cells tend to be not sensitive to inhibitory signals that may direct them to stop cell dividing (Insensitivity to anti-cell growth signals). (3) Cancer cells can escape from the programmed cell death (Evading apoptosis). (4) They have the ability to duplicate themselves infinitely (Unlimited cell proliferation). (5) They are capable of stimulating the generation and growth of blood vessels to obtain nutrient supply (Sustained angiogenesis). (6) They can invade local tissues and transfer to other distant site to growth (Tissue invasion and metastasis). Among such mentioned features of cancer cells, the unlimited cell proliferation property is the foundation of other hallmarks. Basically, The proliferation of cells highly depends on the energy supply by adenosine triphosphate (ATP) that is mostly generated in mitochondria, the critical organelle in both normal cells and cancerous cells. However, mitochondria in cancerous cells act quite differently from that in normal cells. One of the major differences is that the efficiency of producing ATP molecule in cancerous cells is lower than that in normal cells. The reason behind is that the energy production in cancerous cells depends on glycolysis process, which is called the "Warburg effect", one of the indicators of cancer. Paradoxically, cancerous cells consume more energy to maintain a high rate of cell dividing, which means that cancerous cells require more mitochondria than normal cells. In addition, mitochondria in cancerous cells are more 
sensitive to mitocans, the drugs with active targeting to mitochondria. Therefore, mitochondria as targeting sites have drawn more attention in cancer therapy.

In light of the importance of mitochondria, mitocans, termed as drug molecules with an active site to mitochondria, have been developed. As discussed before, $\alpha$-tocopheryl succinate $(\alpha-$ TOS $)$ is one of the most effective mitocans in inducing the apoptosis. As one form of vitamin $\mathrm{E}$ analogues, $\alpha$-TOS is an inhibitive competitor of ubiquinone, displacing and preventing ubiquinone from binding to succinate dehydrogenase (SDH) in order to result in the disorder in the electron transportation chain and further induce the mitochondria destabilization and intrinsic apoptosis. More importantly, it was found that $\alpha$-TOS could selectively kill cancerous cells with little or no side effects to normal cells. However, the main drawback preventing $\alpha$-TOS from its wide application in clinical treatment is its hydrophobic property. In most studies regarding the anticancer effect of $\alpha$-TOS in vitro and in vivo, organic solvents like dimethyl sulfoxide (DMSO) and ethanol are applied as the solvent, making it hard to avoid side effects. Therefore, it is essential to develop $\alpha$-TOS delivery systems to overcome the side effects. Up to date, there are only limited discussions regarding the delivery of $\alpha$-TOS based on nanocarriers. In this work, applying mesoporous silica nanoparticles (MSNPs) in the delivery of $\alpha$-TOS to mitochondria in cancerous cells was investigated for the first time.

MSNPs have been extensively investigated concerning the application of drug delivery as mentioned before. With the merits of high surface area, high pore volume, and tunable pore and particle size, MSNPs could be impressively loaded with drug molecules having different molecule sizes. In addition, MSNPs were reported to be biocompatible. Compared with other delivery systems such as polymers, MSNPs are easy to fabricate and commercially low cost. Moreover, MSNPs are also known as effective delivery carriers for hydrophobic drug molecules, making it more suitable for delivering $\alpha$-TOS. 211, 212 209, 210 211, 212 
Various mitochondria targeting MSNPs were developed. The mitochondria targeting MSNPs were characterized and further loaded with $\alpha$-TOS drug molecules. After that, the anticancer efficiency of fabricated MSNPs was conducted against HeLa and HepG2 cancer cell lines as well as HEK293 normal cell line. The aim of the current work is to improve biological activity of $\alpha$-TOS by applying simple mitochondria targeted targeting ligands have been applied, including chemical compounds such as triphenoylphosphonium (TPP) and peptides like XJB peptides and SS peptides. Having three phenyl groups, TPP is normally positively charged, thus attracting highly negatively charged mitochondria. In addition, TPP with sufficient lipophilicity can facilitate the process of trans-membrane. Furthermore, the attractive performance of TPP in targeting mitochondria has been fully developed. In this work, TPP was employed in the surface functionalization to obtain MSNPs based delivery system with high efficiency. The mitochondria targeting MSNPs were characterized and further loaded with $\alpha$-TOS drug. After that, the anticancer efficiency of fabricated MSNPs was conducted against HeLa and HepG2 cancer cell lines as well as HEK293 normal cell line. The aim of the current work is to improve biological activity of $\alpha$-TOS by applying simple mitochondria targeted MSNP delivery system with high efficiency (Figure 1).

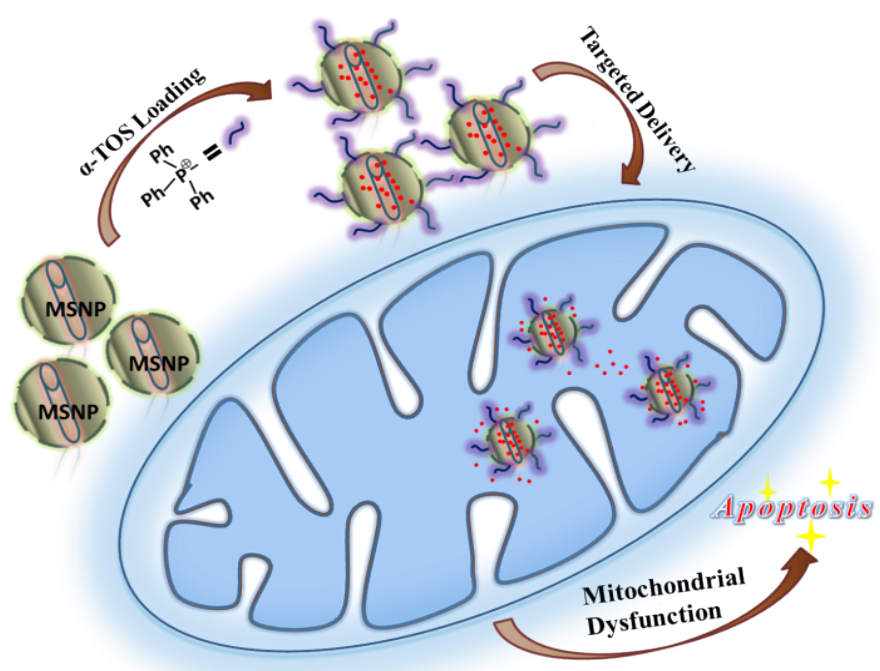


Figure 1.Schematic diagram of $\alpha$-TOS delivery by mitochondria targeted MSNPs.

\subsection{Experimental materials and methods}

\subsubsection{Applied materials and instruments}

Materials and reagents: Absolute ethanol (EtOH, >99.9\%), 3aminopropyltriethoxysilane (APTS), (4-carboxybutyl) triphenylphosphonium bromide (98\%), Caspase 3 Detection Kit (Colorimetric, Abcam), Caspase 9 Detection Kit (Colorimetric, Abcam), cetyltrimethylammonoium bromide (CTAB, 90\%), 4',6diamidino-2-phenylindole (DAPI), Mitochondrial oxidative phosphorylation inhibitor 99\% (FCCP, Abcam), (3-(4,5-dimethylthiazol-2-yl)-2,5-diphenyltetrazolium bromide (MTT), dulbecco's modified eagle's medium (DMEM), 1-ethyl-3-(3dimethylaminopropyl) carbodiimide (EDC), fetal bovine serum (FBS), fluorescein isothiocyanate (FITC), hydrochloride ( $\mathrm{HCl}, 37 \%$ ), N-hydroxysulfosuccinimide (NHS), intracellular ATP determination kit (Invitrogen), Lyso-Tracker Red (Invitrogen), methanol $(\mathrm{MeOH}, 99.5 \%)$, mitochondria isolation kit for cultured cells (Thermo Scientific), Mito-Tracker Deep Red (Invitrogen), phosphate buffered saline (PBS), sodium hydroxide $(\mathrm{NaOH})$, tetraethylorthosilicate (TEOS, 99\%), and $\alpha$-tocopheryl succinate $(\alpha$-TOS) were purchased commercially.Nanopure water (18.2 M $\Omega$, Millipore Co., USA) was used in all experiments and buffer preparations.

Instruments: Transmission electron microscopy (TEM) images were collected by using a JEOL JEL-1400 at $100 \mathrm{kV}$. Field emission electron microscopy (FE-SEM) images were obtained by using a FE-SEM 6340 (JEOL) at $5 \mathrm{kV}$. Specific surface areas and pore size distributions of the as-fabricated MSNPs were measured by using ASAP-2020 Mircomeritics. Powder X-ray diffraction patterns were collected by using aX'Pert Powder X-ray diffractometer. Zeta potentials were measured by using a MavernNanosizer. FT-IR spectra 
were measured by using a Fourier Transformed Infrared Spectrometer. UV/Vis absorption spectra were recorded by using UV/Vis 2501 spectrometer as well as NanoDrop spectrophotometer (2000/2000c, Thermo Scientific). A micro-plate reader (infinite 200 PRO, Tecan) was employed for the MTT assay, luminescence and fluorescence analysis. Confocal laser scanning microscopy images were taken by using a confocal microscope (Leica TCS SP5, 63× oil objective).

\subsubsection{Fabrication of MSNPs}

MSNPs with amine surface functionalization were synthesized following the conventional sol-gel method. Briefly, $\mathrm{NaOH}$ aqueous solution $(2.0 \mathrm{M})$ was first prepared. In the meantime, CTAB $(500 \mathrm{mg})$ was dissolved in deionized water $(240 \mathrm{~mL})$. After that, the prepared $\mathrm{NaOH}$ aqueous solution $(1.1 \mathrm{~mL})$ was added into the CTAB solution. Then, the mixture solution was heated to $80{ }^{\circ} \mathrm{C}$ under vigorous stirring until the temperature of the mixture solution was stabilized at $80{ }^{\circ} \mathrm{C}$. Under vigorous stirring, TEOS $(2.0 \mathrm{~mL})$ was added dropwise into the reaction solution. After $15 \mathrm{~min}$, APTES $(0.5 \mathrm{~mL})$ was added dropwise into the mixture solution. The mixture solution was stirred at $80^{\circ} \mathrm{C}$ for another $2 \mathrm{~h}$. The formed nanoparticles were collected by centrifuging the reaction solution at $8000 \mathrm{rpm}$ for $10 \mathrm{~min}$, followed by washing with deionized water and methanol for three times. Finally, the obtained MSNPs were dried under vacuum.

The removal of CTAB was conducted the same as described in Chapter 2.

The conjugation of TPP on the surface of MSNPs was carried out before the CTAB removal in order to give selective functionalization on the outer surface. Generally, TPP (50 mg) was dissolved in deionized water $(5 \mathrm{~mL})$. Then, 1-ethyl-3-(3-dimethylaminopropyl) carbodiimide (EDC, $25 \mathrm{mg}$ ) and N-hydroxysulfosuccinimide (NHS, $35 \mathrm{mg}$ ) were added to form the mixture solution. The activation of the carboxylic acid group on TPP was carried out by 
stirring the mixture solution in the dark for $2 \mathrm{~h}$. At the same time, CTAB-containing MSNPs$\mathrm{NH}_{2}(10 \mathrm{mg})$ was suspended in deionized water $(10 \mathrm{~mL})$. Then, the solution with activated TPP was added into the MSNPs suspension. Obtained solution was stirred for $48 \mathrm{~h}$. The obtained MSNPs-PPh 3 was collected by centrifugation at $8000 \mathrm{rpm}$ for $10 \mathrm{~min}$, and washed with methanol and deionized water thoroughly for three times. The removal of CTAB from the obtained MSNPs-PPh ${ }_{3}$ was conducted using a method similar to the case of $\mathrm{MSNPs}-\mathrm{NH}_{2}$ described above.

FITC labeled MSNPs were also prepared. The detailed process can be referred in Chapter 2.

\subsection{3 $\alpha$-TOS loading}

At first, the $\alpha$-TOS stock solution was prepared at a concentration of $2 \mathrm{mg} / \mathrm{mL}$. Then, MSNPs-NH $\mathrm{NH}_{2}(2.0 \mathrm{mg})$ and $\mathrm{MSNPs}-\mathrm{PPh}_{3}(2.0 \mathrm{mg})$ were suspended in the $\alpha$-TOS stock solution $(2 \mathrm{mg} / \mathrm{mL})$ respectively, and the mixture solution was stirred in the dark for $24 \mathrm{~h}$. After that, $\alpha$-TOS loaded MSNPs were collected by centrifugation at $10000 \mathrm{rpm}$ for $5 \mathrm{~min}$. In order to eliminate free $\alpha$-TOS molecules, $\alpha$-TOS loaded MSNPs were re-collected after resuspending them in methanol. The loading capacity was calculated based on the difference of UV-vis absorption intensity at $284 \mathrm{~nm}$ between the original $\alpha$-TOS stock solution and the supernatant after the drug loading. The drug loading experiment was repeated for three times independently for statistical analysis.

\subsubsection{Cell culture}

HeLa (cervical cancer cell line), HepG2 (human liver cancer cell line) and HEK293 (Human Embryonic Kidney 293 cell line) were cultured in Dulbecco's Modified Eagle Medium (DMEM) containing $10 \%$ fetal bovine serum (Invitrogen) and 1\% penicillin/streptomycin (Invitrogen) under the humidified condition containing $5 \% \mathrm{CO}_{2}$ at $37^{\circ} \mathrm{C}$. 


\subsubsection{MTT cytotoxicity study}

In general, HeLa cells, HepG2 cells and HEK 293 normal cells were cultured in good condition with good cellular status. The detailed detection process of each cell line was conducted the same as in Chapter 2.

\subsubsection{Confocal laser microscopy study}

In general, HeLa cells, HepG2 cells and HEK 293 normal cells were cultured in good condition with good cellular status. One 6-well plate was filled with cover sides in each well at first. Different cells were then seeded in the 6 -well plant at a cell density of $1 \times 10^{5}$ per well and $2.0 \mathrm{~mL}$ of culturing medium was added in each well. The detailed detection process can be referred in Chapter 2 .

The fluorescence of Lyso-Tracker Green and DAPI was excited at $\lambda=488 \mathrm{~nm}$ and $\lambda=405$ $\mathrm{nm}$, respectively. The fluorescence of Mito-Tracker Deep Green and Red was excited at $\lambda=$ $488 \mathrm{~nm}$ and $\lambda=594 \mathrm{~nm}$, respectively. The fluorescence intensity of FITC was collected by being excited by laser with wavelength of $488 \mathrm{~nm}$. The fluorescence of DAPI, FITC, MitoTracker Deep Red, Mito-Tracker Green, and Lyso-Tracker Green was collected at $\lambda=$ $(460 \pm 10), \quad(520 \pm 10), \quad(620 \pm 10), \quad(520 \pm 10), \quad$ and $(530 \pm 10)$ channels, respectively. All fluorescence channels were detected sequentially.

\subsubsection{Flow cytometry study}

The cellular uptake of different MSNPs (with FITC functionality) was studied in HeLa and HepG2 cells by using flow cytometry. Briefly, HeLa and HepG2 cells were seeded in 6-well plate at the cell density of $1 \times 10^{5}$ per well and further incubated for $24 \mathrm{~h}$. Then, old medium was replaced by a fresh medium containing MSNPs-FITC, and cells were further incubated for another $24 \mathrm{~h}$. After that, old medium was removed and cells were washed with a fresh 
medium for three times. Cells were harvested in $2.0 \mathrm{~mL}$ tube with the treatment of trypsin and collected by centrifugation. In order to eliminate free MSNPs, cells were re-suspended in PBS buffer and re-collected by centrifugation in a new $2.0 \mathrm{~mL}$ tube. Finally, these cells were ready for flow cytometry study.

\subsubsection{Mitochondria isolation}

Mitochondria isolation was conducted in HeLa, HepG2 and HEK293 cells. The isolation process was carried out based on the mitochondria isolation kit for cultured cells (Thermo Scientific). Briefly, different cells were cultured in T-75 cell culture flask at a density of $1 \times 10^{7}$. After culturing in a good condition, cells were harvested by the trypsin treatment. After that, cells were pelleted by centrifuging cell suspension in a $2.0 \mathrm{~mL}$ micro-centrifuge tube at $850 \times \mathrm{g}$ for $2 \mathrm{~min}$. Pelleted cells were treated with cell rupturing reagent $(800 \mu \mathrm{L})$ provided in the isolation kit. Then, the tube with cells was vortexed for 10 seconds in a medium speed, and placed with ice for $2 \mathrm{~min}$. The tube was added with mitochondria isolation reagent $(10 \mu \mathrm{L})$, and further treated under vortex for 10 seconds. After incubating the tube on ice for $5 \mathrm{~min}$, the isolation reagent $(800 \mu \mathrm{L})$ was added into the cell-containing tube, and the tube was gently shaken for several times. The tube was centrifuged at $700 \times \mathrm{g}$ at $4{ }^{\circ} \mathrm{C}$ for $10 \mathrm{~min}$, and then the supernatant was transferred to a new tube $(2.0 \mathrm{~mL})$. The mitochondria-containing pellet was suspended in PBS $(200 \mu \mathrm{L})$ to form the PBS buffer with mitochondria. The fluorescence intensity at an excitation wavelength of $488 \mathrm{~nm}$ was measured by using micro-plate reader (infinite 200 PRO, Tecan). The UV absorption intensity of $\alpha$-TOS was measured by using NanoDrop spectrophotometer (2000/2000c, Thermo Scientific). 


\subsubsection{Intracellular ATP production level detection}

Intracellular ATP level detection in HeLa and HepG2 cells was conducted by using ATP determination kit (Invitrogen), and the detection process was carried out following the protocol provided. Generally, HeLa and HepG2 cells were seeded in a 6-well plate at a cell density of $1 \times 10^{5}$ per well and further cultured in a good condition for $24 \mathrm{~h}$. After that, old medium was replaced by a fresh medium $(2.0 \mathrm{~mL}$ per well) containing different MSNP samples. After the incubation for another $24 \mathrm{~h}$, cells were washed with PBS for two times and then treated with cell lysis buffer $(200 \mu \mathrm{L}$, Invitrogen). Cell debris was collected as ATPcontaining experimental samples, and the luminescence intensities of different samples after the treatment with the reaction substrate provided in the detection kit were observed. The quantification of intracellular ATP level was achieved by the calculation based on the standard curve obtained by measuring serious concentrations of ATP solutions. Rotenone was set as a positive control.

\subsubsection{Mitochondria membrane potential detection}

The mitochondria membrane potential of HeLa and HepG2 cancerous cells were detected by applying TMRE Mitochondrial Membrane Potential Assay Kit (Abcam). The detection process was carried out following procedures recommended by Abcam. Briefly, HeLa and HepG2 cells were seeded in a 6 -well plate at a cell density of $1 \times 10^{5}$ per well. Then, the plate was incubated for $24 \mathrm{~h}$. After that, the old medium was replaced by a fresh medium containing MSNP testing samples. After the incubation for another $24 \mathrm{~h}$, the medium was replaced again with a fresh medium containing TMRE (final concentration of $200 \mathrm{~nm}$ ). Cells were incubated at $37{ }^{\circ} \mathrm{C}$ for further $20 \mathrm{~min}$. Then, the fluorescence of TMRE was measured at an excitation wavelength of $549 \mathrm{~nm}$ by using micro-plate reader (infinite $200 \mathrm{PRO}$, Tecan). 
In addition, the mitochondria membrane potential was also detected by using flow cytometry method. Generally, HeLa and HepG2 cells were seeded in a 6-well plate at a cell density of $1 \times 10^{5}$ per well and further incubated at a good condition for $24 \mathrm{~h}$. Then, old medium was replaced by a fresh medium containing MSNPs samples. After further incubation of $24 \mathrm{~h}$, the old medium was removed and a fresh medium with TMRE (final concentration of $200 \mathrm{~nm}$ ) was added. Similarly, cells were further incubated for $20 \mathrm{~min}$. Then, cells were harvested by the treatment of trypsin and collected by centrifugation. After washing with PBS for two times and harvesting again in $2.0 \mathrm{~mL}$ tube, cells were ready for flow cytometry study. The flow cytometry data were obtained by using BD FACS Calibur Flow Cytometer. FCCP was set as a positive control.

\subsubsection{Caspase study}

The activities of caspase 9 and caspase 3 in HeLa and HepG2 cells were detected by using Caspase 9 Assay Kit (Colorimetric, ab65608) and Caspase 3 Assay Kit (Colorimetric, ab39401) provided by Abcam, respectively. The detection procedures were carried out following the protocol from Abcam. Briefly, HeLa and HepG2 cells were seeded in 6-well plate at a cell density of $1 \times 10^{5}$ per well and further cultured for $24 \mathrm{~h}$. After that, old medium was replaced with a fresh medium containing MSNP testing samples. Cells were harvested by the treatment of trypsin and pelleted in $2.0 \mathrm{~mL}$ tube. Then, pelleted cells were re-suspended in $50 \mu \mathrm{L}$ cold cell lysis buffer and incubated on ice for $10 \mathrm{~min}$. The cells lysis solution was centrifuged at $10,000 \times \mathrm{g}$ for $1 \mathrm{~min}$, and the supernatant was transferred to a fresh tube for the assay detection.

The caspase reaction buffer was prepared according to the provided protocol. The supernatant was added into 96 -well plate by $50 \mu \mathrm{L}$ per well. Then, reaction buffer $(50 \mu \mathrm{L})$ along with the substrate $(5 \mu \mathrm{L}, 4 \mathrm{mM})$ was added into the tested well. The well was gently 
mixed, and incubated at $37{ }^{\circ} \mathrm{C}$ for $1.5 \mathrm{~h}$. Finally, the O.D. intensity (405 nm) of each tested well was measured by micro-plate reader (infinite 200 PRO, Tecan).

\subsection{Results and discussion}

\subsubsection{Fabrication and characterization of MSNPs}

Amine group functionalized MSNPs $\left(\mathrm{MSNPs}-\mathrm{NH}_{2}\right)$ and TPP functionalized MSNPs $\left(\mathrm{MSNPs}-\mathrm{PPh}_{3}\right)$ were fabricated by following surfactant-directed method. The surfactant applied here is cetrimonium bromide (CTAB). The surface functionalization of amine group on MSNPs was obtained by adding 3-aminopropyl triethoxysilane (APTES) during the formation of mesoporous channels. The TPP groups were functionalized on the surface of MSNPs- $\mathrm{NH}_{2}$ through the reaction between carboxylic acid groups of TPP and amino groups of MSNPs-NH . With the aim to track MSNPs in vitro, fluorescein isothiocyanate (FITC) conjugated MSNPs (MSNPs-NH $\mathrm{N}_{2}$-FITC and MSNPs-PPh ${ }_{3}$-FITC) were fabricated based on the co-condensation method, which showed light yellow color.

(a)

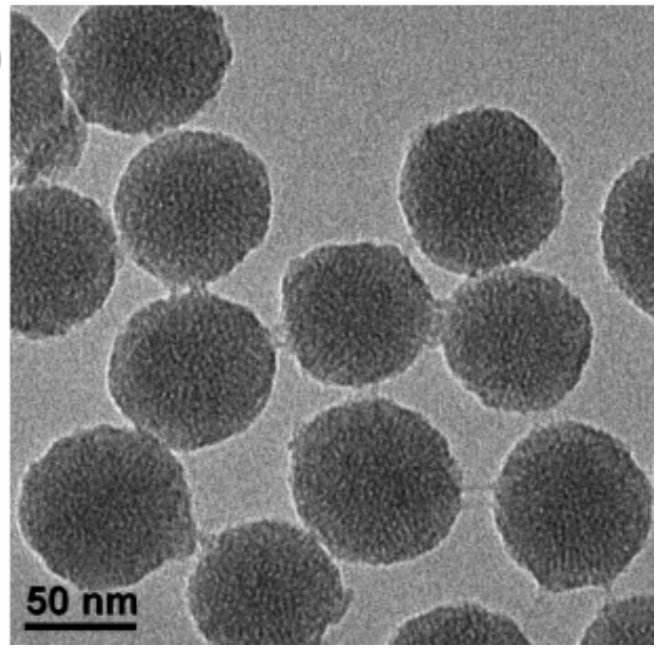

(b)

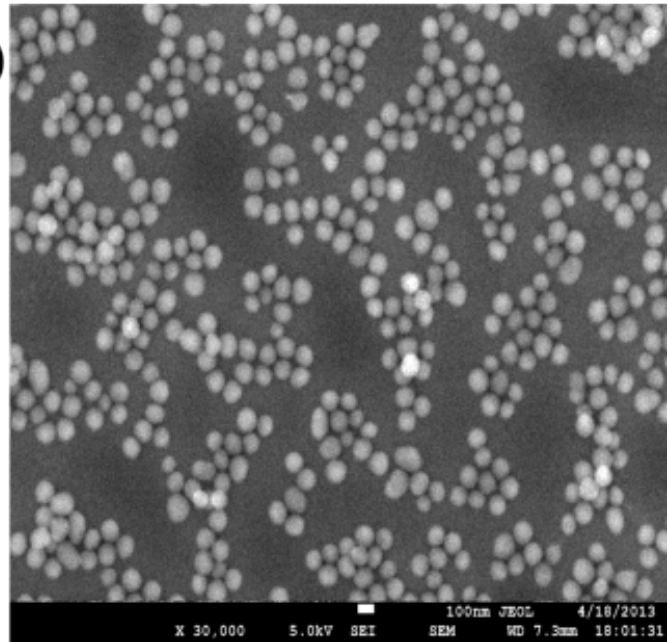

Figure 2.(a) TEM and (b) FE-SEM images of prepared MSNPs- $\mathrm{PPh}_{3}$.

Figure 2 shows transmission electron microscopy (TEM) and field emission scanning electron microscopy (FE-SEM) images of synthesized MSNPs-PPh $\mathrm{P}_{3}$. Regular spherical structured nanoparticles were observed. The average diameter of obtained nanoparticles was 
around $65 \mathrm{~nm}$, which was measured based on the calculation of fifteen nanoparticles from TEM images. Dynamic light scattering (DLS) method was also applied in characterizing the size of nanoparticles. Larger size of $115 \mathrm{~nm}$ was indicated from DLS experiments possibly because of inevitable swelling of some nanoparticles (Figure 3). It should be noted that comparing with MSNPs applied in Chapter 1, the nanoparticle average diameter is reduced from $80 \mathrm{~nm}$ to $65 \mathrm{~nm}$. This result is due to the reduced amount of sodium hydroxide solution added into the reaction mixture.

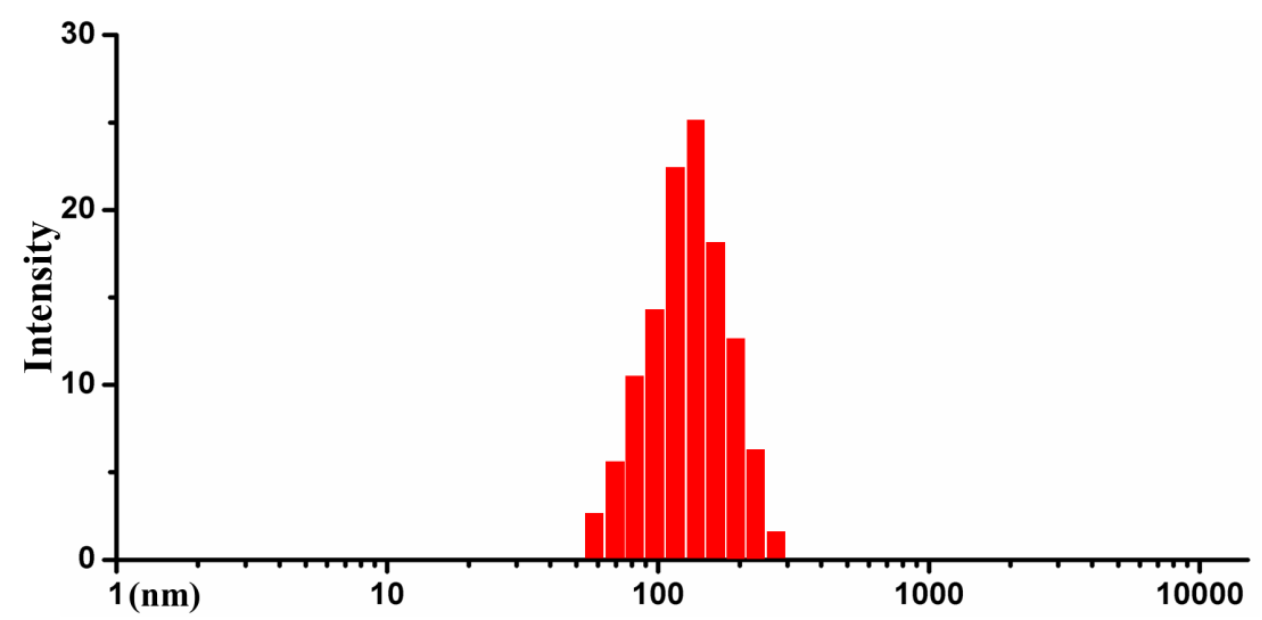

Figure 3.DLS analysis of MSNPs- $\mathrm{PPh}_{3}$ in $\mathrm{PBS}$ solution.

Fourier transform infrared (FT-IR) spectroscopy and Zeta potential measurements were used to characterize the successful surface functionalization of amine and TPP groups. As shown in Figure 4b, similar to the data obtained in chapter 1 , the peak at $1560 \mathrm{~cm}^{-1}$ in the FT-IR spectrum of MSNPs- $\mathrm{NH}_{2}$ clearly indicates the presence of $\mathrm{NH}_{2}$ groups on the surface of nanoparticles. But, no such peak observed in the FT-IR spectrum of MSNPs- $\mathrm{PPh}_{3}$ means that no obvious amine groups remained on the surface of $\mathrm{MSNPs}-\mathrm{PPh}_{3}$, further indicating the successful reaction between amine groups and carboxylic acid groups. In addition, one clear peak at $700 \mathrm{~cm}^{-1}$ in the FT-IR spectrum of $\mathrm{MSNPs}^{-\mathrm{PPh}_{3}}$ is in accordance with the deformation vibration of carbon-hydrogen bonds in the phenyl groups, also supporting the functionalization of TPP groups on the surface. Apart from FT-IR spectra, Zeta potential of 
the nanoparticle surface charge was measured in phosphate-buffered saline (PBS) to further confirm the TPP functionalization (Figure 4a). In different protonation/deprotonation environments, the amine groups on the surface of MSNPs- $\mathrm{NH}_{2}$ showed positive surface charge under acidic conditions and negative charges under basic conditions. However, the surface charge of MSNPs- $\mathrm{PPh}_{3}$ was positive under both acidic and basic conditions, indicating that the amine groups were significantly consumed during the amidation. As such, the reaction between amine groups and TPP groups to afford the TPP surface functionality was successfully achieved. In addition, high Brunauer-Emmett-Teller (BET) surface area of $761.585 \mathrm{~m}^{2} / \mathrm{g}$ for MSNPs- $\mathrm{PPh}_{3}$ was observed from the $\mathrm{N}_{2}$ adsorption/desorption measurement. MCM-41 type mesoporous structure of MSNPs- $\mathrm{PPh}_{3}$ was confirmed by the obtained type IV BET isotherm curve, similar to the results obtained in Chapter 1.

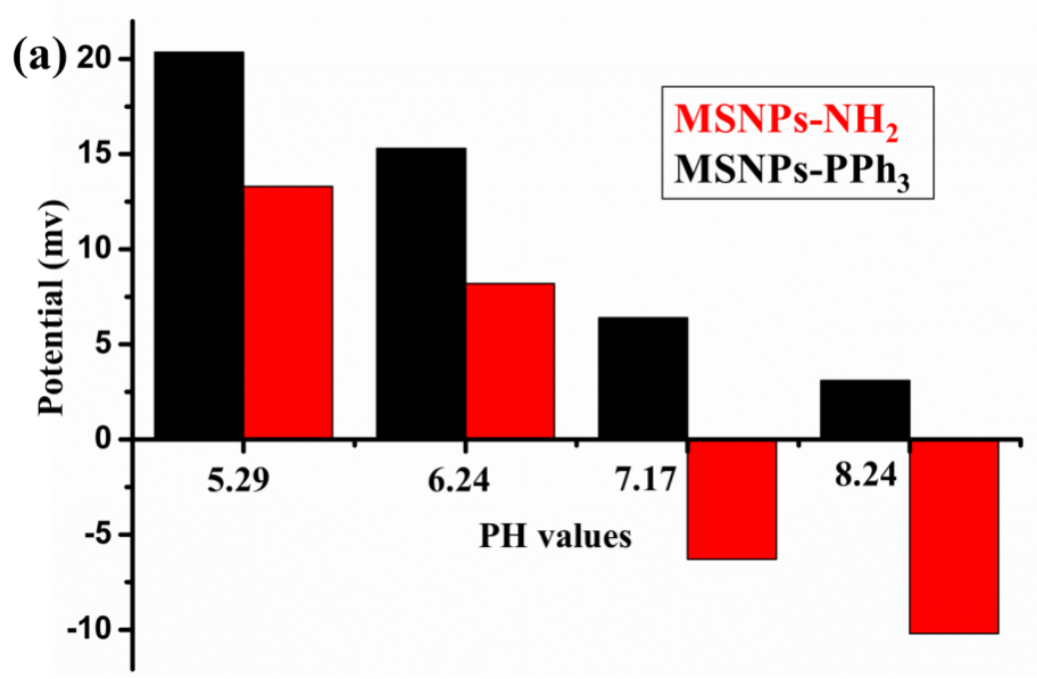




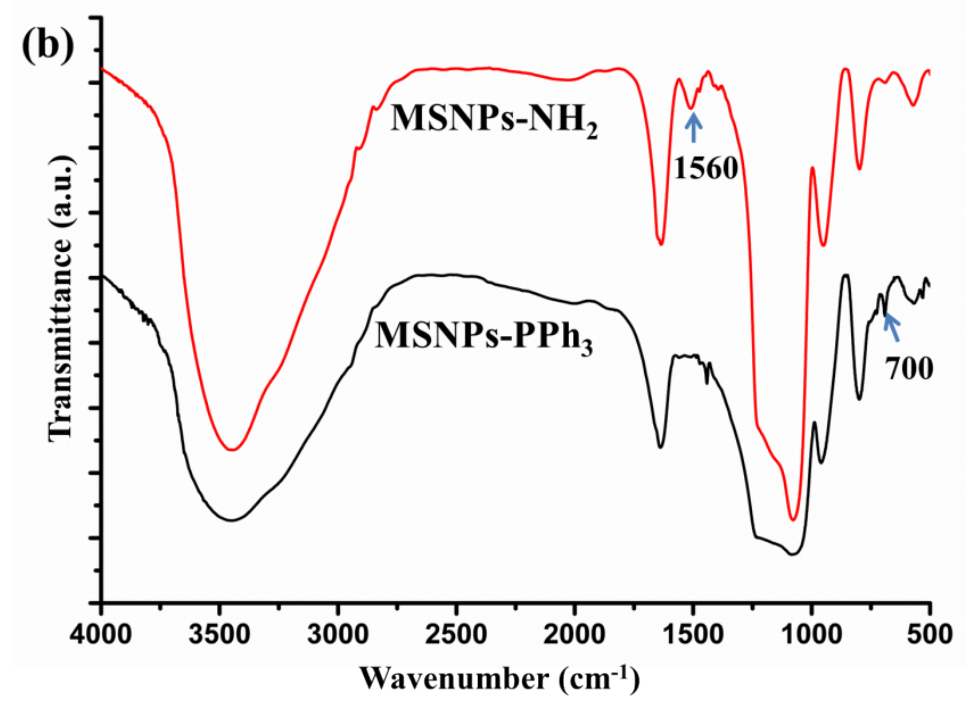

Figure4.(a) Surface charges for MSNPs- $\mathrm{NH}_{2}$ and $\mathrm{MSNPs}-\mathrm{PPh}_{3}$ suspended in $\mathrm{PBS}$ with different $\mathrm{pH}$ values.(b) FT-IR spectra of fabricated MSNPs-NH $\mathrm{H}_{2}$ and $\mathrm{MSNPs}-\mathrm{PPh}_{3}$.

\subsection{2 $\alpha$-TOS loading and intracellular uptake of MSNPs}

(a)

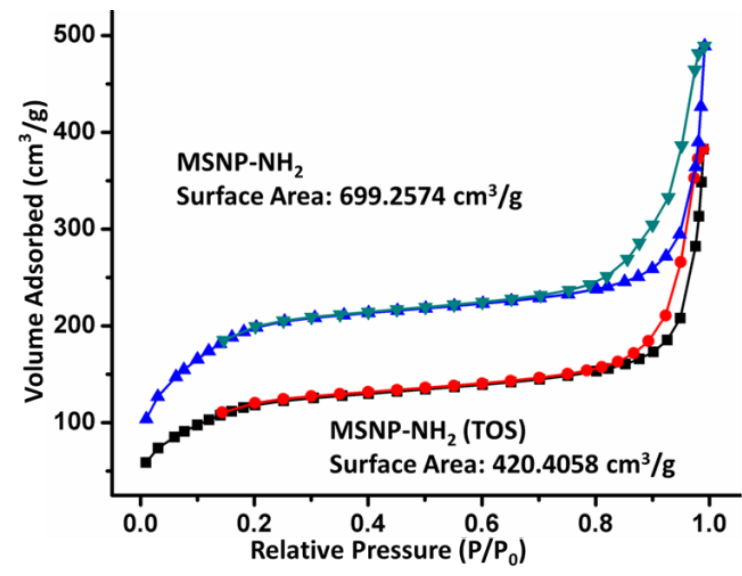

(b)

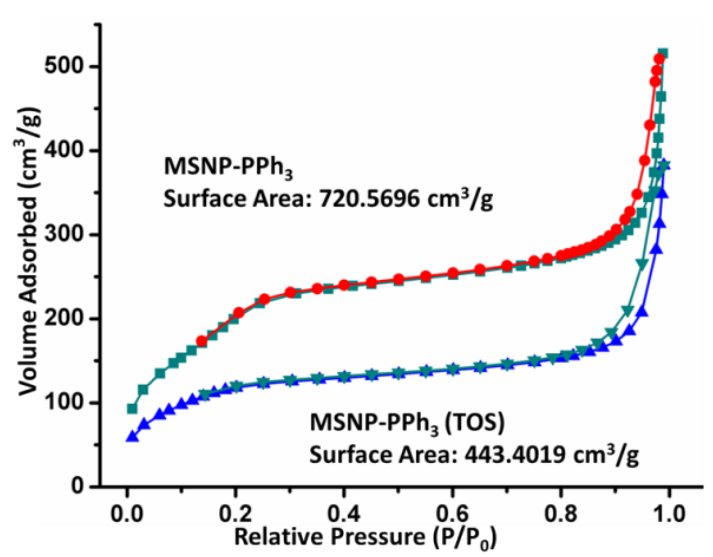

Figure5.BET surface areas of (a) $\mathrm{MSNPs}-\mathrm{NH}_{2}$ and (b) $\mathrm{MSNPs}-\mathrm{PPh}_{3}$ before and after the $\alpha$ TOS loading.

The loading of $\alpha$-TOS was conducted by suspending synthesized nanoparticles (MSNPs$\mathrm{NH}_{2}$ and MSNPs-PPh 3 ) in $\alpha$-TOS solution ( $1 \mathrm{mg} / \mathrm{mL}$ in $95 \%$ ethanol). The drug loading capacities are $3.73 \pm 0.05 \mathrm{wt} \%$ and $3.65 \pm 0.05 \mathrm{wt} \%$ and the entrapmentefficiencies of $\alpha-T O S$ in MSNPs-NH ${ }_{2}$ and MSNPs-PPh 3 were $7.46 \%$ and $7.30 \%$ for MSNPs-NH ${ }_{2}$ and $\mathrm{MSNPs} \mathrm{PPh}_{3}$ respectively, indicating no obvious influence of the TPP surface functionalization on the drug loading capacity of the nanoparticles. The loading capacity was calculated based on the UV 
intensity difference at $284 \mathrm{~nm}$ between stock $\alpha$-TOS solution and supernatant solution after the drug loading. In addition, the successful $\alpha$-TOS loading was characterized by the BET surface area study (Figure 5). Lowered surface areas of MSNPs-NH $\mathrm{N}_{2}(\alpha-\mathrm{TOS})$ and MSNPs$\mathrm{PPh}_{3}(\alpha$-TOS $)$ compared with original MSNPs were observed due to the $\alpha$-TOS loading in the pores of MSNPs. Beyond that, the in vitro $\alpha$-TOS releasing profile from $\mathrm{MSNPs}^{-\mathrm{PPh}_{3}(\alpha-}$ TOS) was investigated (Figure 6), which showed that loaded $\alpha$-TOS drug molecules could be released on time.

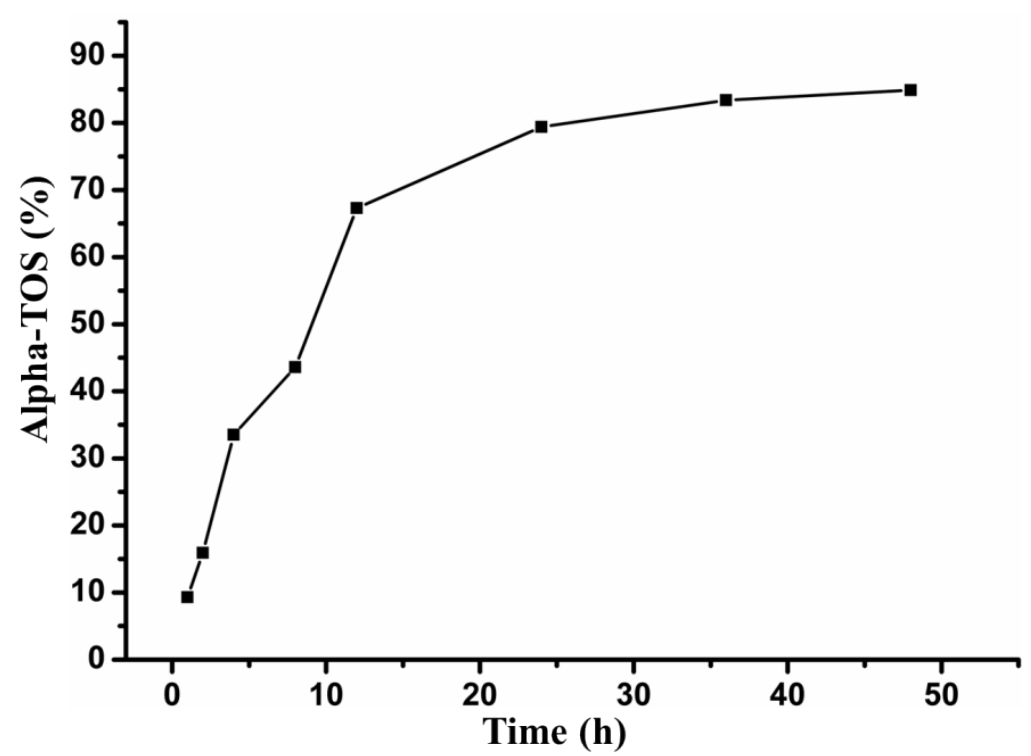

Figure 6. Release profile of MSNPs-PPh $3(\alpha-\mathrm{TOS})$.

The prerequisite of a proper drug delivery carrier is its good intracellular uptake. Thus, intracellular uptake test of MSNPs-NH 2 and $\mathrm{MSNPs}-\mathrm{PPh}_{3}$ with the FITC functionality was performed by applying flow cytometry method using HeLa cervical cancer cell line and HepG2 liver hepatocellular carcinoma cell line (Figure 7). As shown in both $\mathrm{MSNPs}-\mathrm{NH}_{2}$ FITC and MSNPs-PPh ${ }_{3}$-FITC showed high FITC fluorescence intensity in HeLa and HepG2 cells, indicating their successful intracellular uptake as well as good biocompatibility as drug delivery platforms. 

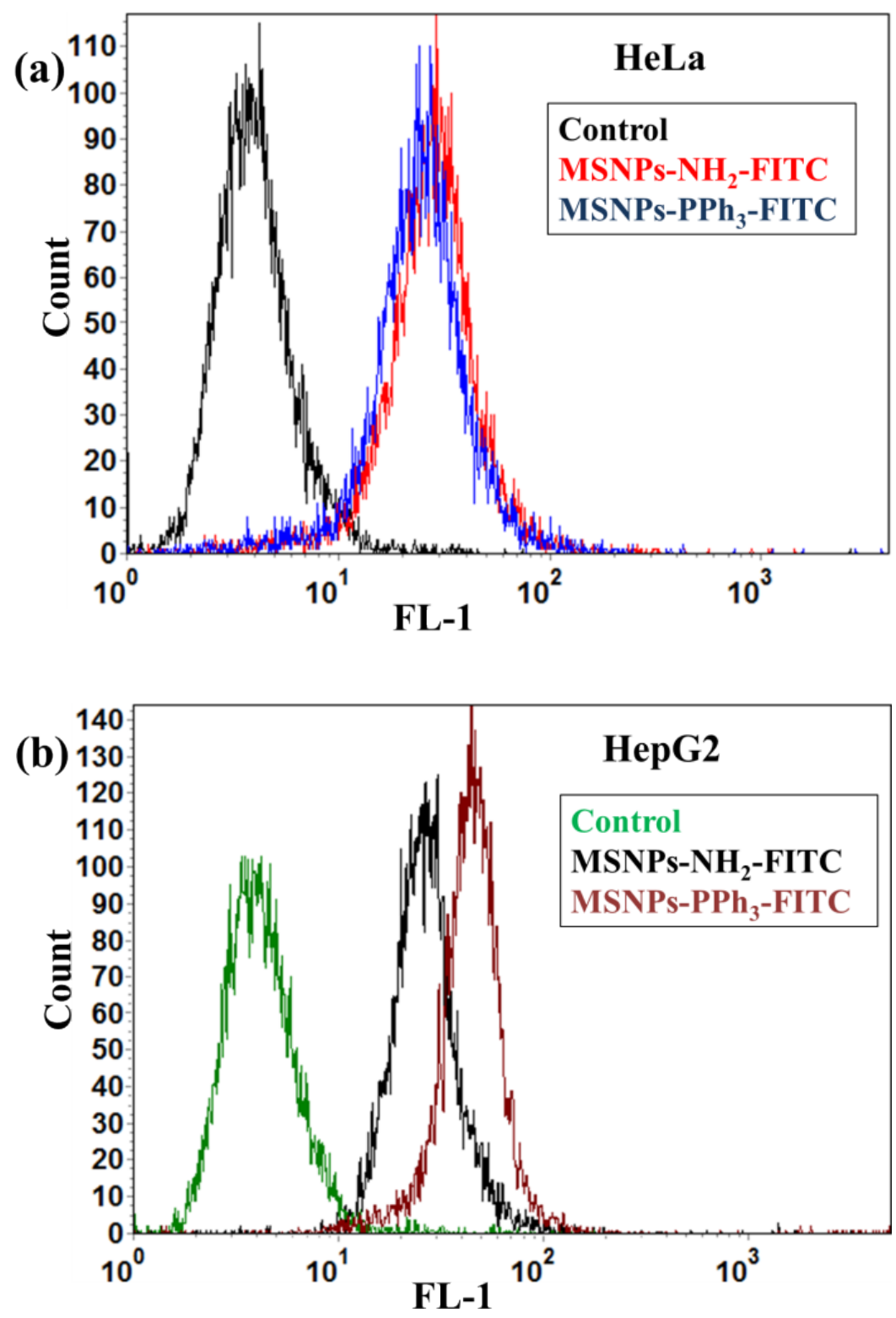

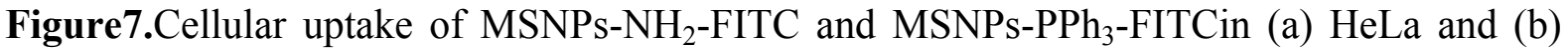
HepG2 cells.FL-1: FITC fluorescence.

So far, sufficient research has shown that MSNPs could be intracellularly internalized through the process of endocytosis in mammalian cells. In order to further investigate the internalization of MSNPs-NH 2 and $\mathrm{MSNPs}-\mathrm{PPh}_{3}$, the endocytosis process after the nanoparticle uptake was studied (Figure 8). It is believed that MSNPs would be initially trapped by lysosomes after the cellular uptake, and such trapping could influence the delivery efficiency. Therefore, time-dependent endocytosis study of MSNPs was conducted using 
confocal laser scanning microscopy with the staining of lysosomes by Lyso-Tracker Red in HepG2 cells

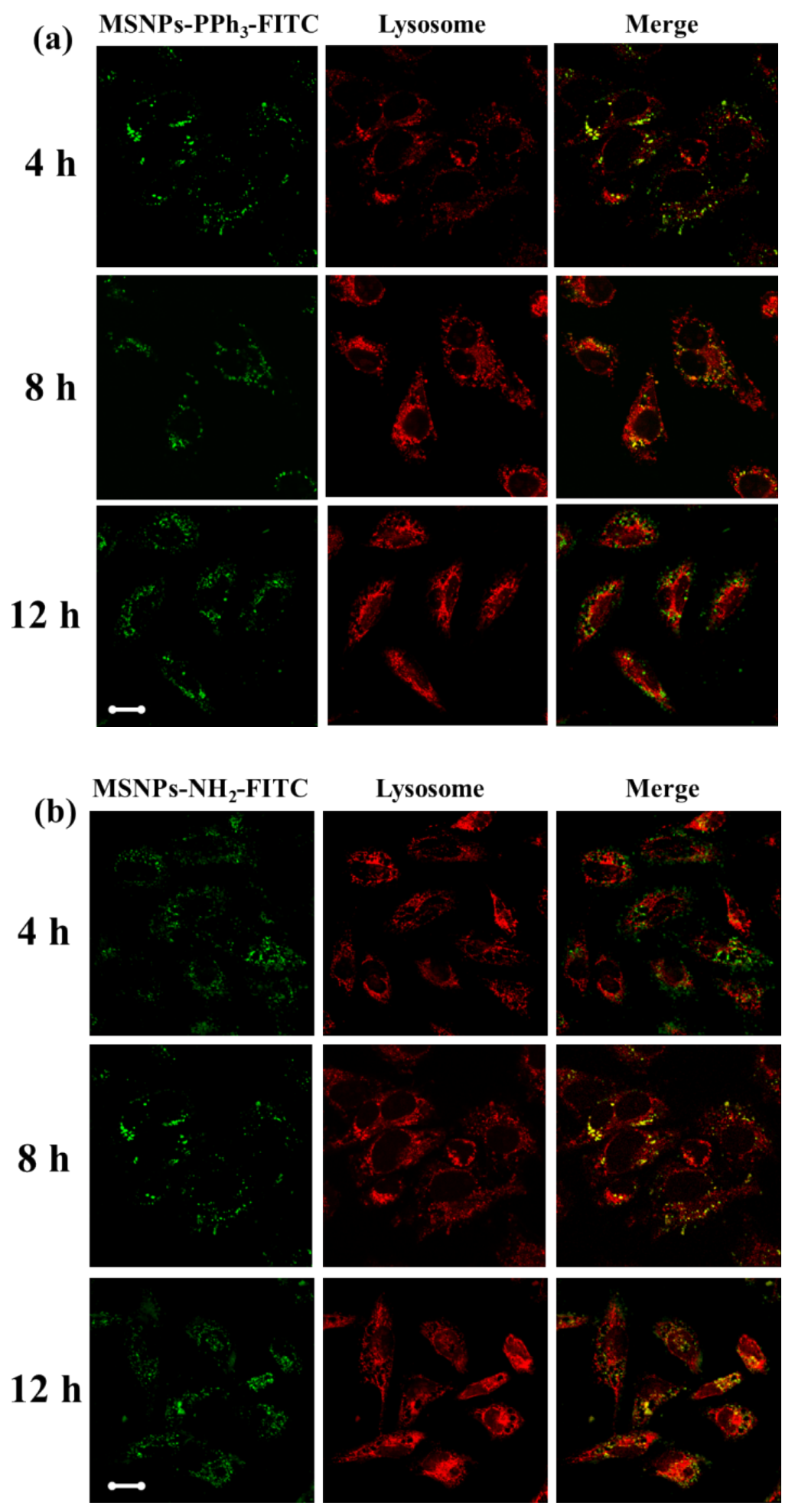

Figure8.Confocal microscope images of HepG2 cells after being treated with (a) MSNP$\mathrm{PPh}_{3}$-FITC $(50 \mu \mathrm{g} / \mathrm{mL})$ and (b) MSNP-NH ${ }_{2}$-FITC $(50 \mu \mathrm{g} / \mathrm{mL})$ for $4 \mathrm{~h}, 8 \mathrm{~h}$ and $12 \mathrm{~h}$ of incubation. Lysosomes were stained by Lyso-Tracker Red $(50 \mathrm{nM})$. Scar bar: $20 \mu \mathrm{m}$. 
According to the obtained images after initial $4 \mathrm{~h}$ incubation, clear green spots corresponding to the fluorescence of the FITC functionality on the surface of MSNPs$\mathrm{NH}_{2}$-FITC and MSNPs-PPh 3 -FITC could be observed, further indicating their successful intracellular uptake and good biocompatibility. In addition, no clear yellow spots appeared in the merged images, meaning no overlap between these MSNPs and lysosomes at this stage. Thus, these MSNPs were not yet trapped by lysosomes after the incubation of initial $4 \mathrm{~h}$. After $8 \mathrm{~h}$ incubation, obvious yellow spots appeared in the cases of MSNPs-NH $2-$ FITC and MSNPs-PPh ${ }_{3}$-FITC, indicating that these MSNPs and lysosomes were co-localized and these MSNPs were trapped by lysosomes. After further incubation, however, no clear yellow color could be observed in the case of MSNPs-PPh 3 -FITC, proving that MSNPs-PPh - FITC could escape from lysosomes at the time of $12 \mathrm{~h}$ incubationSuch lysosome escape can be possibly attributed to the facilitation of cross membrane process due to the TPP surface functionality of MSNPs-PPh 3 . For the case of MSNPs-NH2-FITC after $12 \mathrm{~h}$, distinct yellow colorcould still be observed, meaning that MSNPs-NH$-\mathrm{NH}_{2}$-FITC was trapped by lysosomes as before. Therefore, it could be concluded that MSNPs-PPh ${ }_{3}$ inducedrapid endocytosis. Similar results were also obtained by using HeLa cells in Chapter 1.

\subsubsection{Mitochondria targeting study}

The study of mitochondria targeting by MSNPs- $\mathrm{PPh}_{3}$ was carried out using confocal laser microscopy. Different cancer cell lines (HeLa and HepG2 cell lines) were treated with MSNPs-PPh ${ }_{3}$-FITC, and mitochondria within cells were stained by Mito-Tracker Red (Invitrogen). As seen from the obtained confocal laser microscopy images, these MSNPs and mitochondria were indicated as green and red dots, respectively (Figure 9). 
MSNPs-NH - FITC

(a)

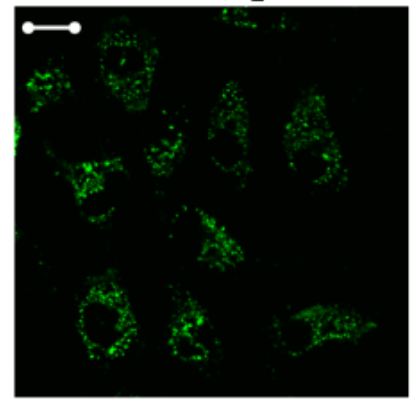

Mitochondria

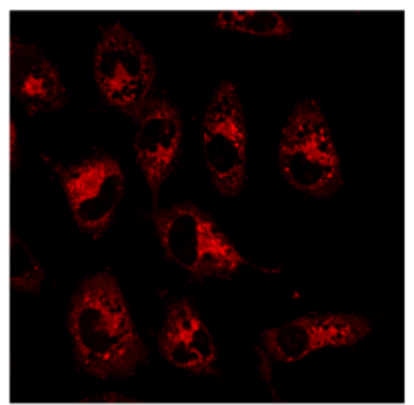

MSNPs-PPh ${ }_{3}$-FITC
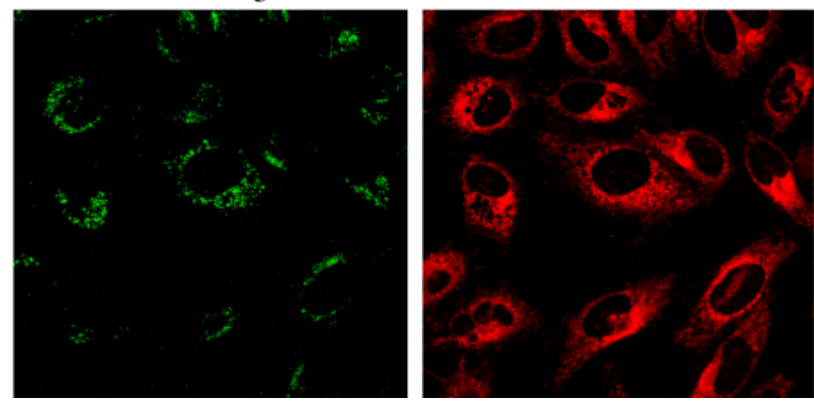

MSNPs-NH - -FITC

(b)

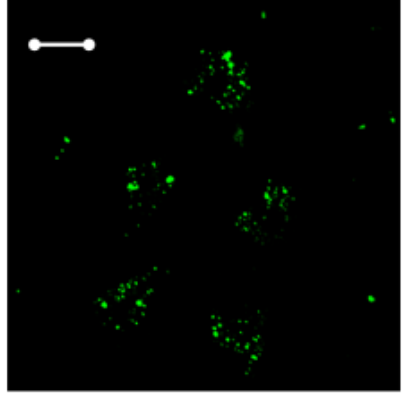

MSNPs-PPh -FITC

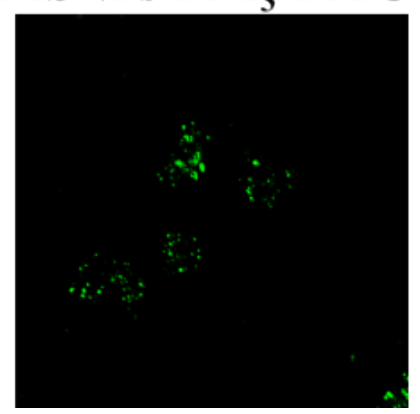

Merge
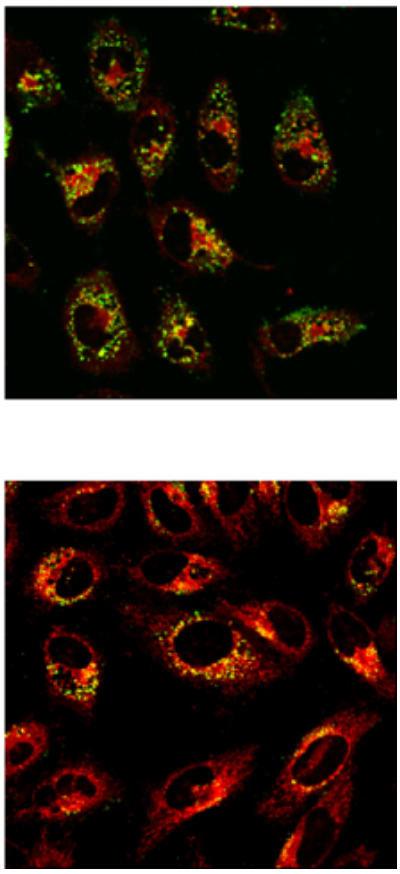

Merge
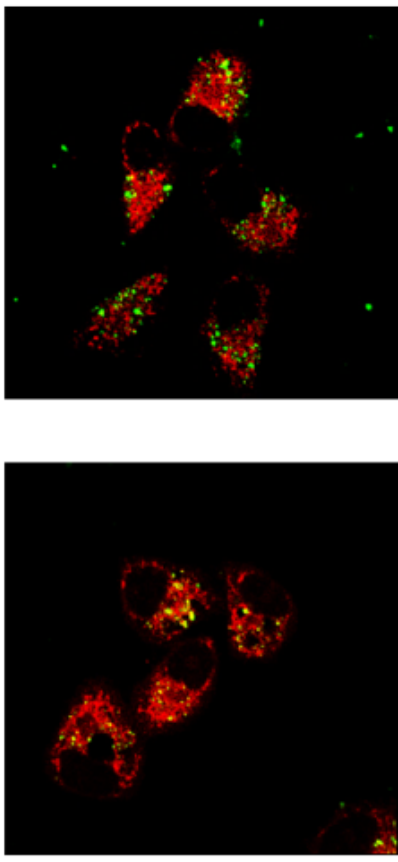

Figure9.Confocal microscope images of (a) HeLa cells and (b) HepG2 cells after being

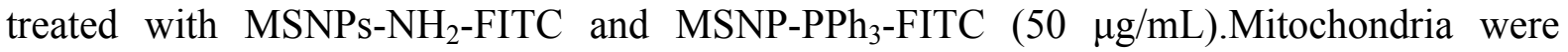
stained by Mito-Tracker Red (50 nM). Scar bar: $20 \mu \mathrm{m}$.

In the case of treating HepG2 cells with MSNPs-NH $\mathrm{N}_{2}$-FITC, no obvious yellow dots representing the co-localization of green and red dots could be found in the merged image. In contrary, distinct yellow dots could be observed in the merge image when using MSNPs- 
$\mathrm{PPh}_{3}$-FITC, indicating the successful co-localization of $\mathrm{MSNPs}-\mathrm{PPh}_{3}$-FITC and mitochondria, and further proving the evident ability of $\mathrm{MSNPs} \mathrm{PPh}_{3}$ in targeting mitochondria of HepG2 cells. Similar mitochondria targeting effect of MSNPs- $\mathrm{PPh}_{3}$ could be found in HeLa cells as well. The overlap coefficient indexes of Figure 9were calculated as well. The indexes for merge images in Figure 9a were 0.408 for MSNPs-NH $2-F I T C$ and 0.662 for $\mathrm{MSNPs}-\mathrm{PPh}_{3}$-FITC, indicating that MSNPs with TPP functionalization presented good overlap effect between MSNPs and mitochondria.
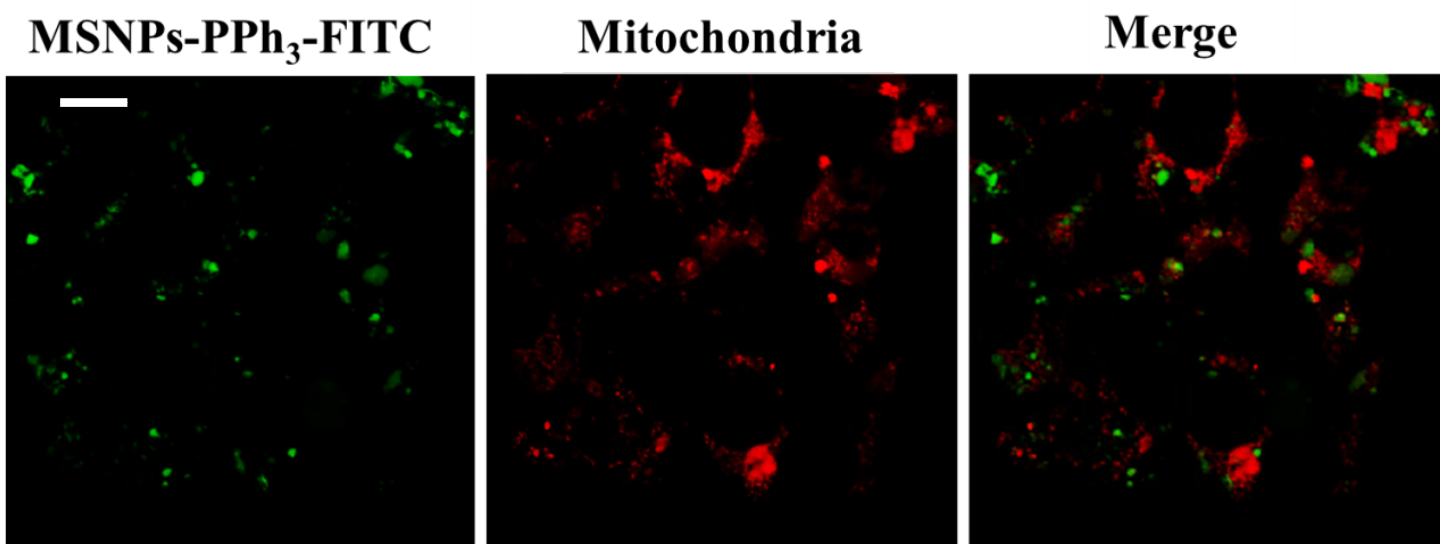

Figure10.Confocal images of HEK293 cells after the treatment of MSNPs-PPh ${ }_{3}$-FITC. Scale bar: $20 \mu \mathrm{m}$.

Interestingly, when treating HEK293 normal cells with MSNPs-PPh ${ }_{3}$-FITC, no obvious colocalization could be observed (Figure 10). Such observation could be probably due to different mitochondria membrane activity and potential, since mitochondria in cancer cells usually have higher activity and membrane potential than normal cells. Furthermore, it was found that mitochondria in normal cells could not be well stained as the staining of mitochondria is based on highly negatively charged membrane potential, indicating that mitochondria in normal cells were less active than that in cancerous cells.

To further validate the successful targeting to mitochondria in cancer cells, the fluorescence and UV detections of isolated mitochondria from treated HeLa, HepG2, and HEK293 cells were conducted (Figure 11). Mitochondria were isolated from cells after the treatment of 
different MSNPs. As shown in the result, higher FITC fluorescence intensity was observed in the isolated mitochondria population from both HeLa and HepG2 cells treated with MSNPs$\mathrm{PPh}_{3}$-FITC, directly proving the mitochondria targeting ability of MSNPs-PPh 3 . In the case of mitochondria from HEK293 normal cells, however, no obvious FITC intensity difference was found, showing no specific mitochondria targeting effect of MSNPs-PPh ${ }_{3}$ in $\mathrm{HEK} 293$ cells. Apart from the fluorescence detection, the UV intensity detection was also performed, since the loaded $\alpha$-TOS has a strong UV absorption peak at $284 \mathrm{~nm}$. Here, the UV test was conducted after cells were treated with $\alpha$-TOS loaded MSNPs. As seen from the result, obviously higher UV intensity in mitochondria was found in the cases of HeLa and HepG2 cancer cells, and no obvious UV intensity difference was observed from HEK293 normal cells. Thus, it could be concluded that MSNPs- $\mathrm{PPh}_{3}$ has an excellent capability in targeting mitochondria of cancer cells.
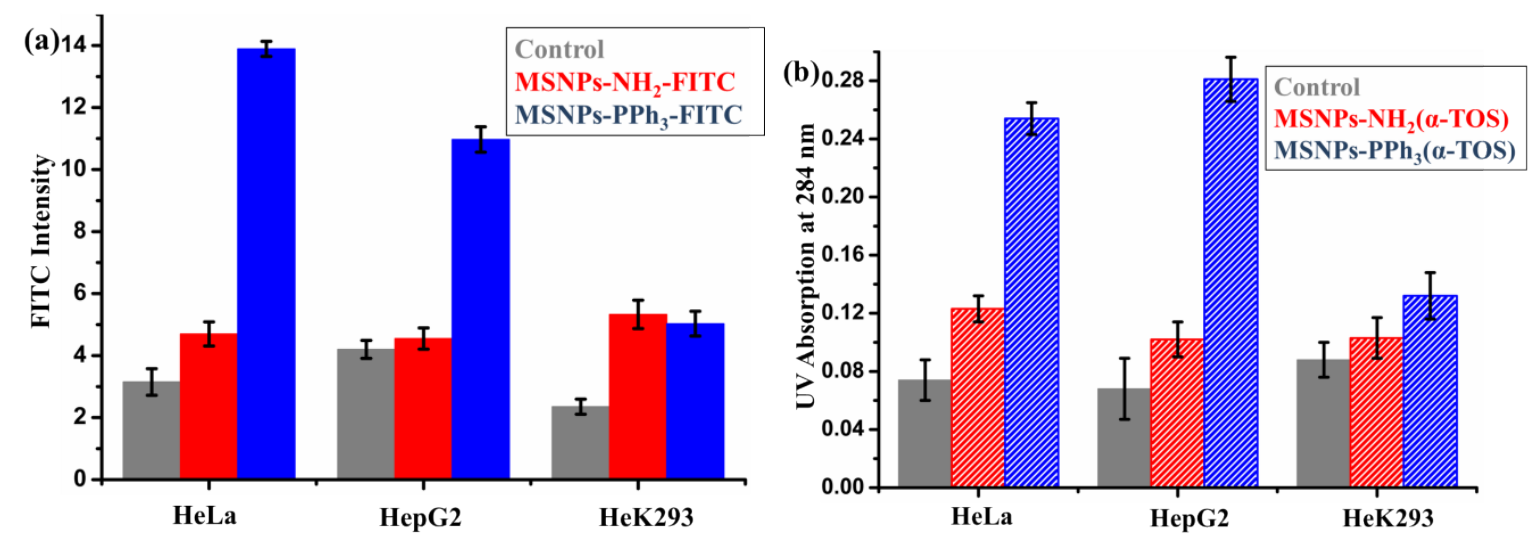

Figure11.(a) FITC fluorescence and (b) UV absorption intensities of isolated mitochondria originated from HeLa, HepG2 and HEK293 cells without and with indicated treatments.

\subsubsection{Cytotoxicity and mitochondria functional alterations}

In order to investigate the anticancer efficacy of drug loaded MSNPs as well as the biocompatibility of MSNP-based drug delivery platforms, MTT assay was carried out in HeLa and HepG2 cancerous cells as well as HeK293 normal cells. Higher intensity of MTT assay (cell survival ratio) indicates higher cellular activity and lower cytotoxicity. 
From the obtained data (Figure 12), it could be noted that all tested cells showed high cell viability (above $80 \%$ ) even at $100-200 \mathrm{mg} / \mathrm{mL}$ concentrations of $\mathrm{MSNPs}-\mathrm{NH}_{2}$ and MSNPs-PPh 3 , meaning that these MSNP-based nanocarriers have good biocompatibility toward both cancerous cells and normal cells. Having loaded drugs, MSNPs-PPh 3 ( $\alpha$-TOS) presented high anticancer efficiency at concentrations from 25 to $200 \mathrm{mg} / \mathrm{mL}$ in both HeLa and HepG2 cancerous cells, since cell survival ratios in this concentration range were much lower than that in the case of $\mathrm{MSNPs}_{-} \mathrm{NH}_{2}(\alpha-$ TOS). This observation can be reasonably explained by the fact that $\alpha$-TOS is a kind of drug particularly effective to mitochondria and such active effect can be enhanced by targeted delivery of $\alpha$-TOS to mitochondria using the MSNPs- $\mathrm{PPh}_{3}$ nanocarrier, increasing the concentration of $\alpha$-TOS in mitochondria at the same time. For the test in HEK293 normal cells, however, high cell viability was still obtained in the whole range of concentrations, indicating that both MSNP nanocarriers and $\alpha$-TOS loaded MSNPs showed no obvious cytotoxicity toward normal cells. Therefore, it could be concluded that the anticancer efficacy of $\alpha$-TOS can be significantly enhanced by mitochondria targeted MSNPs- $\mathrm{PPh}_{3}$, and more importantly, $\alpha$-TOS loaded MSNPs$\mathrm{PPh}_{3}$ showed no obvious cytotoxicity to normal cells.

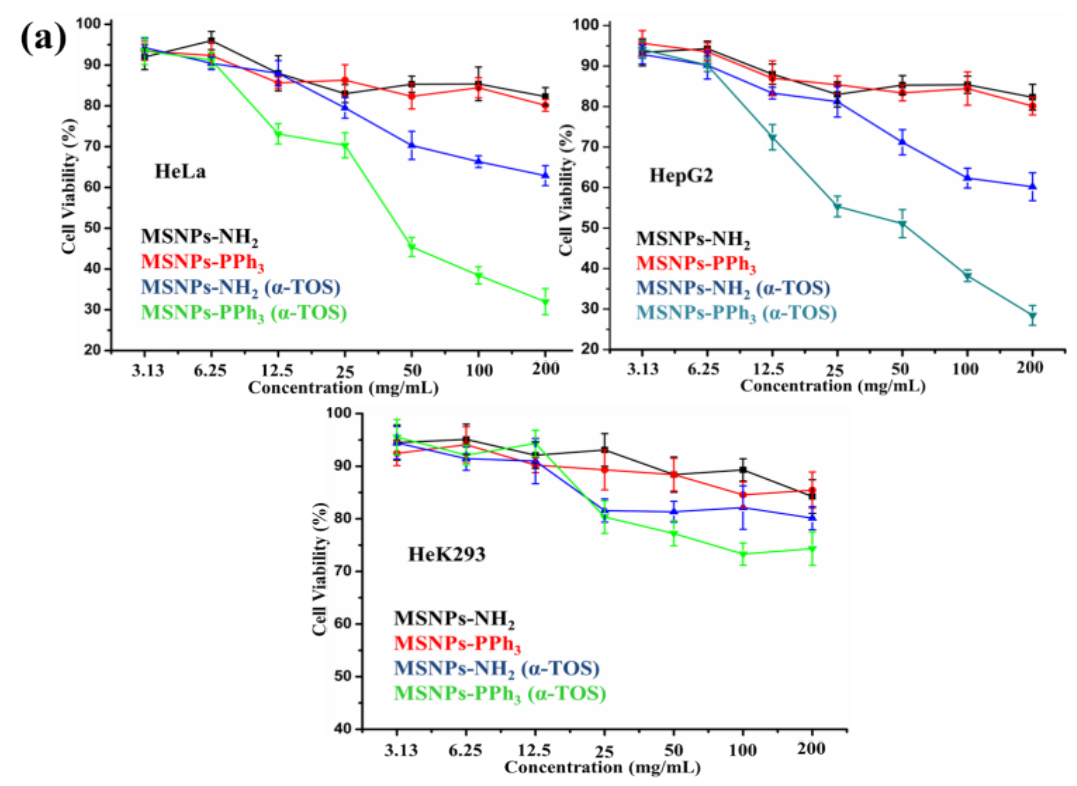




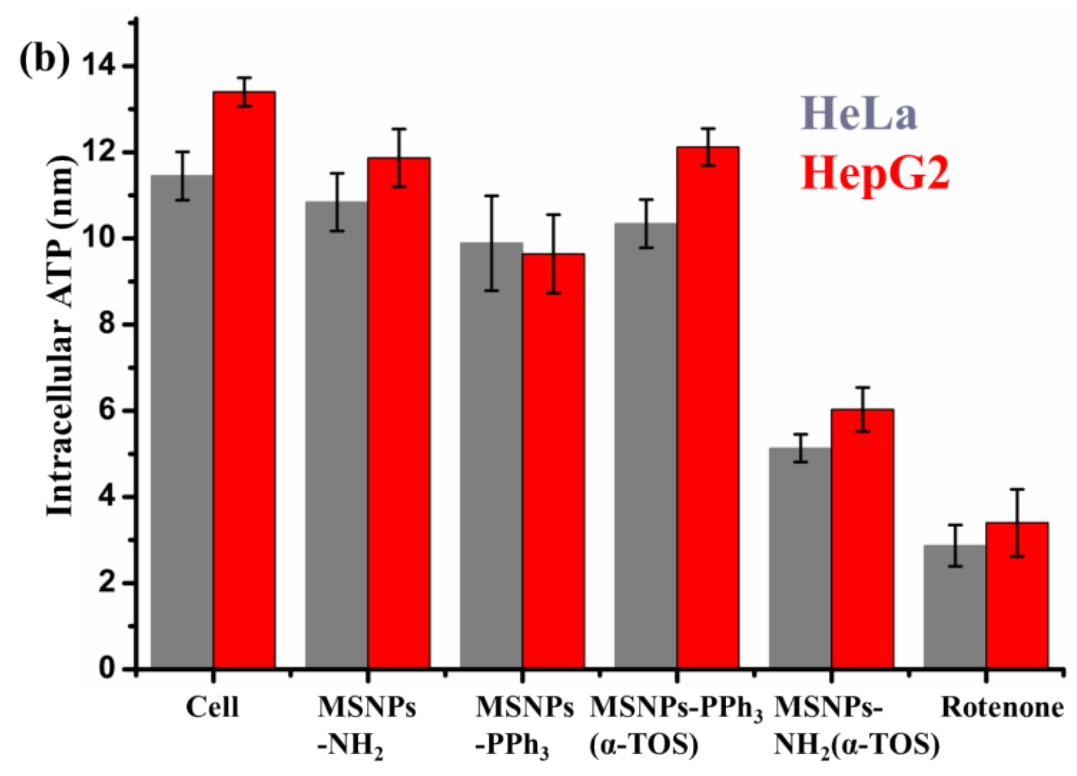

Figure12.(a) Cells viability of HeLa, HepG2 and HEK293 cells and (b) intracellular ATP level of HeLa and HepG2 cells after the treatment of different MSNPs $\left(\mathrm{MSNPs}_{2} \mathrm{NH}_{2}\right.$, MSNPs-PPh, MSNPs-PPh 3 ( $\alpha$-TOS), and MSNPs-NH $\left.\mathrm{NH}_{2}(\alpha-\mathrm{TOS})\right)$ for $24 \mathrm{~h}$.

Since $\alpha$-TOS has the target site in mitochondria, the function of mitochondria should be affected when the $\alpha$-TOS drug is delivered into mitochondria. Therefore, alterations of mitochondria resulted from delivered $\alpha$-TOS were investigated. The distinct function of mitochondria is the ATP production, which is the most commonly used form of energy to supply cellular activities. In cancerous cells, the energy supply is more important than that in normal cells because of the long lasting cell proliferation of cancerous cells, leading to the fact that cancerous cells are more sensitive toward cellular ATP level alteration. As such, we investigated intracellular ATP levels before and after the treatment of different MSNPs. The relative results obtained were calculated based on a standard curve of ATP solutions with various concentrations. Rotenone was used as the positive control. As shown in the result, in the case of testing MSNPs without the $\alpha$-TOS loading, no obvious decrease of intracellular ATP level was observed, further demonstrating the good biocompatibility of MSNP-based nanocarriers. Interestingly, more significant decrease of the ATP level was obtained in 
the case of MSNPs-PPh 3 ( $\alpha$-TOS) than that of MSNPs-NH $\mathrm{NH}_{2}(\alpha-\mathrm{TOS})$, which could be resulted from the successful delivery of $\alpha$-TOS to mitochondria by $\mathrm{MSNPs}-\mathrm{PPh}_{3}$. Thus, the active $\alpha$-TOS drug was successfully delivered into mitochondria by MSNPs$\mathrm{PPh}_{3}$ for reducing intracellular ATP level.
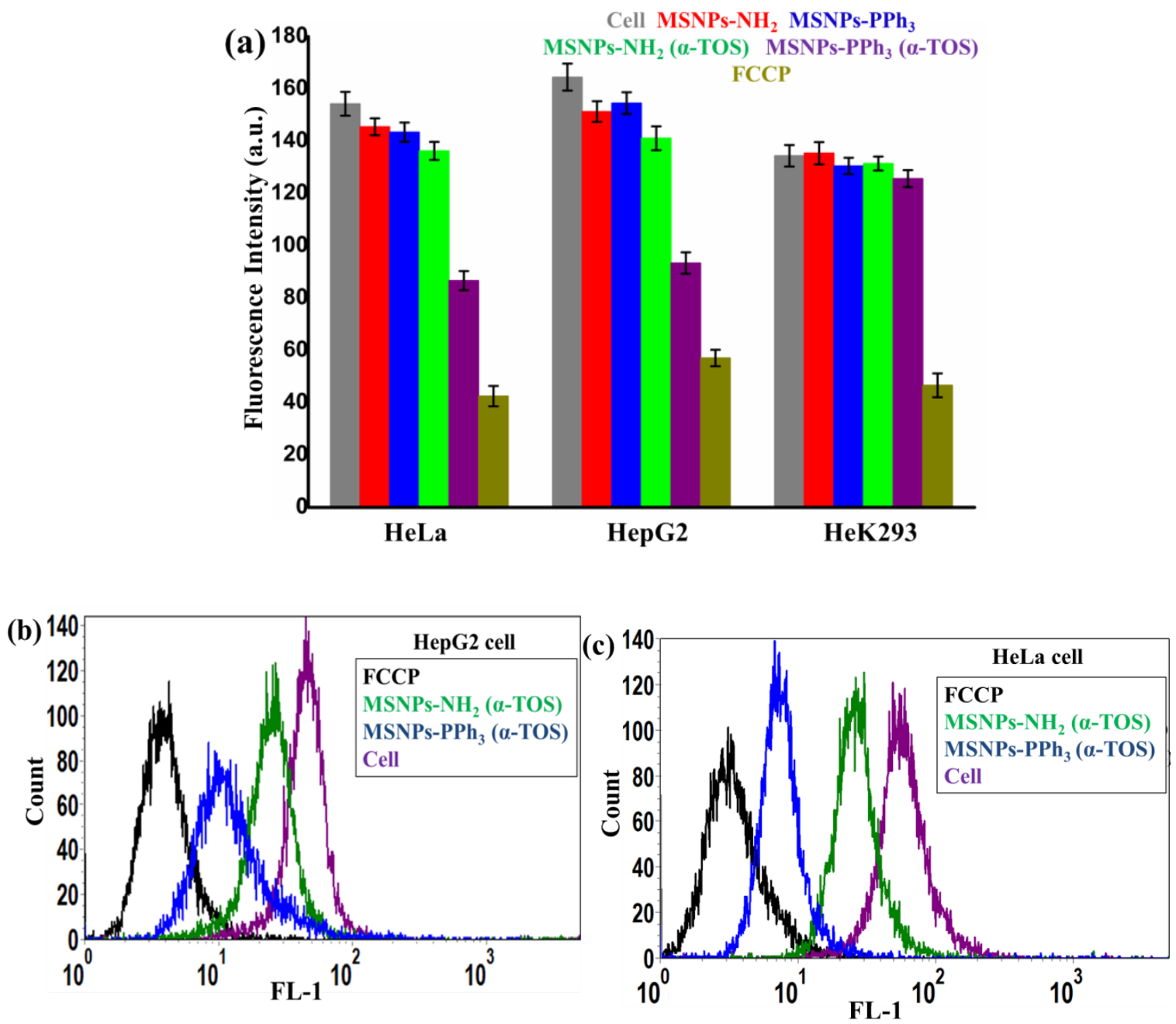

Figure 13. (a) Mitochondria membrane potential (TMRE fluorescence intensity) detected after treating HeLa cells with FCCP and different MSNPs $(50 \mu \mathrm{g} / \mathrm{mL})$ for 24 h. (b) (c) Flow cytometry studies of mitochondria membrane potential. FL-1: Fluorescence of TMRE.

One of the important features of mitochondria is highly negatively charged membrane that gives high mitochondria membrane potential for controlling the ion transportation, metabolism as well as cell death. Such high membrane potential, on the other hand, is the symbol of active mitochondria. Thus, the detection of mitochondria membrane potential was 
conducted in HeLa and HepG2 cells in order to measure the activity of mitochondria after the treatment of MSNPs-PPh $(\alpha-\mathrm{TOS})$ (Figure 13). Active mitochondria with high membrane potential could be stained by an experimental TMRE kit, giving strong fluorescence intensity. From the results, compared with control cells, no obvious influence on the mitochondria membrane potential was observed when treating cells with MSNPs- $\mathrm{NH}_{2}$ and $\mathrm{MSNPs}-\mathrm{PPh}_{3}$, again showing the good biocompatibility of these MSNPs. While in the event of testing MSNPs loaded with $\alpha$-TOS, lowered mitochondria membrane potential of both HeLa and HepG2 cells treated with MSNPs-PPh 3 ( $\alpha$-TOS) was observed than that with MSNPs-NH $\mathrm{NH}_{2}(\alpha-$ TOS), indicating that the function of $\alpha$-TOS was achieved by using mitochondria targeted MSNPs-PPh ${ }_{3}$. In other words, $\alpha$-TOS drug could be successfully delivered to mitochondria by MSNPs- $\mathrm{PPh}_{3}$, resulting in the accumulated concentration of $\alpha$-TOS in mitochondria as well as the lowered mitochondria membrane potential.

\subsubsection{Apoptosis study}

Apoptosis is a biomedical event that occurs in the process of programmed cell death in multicellular organisms, which can lead to various cellular changes in physical morphology, nuclear fragmentation, global mRNA decay and so on. It is essential to know that the apoptosis process is highly regulated, since it is unstoppable in both cancerous and normal cells. Thus, it is generally accepted that inducing the apoptosis in cancerous cells is one of the methods to achieve the cancer treatment. More importantly, the cascade apoptosis process is closely related to the function of mitochondria. For example, it was proven that the primary signal molecule of apoptosis process, cytochrome $\mathrm{c}$, is released from mitochondria because of the formation of mitochondrial apoptosis-induced channel. It was also proven that $\alpha$-TOS is an effective apoptosis inducer. Therefore, it is necessary to investigate the apoptosis caused by the dysfunction of mitochondria that is resulted from $\alpha$-TOS delivered by $\mathrm{MSNPs}-\mathrm{PPh}_{3}$. 
During the cascade apoptosis process, featured measurements commonly involve the activity detections of caspase 9 that is one of the initiator caspases, and caspase 3 that is activated by caspase 9 subsequently.
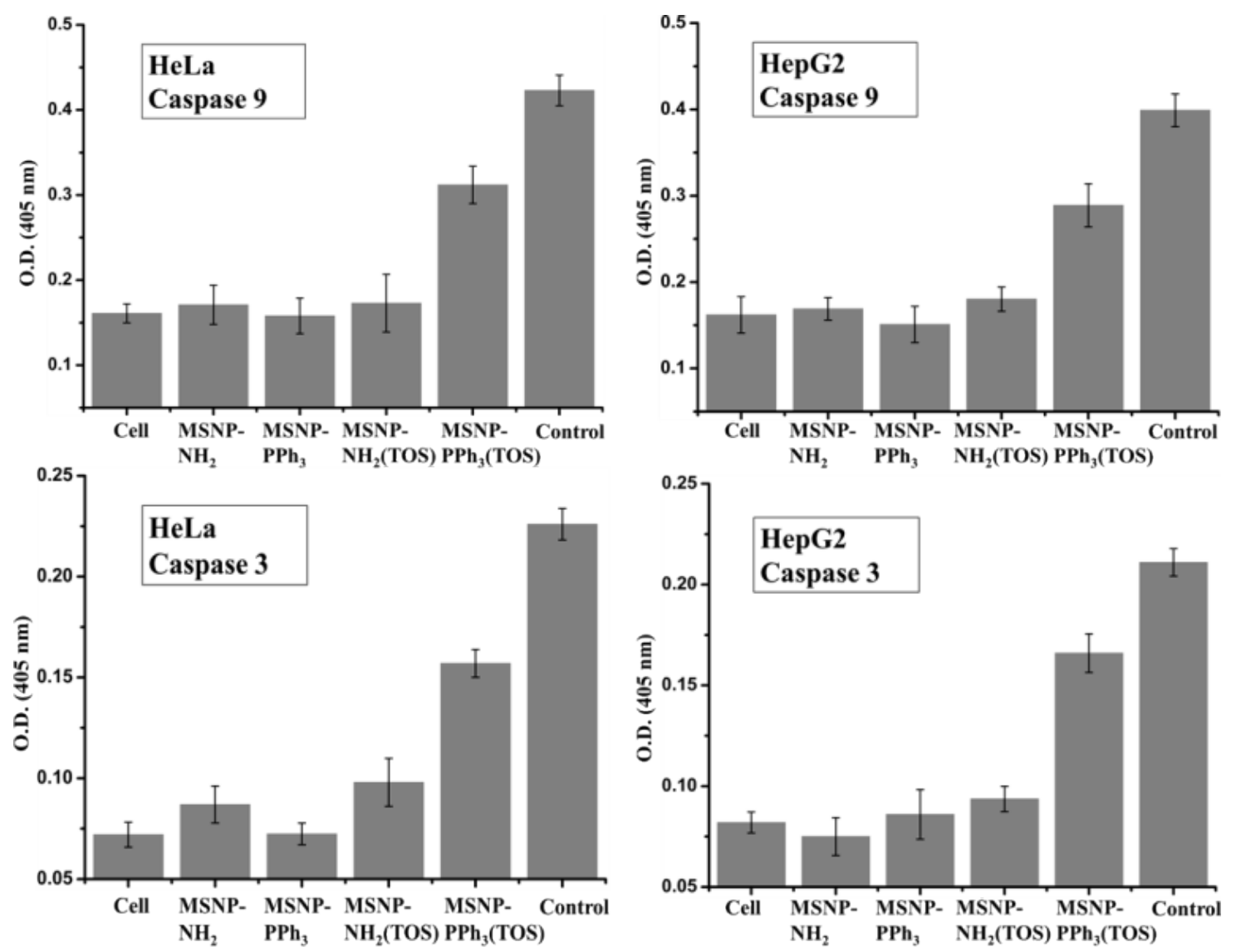

Figure 14.Activity of caspase 9 and caspase 3 in HeLa and HepG2 cells after the treatment of different MSNPs for $24 \mathrm{~h}$. Control: FCCP.O.D.: optical density.

Here, the activities of caspase 9 and caspase 3 were detected in HeLa and HepG2 cancerous cells after the treatment of different MSNPs (Figure 14). FCCP was set as a positive control. Higher optical intensity at $405 \mathrm{~nm}$ corresponds to higher activity of caspase. As shown in Figure 14, the treatment by MSNPs- $\mathrm{NH}_{2}$ and $\mathrm{MSNPs}-\mathrm{PPh}_{3}$ showed almost no influence on activities of both caspase 9 and caspase 3 in HeLa and HepG2 cells, further proving the biocompatibility of these MSNPs. In the case of testing $\alpha$-TOS loaded MSNPs in HeLa and HepG2 cells, however, MSNPs-PPh 3 ( $\alpha$-TOS) presented higher activities of both caspase 9 and caspase 3, indicating higher activity of apoptosis process induced by $\mathrm{MSNPs} \mathrm{PPh}_{3}(\alpha-$ 
TOS). Thus, it could be concluded that the property of hydrophobic $\alpha$-TOS to effectively induce the apoptosis can be enhanced when delivered by MSNPs- $\mathrm{PPh}_{3}$ to mitochondria.

\subsection{Conclusion}

In summary, mitochondria targeted MSNPs $\left(\mathrm{MSNPs}-\mathrm{PPh}_{3}\right)$ were successfully fabricated by the surface functionalization of TPP. The property of targeting mitochondria was demonstrated by CLSM study in HeLa and HepG2 cancerous cell lines. Hydrophobic anticancer drug, $\alpha$-TOS, was successfully loaded in designed MSNPs for the first time. The synthesized MSNPs showed good cellular uptake and biocompatibility against HeLa and HepG2 cells, and quick endocytosis and lysosome escape. More importantly, $\alpha$-TOS loaded MSNPs showed no toxicity to HeK293 normal cells. The hydrophobicity of $\alpha$-TOS was overcome by using the MSNPs-PPh 3 nanocarrier. Anticancer effects of $\alpha$-TOS including inducing the cytotoxicity and mitochondria dysfunctions as well as apoptosis were achieved and further enhanced by the targeted delivery of $\mathrm{MSNPs}-\mathrm{PPh}_{3}$ to mitochondria. As a result, the present research broadens the application of MSNPs as drug delivery platforms, demonstrating its promising potential in overcoming the hydrophobicity of drug molecules as well as targeted mitochondrial sub-cellular drug delivery.

\section{Chapter 4: Targeted Delivery of Doxorubicin to Mitochondria by using}

\section{Mesoporous Silica Nanoparticles for Overcoming Drug Resistance}

\subsection{Introduction}

One of the major challenges in cancer treatment is the encounter of anticancer drug resistance. In general, drug resistance is the reduced effectiveness of one certain drug such as antibiotic, antheimintic or an antineoplastic in treating a disease or condition. As cancer cells 
present infinite cell proliferation, the evolving rate and the mutation rate of cancer cells are much faster than that in normal cells caused by long lasting cell proliferation, resulting in the fact that cancer cells are more capable of adjusting different microenvironments ${ }^{213}$. Hence, in the cancer treatment, the property of drug resistance of cancer cells evolved along with the adjusting process of cancer cells to new environment caused by the introduction of such drug. When cancer cells have adjusted to the concentration of certain drug, they will not be killed by the drug through the original way of action of this drug 214,215 .

The failure of cancers to effectively respond to a specific way of therapy can be mainly resulted from two general considerations: the host factors and drug induced genetic or epigenetic alterations inside such cancer cells ${ }^{216-220}$. Typically, the host factors involve poor tolerance of drug to reach the effective concentration, poor absorption of certain drug molecules, inability to effectively deliver drug molecules to the target site and numerous alterations in the host tumor environment which may affect the interactions between drug molecules and target site.

As the ability of drug resistance in cancer cells is a result of cell evolvement, developing effective method to overcome drug resistance is very challenging and imperative. However, up to date, effective anti-drug resistance methodology has not been developed yet. Along the way of conducting various trials, some important information regarding drug resistance of cancer cells have been discovered. It is found that the most challenging consideration of developing effective anti-drug resistance methodology is that each type of cancer cells has different genetic alterations after being treated in drug containing environment, resulting in various random gene expressions to "fight" with drug molecules. Even within a certain type of cancer cells, different derived cells will possibly exhibit different genetic mutations with respect to a specific anticancer drug treatment. In addition, even though some cancer cells are not originally resistant to a specific drug, the strong selection power imposed by drug 
treatment will promote the overgrowth of drug resistant cancer cells, which may be of small amount though. As a result, developing effective anti-drug resistance system is very challenging. Fortunately, however, the major mechanisms of cancer cells becoming resistant to anticancer drugs have been extensively investigated for over 40 years. Such major mechanisms induce malfunction of specific receptor or transporting molecules on cell membrane, the evolved alterations of specific chemical structure of drug molecules or alterations of target molecules of specific anticancer drug. By knowing these mechanisms, it was proposed that use of different anticancer drug with various target molecules, different intracellular ways of action or different required receptor molecules on cell membrane might allow for effective outcome of overcoming drug resistance and high cure rates. However, due to high mutation rates as well as unlimited cell proliferation, cancer cells are found to be able to utilize various mechanisms of drug resistance that include simultaneous resistance to numerous different anticancer drug with different chemical structures and cellular functions. Such observation is known as multi-drug resistance which can result in cancer cells with the ability to prevent accumulation of drug molecules, through enhancing the efflux of drug molecules or changing the structure of cell membrane such as affecting the amount of $\operatorname{lipids}^{221}$.

Among such discovered mechanisms of drug resistance, the most important one is the enhanced efflux of anticancer drug molecules, which leads to the alterations in pathways of drug molecule uptake and efflux from cancer cells ${ }^{222}$. So far, it has been found that the major mechanism of drug molecule efflux in cultured cancer cells is the expression of an energy dependent drug transporting protein which is known as P-glycoprotein (P-gp). Theexpression of P-gpis the product of multidrug resistant 1 (MDR1) gene, which is the first genome member responsible for expressing a large family of ATP-dependent transporting proteins ${ }^{223}$. In addition, P-gp is widely expressed in various types of human cancers including liver 
cancer, pancreatic cancer, leukemia, kidney and childhood cancers and so on ${ }^{221}$,222. Besides, P-gp is a 170,000-dalton-molecular weight phosphoglycoprotein, which consists of two trans-membrane regions and two ATP binding regions. P-gp is observed to be able to detect and bind numerous anticancer drugs as they enter the cell membrane. These anticancer drugs involve many commonly applied drugs such as doxorubicin (DOX), vinblastine and taxol as well as many types of pharmaceuticals ${ }^{223,224}$. The common working mechanism of P-gp is that after detecting and binding with the target anticancer drug molecules, the ATP binding region in P-gp will hydrolysis one ATP molecule, resulting in the transporting and release of drug molecule out of cancer cells. Then, the second ATP molecule was used to restore the structure of P-gp to maintain the cycle of binding and releasing of drug molecules. By knowing this mechanism, we can find that the ATP energy supply is very crucial in maintaining the function of P-gp. Therefore, it is reasonable to propose that by affecting the energy supply in cancer cells, the function of P-gpwill be inhibited and then drug resistant property may be inhibited as well.

Considering the fact that ATP molecules are mostly generated in mitochondria as mentioned before, mitochondria in drug resistant cancer cells have become the target for overcoming drug resistance. Here in this project, we applied mesoporous silica nanoparticles (MSNPs) to targeted deliver DOX drug molecules into mitochondria, leading to the malfunctions of mitochondria including the lowered ATP production and further the dysfunction of P-gp on cell membrane. Accordingly, the intracellular concentration of DOX molecules was enhanced which results in the apoptosis and death of drug resistant cancer cells. To our best knowledge, this is the first report with regard to applying DOX to overcome DOX resistant cancer cells by altering the target site of DOX by mitochondrial targeted MSNPs. As discussed before, by conjugating of lipophilic triphenylphosphonium (TPP) molecules on the surface of MSNPs, the resulted MSNPs were effective in targeting mitochondria. The malfunctions of 
mitochondria and the cytotoxicity of DOX loaded MSNPs in DOX resistant cancer cells were further investigated.

\subsection{Experimental materials and methods}

\subsubsection{Applied materials and instruments}

Materials and reagents: Absolute ethanol (EtOH, >99.9\%), 3-aminopropyltriethoxysilane (APTS), (4-carboxybutyl) triphenylphosphonium bromide (98\%), Caspase 3 Detection Kit

(Colorimetric, Abcam), Caspase 9 Detection Kit (Colorimetric, Abcam), Multi-drug Resistance Assay Kit (Cayman Chemical), 1-ethyl-3-(3-dimethylaminopropyl) carbodiimide (EDC), cetyltrimethylammonoium bromide (CTAB, 90\%), 4',6-diamidino-2-phenylindole (DAPI), Mitochondrial oxidative phosphorylation inhibitor 99\% (FCCP, Abcam), (3-(4,5dimethylthiazol-2-yl)-2,5-diphenyltetrazolium bromide (MTT), doxorubicin (DOX), dulbecco's modified eagle's medium (DMEM), fluorescein isothiocyanate (FITC), fetal bovine serum (FBS), hydrochloride ( $\mathrm{HCl}, 37 \%)$, N-hydroxysulfosuccinimide (NHS), intracellular ATP determination kit (Invitrogen), Lyso-Tracker Red (Invitrogen), methanol (MeOH, 99.5\%), mitochondria isolation kit for cultured cells (Thermo Scientific), MitoTracker Deep Red (Invitrogen), phosphate buffered saline (PBS), sodium hydroxide (NaOH), and tetraethylorthosilicate (TEOS, 99\%) were purchased commercially.Nanopure water (18.2 $\mathrm{M} \Omega$, Millipore Co., USA) was used in all experiments and buffer preparations.

Instruments:Same as in Chapter 3.

\subsubsection{Synthesis of MSNPs}

The synthesis methodology applied is similar to the procedures in Chapter 3 . 


\subsubsection{DOX loading and releasing}

Firstly, DOX solution with concentration of $1 \mathrm{mg} / \mathrm{mL}$ was prepared as DOX stock solution. The DOX loading process was conducted by suspending $1.0 \mathrm{mg}$ MSNPs in $1.0 \mathrm{~mL}$ DOX stock solution. Then the MSNPs contained DOX stock solution was placed at dark under stirring for $24 \mathrm{~h}$. After that, MSNPs were loaded with DOX molecules due to the diffusion process driven by concentration difference of DOX. The DOX loaded MSNPs were then collected by centrifugation at $10000 \mathrm{rpm}$ for $5 \mathrm{~min}$. Then, the obtained DOX loaded MSNPs were further suspended in DI water and re-centrifuged at $10000 \mathrm{rpm}$ for $5 \mathrm{~min}$. The resuspending process was repeated for three time in order to eliminate the DOX molecules on the surface of MSNPs. The DOX loading capacity of MSNPs was calculated based on the difference of UV-vis intensities at $485 \mathrm{~nm}$ between DOX solution after MSNPs loading and the original DOX stock solution. The DOX loading process was repeated for three time.

As for the in vitroDOX releasing of DOX loaded MSNPs, $500 \mathrm{mg}$ of DOX loaded MSNPs were weighted and suspended in $2.0 \mathrm{~mL}$ PBS solution. After that, the nanoparticles suspended PBS solution was placed under stirring for different periods of time for $1 \mathrm{~h}, 2 \mathrm{~h}, 4$

h, 8 h, 12 h, 24 h, 48 h, 36 h and 72 h. At each time point, the MSNPs contained PBS solution was centrifuged and the supernatant was collected. Then the UV-vis intensities at $485 \mathrm{~nm}$ of each collected supernatant were obtained. The DOX concentrations of each supernatant were calculated based on the obtained UV-vis intensities. Finally, the DOX leasing test was repeated for three times.

\subsubsection{Cell culture}

A2780 cancerous cells (human ovarian cancer cell line) were cultured in DMEM containing 1\% penicillin/streptomycin (Invitrogen) and $10 \%$ FBS (Invitrogen). DOX resistant A2780 (ADR-A2780) cells were cultured in complete DMEM plus DOX $(1 \mu \mathrm{M})$. The cells were 
cultured in incubator which has condition of maintained humidified atmosphere containing $5 \%$ $\mathrm{CO}_{2}$ under $37^{\circ} \mathrm{C}$.

\subsubsection{MTT cytotoxicity assay}

A2780 and ADR-A2780 cells were cultured in a good condition at first. The detailed detection process can be found in Chapter 2 .

\subsubsection{Confocal laser scanning microscopy study}

ADR-A2780 cells were cultured on cover slides in at $1 \times 10^{5}$ cells per well for $24 \mathrm{~h}$. After that, these cells were treated with MSNPs-NH 2 -FITC, MSNPs-PPh 3 -FITC for $24 \mathrm{~h}$, respectively. The mitochondria staining was carried out based on the protocol provided by Invitrogen. Generally, staining solution was prepared with a proper concentration $(50 \mathrm{nM})$ and placed in the dark. Then, the old culturing solution was replaced with a fresh one having mitochondria staining solution $(50 \mathrm{nM})$, and cells were cultured in the incubator for additional 45 min. Following the washing process, stained cells were treated by formaldehyde $(4.0 \%)$. The cells were washed with phosphate-buffered saline (PBS) before the observation by a confocal microscopy.The fluorescence intensity of FITC and Mito-Tracker DeepRed was collected at $\lambda=(520 \pm 10)$ and $(620 \pm 10) \mathrm{nm}$ channels under excitations at $\lambda=488$ and 594 $\mathrm{nm}$, respectively. All the images were obtained sequentially.

\subsubsection{Flow cytometry}

Briefly, A2780 and ADR-A2780 cells seeded in a 6-well plate at cell density of $1 \times 10^{5}$ cells per well for $24 \mathrm{~h}$. The detailed process can be found in Chapter 3 . 


\subsubsection{Mitochondria isolation}

Mitochondria isolation was conducted in A2780 and ADR-A2780 cells. The isolation process was carried out based on the mitochondria isolation kit. The detailed detection process can be found in Chapter 3 .

\subsubsection{Intracellular ATP level detection}

The ATP level detection in A2780 and ARD-A2780 cells was carried out using ATP detection kit, and the detection process was conducted following the protocol provided. Generally, cells were cultured in a 6 -well plate at $1 \times 10^{5}$ cells per well for $24 \mathrm{~h}$. The detaileddetection process can be found in Chapter 3.

\subsubsection{P-gp activity detection}

The activity detection of P-gpwas carried out in A2780 and ADR-A2780 cells. The procedures were followed by multi-drug resistance assay kit provided by Cayman chemical. Generally, the Calcein AM staining solution was prepared at first by adding $2 \mu \mathrm{L}$ provided Calcein AM solution to $10 \mathrm{~mL}$ cell culture medium. Differentcells were cultured in a 96-well plate in a cell density of $5 \times 10^{4}$ cells/well in $100 \mu \mathrm{L}$ of culture medium. Then glow the seeded cells overnight. After that, the old culturing medium was replaced with $100 \mu \mathrm{L}$ fresh medium containing testing MSNPs. For the positive control inhibitor (Cyclosporin A), dilute 1:10000 in culture medium and culture with cells for $30 \mathrm{~min}$. At the end of the treatment, add $100 \mu \mathrm{L}$ prepared Calcein AM staining solution to each well and incubate cells for another $30 \mathrm{~min}$ at culturing condition. Then, the staining solution was removed and $200 \mu \mathrm{L}$ ice cold medium was added into each well. After that, the fluorescent intensities of Calcein AM of sample wells were immediately detected by microplate reader. 


\subsubsection{Mitochondria membrane potential detection}

The mitochondria membrane potential of A2780 and ADR-A2780 cells were detected by applying TMRE mitochondrial membrane potential assay kit. The detection process was carried out following procedures recommended by Abcam. Basically, different cells seeded in a 6 -well plate at $1 \times 10^{5}$ cells per well were incubated for $24 \mathrm{~h}$, respectively. The media were replaced by fresh ones having different MSNP samples. The medium was replaced again with fresh one having TMRE $(200 \mathrm{nM})$ after further $24 \mathrm{~h}$ incubation. Thefluorescence of TMRE excited at $549 \mathrm{~nm}$ was measured. In addition, the mitochondria membrane potential was also detected by usingflow cytometrymethod. Generally, different cells were cultured in a 6 -well plate at $1 \times 10^{5}$ per well for $24 \mathrm{~h}$. Then, old media were replaced by fresh ones having MSNP samples. After further incubation for $24 \mathrm{~h}$, the media were again replaced by fresh media containing TMRE $(200 \mathrm{nM})$. Cells were treated with trypsin and collected by centrifugation. After washing with PBS for two times and harvesting again in $2.0 \mathrm{~mL}$ tube, cells were ready for flow cytometry study. Mitochondrial oxidative phosphorylation inhibitor (FCCP) was set as a positive control.

\subsubsection{Apoptosis study}

The activities of caspase 9 and caspase 3 in A2780 and ADR-A2780 cells were detected by using Caspase 9 Assay Kit (Colorimetric, ab65608) and Caspase 3 Assay Kit (Colorimetric, ab39401) provided by Abcam, respectively. The detection procedures were carried out following the protocol from Abcam. Briefly, different cells in a 6 -well plate at $1 \times 10^{5}$ cells per well were cultured for a period of $24 \mathrm{~h}$. The medium was replaced by new one having MSNP testing samples. Cells were treated by trypsin and pelleted in $2.0 \mathrm{~mL}$ tube. Then, pelleted cells were re-suspended in cold cell lysis buffer $(50 \mu \mathrm{L})$. The cells lysis solution was centrifuged at $10000 \times$ gfor $1 \mathrm{~min}$, and the supernatant was moved to a fresh tube for the assay 
detection. The caspase reaction buffer was prepared according to the provided protocol. The supernatant was added into $96-$-well plate by $50 \mu \mathrm{L}$ per well. Then, reaction buffer $(50 \mu \mathrm{L})$ along with the substrate $(5 \mu \mathrm{L}, 4 \mathrm{mM})$ was added into the tested well. The well was gently mixed, and incubated at $37^{\circ} \mathrm{C}$ for $1.5 \mathrm{~h}$. Finally, the optical intensity $(405 \mathrm{~nm})$ of each tested well was measured by microplate reader. FCCP was applied as positive control group.

\subsection{Result and discussion}

\subsubsection{Fabrication and characterization of MSNPs}

The fabrication process of applied MSNPs was same as mentioned in Chapter 3. Briefly, the average diameter of obtained MSNPs was $65 \mathrm{~nm}$ and the well-ordered structure and large surface area of MSNPs were characterized by methods of powder X-ray diffraction and BET surface area detection. The successful conjugation of TPP on the surface of MSNPs was demonstrated by FT-IR and Zeta potential detections. Please refer to Chapter 3 for more details.

\subsubsection{DOX loading and releasing}

The DOX loading process was the diffusion mechanism of DOX molecules from stock loading solution to the pores of MSNPs. The driving force is the concentration difference of

DOX between loading solution and meso-pores of MSNPs. The obtained DOX loading capacity of MSNPs is $3.35 \mathrm{wt} \%$. The DOX releasing profile of DOX loaded MSNPs was also detected by measuring the released DOX at a series of time intervals, indicating that DOX molecules can be released form MSNPs in a steady and controlled way. The release profile is similar to Figure 5 in Chapter 1.

\subsubsection{Intracellular uptake of free DOX and MSNPs}



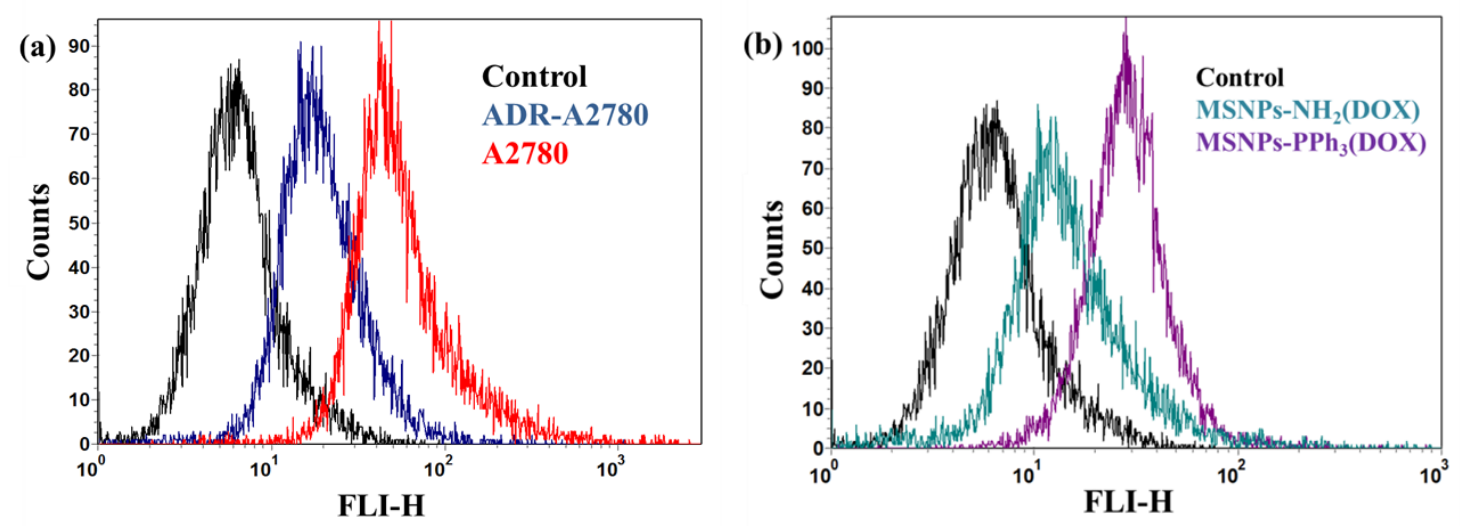

Figure 1. (a) A2780 and ADR-A2780 cells were tested after being treated with free DOX (4 $\mu \mathrm{g} / \mathrm{mL}$ ) (b) ADR-A2780 cells were detected after being treated with MSNPs-NH $\mathrm{N}_{2}(\mathrm{DOX})$ and MSNPs-PPh ${ }_{3}(\mathrm{DOX})(50 \mu \mathrm{g} / \mathrm{mL})$. FL1-H: Fluorescence of DOX.

Since ADR-A2780 cancer cells are resistant to DOX drug molecules, we further investigated the uptake of free DOX molecules both in normal A2780 and ADR-A2780 cells by using flow cytometry (Figure 1). The uptake of DOX loaded MSNPs in ADR-A2780 cells was detected as well by flow cytometry. In the obtained result of flow cytometry detection, it can be observed that free DOX molecules were able to be uptaken effectively by both normal A2780 and ARD-A2780 cells. Whereas in ADR-A2780 cells, lower fluorescence intensity of DOX was observed than that in normal A2780 cells. This observation indicated that the intracellular concentration of free DOX in ADR-A2780 cells was lower than that in normal A2780 cell. The DOX resistant property of ADR-A2780 cells was proved by such observed phenomenon. For the study of MSNPs internalization in ADR-A2780 cells, the obtained result showed that both MSNPs- $\mathrm{NH}_{2}$-DOX and $\mathrm{MSNPs}-\mathrm{PPh}_{3}$-DOX would be effectively internalized into ADR-A2780 cells. However, similarly, the DOX fluorescence intensity detected in MSNPs-NH 2 -DOX group was higher than that detected in $\mathrm{MSNPs}_{2} \mathrm{PPh}_{3}$-DOX groups, indicating that the intracellular concentration of DOX was lower in $\mathrm{MSNPs}-\mathrm{PPh}_{3}$ DOX treated ARD-A2780 cells. Such observation can also be explained by the DOX resistant property of ADR-A2780 cells which can lead the enhanced efflux of DOX molecules and further lowered intracellular DOX concentration. 


\subsubsection{Mitochondria targeting}

In order to investigate the mitochondria targeting property of synthesized MSNPs, confocal laser microscopy method was applied. The mitochondria targeting property was achieved by TPP conjugation on the surface of MSNPs. Therefore, MSNPs-NH $\mathrm{N}_{2}-\mathrm{FITC}$ and $\mathrm{MSNPs}-\mathrm{PPh}_{3}-$ FITC were investigated in A2780 and ADR-A2780 cells. Due to the FITC functionality, MSNPs could be visualized with green color. The intracellular mitochondria were stained with red color (Figure 2).

In the obtained results, clear spots with green color were successfully observed, meaning that fabricated MSNPs could be effectively internalized by ADR-A2780 cells. Besides, the obvious red colored spots were also observed as mitochondria which means that the mitochondria of ADR-A2780 were normally active after the treatment of MSNPs because the staining process was based on the activity of mitochondria with normal membrane potential. In the group of testing MSNPs-NH $2-F I T C$, no obvious merged color (yellow) was observed, indicating that no co-localization effect was occurred between stained mitochondria and MSNPs-NH ${ }_{2}$-FITC. In contrast, however, in the case of sampling with MSNPs-PPh ${ }_{3}$-FITC, very clear yellow colored spots were observed in the merge image, demonstrating the existence of co-localization effect between MSNPs-PPh - FITC and mitochondria in ADRA2780 cancer cells. Such observation indicated that after conjugation of TPP on the surface, MSNPs presented effective mitochondria targeting effect facilitated by TPP functionality. It is fully documented that the mitochondria targeting mechanism of TPP is due to its positive molecular charge and high lipophilicity. Since that normally, mitochondria are always highly negative-charged, owing to the electrostatic interaction between TPP and mitochondria, TPP functionalized MSNPs will be preferentially accumulated in mitochondria. In addition, the lipophilicity of TPP is beneficial in facilitating the trans-membrane process of MSNPs. As a result, TPP conjugated MSNPs exhibit effective mitochondria targeting property, which is 
indicated by the results of confocal microscopy study. What's more, the co-localization efficient values of all merged images were calculated in Image $J$ software. In figure2a, such values were calculated as 0.249 versus 0.668 and 0.218 versus 0.562 for Pearson's Coefficient and Overlap Coefficient, respectively. Similarly, figure $2 b$, such values were calculated as 0.223 versus 0.725 and 0.271 versus 0.689 , respectively.

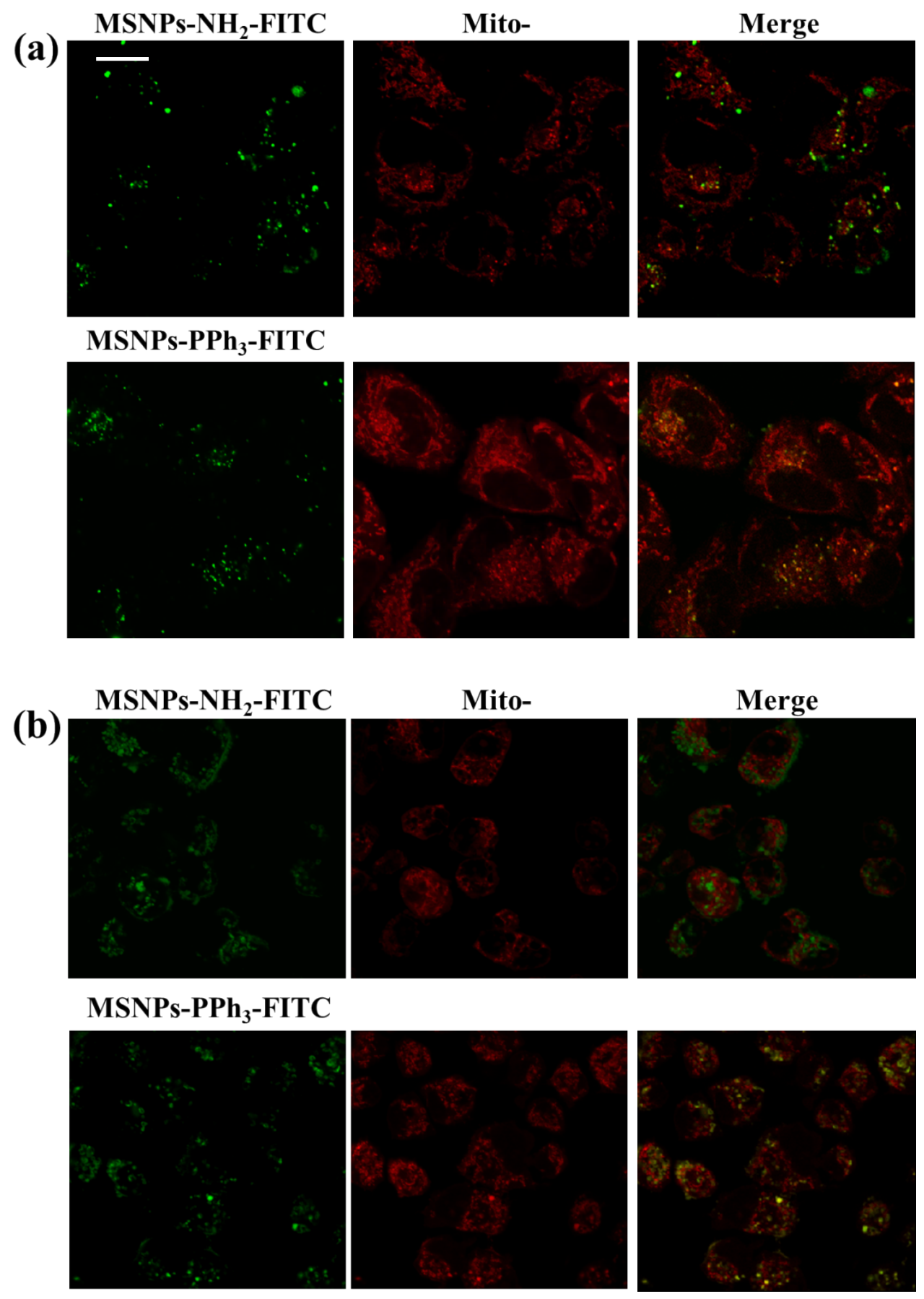


Figure 2. Confocal microscopy images of (a) A2780 and (b) ADR-A2780 cells after being treated with MSNPs-NH ${ }_{2}$-FITC and MSNPs-PPh 3 -FITC $(50 \mu \mathrm{g} / \mathrm{mL})$ for $24 \mathrm{~h}$. Scale bar: 20 $\mu \mathrm{m}$.

\subsubsection{Fluorescence study of isolated mitochondria}

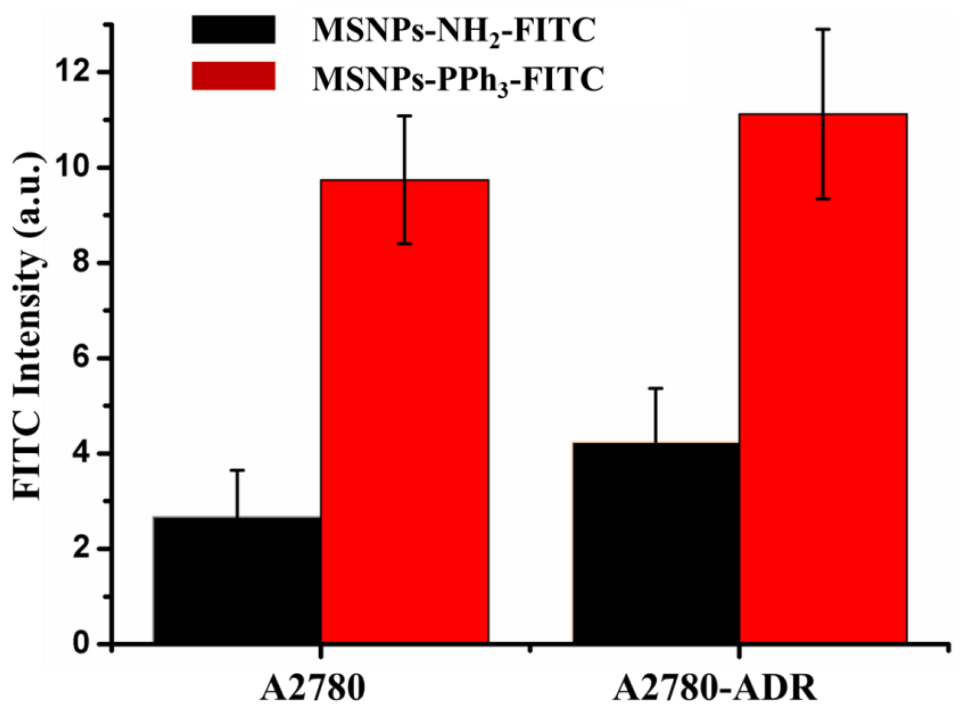

Figure 3.FITC fluorescence intensities in A2780 and ADR-A2780 cells after being treated with MSNPs-NH ${ }_{2}$-FITC and MSNPs-PPh ${ }_{3}$-FITC $(50 \mu \mathrm{g} / \mathrm{mL})$.

In order to further demonstrate the targeting property of $\mathrm{MSNPs}-\mathrm{PPh}_{3}-\mathrm{FITC}$, the FITC fluorescence intensity detection was conducted in normal A2780 and ADR-A2780 cells after being treated with MSNPs (Figure 3), similar with method applied in Chapter 3. From the results, it can be seen that in the group of testing MSNPs- $\mathrm{NH}_{2}$-FITC, there was no significant FITC fluorescence intensities detected in mitochondria isolated from both normal A2780 cells and ADR-A2780 cells. Considering the fact that the only trace of FITC fluorescence signal is come from MSNPs under such circumstance, there was no significant amount of MSNPs existing in mitochondria isolated form A2780 and ADR-A2780 cells. However, when it comes to the case of testing MSNPs- $\mathrm{PPh}_{3}$-FITC, in the isolated mitochondria from both normal A2780 and ADR-A2780 cells, significant FITC fluorescence intensities were detected, indicating the existence of MSNPs-PPh ${ }_{3}$-FITC in mitochondria. Such observation directly demonstrated that TPP conjugated MSNPs were capable of being selectively 
accumulated in mitochondria, further proving the effectiveness of $\mathrm{MSNPs}-\mathrm{PPh}_{3}$ in targeting mitochondria in A2780 and ADR-A2780 cells together with the positive results obtained from confocal microscopy study.

\subsubsection{Cytotoxicity and mitochondrial dysfunctions}

The cytotoxicity of MSNPs-PPh $-\mathrm{DOX}$ and $\mathrm{MSNPs}-\mathrm{NH}_{2}-\mathrm{DOX}$ can be directly investigated by applying MTT assay. The MTT assay detection is based on the mechanism that living cells can generate the chemical substrate of MTT through various metabolic chemical reactions, resulting in the formation of purple colored compound when dissolved in DMSO solution. As a result, the darker of resulted purple color, the more active of tested cells. The resulted cytotoxicity is represented by survival ratio.
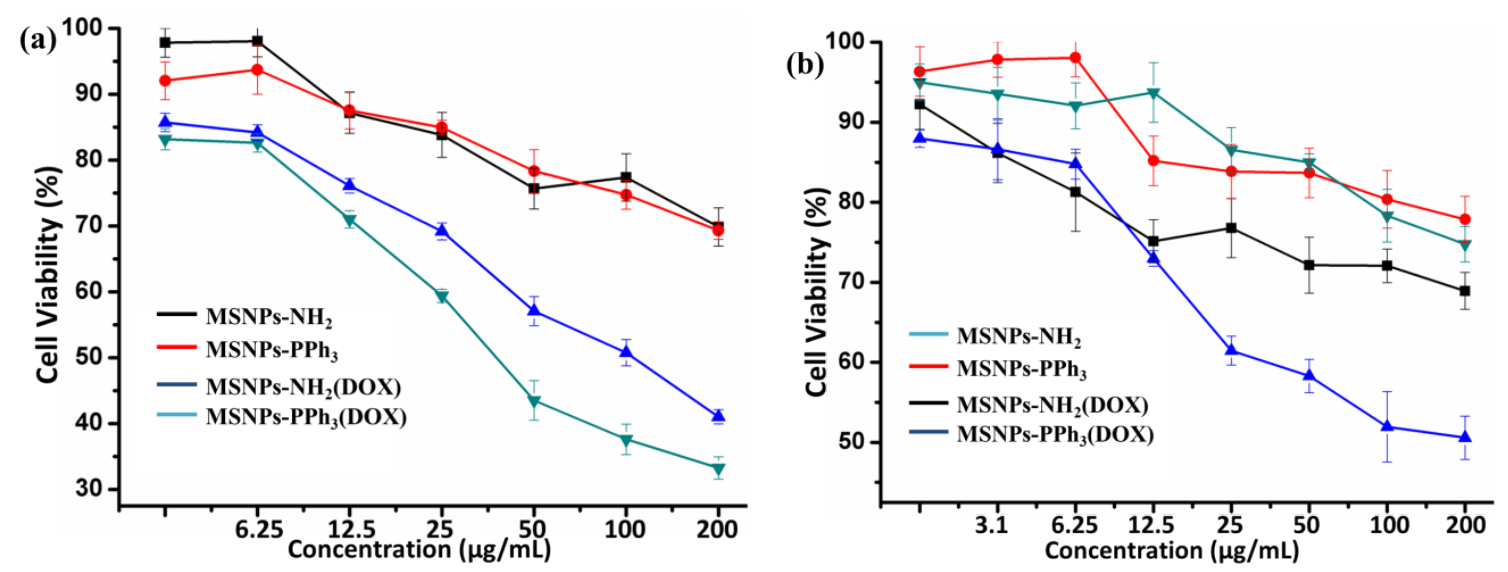

Figure 4.Cytotoxicity of (a) A2780 and (b) ADR-A2780 cells after being treated with different MSNPs for $24 \mathrm{~h}$.

In the obtained results (Figure 4), it could be observed that for testing MSNPs samples in normal A2780 cells, MSNPs without DOX loading presented high cell survival ratio (more than $70 \%$ ) even at higher MSNPs concentrations up to $200 \mu \mathrm{g} / \mathrm{mL}$, meaning that pure MSNPs (MSNPs-NH $\mathrm{N}_{2}$ and MSNPs-PPh ${ }_{3}$ ) did not present cytotoxicity toward normal A2780 cells. When treating normal A2780 cells with DOX loaded MSNPs (MSNPs-PPh - DOX and MSNPs- $\mathrm{NH}_{2}$-DOX), the cell survival rate decreased along with the increase of MSNPs 
concentration, indicating that both MSNPs-PPh ${ }_{3}-\mathrm{DOX}$ and MSNPs-NH $\mathrm{N}_{2}-\mathrm{DOX}$ showed good cytotoxicity against normal A2780 cells. At the meantime, in the test of MSNPs samples in ADR-A2780 cells, similarly, MSNPs without DOX loading still presented low cytotoxicity. However, when ADR-A2780 cells were treated with DOX loaded MSNPs (MSNPs-PPh ${ }^{-}$ DOX and MSNPs- $\mathrm{NH}_{2}$-DOX), the cell survival rate related to $\mathrm{MSNPs}-\mathrm{NH}_{2}-\mathrm{DOX}$ was observed to be more than $70 \%$ even at higher concentrations which means that $\mathrm{MSNPs}^{-\mathrm{NH}_{2}-}$ DOX did not show effective cytotoxicity in ADR-A2780 cells. Nevertheless, around half of ADR-A2780 cells treated with MSNPs-PPh $-\mathrm{DOX}$ were killed at concentration of 100 and $200 \mu \mathrm{g} / \mathrm{mL}$, indicating that $\mathrm{MSNPs}-\mathrm{PPh}_{3}-\mathrm{DOX}$ presented a relatively much better killing efficiency in ADR-A2780 cells. Accordingly, MSNPs-PPh ${ }_{3}$-DOX showed good effectiveness in overcoming DOX resistance. Such effectiveness is achieved by the effective delivery of DOX into mitochondria. In the case of applying MSNPs- $\mathrm{NH}_{2}-\mathrm{DOX}$, the DOX molecules are mostly delivered into cytoplasm by MSNPs and the acting way of DOX molecules in cytoplasm is to interact with DNA molecules in the nucleus. However, in the case of using MSNPs- $\mathrm{PPh}_{3}$-DOX, the DOX molecules are effectively delivered into mitochondria, leading to the interaction between delivered DOX molecules and mtDNA molecules (DNA inside mitochondria) and further malfunctions of mitochondria. In ADR-A2780 cells, due to drug resistant property, DOX molecules in cytoplasm delivered by MSNPs- $\mathrm{NH}_{2}$ will be transported to ex-cellular space, resulting in the decreased intracellular concentration of DOX. Thus, MSNPs- $\mathrm{NH}_{2}-\mathrm{DOX}$ are effective in overcoming DOX resistance in ADR-A2780 cells.

The malfunctions of mitochondria caused by MSNPs-PPh $-\mathrm{DOX}$ were further investigatedin both normal A2780 and ADR-A2780 cells. Here we detected the alterations in intracellular ATP production level as well as mitochondrial membrane potential. In the result of mitochondrial membrane potential study (Figure 5), it is shown that MSNPs without DOX loading (MSNPs- $\mathrm{NH}_{2}$ and $\mathrm{MSNPs}-\mathrm{PPh}_{3}$ ) did not have obvious effect in reducing the 
mitochondrial membrane potential for both normal A2780 and ADR-A2780 cells. Similarly, in testing with MSNPs-NH ${ }_{2}$-DOX in ADR-A2780 cells, no obvious decease of mitochondrial membrane potential was observed, which means that DOX molecules delivered by MSNPs without TPP functionality could not result membrane potential lose in mitochondria. But in normal A2780 cells with MSNPs- $\mathrm{NH}_{2}$-DOX, a clear decrease of mitochondrial membrane potential was observed. In the case of sampling with $\mathrm{MSNPs}-\mathrm{PPh}_{3}$-DOX, however, both normal A2780 and ADR-A2780 showed obvious decreased mitochondrial membrane potential, indicating that DOX molecule delivered by MSNPs with TPP functionality presented good ability in inducing membrane potential depletion in mitochondria. The similar result was obtained by applying flow cytometry study. FCCP was introduced as positive control.
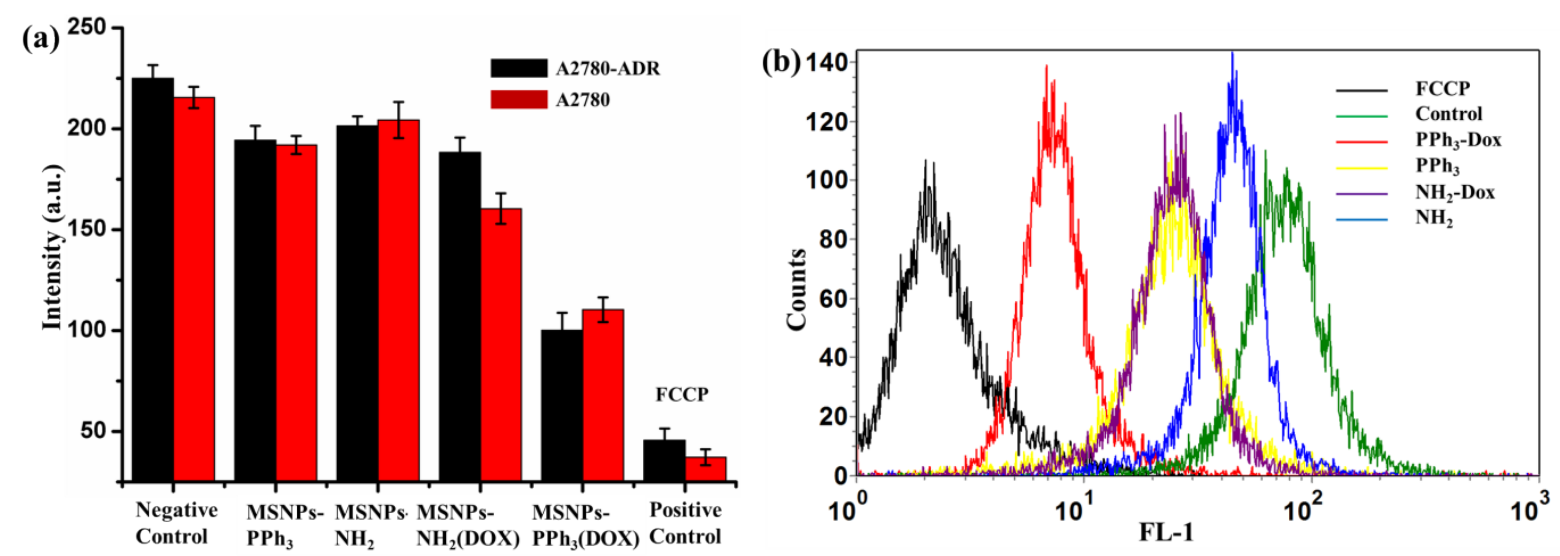

Figure 5. (a) TMRE fluorescence intensities of A2780 and ADR-A2780 cells treated with different MSNPs samples $(50 \mu \mathrm{g} / \mathrm{mL})$. (b) Flow cytometry of ADR-A2780 cells treated with different MSNPs samples. FL-1: TMRE fluorescence.

In the study of intracellular ATP production level in ADR-A2780 cells, the results showed that MSNPs without DOX loading presented no clear effect on ATP production level corresponding with the non-cytotoxicity result obtained in cytotoxicity study (Figure 6). A little decrease of ATP level caused by MSNPs- $\mathrm{PPh}_{3}$ was observed which might be due to the existence of MSNPs- $\mathrm{PPh}_{3}$ inside mitochondria. In testing with MSNPs- $\mathrm{NH}_{2}-\mathrm{DOX}$, there was no significant observed decrease of ATP production level in ADR-A2780 cells. However, in 
the case of applying MSNPs- $\mathrm{PPh}_{3}$-DOX, the intracellular ATP production level was dramatically decreased comparing with that in negative control group. Such observation clearly indicated the active interaction between delivered DOX molecules and mtDNA molecules was achieved, leading to the alteration in ATP production. Above all, it can be concluded that MSNPs-PPh $-\mathrm{DOX}$ were able to effectively induce cell death in ADR-A2780 cells by affecting normal functions of mitochondria such as membrane potential and intracellular ATP production, which demonstrated, more importantly, the effectiveness in overcoming DOX resistance in ADR-A2780 cells.
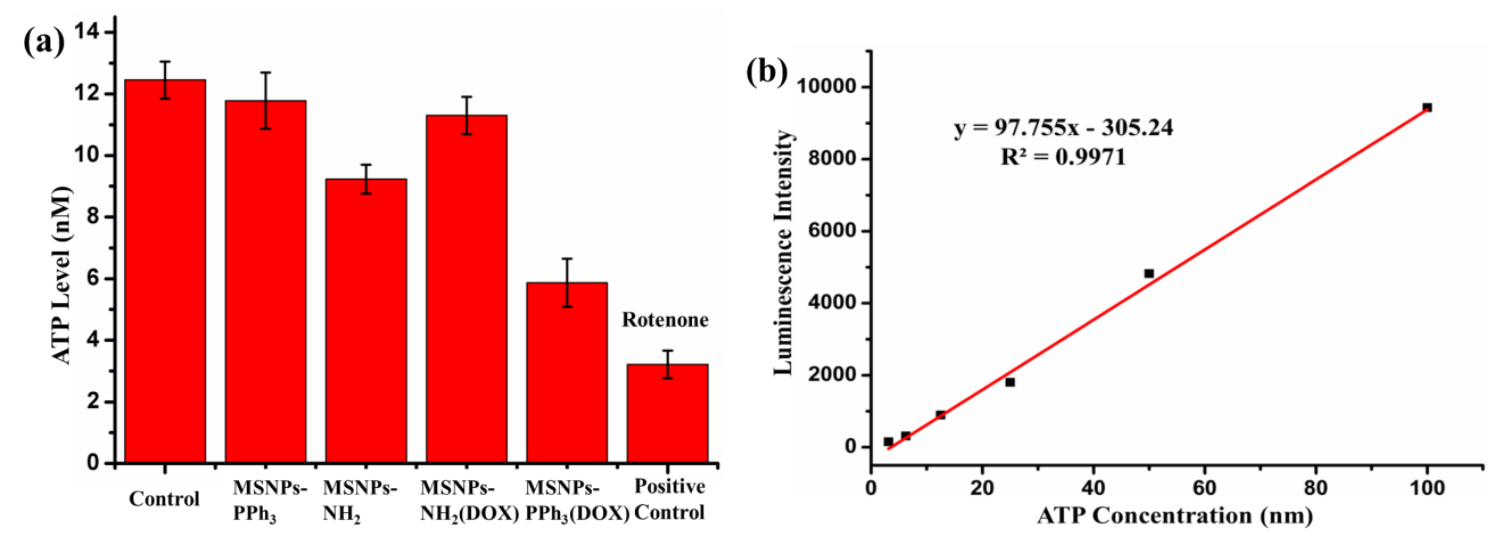

Figure 6.(a) Intracellular ATP production level in ADR-A2780 cells after being treated with different MSNPs $(50 \mu \mathrm{g} / \mathrm{mL})$.(b) Standard curve of intracellular ATP concentration.

\subsubsection{Detection of P-gp activity}

As mentioned that P-gp activity is the key aspect in developing and maintaining drug resistance. Active P-gp can result in the effective efflux of anticancer drug molecules to excellular space, reducing the intracellular concentration of such anticancer drug. Therefore, it is quite necessary and important to determine the activity of P-gp and its possible change when introducing our designed MSNPs. The detection of the activity of P-gp follows protocol provided in the multi-drug resistance assay kit. The utilization of such detection kit is based on the mechanism that active P-gp will be responsible for the efflux of fluorescent Calcein AM molecules out of cellular membrane. By measuring the fluorescence intensity of 
remained intracellular Calcein AM, the activity of P-gpcan be detected. Here the P-gpactivity is marked as Calcein AM retention percentage. The higher Calcein AM retention percentage, the lower activity of $\mathrm{P}$-gp in that most Calcein AM molecules are still remaining intracellularly in such case. Cycloporin A is set as positive control group, which can inhibit the function of P-gp.
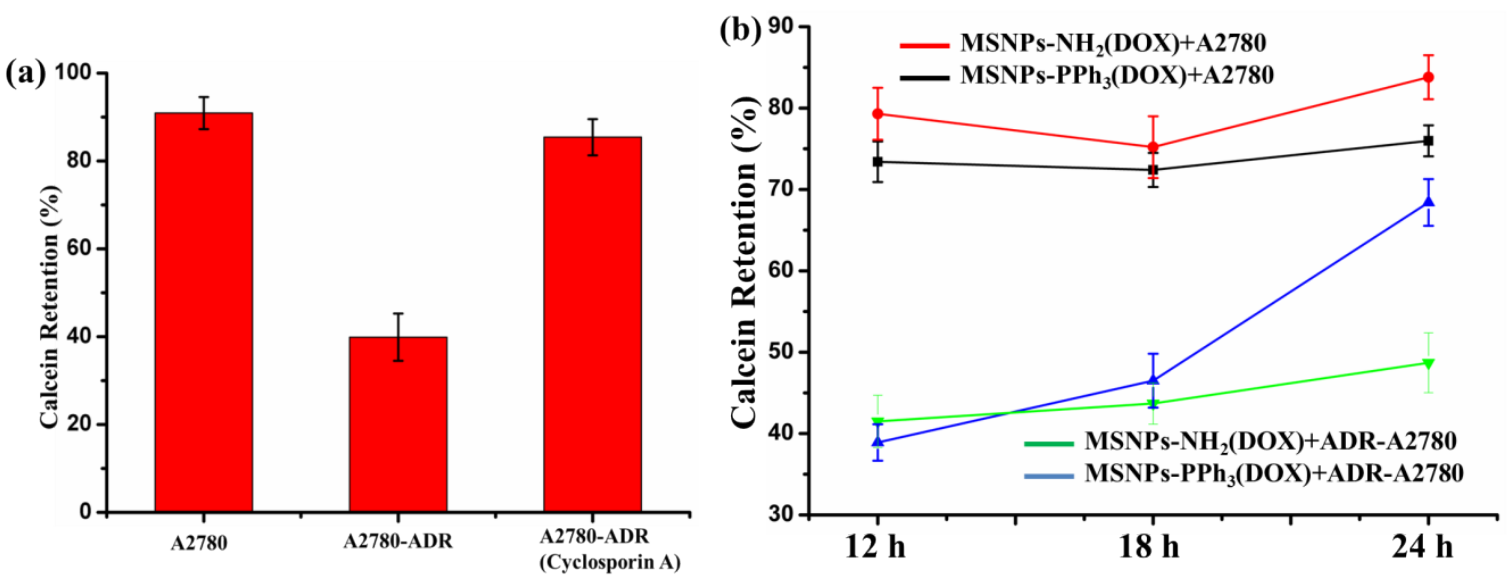

Figure 7.(a) P-gp activities in A2780 and ADR-A2780 cells. (b) Time-dependent change of P-gp activity of A2780 and ADR-A2780 cells treated with different DOX loaded MSNPs (50 $\mu \mathrm{g} / \mathrm{mL})$.

In the obtained result (Figure 7), highCalcein AM retention percentage was observed when testing MSNPs- $\mathrm{NH}_{2}$-DOX, presenting that the activity of P-gp was not affected by DOX molecules delivered by MSNPs- $\mathrm{NH}_{2}$. However, in the case of detecting MSNPs- $\mathrm{PPh}_{3}$-DOX, the Calcein AM retention percentage was decreased obviously, indicating that Calcein AM molecules could be effectively pumped out of cell membrane. Furthermore, such indication represented the high activity of P-gp in this case. The observation presented that DOX molecules delivered by MSNPs- $\mathrm{PPh}_{3}$ could effectively lead to the inhibition of P-gp activity. In addition, by considering the fact that activity of P-gpis achieved highly dependent on ATP supply, the P-gp inhibition effect caused by MSNPs-PPh $-\mathrm{DOX}$ was achieved based on the decrease of ATP production. Beyond that, the time dependent change of P-gp activity affected by MSNPs-PPh 3 -DOX was investigatedin both normal A2780 and ADR-A2780 cells as well. The Calcein AM retention percentages of cells treating with $\mathrm{MSNPs}-\mathrm{PPh}_{3}-\mathrm{DOX}$ for 
$12 \mathrm{~h}, 18 \mathrm{~h}$ and $24 \mathrm{~h}$ were detected. The results showed that after $12 \mathrm{~h}$ treatment with MSNPs$\mathrm{PPh}_{3}$-DOX, both $\mathrm{A} 2780$ cells presented high Calcein AM retention percentage and ADRA2780 cells presented relatively low retention percentage, meaning that at that time, P-gp activity was low in A2780 cells and high in ADR-A2780 cells. However, after $18 \mathrm{~h}$ and $24 \mathrm{~h}$ incubation with MSNPs- $\mathrm{PPh}_{3}-\mathrm{DOX}$, the Calcein $\mathrm{AM}$ retention percentages were still high whereas such percentages became lower and lower in ADR-A2780 cells than that in the $12 \mathrm{~h}$ incubation. The observation indicated that the P-gp activity in ADR-A2780 cells was becoming lower with incubation time with MSNPs-PPh $-\mathrm{DOX}$, which was resulted from the decreased ATP production caused by DOX molecules delivered by MSNPs-PPh 3 . Above all, we can conclude that P-gp activity in DOX resistant ADR-A2780 cells would be inhibited by MSNPs-PPh ${ }_{3}$-DOX and such inhibition effect was achieved by decreasing the ATP energy supply for P-gp. The inhibition effect was in a time dependent manner.

\subsubsection{Apoptosis study}

The activation of apoptosis process is closely related to the activation of activities of Caspase 9 and Caspase 3. In order to further investigate the effectiveness of $\mathrm{MSNPs}^{-\mathrm{PPh}_{3}-}$ DOX in inducing cell death, the activities of Caspase 9 and Caspase 3 were investigated in both normal A2780 cells and ADR-A2780 cells. The Caspase 9 and Caspase 3 activities after treating cells with different MSNPs for $12 \mathrm{~h}, 18 \mathrm{~h}$, and $24 \mathrm{~h}$ were measured. FCCP was set as positive control group. For both Caspase 9 and Caspase 3 activity detection, higher activity indicates higher apoptosis activity.

As shown in the obtained results (Figure 8), it can be found that in normal A2780 cells, activities of both Caspase 9 and Caspase 3 was becoming higher along with the incubation time with both MSNPs-PPh $-\mathrm{DOX}$ and MSNPs-NH $\mathrm{N}_{2}$-DOX. Such results showed that both MSNPs- $\mathrm{NH}_{2}$-DOX and MSNPs-PPh ${ }_{3}$-DOX could effectively activate the apoptosis process. 
But, in the case of applying MSNPs- $\mathrm{NH}_{2}-\mathrm{DOX}$ in ADR-A2780 cells, the activities of Caspase 9 and Caspase 3 were relatively low, indicating that MSNPs- $\mathrm{NH}_{2}-\mathrm{DOX}$ were not able to activate the apoptosis process in DOX resistant cells. However, in testing MSNPs$\mathrm{PPh}_{3}$-DOX in ADR-A2780 cells, the activities of Caspase 9 and Caspase 3 were still high. Such observation demonstrated that MSNPs- $\mathrm{PPh}_{3}-\mathrm{DOX}$ were capable of inducing apoptosis in DOX resistant ADR-A2780 cells. The induction of apoptosis process can be attributed to the malfunctions of mitochondria caused by MSNPs-PPh $-\mathrm{DOX}$, which has been discussed before. The positive apoptosis results further indicates the strong ability of $\mathrm{MSNPs}-\mathrm{PPh}_{3}$ DOX in overcoming DOX resistance.
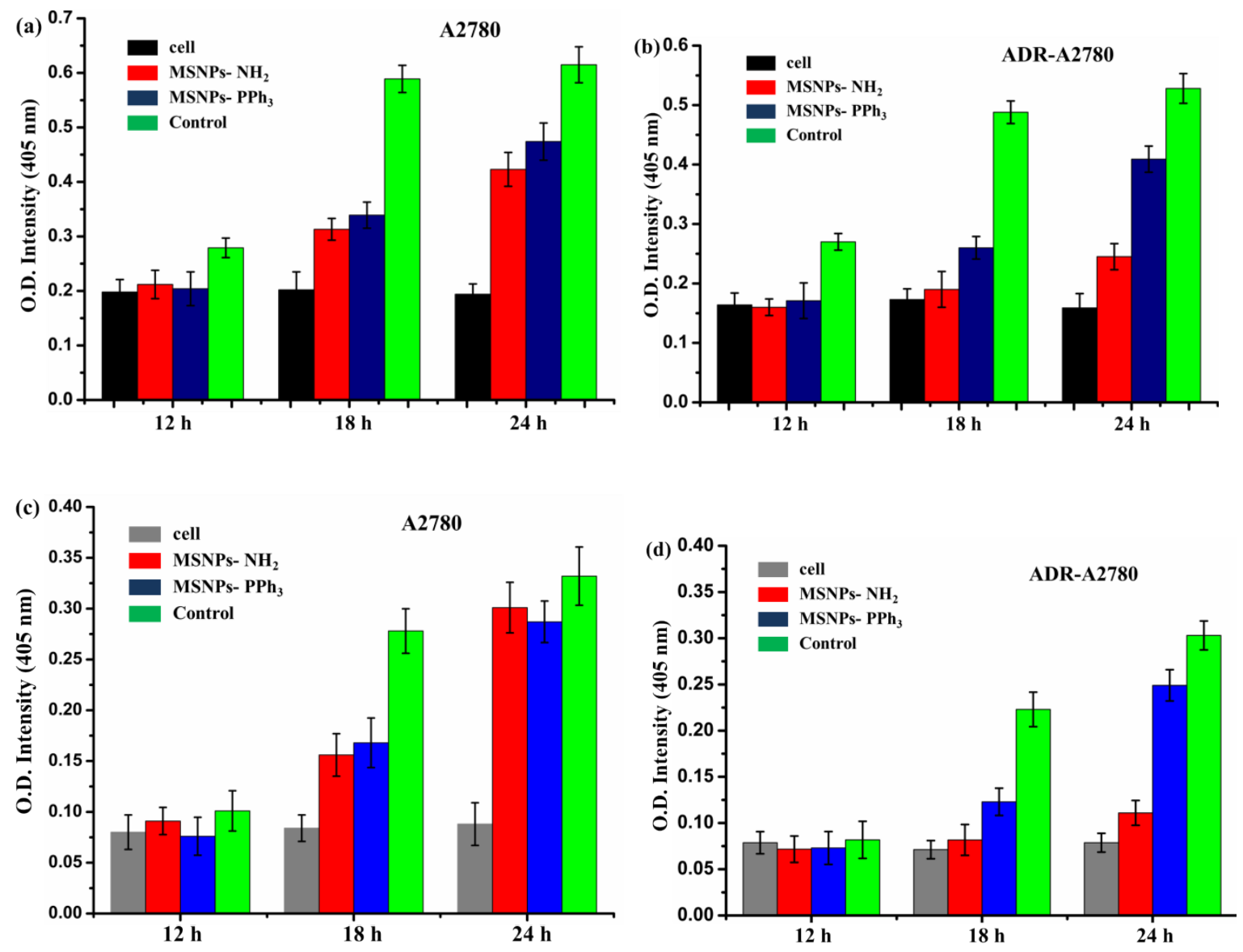

Figure 8.Time-dependent apoptosis study of different MSNPs $(50 \mu \mathrm{g} / \mathrm{mL})$ in A2780 and ADR-A2780 cells. Caspase 9 (a,b), Caspase 3 (c,d). 


\subsection{Conclusion}

In summary, the MSNPs with surface functionality of TPP as effective mitochondria targeting ligand were successfully fabricated. The effective mitochondria targeting property was demonstrated in DOX resistant ADR-A2780 cells together with the fluorescence study of isolated mitochondria. Besides, the fabricated MSNPs were loaded with DOX drug molecules and the effective release of DOX from MSNPs was investigatedin vitro as well. In addition, the anticancer cytotoxicity result showed that $\mathrm{MSNPs}-\mathrm{PPh}_{3}$-DOX could effective inhibit the proliferation of ADR-A2780 cells and the alterations in mitochondrial functions revealed that MSNPs- $\mathrm{PPh}_{3}$-DOX were capable of inhibiting intracellular ATP production and inducing the depletion of mitochondrial membrane potential. Beyond that, the anti-DOX resistance mechanism study indicated that the function of $\mathrm{MSNPs}-\mathrm{PPh}_{3}-\mathrm{DOX}$ was achieved by inhibiting the activity of P-gp. Finally, the apoptosis study demonstrated that $\mathrm{MSNPs}-\mathrm{PPh}_{3}$ DOX could effectively induce apoptosis process by resulted enhanced activities of Caspase 9 and Caspase 3. As a result, the current research suggests MSNPs with mitochondria targeting capability in effective overcoming drug resistance.

\section{References}

1. G. Attardi and G. Schatz, Annu. Rev. Cell. Biol., 1988, 4, 289-333.

2. D. D. Newmeyer and S. Ferguson-Miller, Cell, 2003, 112, 481-490.

3. J. W. O. Ballard and M. C. Whitlock, Mol. Ecol., 2004, 13, 729-744.

4. S. G. E. Andersson, A. Zomorodipour, J. O. Andersson, T. Sicheritz-Ponten, U. C. M. Alsmark, R. M. Podowski, A. K. Naslund, A. S. Eriksson, H. H. Winkler and C. G. Kurland, Nature, 1998, 396, 133-140.

5. D. C. Chan, Cell, 2006, 125, 1241-1252.

6. J. Bereiterhahn and M. Voth, Microsc. Res. Tech., 1994, 27, 198-219.

7. R. S. Balaban, S. Nemoto and T. Finkel, Cell, 2005, 120, 483-495.

8. S. S. Korshunov, V. P. Skulachev and A. A. Starkov, Febs Letters, 1997, 416, 15-18.

9. M. P. Murphy, Biochem. J., 2009, 417, 1-13.

10. S. R. Bacman, S. L. Williams, D. Hernandez and C. T. Moraes, Gene Ther., 2007, 14, 1309-1318.

11. J. P. Abrahams, A. G. W. Leslie, R. Lutter and J. E. Walker, Nature, 1994, 370, 621-628.

12. M. R. Duchen, Journal of Physiology-London, 1999, 516, 1-17.

13. D. W. Hailey, A. S. Rambold, P. Satpute-Krishnan, K. Mitra, R. Sougrat, P. K. Kim and J. Lippincott-Schwartz, Cell, 2010, 141, 656-667.

14. D. G. Nicholls and S. L. Budd, Physiol. Rev., 2000, 80, 315-360. 
15. J. Nunnari and A. Suomalainen, Cell, 2012, 148, 1145-1159.

16. M. S. Suleiman, A. P. Halestrap and E. J. Griffiths, Pharmacol. Ther., 2001, 89, 29-46.

17. A. Atlante, S. Gagliardi, E. Marra and P. Calissano, Neurosci. Lett., 1998, 245, 127-130.

18. E. S. G. Barron, J. A. Muntz and B. Gasvoda, Journal of General Physiology, 1948, 32, 163-178.

19. M. Brunori, A. Giuffre, P. Sarti, G. Stubauer and M. T. Wilson, Cell. Mol. Life Sci., 1999, 56, 549-557.

20. E. Gnaiger, in Hypoxia: Through the Lifecycle, eds. R. C. Roach, P. D. Wagner and P. H. Hackett, 2003, 543, 39-55.

21. E. Gnaiger, R. SteinlechnerMaran, G. Mendez, T. Eberl and R. Margreiter, J. Bioenerg. Biomembr., 1995, 27, 583-596.

22. K. Heerlein, A. Schulze, L. Hotz, P. Bartsch and H. Mairbaurl, Am. J. Respir. Cell Mol. Biol., 2005, 32, 44-51.

23. M. Lehmann, W. Baumann, M. Brischwein, H. J. Gahle, I. Freund, R. Ehret, S. Drechsler, H. Palzer, M. Kleintges, U. Sieben and B. Wolf, Biosensors Bioelectro., 2001, 16, 195-203.

24. D. J. Marcinek, W. A. Ciesielski, K. E. Conley and K. A. Schenkman, Am. J. Physiol.-Heart and Circulatory Physiology, 2003, 285, H1900-H1908.

25. P. Neubauer, H. Y. Lin and B. Mathiszik, Biotechnol. Bioeng., 2003, 83, 53-64.

26. C. J. Songer and J. J. Mintzes, J. Res. Sci. Teaching, 1994, 31, 621-637.

27. Q. H. Tran and G. Unden, Eur. J. Biochem., 1998, 251, 538-543.

28. C. Wanka, J. P. Steinbach and J. Rieger, J. Biol. Chem., 2012, 287, 33436-33446.

29. A. Bentmann, M. Schmidt, S. Reuss, U. Wolfrum, T. Hankeln and T. Burmester, J. Biol. Chem., 2005, 280, 20660-20665.

30. N. S. Chandel, G. R. S. Budinger, S. H. Choe and P. T. Schumacker, J. Biol. Chem., 1997, 272, 18808-18816.

31. M. Wikstrom, Chem. Scripta, 1987, 27B, 53-58.

32. S. L. Wu, M. J. Sampson, W. K. Decker and W. J. Craigen, Biochim. Et Biophysi. Acta-Mol. Cell Res., 1999, 1452, 68-78.

33. G. Hajnoczky, L. D. Robbgaspers, M. B. Seitz and A. P. Thomas, Cell, 1995, 82, 415-424.

34. W. J. Nicklas, I. Vyas and R. E. Heikkila, Life Sci., 1985, 36, 2503-2508.

35. R. Rizzuto, M. Brini, M. Murgia and T. Pozzan, Science, 1993, 262, 744-747.

36. C. Schnaitman and J. W. Greenawalt, J. Cell Biol., 1968, 38, 158-+.

37. D. Decaudin, I. Marzo, C. Brenner and G. Kroemer, Int. J. Oncol., 1998, 12, 141-152.

38. L.-F. Dong, R. Freeman, J. Liu, R. Zobalova, A. Marin-Hernandez, M. Stantic, J. Rohlena, K. Valis, S. Rodriguez-Enriquez and B. Butcher, Clin. Cancer Res., 2009, 15, 1593-1600.

39. H. J. Leenders, A. Kemp, J. F. J. Koninkx and J. Rosing, Exp. Cell Res., 1974, 86, 25-30.

40. D. R. Green and J. C. Reed, Science, 1998, 281, 1309-1312.

41. A. Gross, J. M. McDonnell and S. J. Korsmeyer, Genes Dev., 1999, 13, 1899-1911.

42. J. M. Jurgensmeier, Z. H. Xie, Q. Deveraux, L. Ellerby, D. Bredesen and J. C. Reed, PNAS, 1998, 95, 4997-5002.

43. R. M. Kluck, E. BossyWetzel, D. R. Green and D. D. Newmeyer, Science, 1997, 275, 1132-1136.

44. M. Mihara, S. Erster, A. Zaika, O. Petrenko, T. Chittenden, P. Pancoska and U. M. Moll, Mol. Cell, 2003, 11, 577-590.

45. M. Chen and J. Wang, Apoptosis, 2002, 7, 313-319.

46. P. Costantini, E. Jacotot, D. Decaudin and G. Kroemer, J. Nat. Cancer Inst., 2000, 92, 1042-1053.

47. J. Yang, X. Liu, K. Bhalla, C. N. Kim, A. M. Ibrado, J. Cai, T.-I. Peng, D. P. Jones and X. Wang, Science, 1997, 275, 1129-1132.

48. Y. Zhao, R. Li, W. Xia, J. Neuzil, Y. Lu, H. Zhang, X. Zhao, X. Zhang, C. Sun and K. Wu, Cancer lett., 2010, 288, 42-49.

49. M. J. Arends, R. G. Morris and A. H. Wyllie, Am. J, Pathol., 1990, 136, 593-608.

50. A. R. Clarke, C. A. Purdie, D. J. Harrison, R. G. Morris, C. C. Bird, M. L. Hooper and A. H. Wyllie, Nature, 1993, 362, 849-852.

51. G. M. Cohen, Biochem. J., 1997, 326, 1-16.

52. S. Elmore, Toxicol. Pathol., 2007, 35, 495-516.

53. M. O. Hengartner, Nature, 2000, 407, 770-776.

54. J. F. R. Kerr, C. M. Winterford and B. V. Harmon, Cancer, 1994, 73, 2013-2026.

55. P. E. Czabotar, G. Lessene, A. Strasser and J. M. Adams, Nat. Rev. Mol. Cell. Biol., 2014, 15, 4963. 
56. W. C. Earnshaw, L. M. Martins and S. H. Kaufmann, Annu. Rev. Biochem., 1999, 68, 383-424.

57. S. Nagata, Cell, 1997, 88, 355-365.

58. T. Nakagawa, H. Zhu, N. Morishima, E. Li, J. Xu, B. A. Yankner and J. Y. Yuan, Nature, 2000, 403, 98-103.

59. X. Saelens, N. Festjens, L. Vande Walle, M. van Gurp, G. van Loo and P. Vandenabeele, Oncogene, 2004, 23, 2861-2874.

60. A. L. Horwich, F. Kalousek, W. A. Fenton, R. A. Pollock and L. E. Rosenberg, Cell, 1986, 44, 451-459.

61. G. F. Kelso, C. M. Porteous, C. V. Coulter, G. Hughes, W. K. Porteous, E. C. Ledgerwood, R. A. J. Smith and M. P. Murphy, J. Biol. Chem., 2001, 276, 4588-4596.

62. I. M. Moller, Annu. Rev. Plant Physiol. Plant Mol. Biol., 2001, 52, 561-591.

63. T. L. Vanden Hoek, L. B. Becker, Z. H. Shao, C. Q. Li and P. T. Schumacker, J. Biol. Chem., 1998, 273, 18092-18098.

64. D. Narendra, A. Tanaka, D.-F. Suen and R. J. Youle, J. Cell Biol., 2008, 183, 795-803.

65. X. D. Wang, Genes Dev., 2001, 15, 2922-2933.

66. D. Xia, C. A. Yu, H. Kim, J. Z. Xian, A. M. Kachurin, L. Zhang, L. Yu and J. Deisenhofer, Science, 1997, 277, 60-66.

67. D. Hanahan and R. A. Weinberg, cell, 2011, 144, 646-674.

68. I. A. Aligianis, C. A. Johnson, P. Gissen, D. R. Chen, D. Hampshire, K. Hoffmann, E. N. Maina, N. V. Morgan, L. Tee, J. Morton, J. R. Ainsworth, D. Horn, E. Rosser, T. R. P. Cole, I. StolteDijkstra, K. Fieggen, J. Clayton-Smith, A. Megarbane, J. P. Shield, R. Newbury-Ecob, W. B. Dobyns, J. M. Graham, K. W. Kjaer, M. Warburg, J. Bond, R. C. Trembath, L. W. Harris, Y. Takai, S. Mundlos, D. Tannahill, C. G. Woods and E. R. Maher, Nat. Genet., 2005, 37, 221-223.

69. R. Diaz-Ruiz, M. Rigoulet and A. Devin, Biochimica Et Biophysica Acta-Bioenergetics, 2011, 1807, 568-576.

70. B. Faubert, G. Boily, S. Izreig, T. Griss, B. Samborska, Z. F. Dong, F. Dupuy, C. Chambers, B. J. Fuerth, B. Viollet, O. A. Mamer, D. Avizonis, R. J. DeBerardinis, P. M. Siegel and R. G. Jones, Cell Metab., 2013, 17, 113-124.

71. J. Favier, J. J. Briere, N. Burnichon, J. Riviere, L. Vescovo, P. Benit, I. Giscos-Douriez, A. De Reynies, J. Bertherat, C. Badoual, F. Tissier, L. Amar, R. Libe, P. F. Plouin, X. Jeunemaitre, P. Rustin and A. P. Gimenez-Roqueplo, Plos One, 2009, 4.

72. M. G. Vander Heiden, L. C. Cantley and C. B. Thompson, Science, 2009, 324, 1029-1033.

73. $\quad$ K. Garber, J. Nat. Cancer Inst., 2004, 96, 1805-1806.

74. M. G. V. Heiden, L. C. Cantley and C. B. Thompson, Science, 2009, 324, 1029-1033.

75. P. P. Hsu and D. M. Sabatini, Cell, 2008, 134, 703-707.

76. A. Isidoro, E. Casado, A. Redondo, P. Acebo, E. Espinosa, A. M. Alonso, P. Cejas, D. Hardisson, J. A. Fresno Vara, C. Belda-Iniesta, M. Gonzalez-Baron and J. M. Cuezva, Carcinog., 2005, 26, 2095-2104.

77. T. Koike, N. Kimura, K. Miyazaki, T. Yabuta, K. Kumamoto, S. Takenoshita, J. Chen, M. Kobayashi, M. Hosokawa, A. Taniguchi, T. Kojima, N. Ishida, M. Kawakita, H. Yamamoto, H. Takematsu, A. Suzuki, Y. Kozutsumi and R. Kanangi, PNAS, 2004, 101, 8132-8137.

78. A. Minchenko, I. Leshchinsky, I. Opentanova, N. L. Sang, V. Srinivas, V. Armstead and J. Caro, J. Biol. Chem., 2002, 277, 6183-6187.

79. S. Pavlides, D. Whitaker-Menezes, R. Castello-Cros, N. Flomenberg, A. K. Witkiewicz, P. G. Frank, M. C. Casimiro, C. G. Wang, P. Fortina, S. Addya, R. G. Pestell, U. E. MartinezOutschoorn, F. Sotgia and M. P. Lisanti, Cell Cycle, 2009, 8, 3984-4001.

80. P. L. Pedersen, J. Bioenerge. Biomemb., 2007, 39, 211-222.

81. J. van Reeuwijk, M. Janssen, C. van den Elzen, D. B. V. de Bernabe, P. Sabatelli, L. Merlini, M. Boon, H. Scheffer, M. Brockington, F. Muntoni, M. A. Huynen, A. Verrips, C. A. Walsh, P. G. Barth, H. G. Brunner and H. van Bokhoven, J. Med. Genet., 2005, 42, 907-912.

82. D. C. Wallace and P. Cold Spring Harbor Lab, in Molecular Approaches to Controlling Cancer, 2005, 70, 363-374.

83. P. S. Ward and C. B. Thompson, Cancer Cell, 2012, 21, 297-308.

84. O. Feron, Radiother. Oncol., 2009, 92, 329-333.

85. V. Ganapathy, M. Thangaraju and P. D. Prasad, Pharmacol. Ther., 2009, 121, 29-40.

86. T. J. Schulz, R. Thierbach, A. Voigt, G. Drewes, B. Mietzner, P. Steinberg, A. F. H. Pfeiffer and M. Ristow, J. Biol. Chem., 2006, 281, 977-981. 
87. V. J. Adlam, J. C. Harrison, C. M. Porteous, A. M. James, R. A. J. Smith, M. P. Murphy and I. A. Sammut, Faseb J., 2005, 19, 1088-1095.

88. M. G. Douglas, M. T. McCammon and A. Vassarotti, Microbiol. Rev., 1986, 50, 166-178.

89. S. Fulda, L. Galluzzi and G. Kroemer, Nat. Rev. Drug Discov., 2010, 9, 447-464.

90. J. L. Hickey, R. A. Ruhayel, P. J. Barnard, M. V. Baker, S. J. Berners-Price and A. Filipovska, J. Am. Chem. Soc., 2008, 130, 12570.

91. S. S. Liu, Biosci. Rep., 1997, 17, 259-272.

92. D. C. Logan and C. J. Leaver, J. Exp. Bot., 2000, 51, 865-871.

93. M. Lutter, M. Fang, X. Luo, M. Nishijima, X. S. Xie and X. D. Wang, Nat. Cell Biol., 2000, 2, 754-756.

94. A. M. James, H. M. Cocheme, R. A. J. Smith and M. P. Murphy, J. Biol. Chem., 2005, 280, 21295-21312.

95. M. L. Jauslin, T. Meier, R. A. J. Smith and M. P. Murphy, Faseb J., 2003, 17, 1972.

96. M. P. Murphy and R. A. J. Smith, Annu. Rev. Pharmacol. Toxicol., 2007, 47, 629-656.

97. A. Szewczyk and L. Wojtczak, Pharmacol. Rev., 2002, 54, 101-127.

98. B. Westermann and W. Neupert, Yeast, 2000, 16, 1421-1427.

99. F. Zhou, S. Wu, Y. Yuan, W. R. Chen and D. Xing, Small, 2012, 8, 1543-1550.

100. A. T. Hoye, J. E. Davoren, P. Wipf, M. P. Fink and V. E. Kagan, Acc. Chem. Res., 2008, 41, 8797.

101. W. Neupert, Annu. Rev. Biochem., 1997, 66, 863-917.

102. G. Duby and M. Boutry, Plant Sci., 2002, 162, 477-490.

103. J. Brix, K. Dietmeier and N. Pfanner, J. Biol. Chem., 1997, 272, 20730-20735.

104. K. Zhao, G.-M. Zhao, D. Wu, Y. Soong, A. V. Birk, P. W. Schiller and H. H. Szeto, J. Biol. Chem., 2004, 279, 34682-34690.

105. A. Dhanasekaran, S. Kotamraju, C. Karunakaran, S. V. Kalivendi, S. Thomas, J. Joseph and B. Kalyanaraman, Free Radical Biol. Med., 2005, 39, 567-583.

106. A. M. James, M. S. Sharpley, A.-R. B. Manas, F. E. Frerman, J. Hirst, R. A. J. Smith and M. P. Murphy, J. Biol. Chem., 2007, 282, 14708-14718.

107. Y. J. Hou, S. C. Li, M. Wu, J. Y. Wei, Y. Z. Ren, C. Y. Du, H. J. Wu, C. L. Han, H. J. Duan and Y. H. Shi, Am. J. Physiol.-Renal Physiol., 2016, 310, F547-F559.

108. S. Y. Hao, J. J. Ji, H. T. Zhao, L. C. Shang, J. Wu, H. H. Li, T. Qiao and K. Y. Li, Molecules, 2015, 20, 21287-21297.

109. M. R. de Oliveira, R. F. da Rocha and J. C. F. Moreira, Acta Neuropsychiatrica, 2012, 24, 101108.

110. K. Zhao, G. Luo, S. Giannelli and H. H. Szeto, Biochem. Pharmacol., 2005, 70, 1796-1806.

111. A. M. Byrne, J. J. Lemasters and A.-L. Nieminen, Hepatology, 1999, 29, 1523-1531.

112. S. Ban, H. Nakagawa, T. Suzuki and N. Miyata, Bioorg. Med. Chem. Lett., 2007, 17, 1451-1454.

113. J. Jiang, I. Kurnikov, N. A. Belikova, J. Xiao, Q. Zhao, A. A. Amoscato, R. Braslau, A. Studer, M. P. Fink, J. S. Greenberger, P. Wipf and V. E. Kagan, J. Pharmacol. Exp. Ther., 2007, 320, 10501060 .

114. S. S. Sheu, D. Nauduri and M. W. Anders, Biochimica Et Biophysica Acta-Molecular Basis of Disease, 2006, 1762, 256-265.

115. W. P. Li, P. S. Liu, B. K. Pilcher and R. G. W. Anderson, J. Cell Sci.,, 2001, 114, 1397-1408.

116. F. S. Wu, Planta, 1987, 171, 346-357.

117. J. S. Wolfson, G. L. McHugh, M. N. Swartz, E. Y. W. Ng and D. C. Hooper, J. Parasitol., 1987, 73, 866-869.

118. G. L. Vannini, S. Pancaldi, F. Poli and M. P. Fasulo, Plant Cell Environ., 1988, 11, 123-127.

119. M. L. Thakur, R. Rowley, S. McKenney, S. Cox, D. Leeper and C. Park, J. Labelled Compd. Radiopharm., 1984, 21, 1120-1122.

120. S. Rosenberg, K. K. Nadakavukaren, T. J. Lampidis, S. D. Bernal, I. C. Summerhayes, E. L. Shepherd and L. B. Chen, J. Cell Biol., 1982, 95, A276-A276.

121. K. Ogawa, M. Tsuji, S. Tsuyama and F. Sasaki, Acta Histochemica Et Cytochemica, 2003, 36, 255-262.

122. J. E. Oconnor, J. L. Vargas, B. F. Kimler, J. Hernandezyago and S. Grisolia, Biochem. Biophysi. Res. Commun., 1988, 151, 568-573.

123. J. S. Modicanapolitano, M. J. Weiss, L. B. Chen and J. R. Aprille, Biochem. Biophysi. Res. Commun., 1984, 118, 717-723. 
124. Y. P. Hu, C. T. Moraes, N. Savaraj, W. Priebe and T. J. Lampidis, Biochem. Pharmacol., 2000, 60, 1897-1905.

125. Y. Y. Tyurina, M. A. Tungekar, M. Y. Jung, V. A. Tyurin, J. S. Greenberger, D. A. Stoyanovsky and V. E. Kagan, Febs Lett., 2012, 586, 235-241.

126. C. M. Paleos, D. Tsiourvas and Z. Sideratou, Mol. Pharm., 2016, 13, 2233-2241.

127. T. Murase, T. Yoshihara and S. Tobita, Chem. Lett., 2012, 41, 262-263.

128. D. Y. Cho, H. Cho, K. Kwon, M. Yu, E. Lee, K. M. Huh, D. H. Lee and H. C. Kang, Adv. Func. Mater., 2015, 25, 5479-5491.

129. A. Chakraborty and N. R. Jana, J. Physi. Chem. C, 2015, 119, 2888-2895.

130. S. E. Brown, M. F. Ross, A. Sanjuan-Pla, A. R. B. Manas, R. A. J. Smith and M. P. Murphy, Free Radical Biol. Med., 2007, 42, 1766-1780.

131. M. P. Murphy and R. A. J. Smith, Annual Rev. Pharmacol. Toxicol., 2007, 47, 629-656.

132. C. M. Porteous, A. Logan, C. Evans, E. C. Ledgerwood, D. K. Menon, F. Aigbirhio, R. A. J. Smith and M. P. Murphy, Biochimica Et Biophysica Acta-General Subjects, 2010, 1800, 10091017.

133. I. I. Slowing, C.-W. Wu, J. L. Vivero-Escoto and V. S. Y. Lin, Small, 2009, 5, 57-62.

134. I. I. Slowing, J. L. Vivero-Escoto, C.-W. Wu and V. S. Y. Lin, Adv. Drug Delivery Rev., 2008, 60, 1278-1288.

135. I. I. Slowing, J. L. Vivero-Escoto, B. G. Trewyn and V. S. Y. Lin, J. Mater. Chem., 2010, 20, 7924-7937.

136. I. I. Slowing, B. G. Trewyn and V. S. Y. Lin, J. Am. Chem. Soc., 2007, 129, 8845-8849.

137. M. Martínez-Carmona, M. Colilla and M. Vallet-Regí, Nanomaterials, 2015, 5, 1906.

138. F. Torney, B. G. Trewyn, V. S. Y. Lin and K. Wang, Nat. Nanotech., 2007, 2, 295-300.

139. J. Lu, M. Liong, J. I. Zink and F. Tamanoi, Small, 2007, 3, 1341-1346.

140. J. Lu, M. Liong, Z. Li, J. I. Zink and F. Tamanoi, Small, 2010, 6, 1794-1805.

141. W. F. Yan, B. Chen, S. M. Mahurin, E. W. Hagaman, S. Dai and S. H. Overbury, J. Phys.l Chem. $B, 2004,108,2793-2796$.

142. F. Tang, L. Li and D. Chen, Adv. Mater., 2012, 24, 1504-1534.

143. R. M. Rioux, H. Song, J. D. Hoefelmeyer, P. Yang and G. A. Somorjai, J. Physi. Chem. B, 2005, 109, 2192-2202.

144. S. H. Wu, C. Y. Mou and H. P. Lin, Chem. Soc. Rev., 2013, 42, 3862-3875.

145. P. Yang, S. Gai and J. Lin, Chem. Soc. Rev。, 2012, 41, 3679-3698.

146. Q. He, J. Zhang, J. Shi, Z. Zhu, L. Zhang, W. Bu, L. Guo and Y. chen, Biomaterials, 2010, 31, 1085-1092.

147. I. Gorelikov and N. Matsuura, Nano Lett., 2008, 8, 369-373.

148. M. Colilla, B. Gonzalez and M. Vallet-Regi, Biomater. Sci., 2013, 1, 114-134.

149. H. Song, R. M. Rioux, J. D. Hoefelmeyer, R. Komor, K. Niesz, M. Grass, P. D. Yang and G. A. Somorjai, J. Am. Chem. Soc., 2006, 128, 3027-3037.

150. Y. S. Lin, C. P. Tsai, H. Y. Huang, C. T. Kuo, Y. Hung, D. M. Huang, Y. C. Chen and C. Y. Mou, Chem. Mater., 2005, 17, 4570-4573.

151. T.-H. Chung, S.-H. Wu, M. Yao, C.-W. Lu, Y.-S. Lin, Y. Hung, C.-Y. Mou, Y.-C. Chen and D.M. Huang, Biomaterials, 2007, 28, 2959-2966.

152. T. Yu, A. Malugin and H. Ghandehari, ACS Nano, 2011, 5, 5717-5728.

153. P. P. Yang, S. L. Gai and J. Lin, Chem. Soc. Rev, 2012, 41, 3679-3698.

154. T. M. Sun, Y. S. Zhang, B. Pang, D. C. Hyun, M. X. Yang and Y. N. Xia, Angew. Chem. Int. Ed., 2014, 53, 12320-12364.

155. Q. J. He, J. M. Zhang, J. L. Shi, Z. Y. Zhu, L. X. Zhang, W. B. Bu, L. M. Guo and Y. Chen, Biomaterials, 2010, 31, 1085-1092.

156. Q. J. He, J. L. Shi, F. Chen, M. Zhu and L. X. Zhang, Biomaterials, 2010, 31, 3335-3346.

157. F. Chen, H. Hong, Y. Zhang, H. F. Valdovinos, S. X. Shi, G. S. Kwon, C. P. Theuer, T. E. Barnhart and W. B. Cai, ACS Nano, 2013, 7, 9027-9039.

158. V. Biju, Chem. Soc. Rev., 2014, 43, 744-764.

159. B. J. Marquis, S. A. Love, K. L. Braun and C. L. Haynes, Analyst, 2009, 134, 425-439.

160. T. L. Liu, L. L. Li, X. Teng, X. L. Huang, H. Y. Liu, D. Chen, J. Ren, J. Q. He and F. Q. Tang, Biomaterials, 2011, 32, 1657-1668.

161. L. L. Li, F. Q. Tang, H. Y. Liu, T. L. Liu, N. J. Hao, D. Chen, X. Teng and J. Q. He, ACS Nano, 2010, 4, 6874-6882. 
162. H. Jaganathan and B. Godin, Advanced Drug Delivery Reviews, 2012, 64, 1800-1819.

163. A. J. Di Pasqua, K. K. Sharma, Y. L. Shi, B. B. Toms, W. Ouellette, J. C. Dabrowiak and T. Asefa, J. Inorg. Biochem., 2008, 102, 1416-1423.

164. H. X. Wu, G. Liu, S. J. Zhang, J. L. Shi, L. X. Zhang, Y. Chen, F. Chen and H. R. Chen, J. Mater. Chem., 2011, 21, 3037-3045.

165. D. Tarn, C. E. Ashley, M. Xue, E. C. Carnes, J. I. Zink and C. J. Brinker, Acc. Chem. Res., 2013, 46, 792-801.

166. Q. He, Z. Zhang, Y. Gao, J. Shi and Y. Li, Small, 2009, 5, 2722-2729.

167. H. Meng, S. Yang, Z. Li, T. Xia, J. Chen, Z. Ji, H. Zhang, X. Wang, S. Lin, C. Huang, Z. H. Zhou, J. I. Zink and A. E. Nel, ACS Nano, 2011, 5, 4434-4447.

168. M. A. Malvindi, V. Brunetti, G. Vecchio, A. Galeone, R. Cingolani and P. P. Pompa, Nanoscale, 2012, 4, 486-495.

169. Q. T. Meng, W. P. Su, C. He and C. Y. Duan, Talanta, 2012, 97, 456-461.

170. Q. T. Meng, W. P. Su, X. M. Hang, X. Z. Li, C. He and C. Y. Duan, Talanta, 2011, 86, 408-414.

171. X. H. Liu, H. S. Qian, Y. P. Ji, Z. Q. Li, Y. Shao, Y. Hu, G. X. Tong, L. C. Li, W. D. Guo and H. C. Guo, RSC $A d v$., 2012, 2, 12263-12268.

172. S. Gandhi, K. Thandavan, B. J. Kwon, H. J. Woo, S. S. Yi, H. S. Lee, J. H. Jeong, K. Jang and D. S. Shin, RSC Adv., 2014, 4, 5953-5962.

173. R. Prasad, S. Aiyer, D. S. Chauhan, R. Srivastava and K. Selvaraj, Nanoscale, 2016, 8, 4537 4546.

174. R. C. Lv, C. N. Zhong, A. K. Gulzar, F. He, R. Gu, S. L. Gai, S. H. Zhang, G. X. Yang and P. P. Yang, $R S C A d v ., 2016,6,21590-21599$.

175. C.-P. Tsai, C.-Y. Chen, Y. Hung, F.-H. Chang and C.-Y. Mou, Journal of Materials Chemistry, 2009, 19, 5737-5743.

176. J. M. Rosenholm, C. Sahlgren and M. Linden, Nanoscale, 2010, 2, 1870-1883.

177. Z. Luo, K. Cai, Y. Hu, L. Zhao, P. Liu, L. Duan and W. Yang, Angew. Chem. Int. Ed., 2011, 50, 640-643.

178. S.-H. Wu, Y.-S. Lin, Y. Hung, Y.-H. Chou, Y.-H. Hsu, C. Chang and C.-Y. Mou, Chembiochem, 2008, 9, 53-57.

179. J. L. Vivero-Escoto, I. I. Slowing, B. G. Trewyn and V. S. Y. Lin, Small, 2010, 6, 1952-1967.

180. D. Peer, J. M. Karp, S. Hong, O. C. Farokhzad, R. Margalit and R. Langer, Nat. Nano., 2007, 2, 751-760.

181. G. S. Song, Q. A. Wang, Y. Wang, G. Lv, C. Li, R. J. Zou, Z. G. Chen, Z. Y. Qin, K. K. Huo, R. G. Hu and J. Q. Hu, Adv. Func. Mater., 2013, 23, 4281-4292.

182. Q. H. Yang, M. E. Cogswell, H. C. Hamner, A. Carriquiry, L. B. Bailey, C. M. Pfeiffer and R. J. Berry, Am. J. Clin. Nutr., 2010, 91, 64-72.

183. J. Yang, H. T. Chen, I. R. Vlahov, J. X. Cheng and P. S. Low, J. Pharmacol. Exp. Ther., 2007, 321, 462-468.

184. X. Q. Pan, X. Zheng, G. F. Shi, H. Q. Wang, M. Ratnam and R. J. Lee, Blood, 2002, 100, 594602.

185. E. Hertrampf, F. Cortes, J. D. Erickson, M. Cayazzo, W. Freire, L. B. Bailey, C. Howson, G. P. A. Kauwell and C. Pfeiffer, J. Nutr., 2003, 133, 3166-3169.

186. S. F. Choumenkovitch, P. F. Jacques, M. R. Nadeau, P. W. F. Wilson, I. H. Rosenberg and J. Selhub, J. Nutr. 2001, 131, 3277-3280.

187. R. L. Bailey, K. W. Dodd, J. J. Gahche, J. T. Dwyer, M. A. McDowell, E. A. Yetley, C. A. Sempos, V. L. Burt, K. L. Radimer and M. F. Picciano, American J. Clin. Nutr., 2010, 91, 231237.

188. M. Kramer, J. F. Stumbe, H. Turk, S. Krause, A. Komp, L. Delineau, S. Prokhorova, H. Kautz and R. Haag, Angew. Chem. Int. Ed., 2002, 41, 4252-4256.

189. C. H. Lee, S. H. Cheng, I. P. Huang, J. S. Souris, C. S. Yang, C. Y. Mou and L. W. Lo, Angew. Chem. Int. Ed., 2010, 49, 8214-8219.

190. D. M. Lynn, M. M. Amiji and R. Langer, Angew. Chem. Int. Ed., 2001, 40, 1707-1710.

191. T. Hirai, H. Okubo and I. Komasawa, Journal of Physical Chemistry B, 1999, 103, 4228-4230.

192. J. Zong, Y. H. Zhu, X. L. Yang, J. H. Shen and C. Z. Li, Chem. Commun., 2011, 47, 764-766.

193. C.-Y. Lai, B. G. Trewyn, D. M. Jeftinija, K. Jeftinija, S. Xu, S. Jeftinija and V. S. Y. Lin, J. Am. Chem. Soc., 2003, 125, 4451-4459.

194. R. Hernandez, H.-R. Tseng, J. W. Wong, J. F. Stoddart and J. I. Zink, J. Am. Chem. Soc., 2004, 
126, 3370-3371.

195. S. Fulda, L. Galluzzi and G. Kroemer, Nat. Rev. Drug Discov., 2010, 9, 447-464.

196. A. S. Don, Cancer Cell, 2003, 3, 497-509.

197. I. J. Diel, Ann. Oncol., 2008, 19, 2007-2011.

198. E. A. Bey, Proc. Natl Acad. Sci. USA, 2007, 104, 11832-11837.

199. P. Huang, L. Feng, E. A. Oldham, M. J. Keating and W. Plunkett, Nature, 2000, 407, 390-395.

200. K. Maaser, Clin. Cancer Res., 2005, 11, 1751-1756.

201. Z. Chen, H. Zhang, W. Lu and P. Huang, Biochim. Biophys. Acta, 2009, 1787, 553-560.

202. S. Bonnet, Cancer Cell, 2007, 11, 37-51.

203. X. Cao, Clin. Cancer Res., 2008, 14, 1831-1839.

204. B. H. Kang, Cell, 2007, 131, 257-270.

205. R. H. Cichewicz and S. A. Kouzi, Med. Res. Rev., 2004, 24, 90-114.

206. S. Fulda, Cancer Res., 1997, 57, 4956-4964.

207. L. F. Dong, Oncogene, 2008, 27, 4324-4335.

208. J. Neuzil, FASEB J., 2001, 15, 403-415.

209. J. Zhou, W.-Y. Zhao, X. Ma, R.-J. Ju, X.-Y. Li, N. Li, M.-G. Sun, J.-F. Shi, C.-X. Zhang and W.L. Lu, Biomaterials, 2013, 34, 3626-3638.

210. Q. Qu, X. Ma and Y. Zhao, Nanoscale, 2015, 7, 16677-16686.

211. I. I. Slowing, B. G. Trewyn, S. Giri and V. S. Y. Lin, Adv. Func. Mater., 2007, 17, 1225-1236.

212. A. Popat, S. B. Hartono, F. Stahr, J. Liu, S. Z. Qiao and G. Q. Lu, Nanoscale, 2011, 3, 2801-2818.

213. P. Borst, R. Evers, M. Kool and J. Wijnholds, J. Nat. Cancer Inst., 2000, 92, 1295-1302.

214. Y. H. Cui, J. Konig, U. Buchholz, H. Spring, I. Leier and D. Keppler, Mol. Pharmacol., 1999, 55, 929-937.

215. M. Dean, T. Fojo and S. Bates, Nat. Rev. Cancer, 2005, 5, 275-284.

216. M. M. Gottesman, Annu. Rev. Med., 2002, 53, 615-627.

217. R. M. Kaplan, Trends in Parasitol., 2004, 20, 477-481.

218. S. J. Little, S. Holte, J. P. Routy, E. S. Daar, M. Markowitz, A. C. Collier, R. A. Koup, J. W. Mellors, E. Connick, B. Conway, M. Kilby, L. Wang, J. M. Whitcomb, N. S. Hellmann and D. D. Richman, N. Engl. J. Med., 2002, 347, 385-394.

219. D. B. Longley and P. G. Johnston, J. Pathol., 2005, 205, 275-292.

220. A. P. Magiorakos, A. Srinivasan, R. B. Carey, Y. Carmeli, M. E. Falagas, C. G. Giske, S. Harbarth, J. F. Hindler, G. Kahlmeter, B. Olsson-Liljequist, D. L. Paterson, L. B. Rice, J. Stelling, M. J. Struelens, A. Vatopoulos, J. T. Weber and D. L. Monnet, Clin. Microbiol. Infect., 2012, 18, 268-281.

221. H. S. L. Chan, G. Haddad, P. S. Thorner, G. Deboer, Y. P. Lin, N. Ondrusek, H. Yeger and V. Ling, N. Engl. J. Med., 1991, 325, 1608-1614.

222. C. Cordoncardo, J. P. Obrien, D. Casals, L. Rittmangrauer, J. L. Biedler, M. R. Melamed and J. R. Bertino, PNAS, 1989, 86, 695-698.

223. B. Greiner, M. Eichelbaum, P. Fritz, H. P. Kreichgauer, O. Von Richter, J. Zundler and H. K. Kroemer, J. Clin. Invest., 1999, 104, 147-153.

224. J. W. Polli, S. A. Wring, J. E. Humphreys, L. Y. Huang, J. B. Morgan, L. O. Webster and C. S. Serabjit-Singh, J. Pharmacol. Exp. Ther., 2001, 299, 620-628. 


\section{Conclusion and Outcome}

In conclusion, this thesis described my research progress during postgraduate study on fabricating effective MSNPs based mitochondria targeted sub-cellular drug delivery system and its application in anticancer treatment and overcoming drug resistance. The research projects were conducted with background of validations of MSNPs in biological applications such as drug delivery application and bio-imaging application. What's more, the research motivations were establish based on the fact that insufficient investigations could be found in applying MSNPs in sub-cellular application.

In the effort to validate the application in sub-cellular drug delivery, MSNPs with proper particle size were functionalized with mitochondria targeting ligand with aim to target mitochondria. After loading with DOX, mitochondria targeted MSNPs showed better anticancer efficiency compared with plain MSNPs, indicating the good performance of MSNPs in targeting and delivering drug molecules to mitochondria. Beyond that, the mitochondria targeted MSNPs with reduced particle size were further loaded with mitochondria specific hydrophobic drug molecules, $\alpha$-TOS. The hydrophobicity of $\alpha$ TOSwas overcome and the anticancereffectiveness was further maximized. In addition, the application of mitochondria targeted MSNPs was further broadened by applying such MSNPs in overcoming drug resistance. By changing the target site of DOX from nucleus DNAs to mtDNAs and decreasing the ATP supply as well as P-gp activity, the apoptosis process could be effectively induced by DOX delivered by mitochondria targeted MSNPs. Thus, DOX resistance was effectively overcome. Above all, MSNPs were demonstrated to be suitable in sub-cellular drug delivery. 
However, there are lots of future work need to be conducted. For example, in order to implement the anticancer as well as anti-drug resistance applications of MSNPs in subcellular drug delivery require detailed and complete in vivo investigation. In addition, more detailed toxicity study of MSNPs in human body system is of great importance. Herein, the application of MSNPs in sub-cellular drug delivery is definitely at its infancy, and a huge potential of MSNPs in benefiting future drug delivery research has yet to be discovered.

\section{List of Publications}

1. Qiuyu Qu, Xing Ma, Yanli Zhao,* Anticancer Effect of $\alpha$-Tocopheryl Succinate Delivered byMitochondria-Targeted Mesoporous Silica Nanoparticles. ACS Appl. Mater. Interfaces 2016, Accepted.

2. Qiuvu Qu, Xing Ma, Yanli Zhao,* Targeted delivery of doxorubicin to mitochondria using mesoporous silica nanoparticle nanocarriers. Nanoscale2015, 7, 16677-16686.

3. Hongzhong Chen, Huijun Phoebe Tham, Chung Yen Ang, Qiuvu Qu, Lingzhi Zhao, Pengyao Xing, Linyi Bai, Si Yu Tan, Yanli Zhao,* Responsive prodrug self-assembled vesicles for targeted chemotherapy in combination with intracellular imaging.ACS Appl. Mater. Interfaces 2016, 8, 24319-24324.

4. Desiree-Faye KaixinToh, Gitali Devi, Kiran M. Patil, Qiuyu Qu, ManikanthaMaraswami, Yunyun Xiao, Teck Peng Loh, Yanli Zhao, ${ }^{*}$ Gang Chen, ${ }^{*}$ Incorporating a guanidine-modified cytosine base into triplexforming PNAs for the recognition of a C-G pyrimidine-purine inversion site of an RNA duplex. Nucl.Acids Res. 2016.

5. Huijun Phoebe Tham, Hongzhong Chen, Yu Hui Tan, Qiuvu Qu, SivaramapanickerSreejith, Lingzhi Zhao, Subbu S. Venkatraman,* Yanli Zhao,* Photosensitizer anchored gold nanorods for targeted combinational photothermal and photodynamic therapy. Chem. Commun. 2016.

6. Lingzhi Zhao, Wei Yuan, Chung Yen Ang, Qiuyu Qu, Yu Dai, Yilin Gao, Zhong Luo, Jin-Gui Wang, Hongzhong Chen, Menghuan Li, Fuyou Li, ${ }^{*}$ Yanli Zhao, ${ }^{*}$ Silica-polymer hybrid with self-assembled PEG corona excreted rapidly via a hepatobiliary route. Adv. Funct. Mater. 2016, 26, 3036-3047.

7. Menghuan Li, Cathleen Teh, Chung Yen Ang, Si Yu Tan, Zhong Luo, Qiuyu Qu, Yuanyuan Zhang, Vladimir Korzh, Yanli Zhao, ${ }^{*}$ Near-infrared light-absorptive stealth liposomes for localized photothermal ablation of tumors combined with chemotherapy. Adv. Funct. Mater.2015, 25, 5602-5610.

8. Xing Ma, Qiuyu Qu, Yanli Zhao,* Targeted delivery of 5-aminolevulinic acid by multifunctional hollow mesoporous silica nanoparticles for photodynamic skin cancer therapy. ACS Appl. Mater. Interfaces 2015, 7, 10671-10676.

9.Huifang Shi, Xing Ma, Qiang Zhao, Bin Liu, Qiuyu Qu, Zhongfu An, Yanli Zhao*, Wei Huang,* Ultrasmall phosphorescent polymer dots for ratiometric oxygen sensing and photodynamic cancer therapy. Adv. Funct. Mater. 2014,24, 4823-4830. 
10. Xing Ma, Qiuvu Qu, Yun Zhao, Zhong Luo, Yang Zhao, KeeWoei Ng, Yanli Zhao,* Graphene oxide wrapped gold nanoparticles for intracellular Raman imaging and drug delivery. J. Mater. Chem. B 2013, 1, 6495-6500. 